\title{
Partitions and quasimodular forms
}

Variations on the Bloch-Okounkov theorem

\author{
Jan-Willem van Ittersum
}

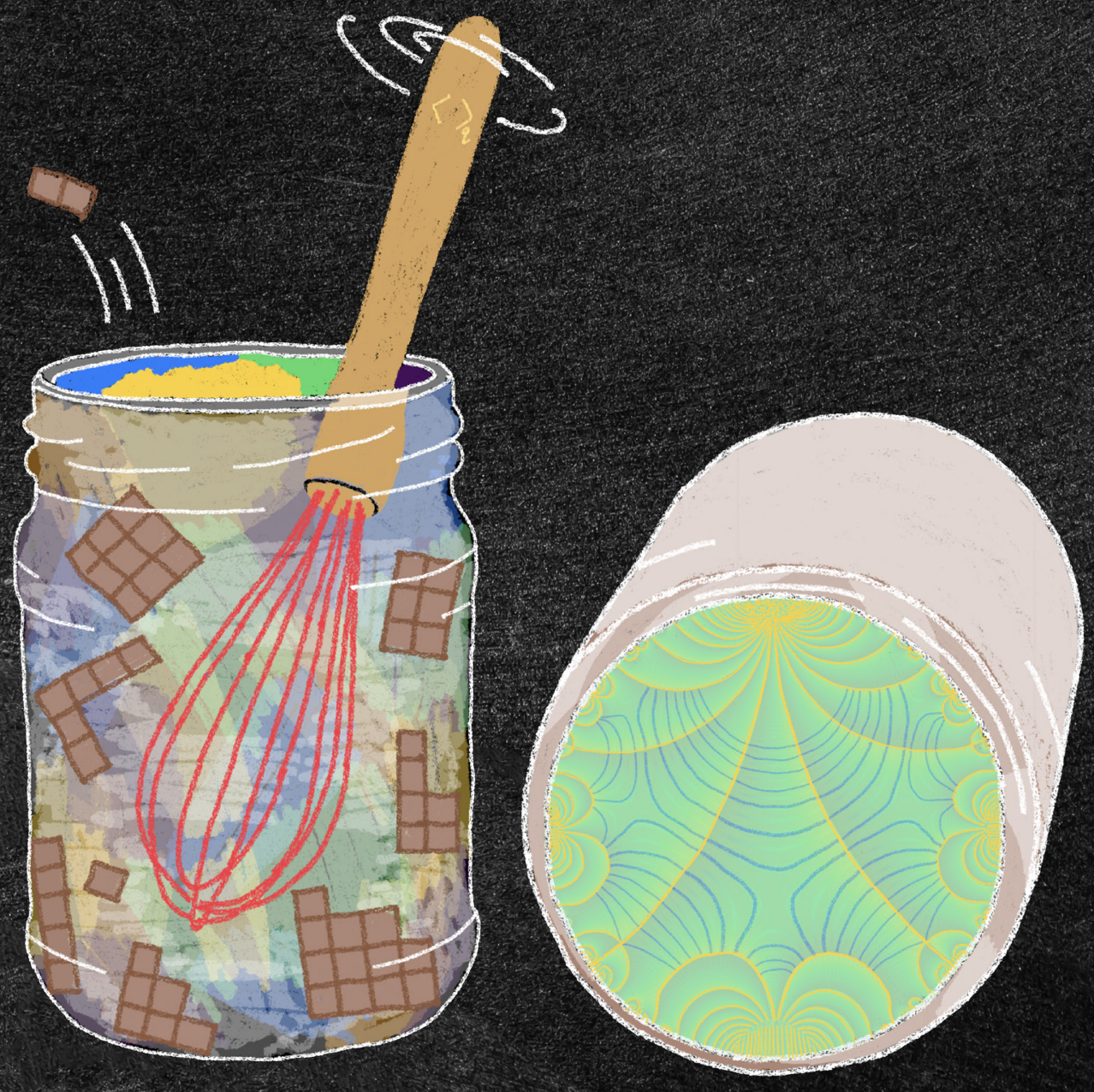


Partitions and quasimodular forms

Variations on the Bloch-Okounkov theorem 


\section{Thesis committee:}

Prof. dr. Frits Beukers, Universiteit Utrecht

Prof. dr. Kathrin Bringmann, Universität zu Köln

Prof. dr. Masanobu Kaneko, 九州大学

Prof. dr. Martin Möller, Goethe-Universität Frankfurt

Prof. dr. Sameer Murthy, King's College London

Zie de Nederlandse samenvatting voor een uitleg van wat er op de omslag van dit proefschrift te zien is.

ISBN: 978-90-393-7378-1

Copyright $\odot 2021$ by Jan-Willem M. van Ittersum. All rights reserved. 


\title{
Partitions and quasimodular forms Variations on the Bloch-Okounkov theorem
}

\author{
Partities en quasimodulaire vormen \\ Variaties op de Bloch-Okounkov stelling \\ (met een samenvatting in het Nederlands)
}

\section{Proefschrift}

ter verkrijging van de graad van doctor aan de Universiteit Utrecht op gezag van de rector magnificus, prof. dr. H. R. B. M. Kummeling, ingevolge het besluit van het college voor promoties in het openbaar te verdedigen op

woensdag 9 juni 2021 des ochtends te 10.15 uur

door

Johannes Wilhelmus Maria van Ittersum

geboren op 10 augustus 1994

te Amstelveen 
Promotoren: Prof. dr. G. L. M. Cornelissen

Prof. dr. D. B. Zagier 
Sol iustitiae illustra nos 

1 Introduction $\quad 1$

1.1 Functions on partitions and the $q$-bracket . . . . . . . . . . . . . 1

1.2 The $\mathfrak{s l}_{2}$-algebra of quasimodular forms ............ 3

1.3 Shifted symmetric functions and hook-length moments . . . . . . 7

1.4 The Jacobi theta series and the Bloch-Okounov theorem . . . . . . 10

1.5 Motivation from enumerative geometry ... . . . . . . . 11

1.6 Main questions and results ............... 14

I Results on the Bloch-Okounkov algebra 21

2 When is the Bloch-Okounkov $q$-bracket modular? 23

2.1 Introduction . . . . . . . . . . . . . . . 23

2.2 An $\mathfrak{s l}_{2}$-equivariant Bloch-Okounkov theorem . . . . . . . . . 25

2.3 Describing the space of shifted symmetric harmonic polynomials . . 29

3 Quasimodularity of Hurwitz numbers over elliptic curves 35

3.1 Introduction . . . . . . . . . . . . . . . 35

3.2 Triply mixed Hurwitz numbers $\ldots \ldots \ldots \ldots$

3.3 Central characters of the symmetric group are shifted symmetric . . 38

3.4 Quasimodularity of triply mixed Hurwitz numbers . . . . . . . . 40

II A symmetric Bloch-Okounkov theorem 45

4 New quasimodular algebras $\quad 47$

4.1 Introduction . . . . . . . . . . . . . . . . . 47

4.2 Preliminaries ....................... 50

4.3 Three proofs of the quasimodularity of the moment functions . . . 54 
4.4 The induced and connected product . . . . . . . . . . 58

4.5 Quasimodularity of pointwise products of moment functions . . . . 61

4.6 Intermezzo: surjectivity of the $q$-bracket $\ldots \ldots \ldots$. . . . . 63

4.7 The connected product of moment functions . . . . . . . . . . 64

4.8 Three quasimodular algebras $\ldots \ldots \ldots \ldots \ldots . \ldots \ldots 72$

4.9 The derivative of a function on partitions . . . . . . . . . . . 77

4.10 The equivariant $q$-bracket and Rankin-Cohen brackets . . . . . . 79

4.11 The structure constants: from the pointwise to the induced product . 82

4.12 Border strip moments and moments of other partition invariants . . 87

III Quasi-Jacobi forms and applications 95

5 Taylor coefficients of quasi-Jacobi forms 97

5.1 Introduction . . . . . . . . . . . . . . . . . . . . . . 97

5.2 Strictly meromorphic Jacobi forms . . . . . . . . . . . . . . . . 99

5.3 The action of the Jacobi group . . . . . . . . . . . . . . . 103

5.4 Poles of Jacobi forms . . . . . . . . . . . . . . . . . . . . . . . . 107

5.5 An approximation lemma . . . . . . . . . . . . . . . . 110

5.6 Quasi-Jacobi forms . . . . . . . . . . . . . . . . . . . . . . . 114

5.7 Action of the Jacobi Lie algebra by derivations . . . . . . . . . 117

5.8 The double slash operator . . . . . . . . . . . . . . . . . 118

5.9 Taylor coefficients . . . . . . . . . . . . . . . . . . . . . . 121

6 The Bloch-Okounkov theorem for congruence subgroups 131

6.1 Introduction . . . . . . . . . . . . . . . . . 131

6.2 General set-up . . . . . . . . . . . . . . . . . . . 133

6.3 First application: the Bloch-Okounkov theorem of higher level . . . 137

6.4 Second application: hook-length moments of higher level . . . . . 138

6.5 Third application: moment functions of higher level . . . . . . . . 139

6.6 Fourth application: double moment functions of higher level . . . . 140

7 When is the $q$-bracket modular? 143

7.1 Introduction . . . . . . . . . . . . . . . . . . 143

7.2 Construction of functions with modular $q$-bracket . . . . . . . . 144

7.3 First example: The Bloch-Okounkov algebra . . . . . . . . . . 146

7.4 Second example: the Bloch-Okounkov algebra of higher level . . 150

7.5 Third example: double moment functions . . . . . . . . . . . 151 
8 A Kaneko-Zagier equation for quasi-Jacobi forms 153

8.1 Introduction . . . . . . . . . . . . . . . . . 153

8.2 The solutions of the differential equation . . . . . . . . . 158

8.3 Three expansions of the solutions . . . . . . . . . . . . . 161

8.4 Holomorphic anomaly equations . . . . . . . . . . . . . . 163

8.5 A second differential equation . . . . . . . . . . . . 166

8.6 Holomorphic anomaly equations II . . . . . . . . . . . . . . . 173

8.7 The classical Kaneko-Zagier equation . . . . . . . . . . . . . 175

$\begin{array}{ll}\text { Appendix } & 179\end{array}$

A Tables of functions on partitions and quasimodular forms 179

A.1 Shifted symmetric harmonic polynomials up to weight $10 \ldots \ldots$. . 179

A.2 Examples of quasimodular generating series of Hurwitz numbers . . 180

A.3 Double moment functions up to weight $4 \ldots \ldots \ldots 181$

$\begin{array}{ll}\text { Samenvatting } & 183\end{array}$

$\begin{array}{ll}\text { Dankwoord } & 189\end{array}$

$\begin{array}{ll}\text { Curriculum Vitae } & 191\end{array}$

$\begin{array}{ll}\text { Bibliography } & 193\end{array}$ 

CHAPTER 1

\section{Introduction}

\subsection{Functions on partitions and the $q$-bracket}

A partition of an integer $n$ is way of writing $n$ as a sum of positive integers, where the order does not matter. For example, the partitions of 5 are given by

$$
5,4+1,3+2,3+1+1,2+2+1,2+1+1+1,1+1+1+1+1,
$$

which one can depict graphically by their Young diagrams as
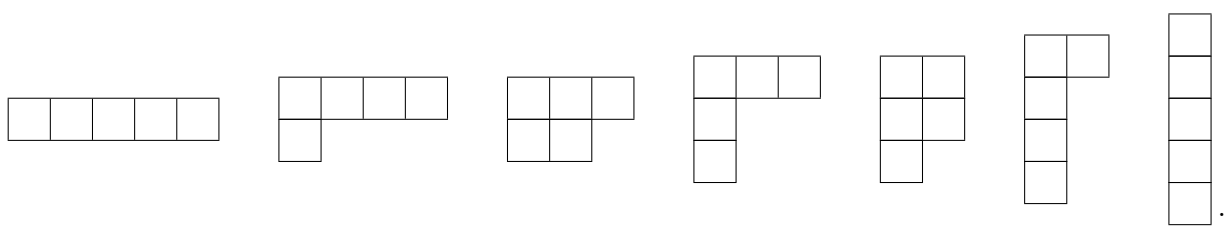

For each integer $n$ the partition function $p(n)$ gives the number of partitions of $n$.

\begin{tabular}{c|c|c|c|c|c|c|c|c|c|c|c|c|c|c|c}
$n$ & 0 & 1 & 2 & 3 & 4 & 5 & 6 & 7 & 8 & 9 & 10 & 11 & 12 & 13 & 14 \\
\hline$p(n)$ & 1 & 1 & 2 & 3 & 5 & 7 & 11 & 15 & 22 & 30 & 42 & 56 & 77 & 101 & 135
\end{tabular}

Table 1.1: The initial values of the partition function.

Its generating series, often the best way of studying a sequence of integers, goes back to Euler who proved that

$$
\sum_{n=0}^{\infty} p(n) q^{n}=\prod_{m=1}^{\infty} \frac{1}{1-q^{m}}
$$

as a formal power series in $q$. Let $\tau \in \mathbb{C}$ with $\operatorname{Im}(\tau)>0$. Write $q=e^{2 \pi \mathrm{i} \tau}$. Then, the right-hand side of this equation is a holomorphic function of $\tau$ that equals $q^{1 / 24} \eta(\tau)^{-1}$, where the Dedekind eta function $\eta$ is given by

$$
\eta(\tau)=q^{1 / 24} \prod_{m=1}^{\infty}\left(1-q^{m}\right) .
$$


This function is a holomorphic modular form (of half-integral weight), in particular, it satisfies the functional equations

$$
\eta(\tau+1)=e^{\frac{2 \pi \mathrm{i}}{24}} \eta(\tau), \quad \eta\left(-\frac{1}{\tau}\right)=\sqrt{-\mathrm{i} \tau} \eta(\tau) .
$$

In this thesis we will be concerned with generalisations of Euler's result. Namely, instead of the generating series (1.1), we consider a weighted count of all partitions. By this we mean the following. Let $\mathscr{P}$ be the set of all integer partitions and for $\lambda \in \mathscr{P}$, write $|\lambda|$ for the integer where $\lambda$ is a partition of. The $q$-bracket, which is the analogue of the expectation value of an observable in statistical mechanics, is the weighted average value of a function $f$ on $\mathscr{P}$, where the weight of $\lambda \in \mathscr{P}$ is $q^{|\lambda|}$ for some formal parameter $q$ :

$$
\langle f\rangle_{q}:=\frac{\sum_{\lambda \in \mathscr{P}} f(\lambda) q^{|\lambda|}}{\sum_{\lambda \in \mathscr{P}} q^{|\lambda|}} \in \mathbb{C}[[q]] .
$$

Note that the denominator of the $q$-bracket equals Euler's generating series (1.1).

We are particularly interested in functions $f$ for which $\langle f\rangle_{q}$ is not only a formal power series in $\mathbb{C}[[q]]$, but also a holomorphic function of $\tau$, where again $q=e^{2 \pi \mathrm{i} \tau}$, with algebraic properties such as the functional equations satisfied by the $\eta$-function. For example, let $f(\lambda)=|\lambda|$. Then

$$
\langle f\rangle_{q}=q \frac{\partial}{\partial q} \log \left(\sum_{\lambda \in \mathscr{P}} q^{|\lambda|}\right)=\frac{1}{24}+G_{2},
$$

where $G_{2}$ is a pivotal player in this thesis: the quasimodular Eisenstein series given in Equation (1.7) below. Stated very naively, the main question of this thesis is as follows.

Question 1.1.1. For which functions $f: \mathscr{P} \rightarrow \mathbb{C}$ is $\langle f\rangle_{q}$ a nice function with good algebraic properties?

A surprising answer to this question is given by Bloch and Okounkov [BO00]. Let $\Lambda^{*}=\mathbb{Q}\left[Q_{2}, Q_{3}, \ldots\right]$ be the graded algebra of shifted symmetric functions (to be introduced in Section 1.3), containing for example the function $Q_{2}(\lambda)=|\lambda|-\frac{1}{24}$ of weight 2 and a generator $Q_{k}$ of weight $k$ for every integer $k \geq 2$.

Theorem 1.1.2 (Bloch-Okounkov theorem). For all $f \in \Lambda^{*}$, the $q$-bracket of $f$ is a quasimodular form of the same weight as $f$.

We now first introduce quasimodular forms and the Bloch-Okounkov algebra $\Lambda^{*}$ of shifted symmetric functions, which will be the leading example through this thesis. Then, after motivating everything from the viewpoint of enumerative geometry, we will refine Question 1.1.1. 


\subsection{The $\mathfrak{s l}_{2}$-algebra of quasimodular forms}

There is a saying attributed to Eichler saying "There are five elementary arithmetical operations: addition, subtraction, multiplication, division, and... modular forms." The goal of this section is to show that all these five operations behave well with respect to yet another operation: derivations.

Eisenstein's method for constructing modular forms carries over to the simpler case of trigonometric functions, which we explain first. Namely, in order to construct a periodic function, consider

$$
\epsilon_{k}(x)=\sum_{n \in \mathbb{Z}}(x+n)^{-k} \quad(k \geq 1) .
$$

This series converges absolutely for $k \geq 2$ and for $k=1$ we apply Eisenstein summation, i.e., we replace the sum by $\lim _{N \rightarrow \infty} \sum_{n=-N}^{N}$. In terms of well-known trigonometric functions, the first three are given by

$$
\epsilon_{1}(x)=\pi \frac{\cos (\pi x)}{\sin (\pi x)}, \quad \epsilon_{2}(x)=\frac{\pi^{2}}{\sin ^{2}(\pi x)}, \quad \epsilon_{3}(x)=\pi^{3} \frac{\cos (\pi x)}{\sin ^{3}(\pi x)} .
$$

Let $T$ be the $\mathbb{Q}$-algebra generated by these functions, i.e., $T=\mathbb{Q}\left[\epsilon_{1}, \epsilon_{2}, \ldots\right]$. All $f \in T$ are periodic with period 1 and all non-constant $f \in T$ admit a pole at all integer values of $x$. The algebra $T$ is an example of the kind of algebras encountered in this thesis, that is, we show that the algebra $T$ (i) is a graded algebra, (ii) is finitely and freely generated and (iii) is closed under differentiation.

To start with the latter, note that for all $k \geq 1$

$$
\frac{\partial}{\partial x} \epsilon_{k}(x)=-k \epsilon_{k+1}(x) \text {. }
$$

Next, one observes that

$$
\epsilon_{3}(x)=\epsilon_{1}(x) \epsilon_{2}(x),
$$

from which, after an $(k-3)$-fold application of (1.3), it follows that

$$
(k-1) \epsilon_{k}(x)=\sum_{i=1}^{k-1} \epsilon_{i}(x) \epsilon_{k-i}(x)
$$

for $k \geq 3$. Indeed, $T$ is finitely generated, namely $T=\mathbb{Q}\left[\epsilon_{1}, \epsilon_{2}\right]$. Finally, note that the analogue of (1.4) for $k=2$ is given by

$$
\epsilon_{2}(x)=\epsilon_{1}(x)^{2}+\pi^{2} .
$$

Hence, $T=\mathbb{Q}\left[\pi^{2}, \epsilon_{1}\right]$, from which it is clear that $T$ is freely generated. 
Assign to $\epsilon_{k}$ weight $k$. By (1.4) and (1.5) this indeed defines a weight grading on $T$, for which the weight of $\pi^{2}$ is 2 . We conclude that $T$ is a finitely generated graded differential algebra. In particular, given a weight $k$, the vector space $T_{k}$ of elements in $T$ of weight $k$ is finite dimensional. This property-which reduces the question whether a given function is an element of $T_{k}$, to comparing finitely many coefficients in the Fourier expansion-is an essential property of the "nice functions with good algebraic properties" in Question 1.1.1.

Now, we are ready to introduce the algebra of quasimodular forms, satisfying this property as well as the aforementioned three properties of the algebra $T$. Let $\mathfrak{H}$ be the upper half plane, i.e., the set of complex numbers $\tau$ for which $\operatorname{Im}(\tau)>0$. The group $\mathrm{SL}_{2}(\mathbb{Z})$ acts on $\mathfrak{H}$ by Möbius transformations. Given $\gamma=\left(\begin{array}{ll}a & b \\ c & d\end{array}\right) \in \mathrm{SL}_{2}(\mathbb{Z})$, $\tau \in \mathfrak{H}$ and $k \in \mathbb{Z}$, we let $\gamma \tau=\frac{a \tau+b}{c \tau+d}$ and define the slash operator in weight $k$ by

$$
\left(\left.f\right|_{k} \gamma\right)(\tau):=(c \tau+d)^{-k} f(\gamma \tau) .
$$

Let $\operatorname{Hol}_{0}(\mathfrak{h})$ be the ring of holomorphic functions $\varphi$ of moderate growth on $\mathfrak{H}$, i.e., for all $C>0, \gamma \in \mathrm{SL}_{2}(\mathbb{Z})$ and $x \in \mathbb{R}$ one has $\left(\left.\varphi\right|_{0} \gamma\right)(x+\mathrm{i} y)=O\left(e^{C y}\right)$ as $y \rightarrow \infty$.

In order to obtain modular forms- $f \in \operatorname{Hol}_{0}(\mathfrak{h})$ for which $\left.f\right|_{k} \gamma=f$ for all $\gamma \in \mathrm{SL}_{2}(\mathbb{Z})$-Eisenstein defined

$$
e_{k}(\tau)=\sum_{\substack{\omega \in \mathbb{Z} \tau+\mathbb{Z} \\ \omega \neq 0}} \frac{1}{\omega^{k}} \quad(k \geq 3) .
$$

The Eisenstein series $e_{k}$ are non-zero modular form of weight $k$ whenever $k$ is even. In case $k=2$, we write $\omega=m \tau+n$ and we define $e_{2}$ by replacing the sum over $\omega$ by $\sum_{m} \sum_{n}$, where $(m, n) \neq(0,0)$. Then,

$$
\left(\left.e_{2}\right|_{2} \gamma\right)(\tau)=e_{2}-2 \pi \mathrm{i} \frac{c}{c \tau+d} .
$$

Hence, $e_{2}$ is not a modular form. Note that both $e_{2}$ and the trigonometric function $\epsilon_{1}$ are periodic functions corresponding to a series which is not absolutely convergent. We proceed by defining quasimodular forms, which are functions satisfying a more general modular transformation property than the Eisenstein series and of which $e_{2}$ is the first non-modular example. Let $\Gamma$ be a subgroup of $\mathrm{SL}_{2}(\mathbb{Z})$ of finite index.

Definition 1.2.1. A quasimodular form of weight $k$ and depth at most $p$ for $\Gamma$ is a function $\varphi \in \operatorname{Hol}_{0}(\mathfrak{H})$ such that there exist $\varphi_{0}, \ldots, \varphi_{p} \in \operatorname{Hol}_{0}(\mathfrak{H})$ so that for all $\tau \in \mathfrak{H}$ and all $\gamma=\left(\begin{array}{ll}a & b \\ c & d\end{array}\right) \in \Gamma$ one has

$$
\left(\left.\varphi\right|_{k} \gamma\right)(\tau)=\varphi_{0}(\tau)+\varphi_{1}(\tau) \frac{c}{c \tau+d}+\ldots+\varphi_{p}(\tau)\left(\frac{c}{c \tau+d}\right)^{p} .
$$


Remark 1.2.2. Every quasimodular form has a Fourier expansion

$$
\varphi(\tau)=\sum_{n \geq 0} a_{n} q_{h}^{n} \quad\left(q_{h}=e^{2 \pi \mathrm{i} \tau / h}\right)
$$

with Fourier coefficients $a_{n} \in \mathbb{C}$ and $h \in \mathbb{Z}_{\geq 1}$ minimal such that $\left(\begin{array}{ll}1 & h \\ 0 & 1\end{array}\right) \in \Gamma$.

Definition 1.2.3. Write $\widetilde{M}_{k}^{(\leq p)}(\Gamma)$ for the vector space of quasimodular forms of weight $k$ and depth at most $p$ for $\Gamma$ with Fourier coefficients $a_{n} \in \mathbb{Q}\left(\zeta_{N}\right)$ (with $N$ the level of $\Gamma$ and $\left.\zeta_{N}=e^{2 \pi \mathrm{i} / N}\right)$ and denote

$$
\widetilde{M}(\Gamma)=\bigoplus_{k} \widetilde{M}_{k}(\Gamma), \quad \widetilde{M}_{k}(\Gamma)=\bigcup_{p} \widetilde{M}_{k}^{(\leq p)}(\Gamma) .
$$

An element of $\widetilde{M}(\Gamma)$ is called a quasimodular form of mixed weight (as it is a linear combination of quasimodular forms of different weight). Analogously, we use $M(\Gamma)$ to denote the space of modular forms for $\Gamma$.

Equation (1.6) is called the quasimodular transformation property. Note that if $\varphi$ is a quasimodular form, the functions $\varphi_{0}, \ldots, \varphi_{p}$ are quasimodular forms uniquely determined by $\varphi$ (the function $\varphi_{r}$ has weight $k-2 r$ and depth $\leq p-r$ ) [Zag08]. For example, taking $\gamma$ in (1.6) equal to the identity $I \in \Gamma$ yields $\varphi_{0}=\varphi$. Quasimodular forms of depth 0 are modular forms. Besides the constant functions, the simplest examples are the Eisenstein series $e_{k}$ for positive even integers $k$. Often, we make use of a slightly different normalisation:

$$
G_{k}(\tau):=\frac{(k-1) !}{2(2 \pi \mathrm{i})^{k}} e_{k}=-\frac{B_{k}}{2 k}+\sum_{r=1}^{\infty} \sum_{m=1}^{\infty} m^{k-1} q^{m r},
$$

where $B_{k}$ is the $k$ th Bernoulli number and $q=e^{2 \pi \mathrm{i} \tau}$. The Eisenstein series $G_{2}$ is a quasimodular form of weight 2 and depth 1.

The differential operator

$$
D_{\tau}=\frac{1}{2 \pi \mathrm{i}} \frac{\mathrm{d}}{\mathrm{d} \tau}=q \frac{\mathrm{d}}{\mathrm{d} q},
$$

which we often abbreviate by $D$, preserves the space of quasimodular forms. In fact, $D: \widetilde{M}_{k}^{(\leq p)}(\Gamma) \rightarrow \widetilde{M}_{k+2}^{(\leq p+1)}(\Gamma)$. Besides $D$, an important differential operator on quasimodular forms is the operator $\mathfrak{d}: \widetilde{M}_{k}^{(\leq p)}(\Gamma) \rightarrow \widetilde{M}_{k-2}^{(\leq p-1)}(\Gamma)$ defined by $\varphi \mapsto 2 \pi \mathrm{i} \varphi_{1}$ (with $\varphi_{1}$ defined by the quasimodular transformation property (1.6)). For example $\mathfrak{d} G_{2}=-\frac{1}{2}$ and in fact this property together with the fact that $\mathfrak{d}$ annihilates modular forms defines $\mathfrak{d}$ completely since $\mathfrak{d}$ is a derivation 
and $\widetilde{M}(\Gamma)=M(\Gamma)\left[G_{2}\right]$ (see [Zag08, Proposition 20] for this equality, which holds for any congruence group $\Gamma$ ).

Let $W$ be the weight operator, which multiplies a quasimodular form by its weight. The triple $(D, W, \mathfrak{d})$ forms an $\mathfrak{s l}_{2}$-triple with respect to the commutator bracket $[A, B]=A B-B A$ :

Definition 1.2.4. A triple $(X, H, Y)$ of operators is called an $\mathfrak{s l}_{2}$-triple if

$$
[H, X]=2 X, \quad[H, Y]=-2 Y, \quad[Y, X]=H .
$$

In case the operators act on an algebra $A$ by derivations, we call the algebra $A$, together with the triple $(X, H, Y)$, an $\mathfrak{s l}_{2}$-algebra.

As $D, W$ and $\mathfrak{d}$ are derivations, the algebra $\widetilde{M}(\Gamma)$ becomes an $\mathfrak{s l}_{2}$-algebra. This is a special case of the more general notion of $\mathfrak{g}$-algebra for any Lie algebra $\mathfrak{g}$ that will be mentioned in Section 5.7, namely, an algebra $A$ together with a Lie algebra homomorphism from $\mathfrak{g}$ to the space $\operatorname{Der}(A)$ of derivations of $A$ with Lie bracket given by commutator.

Example 1.2.5. In case we specialise $\Gamma$ to $\mathrm{SL}_{2}(\mathbb{Z})$, the algebra $M$ of modular forms for $\mathrm{SL}_{2}(\mathbb{Z})$ and the algebra $\widetilde{M}$ of quasimodular forms for $\mathrm{SL}_{2}(\mathbb{Z})$ can be represented as a free polynomial algebra. Recall that by assumption the Fourier coefficients of elements in $M$ are rational. That is, $M=\mathbb{Q}[Q, R]$ and $\widetilde{M}=\mathbb{Q}[P, Q, R]$, where $P=$ $-24 G_{2}, Q=240 G_{4}, R=-504 G_{6}$ are Ramanujan's notation for the Eisenstein series of weight 2,4 and 6, respectively (normalised such that the constant term in the Fourier expansion is 1).

In this representation, the derivations $D$ and $\mathfrak{d}$ are uniquely determined by

$$
D(P)=\frac{P^{2}-Q}{12}, \quad D(Q)=\frac{P Q-R}{3}, \quad D(R)=\frac{P R-Q^{2}}{2},
$$

and

$$
\mathfrak{d}(P)=12, \quad \mathfrak{d}(Q)=0, \quad \mathfrak{d}(R)=0 .
$$

It may happen that a polynomial in the derivatives of two modular forms $f \in$ $M_{k}(\Gamma)$ and $g \in M_{l}(\Gamma)$ is actually modular [Ran56]. This is the case for the RankinCohen brackets of $f$ and $g$ [Coh75], defined by

$$
[f, g]_{n}=\sum_{\substack{r, s \geq 0 \\
r+s=n}}(-1)^{r}\left(\begin{array}{c}
k+n-1 \\
s
\end{array}\right)\left(\begin{array}{c}
l+n-1 \\
r
\end{array}\right) D^{r} f D^{s} g \quad(n \geq 0) .
$$

That is, for $n \geq 0$, the mapping $\widetilde{M}_{k}(\Gamma) \times \widetilde{M}_{l}(\Gamma) \rightarrow \widetilde{M}_{k+l+2 n}(\Gamma)$ given by $(f, g) \mapsto$ $[f, g]_{n}$ restricts to a mapping $M_{k}(\Gamma) \times M_{l}(\Gamma) \rightarrow M_{k+l+2 n}(\Gamma)$. A proof of this statement follows from the fact that $\widetilde{M}$ is an $\mathfrak{s l}_{2}$-algebra. 


\subsection{Shifted symmetric functions and hook-length moments}

The shifted symmetric functions and hook-length moments are functions on partitions for which the $q$-bracket, defined by (1.2), is a quasimodular form; this is the content of the Bloch-Okounkov theorem (Theorem 1.1.2). Before defining these functions, note that it is not surprising at all that there are functions $f: \mathscr{P} \rightarrow \mathbb{Q}$ whose $q$-bracket $\langle f\rangle_{q}$ is a quasimodular form, since it is easily seen that the $q$-bracket from $\mathbb{Q}^{\mathscr{P}}$ to $\mathbb{Q}[[q]]$ is surjective. What is surprising is that one can find subalgebras $A$ of $\mathbb{Q}^{\mathscr{P}}$ which (i) are 'interesting' in the sense that they have an interpretation in combinatorics, enumerative geometry or others fields of mathematics, (ii) have the property that $\langle f\rangle_{q} \in \widetilde{M}$ for all $f \in A$ and (iii) are closed under multiplication. Observe that for such an algebra the $q$-bracket is not an algebra homomorphism, as the $q$-bracket is not multiplicative.

We think of a partition $\lambda$ in the following ways:

- A finite non-increasing sequence $\left(\lambda_{1}, \lambda_{2}, \ldots, \lambda_{\ell}\right)$ of positive integers, where $\ell=\ell(\lambda)$ is the length of $\lambda$;

- An infinite non-increasing sequence $\left(\lambda_{1}, \lambda_{2}, \ldots\right)$ of integers stabilising to 0 ;

- A Young diagram $Y_{\lambda}$;

- A multiset of integers $m$, where $r_{m}(\lambda)$ denotes the multiplicity of $m$ in the multiset;

- A finite set of modified Frobenius coordinates

$$
C_{\lambda}=\left\{-b_{1}, \ldots,-b_{r}, a_{r}, \ldots, a_{1}\right\},
$$

where $r$ is the number of boxes on the main diagonal in the Young diagram $Y_{\lambda}$, $a_{i}=\lambda_{i}-i+\frac{1}{2}$ is the number of boxes to the right of the diagonal in row $i$ (including $\frac{1}{2}$ for the box in row $i$ and column $i$, see Fig. 1.1 below) and $b_{i}=$ $\lambda_{i}^{\prime}-i+\frac{1}{2}$ is the number of boxes below the diagonal in column $i$. Here, $\lambda^{\prime}$ denotes the conjugate of $\lambda$ corresponding to transposing $Y_{\lambda}$.
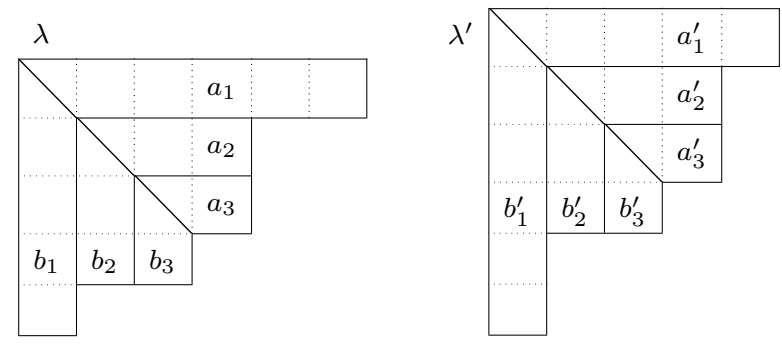

Figure 1.1: The modified Frobenius coordinates of $\lambda=(6,4,4,3,1)$ and $\lambda^{\prime}$. 
Definition 1.3.1. Let $\lambda \in \mathscr{P}$. Define the shifted symmetric polynomials $Q_{k}$ by $Q_{0}(\lambda)=1$ and for $k \geq 1$

$$
Q_{k}(\lambda)=\beta_{k}+\frac{1}{(k-1) !} \sum_{i=1}^{\infty}\left(\left(\lambda_{i}-i+\frac{1}{2}\right)^{k-1}-\left(-i+\frac{1}{2}\right)^{k-1}\right)
$$

with $\beta_{k}$ defined by

$$
\sum_{k=0}^{\infty} \beta_{k} z^{k-1}:=\frac{1}{2 \sinh (z / 2)} .
$$

The first three shifted symmetric polynomials $Q_{i}$ are given by

$$
Q_{0}(\lambda)=1, \quad Q_{1}(\lambda)=0, \quad Q_{2}(\lambda)=|\lambda|-\frac{1}{24} .
$$

In terms of the modified Frobenius coordinates of a partition, $Q_{k}$ turns out to be given by

$$
Q_{k}(\lambda)=\beta_{k}+\frac{1}{(k-1) !} \sum_{c \in C_{\lambda}} \operatorname{sgn}(c) c^{k-1} .
$$

Since $C_{\lambda}=-C_{\lambda^{\prime}}$, the shifted symmetric functions are invariant (up to a sign) under the conjugation $\lambda \mapsto \lambda^{\prime}$, that is,

$$
Q_{k}\left(\lambda^{\prime}\right)=(-1)^{k} Q_{k}(\lambda) .
$$

The algebra of shifted symmetric functions $\Lambda^{*}$, which is sometimes called the Bloch-Okounkov algebra, is the algebra generated by the $Q_{k}$ for $k \geq 2$, i.e.

$$
\Lambda^{*}=\mathbb{Q}\left[Q_{2}, Q_{3}, \ldots\right] .
$$

This algebra $\Lambda^{*}$ (i) admits a weight grading by assigning weight $k$ to $Q_{k}$ and (ii) is freely generated by the $Q_{k}$. Hence, $\Lambda^{*}$ is not finitely generated, although the subspace of a certain weight $k$ has a finite basis. In Chapter 2 we discuss certain differential operators on $\Lambda^{*}$ so that $\Lambda^{*}$ becomes a differential algebra, or rather an $\mathfrak{s l}_{2}$-algebra. We will see that these operators are more naturally defined on $\mathcal{R}=\mathbb{Q}\left[Q_{1}, Q_{2}, \ldots\right]$ (here we distinguish $Q_{k}$, which is now thought of as an independent abstract variable, from its image in $\mathbb{Q}^{\mathscr{P}}$ ). For example, the operator $\boldsymbol{\partial}$ can now be defined on $\mathcal{R}$ by $\boldsymbol{\partial}\left(Q_{k}\right)=Q_{k-1}$ for $k \geq 1$ and $\boldsymbol{\partial}\left(Q_{0}\right)=0$, which would not make sense on $\Lambda^{*}$.

Remark 1.3.2. Shifted symmetric polynomials were originally introduced by Okounkov and Olshanski as the following analogue of symmetric polynomials [OO97]. Given $m \geq 0$, write $\mathfrak{S}_{m}$ for the symmetric group on $m$ letters and let $\Lambda^{*}(m)$ be the space of rational polynomials in $m$ variables $x_{1}, \ldots, x_{m}$ which are shifted symmetric, i.e. invariant under the action of all $\sigma \in \mathfrak{S}_{m}$ given by 
$x_{i} \mapsto x_{\sigma(i)}+i-\sigma(i)$ (or more symmetrically $x_{i}-i \mapsto x_{\sigma(i)}-\sigma(i)$ ). Note that $\Lambda^{*}(m)$ is filtered by the total degree of the polynomials. We have forgetful maps $\Lambda^{*}(m) \rightarrow \Lambda^{*}(m-1)$ given by $x_{m} \mapsto 0$, so that we can define the space of shifted symmetric polynomials $\Lambda^{*}$ as the inverse limit

$$
\Lambda^{*}=\lim _{m} \Lambda^{*}(m)
$$

in the category of filtered algebras. Considering a partition $\lambda$ as a non-increasing sequence $\left(\lambda_{1}, \lambda_{2}, \ldots\right)$ of non-negative integers $\lambda_{i}$, we can interpret $\Lambda^{*}$ as being a subspace of all functions $\mathscr{P} \rightarrow \mathbb{Q}$.

A different, though (as we will see in a moment) closely related, family of functions on partitions is given by the hook-length moments. For a cell $\xi=(i, j)$ in the Young diagram $Y_{\lambda}$ (here $1 \leq i \leq \ell(\lambda)$ and $1 \leq j \leq \lambda_{i}$ ) we denote by

$$
h(\xi)=\left(\lambda_{i}-i\right)+\left(\lambda_{j}^{\prime}-j\right)+1
$$

the hook-length of $\xi$, i.e., the number of boxes to the right and below this box plus 1 for the box itself. Then, for $k \geq 2$ the hook-length moments are given by

$$
H_{k}(\lambda)=-\frac{B_{k}}{2 k}+\sum_{\xi \in Y_{\lambda}} h(\xi)^{k-2} .
$$

From [CMZ18] (Theorem 13.5, recalled below), it follows that the even hooklength moments are shifted symmetric functions. Write $e(x)=e^{2 \pi \mathrm{i} x}$. Then, this result can be expressed in terms of the generating series $V_{\lambda}(z)$ of hook-length moments and the generating series $W_{\lambda}(z)$ of shifted symmetric functions:

$$
\begin{aligned}
V_{\lambda}(z) & :=\frac{1}{(2 \pi \mathrm{i} z)^{2}}+2 \sum_{\substack{k \geq 2 \\
\text { even }}} H_{k}(\lambda) \frac{(2 \pi \mathrm{i} z)^{k-2}}{(k-2) !} \\
W_{\lambda}(z) & :=\sum_{k=0}^{\infty} Q_{k}(\lambda)(2 \pi \mathrm{i} z)^{k-1}=\sum_{i=1}^{\infty} e\left(\left(\lambda_{i}-i+\frac{1}{2}\right) z\right),
\end{aligned}
$$

where the first sum in (1.13) can be thought of as either a formal Laurent series in $z$ or a convergent power series for $z$ near 0 , but the second sum has to be interpreted as a convergent sum (and hence holomorphic function) in the lower half-plane.

Theorem 1.3.3. For all $\lambda \in \mathscr{P}$

$$
V_{\lambda}(z)=-W_{\lambda}(z) W_{\lambda}(-z),
$$


that is, for all positive even $k$ one has

$$
\frac{H_{k}(\lambda)}{(k-2) !}=\frac{1}{2} \sum_{i=0}^{k}(-1)^{i} Q_{i}(\lambda) Q_{k-i}(\lambda) .
$$

It follows that the algebra of even hook-length moments is a subalgebra of $\Lambda^{*}$ and hence that $\langle f\rangle_{q} \in \widetilde{M}$ for all $f$ in this algebra.

Definition 1.3.4. We say that an algebra $A$ of functions on partitions is maximal quasimodular if $\langle f\rangle_{q} \in \widetilde{M}$ for all $f \in A$ and $A$ is maximal with this property.

It is believed that $\Lambda^{*}$ is maximal quasimodular. In contrast, the algebra of even hook-length moments is not maximal quasimodular, as all shifted symmetric functions of odd weight are not contained in this algebra.

\subsection{The Jacobi theta series and the Bloch-Okounov theorem}

The Bloch-Okounkov theorem as stated by Theorem 1.1.2 does not determine which quasimodular form corresponds to some element of $\Lambda^{*}$. We recall a result by Zagier, as well as the original statement of Bloch and Okounkov, by which the $q$-bracket of $f \in \Lambda^{*}$ can be expressed as an explicit quasimodular form. This quasimodular form is given in terms of the Taylor coefficients of the Jacobi theta series $\theta$, given by

$$
\theta(\tau, z)=\sum_{\nu \in \mathbb{F}}(-1)^{\lfloor\nu\rfloor} e^{\nu z} q^{\nu^{2} / 2} \quad\left(\mathbb{F}=\mathbb{Z}+\frac{1}{2}, q=e^{2 \pi \mathrm{i} \tau}\right) .
$$

Often, we omit the dependence on $\tau$ and simply write $\theta(z)$ for $\theta(\tau, z)$.

By [Zag16, Theorem 1] one has the following identity, which determines the $q$-bracket recursively for all shifted symmetric functions.

Theorem 1.4.1. For $f \in \mathbb{Q}\left[Q_{2}, Q_{3}, \ldots\right]$

$$
\langle\theta(\boldsymbol{\partial}) f\rangle_{q}=0,
$$

where the $q$-bracket is extended to a map $\mathbb{C}[[q]]^{\mathscr{P}} \rightarrow \mathbb{C}[[q]]$ by linearity and $\boldsymbol{\partial}$ is defined on $p .8$.

Given integers $k_{1}, \ldots, k_{n}$, the shifted symmetric function $Q_{k_{1}} \cdots Q_{k_{n}}$ occurs (up to a constant) in $W\left(z_{1}\right) \cdots W\left(z_{n}\right)$ as the coefficient of $z_{1}^{k_{1}-1} \cdots z_{n}^{k_{n}-1}$ (recall $W_{\lambda}$ is the generating series of the shifted symmetric functions; see (1.13)). The 
second result expresses the $q$-bracket of this $n$-fold product in terms of the Jacobi theta function and its derivatives

$$
\theta^{(r)}(\tau, z):=\theta^{(r)}(z):=\frac{\partial^{r}}{\partial z^{r}} \theta(z) \quad(r \geq 0),
$$

in the following way ([BO00, p. 30], with a sign corrected as in [Zag16, Section 6]).

Definition 1.4.2. For all $n \geq 1$, let $\mathfrak{S}_{n}$ be the symmetric group on $n$ letters and define the Bloch-Okounkov n-point functions by

$$
F_{n}\left(\tau, z_{1}, \ldots, z_{n}\right)=\sum_{\sigma \in \mathfrak{S}_{n}} V_{n}\left(\tau, z_{\sigma(1)}, \ldots, z_{\sigma(n)}\right),
$$

where the functions $V_{n}$ are defined recursively by $V_{0}(\tau)=1$ and

$$
\sum_{m=0}^{n} \frac{(-1)^{n-m}}{(n-m) !} \theta^{(n-m)}\left(\tau, 2 \pi \mathrm{i}\left(z_{1}+\ldots+z_{m}\right)\right) V_{m}\left(\tau, z_{1}, \ldots, z_{m}\right)=0 .
$$

Theorem 1.4.3. With $W$ as in (1.13) and $F_{n}$ as in Definition 1.4.2, for all $n \geq 1$

$$
\left\langle W\left(z_{1}\right) \cdots W\left(z_{n}\right)\right\rangle_{q}=F_{n}\left(\tau, z_{1}, \ldots, z_{n}\right) .
$$

\subsection{Motivation from enumerative geometry}

Elliptic curves and (quasi)modular forms are connected in various ways. A first connection is made by the Weierstrass $\wp$-function, given by

$$
\wp(\tau, z):=\frac{1}{z^{2}}+\sum_{\substack{\omega \in \mathbb{Z} \tau+\mathbb{Z} \\ \omega \neq 0}}\left(\frac{1}{(z+\omega)^{2}}-\frac{1}{\omega^{2}}\right) \quad(\tau \in \mathfrak{h}, z \in \mathbb{C}) .
$$

On the one hand, the Weierstrass $\wp$-function parametrises elliptic curves over $\mathbb{C}$; on the other hand, the coefficients in its Laurent series are modular forms:

$$
\wp(\tau, z)=\frac{1}{z^{2}}+\sum_{k \geq 3}(k-1) e_{k} z^{k-2} .
$$

A much deeper connection between elliptic curves and modular forms is made by the modularity theorem, by which every elliptic curve over $\mathbb{Q}$ can be parametrised by modular functions.

In this section we motivate the results in this thesis by a third connection: various counting problems on elliptic curves give rise to shifted symmetric functions and 
quasimodular forms. This is in fact the beginning of a whole story: Dijkgraaf had given a physical argument, which was proven by Kaneko and Zagier, that the problem of counting certain coverings of a torus led to quasimodular forms, and the BlochOkounkov theorem was found as a generalisation of this [Dij95, KZ95, BO00]. Some results in this direction will be described in the rest of this section; more recent results can, e.g., be found in [BKY18, CMSZ20, GM20].

\section{Hurwitz theory}

Let $E$ be an elliptic curve over $\mathbb{C}$ (i.e., $E$ is a complex torus). The Hurwitz theory of $E$ is concerned with the enumeration of covering maps

$$
\pi: X \rightarrow E
$$

of a fixed degree $d$ and fixed ramification profile (a tuple of partitions determining the ramification at the ramification points). Here, $X$ is a Riemann surface whose genus is uniquely determined by this degree and profile. Hurwitz numbers are defined as a certain weighted count of the number of such mappings up to isomorphism, defined by Definition 3.2.1. For example, the aformentioned result of [Dij95, KZ95] are on generating series of simple Hurwitz numbers over an elliptic curve, i.e., corresponding to mappings $\pi$ for which the preimage of a point in $E$ consists of at least $d-1$ points (see the example below).

This result was generalized in the following way. For any ramification profile the Hurwitz numbers can naturally be expressed in terms of shifted symmetric functions. From this it follows that the generating series of all ramified coverings of $E$ by a Riemann surface of genus $g>1$ and a fixed ramification profile is a quasimodular form of mixed weight $\leq 6 g-6$ for $\mathrm{SL}_{2}(\mathbb{Z})$ [EO01, Och01] (see Chapter 3 for a proof and a generalisation of this result).

Example 1.5.1. Suppose all ramification is simple, i.e., the preimage of a point in $E$ consists of at least $d-1$ points. Then, the Hurwitz number corresponding to degree $d$ coverings of $E$ by a Riemann surface $X$ of genus $g$ is given by

$$
\sum_{|\lambda|=d} Q_{3}(\lambda)^{2 g-2}
$$

For example, for $g=2$ one finds

$$
\left\langle Q_{3}^{2}\right\rangle_{q}=\frac{1}{1440}\left(D Q+10 D^{2} P\right),
$$

from which it follows that there are $\frac{1}{6} d\left(\sigma_{3}(d)-d \sigma(d)\right)$ simple degree $d$ coverings of a Riemann surface of genus 2 to $E$. 
There is a second enumerative theory of mappings from curves to curves, namely Gromov-Witten theory. In fact, the stationary sector of Gromov-Witten theory and Hurwitz theory are equivalent [OP06]. Moreover, the corresponding generating series of Gromov-Witten invariants are actual quasimodular forms of weight $6 g-6$ (and not merely quasimodular forms of mixed weights).

\section{Flat surfaces}

A flat surface $X$ is a Riemann surface with a flat metric, where we allow a finite number of singular points. For example, given a ramified covering $\pi: X \rightarrow E$ as in the previous section, pulling back the (flat) metric on the torus yields a flat metric on $X$, with singularities at the ramification points. The number of closed geodesics of bounded length on such a surface is determined by a weighted version of the Hurwitz numbers. The generating series of $p$ th weighted Hurwitz number can be expressed by inserting a hook-length moment $H_{p+1}$ in the formula for ordinary Hurwitz numbers. In particular, these generating series are quasimodular forms of mixed weight [CMZ18, Theorem 6.4].

Example 1.5.2. Consider flat surfaces with only simple singularities. The corresponding generating series of $p$-weighted Hurwitz numbers is given by

$$
\left\langle Q_{3}^{2 g-2} H_{p+1}\right\rangle_{q},
$$

which is a quasimodular form of weight $6 g-6+p+1$. The key fact for the application to flat surfaces is that this conclusion even holds for $p=-1$, whereas $H_{0} \notin \Lambda^{*}$; hence, note that this does not follow from a direct application of the Bloch-Okounkov theorem. 


\subsection{Main questions and results}

In this thesis, our aim is to study graded algebras of functions on partitions such that all homogeneous elements of the algebra have quasimodular forms as $q$-brackets. That is, we now refine Question 1.1.1 to the following three main questions discussed in this thesis:

(1) Are there graded algebras $A \nsubseteq \Lambda^{*}$ such that for all homogeneous $f \in A$ the $q$-bracket $\langle f\rangle_{q}$ is quasimodular for $\mathrm{SL}_{2}(\mathbb{Z})$ ?

(2) Given a congruence subgroup $\Gamma \leq \mathrm{SL}_{2}(\mathbb{Z})$, is there an (even larger) algebra of functions for which the $q$-bracket is a quasimodular form for $\Gamma$ ?

(3) What is the class of functions for which the $q$-bracket is not only a quasimodular form, but even a modular form?

These questions are discussed and answered in the three parts of this thesis. In Part I we motivate these three questions by answering Question (3) for the BlochOkounkov algebra and expanding on the motivation from enumerative geometry. Question (1) is answered affirmatively in Part II. Finally, in Part III we explain how to answer Questions (2) and (3) by studying a different question of independent interest (motivated by the results in Section 1.4):

(4) What is the modular or quasimodular behaviour of the Taylor coefficients of a meromorphic quasi-Jacobi form?

We now state our main results (Theorem A-H below), without presenting the most technical version of these results.

\section{Results on the Bloch-Okounkov theorem}

In Chapter 2 we answer Question (3) for the Bloch-Okounkov algebra. By [Zag16] there is a second order differential operator $\Delta$ such that the diagram

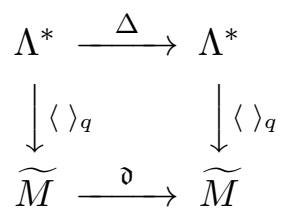

commutes. From this diagram, we see that the $q$-brackets of elements in the space $\mathcal{H}:=\operatorname{ker} \Delta$ are in the kernel of $\mathfrak{d}$, i.e., they are modular forms. The following theorem gives the converse statement. This result motivates the study of the $\mathfrak{s l}_{2}$ action on quasimodular forms, as well as on functions of partitions. 
Theorem A (Theorem 2.1.3, Theorem 2.1.4 and Theorem 2.1.6). The space $\Lambda^{*}$ has a direct sum decomposition as

$$
\Lambda^{*}=\mathcal{H} \oplus Q_{2} \Lambda^{*}
$$

given by an explicitly computable projection $\pi: \Lambda^{*} \rightarrow \mathcal{H}$ with kernel $Q_{2} \Lambda^{*}$, and

$$
M \cap\left\langle Q_{2} \Lambda^{*}\right\rangle_{q}=\{0\} .
$$

The projection $\pi$ is computable by formulas for the canonical basis $\left\{h_{\lambda}\right\}$ of $\mathcal{H}$ via $h_{\lambda}=\pi\left(Q_{\lambda_{1}} \cdots Q_{\lambda_{n}}\right)$, where $\lambda$ runs over all partitions with all parts at least 3 .

In particular, by using (1.16) inductively, each $f \in \Lambda^{*}$ can uniquely be written as a polynomial in $Q_{2}$ with coefficients $h_{i} \in \mathcal{H}$, i.e.,

$$
f=\sum_{i \geq 0} h_{i} Q_{2}^{i} .
$$

As $\langle g\rangle_{q}=0$ if and only if $\left\langle Q_{2} g\right\rangle_{q}=0$ for $g \in \Lambda^{*}$, the following are equivalent:

(i) $\langle f\rangle_{q}$ is modular;

(ii) $\langle f\rangle_{q}=\left\langle h_{0}\right\rangle_{q}$;

(iii) $\left\langle h_{i}\right\rangle_{q}=0$ for all $i>0$.

We will prove this theorem in Chapter 2 by an explicit construction of the projection map inspired by the well-known projection map from polynomials to their harmonic parts; a second proof will also follow from the results of Chapter 5 on quasi-Jacobi forms, described below.

Next, we motivative Question (1) by applying the Bloch-Okounkov theorem to a counting problem originating from tropical geometry. More precisely, we study the generating series of so-called triply mixed Hurwitz numbers, which can be expressed in terms of the representation theory of the symmetric group. In this result, the (multiplicative) algebra structure of the Bloch-Okounkov algebra is essential.

Theorem B ( = Theorem 3.4.4). For every type of triply mixed Hurwitz numbers $h_{d}$ of degree d over an elliptic curve, the corresponding generating series $\sum_{d=1}^{\infty} h_{d} q^{d}$ is a quasimodular form of mixed weight. 


\section{A symmetric Bloch-Okounkov theorem}

In Chapter 4 we give an affirmative answer to Question (1). For positive $k$ define the (part) moment functions $S_{k}$ by

$$
S_{k}(\lambda)=-\frac{B_{k}}{2 k}+\sum_{i=1} \lambda_{i}^{k-1}=-\frac{B_{k}}{2 k}+\sum_{m=1}^{\infty} m^{k-1} r_{m}(\lambda),
$$

where $r_{m}(\lambda)$ denotes the number of parts of size $m$ in $\lambda$. Let $\mathcal{S}$ be the graded algebra generated by the moment functions $S_{k}$ for even $k$. We will show that $\langle f\rangle_{q} \in \widetilde{M}$ for all $f \in \mathcal{S}$, even though $\mathcal{S}$ is not contained in $\Lambda^{*}$. However, $\mathcal{S}$ is not maximal quasimodular in the sense of Definition 1.3.4, since we will construct a larger graded algebra $\mathcal{T}$ generated by certain functions $T_{k, l}$. These functions $T_{k, l}$ are defined as a sum over $m$ of a polynomial in $m$ and $r_{m}(\lambda)$ (see (4.2)). In particular, $T_{k, 1}=S_{k+1}$.

Theorem $\mathbf{C}$ ( $=$ Theorem 4.1.2). For all homogeneous $f \in \mathcal{T}$ the $q$-bracket $\langle f\rangle_{q}$ is a quasimodular form of the same weight as $f$.

The key idea in this construction, and in most of the proofs in Chapter 4, is the principle to establish all identities in $\mathbb{Q}^{\mathscr{P}}$ or $\mathcal{T}$ before taking the $q$-bracket, instead of doing these computations in $\mathbb{Q}[[q]]$ or the space of quasimodular forms $\widetilde{M}$. By doing so, we discover the algebraic structure of $\mathcal{T}$. For example, this principle is applied in the following situations:

\begin{tabular}{ll} 
previous definitions and results & new definitions and results \\
\hline multiplication in $\mathbb{Q}[[q]]$ & induced product $\odot$ on $\mathbb{Q}^{\mathscr{P}}$ \\
\hline -bracket: $\mathbb{Q}^{\mathscr{P}} \rightarrow \mathbb{Q}[[q]]$ & u-bracket: $\mathbb{Q}^{\mathscr{P}} \rightarrow \mathbb{Q}^{\mathscr{}}\left[\left[u_{1}, u_{2}, \ldots\right]\right]$ \\
\hline derivative $q \mathrm{~d} \mathrm{~d} q$ on $\mathbb{Q}[[q]]$ & derivative on $\mathbb{Q}^{\mathscr{P}}$ \\
\hline $\mathfrak{s l}_{2}$-action on $\widetilde{M}$ & $\mathfrak{s l}_{2}$-action on $\mathcal{T}$ \\
\hline Rankin-Cohen brackets on $\widetilde{M}$ & Rankin-Cohen brackets on $\mathcal{T}$. \\
\hline
\end{tabular}




\section{Quasi-Jacobi forms and applications}

Recall that the $q$-bracket of $W\left(z_{1}\right) \cdots W\left(z_{n}\right)$ equals $F_{n}\left(z_{1}, \ldots, z_{n}\right)$, which can be expressed in terms of the Jacobi theta function. (See Theorem 1.4.3.) In fact, $F_{n}$ is an example of a quasi-Jacobi form, a concept we introduce in Chapter 5. Quasi-Jacobi forms transform comparable to quasimodular forms (see Equation 1.6), as we explain now. There is a slash action on all functions $\varphi: \mathfrak{h} \times \mathbb{C}^{n} \rightarrow \mathbb{C}$ for all $\gamma \in \mathrm{SL}_{2}(\mathbb{Z})$ and for all $X \in M_{2, n}(\mathbb{Q})$ (so actually for the action of their semidirect product $\mathrm{SL}_{2}(\mathbb{Z}) \ltimes M_{2, n}(\mathbb{Q})$ ). In case $\varphi$ is a quasi-Jacobi form of weight $k$ and index $M$, there exist quasi-Jacobi forms $\varphi_{i, j}$, indexed by a finite subset of $\mathbb{Z}_{\geq 0} \times \mathbb{Z}_{\geq 0}^{n}$, such that for all $\gamma=\left(\begin{array}{ll}a & b \\ c & d\end{array}\right)$ and $X=\left(\begin{array}{l}\boldsymbol{\lambda} \\ \boldsymbol{\mu}\end{array}\right) \in M_{2, n}(\mathbb{Z})$ one has

$$
\begin{aligned}
\left(\left.\varphi\right|_{k, M} \gamma\right)\left(\tau, z_{1}, \ldots, z_{n}\right) & =\sum_{i, \boldsymbol{j}} \varphi_{i, j}\left(\tau, z_{1}, \ldots, z_{n}\right)\left(\frac{c}{c \tau+d}\right)^{i+|\boldsymbol{j}|} z_{1}^{j_{1}} \cdots z_{n}^{j_{n}}, \\
\left(\left.\varphi\right|_{M} X\right)\left(\tau, z_{1}, \ldots, z_{n}\right) & =\sum_{\boldsymbol{j}} \varphi_{0, \boldsymbol{j}}\left(\tau, z_{1}, \ldots, z_{n}\right)\left(-\lambda_{1}\right)^{j_{1}} \cdots\left(-\lambda_{n}\right)^{j_{n}}
\end{aligned}
$$

together with similar formulas for each $\left.\varphi_{i, \boldsymbol{j}}\right|_{k, M} \gamma$ and $\left.\varphi_{i, \boldsymbol{j}}\right|_{M} X$. Jacobi forms are quasi-Jacobi forms for which the right-hand side in both equations above equals $\varphi$.

The quasi-Jacobi forms $\varphi$ we study are strictly meromorphic, i.e., meromorphic such that if $\boldsymbol{z} \in \mathbb{R}^{n} \tau+\mathbb{R}^{n}$ is a pole of $\varphi(\tau, \cdot)$ for some $\tau \in \mathfrak{h}$, it is a pole for almost all $\tau \in \mathfrak{h}$ (see Section 5.6). The Weierstrass $\wp$-function is an example of a strictly meromorphic Jacobi form, but its multiplicative inverse is not. In case $n=1$, this condition is equivalent to the statement that all poles of $\varphi$ are torsion points $z \in \mathbb{Q} \tau+\mathbb{Q}$. This is crucial in order to obtain mock modular forms as Fourier coefficients of meromorphic Jacobi forms (see [Zwe02, DMZ14]). For $n>1$ the Jacobi transformation properties of $\varphi$ imply a more complicated restriction on the positions of the poles:

Theorem D (= Theorem 5.4.2). Let $\varphi$ be a strictly meromorphic quasi-Jacobi form and $\tau \in \mathfrak{h}$. Then, all poles $\boldsymbol{z}$ of $\varphi(\tau, \cdot)$ lie in finite union of rational hyperplanes

$$
s_{1} z_{1}+\ldots+s_{n} z_{n} \in p \tau+q
$$

with $s_{1}, \ldots, s_{n} \in \mathbb{Z}$ and $p, q \in \mathbb{Q} / \mathbb{Z}$.

We give a detailed description of the Taylor coefficients (or rather Laurent coefficients in case we are expanding around a pole) of a strictly meromorphic quasi-Jacobi form. This is the technical result we need in order to answer Question (2) and (3). For simplicity, we assume that $s$ in the result above is always a standard basis vector, e.g., we allow $\wp\left(z_{1}\right) \wp\left(z_{2}+\frac{1}{2}\right)$, but we do not allow $\wp\left(z_{1}-z_{2}\right)$. 
Given $X \in M_{2, n}(\mathbb{Q})$, the congruence subgroup $\Gamma_{X} \leq \mathrm{SL}_{2}(\mathbb{Z})$ is defined by Equation (5.17) in such a way that

$$
\left.\left.\varphi\right|_{M} X\right|_{k, M} \gamma=\left.\varphi\right|_{M} X \quad\left(\gamma \in \Gamma_{X}\right)
$$

for Jacobi forms $\varphi$ of weight $k$ and index $M$.

Theorem E ( = Theorem 5.9.10). Let $\varphi$ be a strictly meromorphic quasi-Jacobi form of weight $k$ and index $Q$ whose poles $\boldsymbol{z}$ lie on a finite collection of hyperplanes of the form $z_{s} \in p \tau+q$ with $s \in\{1, \ldots, n\}$ and $p, q \in \mathbb{Q} / \mathbb{Z}$. Then

(i) for all $X \in M_{2, n}(\mathbb{Q})$ and $\ell \in \mathbb{Z}^{n}$ the 'Taylor coefficients' $g_{\ell}^{X}(\varphi)$, defined by Definition 5.9.2, are quasimodular forms of weight $k+|\ell|$ for the group $\Gamma_{X}$ and satisfy the functional equations (5.20) and (5.21).

(ii) for all $X \in M_{2, n}(\mathbb{Q})$ and $\boldsymbol{m} \in \mathbb{Z}^{n}$ the combinations $\xi_{m}^{X}(\varphi)$ of the derivatives of $g_{\ell}^{X}(\varphi)$, defined by (5.19), are modular forms of weight $k+|\boldsymbol{m}|$ for $\Gamma_{X}$ and satisfy the functional equation (5.20).

Definition 5.9.2 and Equation (5.19) use the functions $\varphi_{i, j}$ in (1.17) and (1.18), which are uniquely determined by the quasi-Jacobi form $\varphi$. The results of Chapter 5 also show that the four collections of functions $\{\varphi\},\left\{\varphi_{i, j}\right\},\left\{g_{\ell}^{X}\right\}$ and $\left\{\xi_{m}^{X}\right\}$ determine each other in a computable way, and give explicit conditions on the collections $\left\{\varphi_{i, j}\right\},\left\{g_{\ell}^{X}\right\}$ and $\left\{\xi_{m}^{X}\right\}$ that imply that they arise from a quasi-Jacobi form.

Applying property (i) in Theorem E to suitable strictly meromorphic quasi-Jacobi forms yields an answer to Question (2). For example, given $a \in \mathbb{Q}, k \geq 1$ and $\lambda \in \mathscr{P}$, let $Q_{k}(\lambda, a)$ be given by

$$
\beta_{k}(a)+\frac{1}{(k-1) !} \sum_{i=1}^{\infty}\left(\boldsymbol{e}(a)^{\lambda_{i}-i}\left(\lambda_{i}-i+\frac{1}{2}\right)^{k-1}-\boldsymbol{e}(a)^{-i}\left(-i+\frac{1}{2}\right)^{k-1}\right),
$$

where $\sum_{k \in \mathbb{Z}} \beta_{k}(a)(2 \pi \mathrm{i} z)^{k-1}=\frac{\boldsymbol{e}(z / 2)}{\boldsymbol{e}(z+a)-1}$ and $\boldsymbol{e}(x)=e^{2 \pi \mathrm{i} x}$. The main properties satisfied by these functions are a consequence of the fact that

$$
\sum_{k \geq 0} Q_{k}(\lambda, a)(2 \pi \mathrm{i} z)^{k-1}=\boldsymbol{e}\left(-\frac{1}{2} a\right) W_{\lambda}(z+a)
$$

and that $F_{n}\left(z_{1}, \ldots, z_{n}\right)=\left\langle W\left(z_{1}\right) \cdots W\left(z_{n}\right)\right\rangle_{q}$ is a quasi-Jacobi form. It is by applying Theorem E (i) to this function that we deduce the following result on the quasimodularity of the $q$-bracket of polynomials in the functions $Q_{k}(\cdot, a)$.

Let the algebra $\Lambda^{*}(N) \subseteq \overline{\mathbb{Q}}^{\mathscr{P}}$ be given by

$$
\Lambda^{*}(N):=\mathbb{Q}\left[Q_{k}(\cdot, a) \mid k \geq 1, a \in\left\{0, \frac{1}{N}, \ldots, \frac{N-1}{N}\right\}\right]
$$

and graded by assigning weight $k$ to $Q_{k}(\cdot, a)$. 
Theorem $\mathbf{F}$ ( $=$ Theorem 6.1.1). Let $k \in \mathbb{Z}, N \geq 1$ odd. Then, for $f \in \Lambda^{*}(N)$ of weight $k$ the function $\langle f\rangle_{q}$, after rescaling $\tau$ by a factor of $N$, is a quasimodular form of weight $k$ and level $N$.

In Chapter 6 we state and prove the above result in a more general setting, which has applications to the hook-length moments and moment functions.

As the $n$-point functions $F_{n}$ corresponding to the Bloch-Okounkov algebra $\Lambda^{*}$ are quasi-Jacobi forms, by Theorem E(ii) we find an infinite family of modular forms. Finding a suitable inverse under the $q$-bracket for these functions recovers the answer to Question (3) given in Theorem A. We generalise this statement to other algebras for which the corresponding $n$-point functions are quasi-Jacobi forms:

Theorem $\mathbf{G}$ ( $=$ Theorem 7.2.3). For any algebra $\mathcal{F}$ of functions on partitions satisfying the conditions in Section 7.2, there exists a subspace $\mathcal{M}$ of $\mathcal{F}$ such that Theorem $A$ holds after replacing $\Lambda^{*}$ by $\mathcal{F}$ and $\mathcal{H}$ by $\mathcal{M}$.

In particular, since $\mathcal{F}=\Lambda^{*}$ satisfies the conditions in question, by proving Theorem $\mathrm{G}$ we obtain a second proof of Theorem A. In Chapter 7 we explain how this result answers the question "when is the $q$-bracket modular?", not only for the shifted symmetric functions, but also for their generalisation to congruence subgroups and for the moment functions.

As a final application of the study of quasi-Jacobi forms, motivated by the study of the Gromov-Witten theory of $\mathrm{K} 3$ surfaces, for $m \in \mathbb{Z}$ we study the following differential equation:

$$
D_{\tau}^{2}\left(\varphi_{m}\right)=m^{2} F \varphi_{m},
$$

where $F(\tau, z):=\Theta(\tau, z)^{-1} D_{\tau}^{2} \Theta(\tau, z)$. This is a generalisation of the KanekoZagier equation (see (8.1)) that they found in connection with the study of supersingular $j$-invariants in finite characteristic, as recalled in Section 8.1. We also study the second order differential equation

$$
D_{\tau} \varphi_{m, n}=m n \varphi_{m} \varphi_{n} F+\left(D_{\tau} \varphi_{m}\right)\left(D_{\tau} \varphi_{n}\right)
$$

for $m, n \in \mathbb{Z}$. If the constant term in the Fourier expansion is fixed appropriately, the unique solutions are quasi-Jacobi forms.

Theorem $\mathbf{H}$ (Theorem 8.1.2 and Theorem 8.1.5). The solutions $\varphi_{m}$ and $\varphi_{m, n}$ of the two differential equations are quasi-Jacobi forms. In fact,

$$
\varphi_{m}=\operatorname{Res}_{x=0}\left(\frac{\Theta(x+z)}{\Theta(x)}\right)^{m} .
$$

Besides proving this result, in Chapter 8 we describe the transformation behaviour of the functions $\varphi_{m}$ and $\varphi_{m, n}$ under the Jacobi group. 



\section{Part I}

\section{Results on the \\ Bloch-Okounkov algebra}



CHAPTER 2

\section{When is the Bloch-Okounkov q-bracket modular?}

\subsection{Introduction}

Given a family of quasimodular forms, the question which of its members are modular often has an interesting answer. For example, consider the family of theta series

$$
\theta_{P}(\tau)=\sum_{\boldsymbol{x} \in \mathbb{Z}^{r}} P(\boldsymbol{x}) q^{x_{1}^{2}+\ldots+x_{r}^{2}} \quad\left(q=e^{2 \pi i \tau}\right)
$$

given by all homogeneous polynomials $P \in \mathbb{Z}\left[x_{1}, \ldots, x_{r}\right]$. Then, $\theta_{P}$ is a quasimodular form which is modular if and only if $P$ is harmonic (i.e., $P \in \operatorname{ker} \sum_{i=1}^{r} \partial^{2} / \partial x_{i}^{2}$ ) $[\mathrm{Sch} 39]^{1}$. Also, for every two modular forms $f, g$ one can consider the linear combination of products of derivatives of $f$ and $g$ given by

$$
\sum_{r=0}^{n} a_{r} D_{\tau}^{r} f D_{\tau}^{n-r} g \quad\left(a_{r} \in \mathbb{C}\right) .
$$

This linear combination is a quasimodular form which is modular precisely if it is a multiple of the Rankin-Cohen bracket $[f, g]_{n}$, given by (1.9). In this chapter, we provide a condition to decide which member of the family of quasimodular forms provided by the Bloch-Okounkov theorem is modular.

Recall that a quasimodular form $f$ is modular precisely if $\mathfrak{d} f=0$, where the operator $\mathfrak{d}$ is part of the $\mathfrak{s l}_{2}$-triple by which $\widetilde{M}$ becomes an $\mathfrak{s l}_{2}$-algebra. The results in [Zag16] allow us to extend this operator $\mathfrak{d}$ to an operator $\Delta^{*}$ acting on the shifted symmetric algebra $\Lambda^{*}$, and more generally to extend the $\mathfrak{s l}_{2}$-triple acting on quasimodular forms to an $\mathfrak{s l}_{2}$-triple acting on $\Lambda^{*}$. This operator $\Delta^{*}$ is not a derivation, hence this triple does not make $\Lambda^{*}$ into an $\mathfrak{s l}_{2}$-algebra (in the sense of Definition 1.2.4).

This chapter is based on [I20].

${ }^{1}$ As quasimodular forms were not yet defined in 1939 , Schoeneberg only showed that $\theta_{P}$ is modular if $P$ is harmonic. However, for every polynomial $P$ it follows that $\theta_{P}$ is quasimodular by decomposing $P$ as in Formula (2.1) below. 
Proposition 2.1.1. There exists actions of the Lie algebra $\mathfrak{s l}_{2}$ on both the algebra of shifted symmetric polynomials $\Lambda^{*}$ and the algebra of quasimodular forms $\widetilde{M}$ such that the $q$-bracket $\langle\cdot\rangle_{q}: \Lambda^{*} \rightarrow \widetilde{M}$ is $\mathfrak{s l}_{2}$-equivariant.

The answer to the question in the title of this chapter is provided by the operator $\Delta^{*}$. Namely, letting $\mathcal{H}=\operatorname{ker} \Delta^{*}$ and $I=\operatorname{ker}\langle\rangle_{q} \cap \Lambda^{*}$, we prove the following theorem (it is believed that the right-hand side $M \cap\left\langle\Lambda^{*}\right\rangle_{q}$ below simply equals $M$ ).

Theorem 2.1.2. The q-bracket yields an isomorphism

$$
(\mathcal{H}+I) / I \simeq M \cap\left\langle\Lambda^{*}\right\rangle_{q},
$$

i.e., for $f \in \Lambda^{*}$ the $q$-bracket $\langle f\rangle_{q}$ is modular if and only if $f=h+i$ with $h \in \mathcal{H}$ and $i \in I$.

The last section of this chapter is devoted to describing the graded algebra $\mathcal{H}$. We call $\mathcal{H}$ the space of shifted symmetric harmonic polynomials, as the description of this space turns out to be very similar to the space of classical harmonic polynomials. Let $\mathcal{P}_{d}^{(m)}$ be the space of homogeneous polynomials of degree $d$ in $m$ variables $x_{1}, \ldots, x_{m}$, let $\|x\|^{2}=\sum_{i} x_{i}^{2}$ and recall that the space $\mathscr{H}_{d}^{(m)}$ of degree $d$ homogeneous harmonic polynomials is given by $\operatorname{ker} \sum_{i=1}^{m} \partial^{2} / \partial x_{i}^{2}$. The main theorem about harmonic polynomials states that every polynomial $P \in \mathcal{P}_{d}^{(m)}$ can uniquely be written in the form

$$
P(\boldsymbol{x})=\sum_{0 \leq i \leq d / 2} h_{i}\|x\|^{2 i}
$$

with $h_{i} \in \mathscr{H}_{d-2 i}$. Here, $h_{0}$ is the canoncial projection of $P$ onto the space of harmonic polynomials. Define $K^{(m)}$, the Kelvin transform, and $D^{\alpha}$ for $\alpha$ an $m$-tuple of non-negative integers by

$$
f(x) \mapsto\|x\|^{2-m} f\left(\frac{x}{\|x\|^{2}}\right) \quad \text { and } \quad D^{\alpha}=\prod_{i=1}^{m} \frac{\partial_{i}^{\alpha}}{\partial x_{i}^{\alpha_{i}}} .
$$

An explicit basis for $\mathscr{H}_{d}^{(m)}$ if $m \geq 3$ is given by

$$
\left\{K^{(m)} D^{\alpha} K^{(m)}(1) \mid \alpha \in\{0,1\} \times \mathbb{Z}_{\geq 0}^{m-1}, \sum_{i} \alpha_{i}=d\right\} ;
$$

see, for example, [ABR01]. We prove the following analogous results for the space of shifted symmetric polynomials.

Theorem 2.1.3. Every $f \in \Lambda_{n}^{*}$ has a unique expansion

$$
f=\sum_{0 \leq i \leq n / 2} h_{i} Q_{2}^{i},
$$

where $h_{i} \in \mathcal{H}_{n-2 i}$. 
Theorem 2.1.4. The set

$$
\left\{\operatorname{pr} K \Delta_{\lambda} K(1) \mid \lambda \in \mathscr{P}(n), \text { all parts are at least } 3\right\}
$$

is a vector space basis of $\mathcal{H}_{n}$, where $\operatorname{pr}, K$ and $\Delta_{\lambda}$ are defined by Equation (2.4), Definition 2.3.6 and Definition 2.3.10, respectively.

Remark 2.1.5. If $\lambda$ is a partition with some part equal to 2 , the element $\Delta_{\lambda} K(1)$ vanishes as $\Delta_{2}=\Delta$ vanishes on harmonic functions. The reason we excluded $m=2$ in the basis (2.2) is different: $K^{(2)}$ is trivial.

Now, note that the first part of Theorem A follows from the above two results, as follows: the decomposition is given by Theorem 2.1.3, the projection map $\pi$ : $\Lambda^{*} \rightarrow \mathcal{H}$ is given by $f \mapsto h_{0}$ and is computable using Theorem 2.1.4 (a table of all basis elements of weight at most 10 can be found in Appendix A.1). The last part of Theorem A is implied by the following result.

Theorem 2.1.6. Write $f \in \Lambda^{*}$ as in (2.3). Then, the following are equivalent:

(i) $\langle f\rangle_{q}$ is modular;

(ii) $\langle f\rangle_{q}=\left\langle h_{0}\right\rangle_{q}$;

(iii) $\left\langle h_{i}\right\rangle_{q}=0$ for all $i>0$.

In particular, it follows that $f \in I$ if and only if $\left\langle h_{i}\right\rangle_{q}=0$ for all $i$.

Remark 2.1.7. The action of $\mathfrak{s l}_{2}$ given by Proposition 2.1.1 makes $\Lambda^{*}$ into an infinitedimensional $\mathfrak{s l}_{2}$-representation for which the elements of $\mathcal{H}$ are the lowest weight vectors. Theorem 2.1.3 is equivalent to the statement that $\Lambda^{*}$ is a direct sum of the (not necessarily irreducible) lowest weight modules

$$
V_{n}=\bigoplus_{m=0}^{\infty} Q_{2}^{m} \mathcal{H}_{n} \quad(n \in \mathbb{Z}) .
$$

\subsection{An $\mathfrak{s l}_{2}$-equivariant Bloch-Okounkov theorem}

We study differential operators on the algebra of shifted symmetric functions $\Lambda^{*}$. By doing so, at the end of this section we prove the $\mathfrak{s l}_{2}$-equivariant Bloch-Okounkov theorem (Proposition 2.1.1).

In order to define these differential operators, we let $\mathcal{R}=\mathbb{Q}\left[Q_{1}, Q_{2}, \ldots\right]$ with the $Q_{k}$ formal variables. This algebra has a representation on $\Lambda^{*}$ by sending the 
independent variable $Q_{k}$ to the function $Q_{k}$, where we hope that the use of the same letter will not create confusion. The space $\mathcal{R}$ has the following interpretation.

Observe that a non-increasing sequence $\left(\lambda_{1}, \lambda_{2}, \ldots\right)$ of integers corresponds to a partition precisely if it converges to 0 . If, however, it converges to an integer $n$, the equation

$$
\sum_{k=0}^{\infty} Q_{k}(\lambda) z^{k-1}:=\sum_{i=1}^{\infty} e^{\left(\lambda_{i}-i+\frac{1}{2}\right) z}
$$

still defines $Q_{k}(\lambda)$. In fact, in this case

$$
Q_{k}(\lambda)=\left(e^{n \boldsymbol{\partial}}\right) Q_{k}(\lambda-n)
$$

by [Zag16, Proposition 1] where $\partial Q_{0}=0, \partial Q_{k}=Q_{k-1}$ for $k \geq 1$ and $\lambda-n=$ $\left(\lambda_{1}-n, \lambda_{2}-n, \ldots\right)$ corresponds to a partition (i.e. converges to 0$)$. In particular $Q_{1}(\lambda)=n$ equals the number the sequence $\lambda$ converges to. The space $\mathcal{R}$ now has a faithful representation on the space of functions from non-increasing eventually constant sequences of integers to $\mathbb{Q}$, and by abuse of notations we will often identify $\mathcal{R}$ with its image under this latter map.

It is convenient to work with $\mathcal{R}$ instead of $\Lambda^{*}$ to define the differential operators $\Delta$ and more generally $\Delta_{\lambda}$ later. Both on $\Lambda^{*}$ and $\mathcal{R}$ we define a weight grading by assigning to $Q_{i}$ weight $i$. Denote the inclusion and projection maps by

$$
\iota: \Lambda^{*} \rightarrow \mathcal{R}, \quad \text { pr }: \mathcal{R} \rightarrow \Lambda^{*} .
$$

We extend $\langle\cdot\rangle_{q}$ to $\mathcal{R}$ via this projection map.

The operator $E=\sum_{m=0}^{\infty} Q_{m} \frac{\partial}{\partial Q_{m}}$ on $\mathcal{R}$ multiplies an element of $\mathcal{R}$ by its weight. Moreover, we consider the differential operators $\partial$ and $\mathscr{D}$ on $\mathcal{R}$ given by

$$
\boldsymbol{\partial}=\sum_{m=0}^{\infty} Q_{m} \frac{\partial}{\partial Q_{m+1}} \quad \text { and } \mathscr{D}=\sum_{k, \ell \geq 0}\left(\begin{array}{c}
k+\ell \\
k
\end{array}\right) Q_{k+\ell} \frac{\partial^{2}}{\partial Q_{k+1} \partial Q_{\ell+1}}
$$

Let $\Delta=\frac{1}{2}\left(\mathscr{D}-\partial^{2}\right)$, i.e.

$$
2 \Delta=\sum_{k, \ell \geq 0}\left(\left(\begin{array}{c}
k+\ell \\
k
\end{array}\right) Q_{k+\ell}-Q_{k} Q_{\ell}\right) \frac{\partial^{2}}{\partial Q_{k+1} \partial Q_{\ell+1}}-\sum_{k \geq 0} Q_{k} \frac{\partial}{\partial Q_{k+2}} .
$$

The operator $\Delta$ acts on $\mathcal{R}$. This action does not restrict to $\mathbb{Q}\left[Q_{2}, Q_{3}, \ldots\right]$, which is isomorphic to $\Lambda^{*}$, as, e.g., $\Delta\left(Q_{3}\right)=-\frac{1}{2} Q_{1}$. However, $\Delta^{*}$, defined by $\operatorname{pr} \Delta \iota$, acts on $\Lambda^{*}$. 
In the following (antisymmetric) table the entry in the row of operator $A$ and column of operator $B$ denotes the commutator $[A, B]$; for proofs see [Zag16, Lemma 3].

\begin{tabular}{c|ccccc} 
& $\Delta$ & $\boldsymbol{\partial}$ & $E$ & $Q_{1}$ & $Q_{2}$ \\
\hline$\Delta$ & 0 & 0 & $2 \Delta$ & 0 & $E-Q_{1} \boldsymbol{\partial}-\frac{1}{2}$ \\
$\boldsymbol{\partial}$ & 0 & 0 & $\boldsymbol{\partial}$ & 1 & $Q_{1}$ \\
$E$ & $-2 \Delta$ & $-\boldsymbol{\partial}$ & 0 & $Q_{1}$ & $2 Q_{2}$ \\
$Q_{1}$ & 0 & -1 & $-Q_{1}$ & 0 & 0 \\
$Q_{2}$ & $-E+Q_{1} \boldsymbol{\partial}+\frac{1}{2}$ & $-Q_{1}$ & $-2 Q_{2}$ & 0 & 0
\end{tabular}

Let $\widehat{Q}_{2}:=Q_{2}-\frac{1}{2} Q_{1}^{2}$ and $\widehat{E}:=E-Q_{1} \partial-\frac{1}{2}$. In terms of these operators, the above table simplifies as follows.

\begin{tabular}{c|ccccc} 
& $\Delta$ & $\partial$ & $\widehat{E}$ & $Q_{1}$ & $\widehat{Q}_{2}$ \\
\hline$\Delta$ & 0 & 0 & $2 \Delta$ & 0 & $\widehat{E}$ \\
$\partial$ & 0 & 0 & 0 & 1 & 0 \\
$\widehat{E}$ & $-2 \Delta$ & 0 & 0 & 0 & $2 \widehat{Q}_{2}$ \\
$Q_{1}$ & 0 & -1 & 0 & 0 & 0 \\
$\widehat{Q}_{2}$ & $-\widehat{E}$ & 0 & $-2 \widehat{Q}_{2}$ & 0 & 0
\end{tabular}

Hence, we have proven the following result.

Proposition 2.2.1. The operators $\left(\widehat{Q}_{2}, \widehat{E}, \Delta\right)$ form an $\mathfrak{s l}_{2}$-triple.

For later reference we compute $\left[\Delta, Q_{2}^{n}\right]$. This could be done inductively by noting that $\left[\Delta, Q_{2}^{n}\right]=Q_{2}^{n-1}\left[\Delta, Q_{2}\right]+\left[\Delta, Q_{2}^{n-1}\right] Q_{2}$ and using the commutation relations in above table. The proof below is a direct computation from the definition of $\Delta$.

Lemma 2.2.2. For all $n \in \mathbb{N}$ the following relation holds

$$
\left[\Delta, Q_{2}^{n}\right]=-\frac{n(n-1)}{2} Q_{1}^{2} Q_{2}^{n-2}-n Q_{1} Q_{2}^{n-1} \boldsymbol{\partial}+n Q_{2}^{n-1}\left(E+n-\frac{3}{2}\right) .
$$

Proof. Let $f \in \mathbb{Q}\left[Q_{1}, Q_{2}\right], g \in \mathscr{R}$ and $n \in \mathbb{N}$. Then

$$
\begin{aligned}
& \Delta(f g)=\Delta(f) g+\frac{\partial f}{\partial Q_{2}}\left(E g-Q_{1} \partial g\right)+f \Delta(g), \\
& \Delta\left(Q_{2}^{n}\right)=n\left(n-\frac{3}{2}\right) Q_{2}^{n-1}-\frac{n(n-1)}{2} Q_{2}^{n-2} Q_{1}^{2} .
\end{aligned}
$$

By (2.5) and (2.6) we find

$$
\begin{aligned}
\Delta\left(Q_{2}^{n} g\right)=\left(n\left(n-\frac{3}{2}\right) Q_{2}^{n-1}\right. & \left.-\frac{1}{2} n(n-1) Q_{1}^{2} Q_{2}^{n-2}\right) g+ \\
& +n Q_{2}^{n-1}\left(E g-Q_{1} \partial g\right)+Q_{2}^{n} \Delta(g) .
\end{aligned}
$$


Now, we turn our attention to the differential operators on quasimodular forms. Recall $P, Q$ and $R$ are Ramanujan's notation for the Eisenstein series of weight 2, 4 and 6, respectively; see Example 1.2.5. In the context of the Bloch-Okounkov theorem, it is more natural to adapt the $\mathfrak{s l}_{2}$-triple of operators acting on quasimodular forms in the following way. Let $\widehat{D}:=D-\frac{P}{24}$, so that for all $f \in \Lambda^{*}$ one has $\left\langle Q_{2} f\right\rangle_{q}=\widehat{D}\langle f\rangle_{q}$. Moreover, $\widehat{D}$ has the property that it increases the depth of a quasimodular form by 1 , in contrast to $D$ for which $D(1)=0$ does not have depth 1 :

Lemma 2.2.3. Let $f \in \widetilde{M}$ be of depth $r$. Then $\widehat{D} f$ is of depth $r+1$.

Proof. Consider a monomial $P^{a} Q^{b} R^{c}$ with $a, b, c \in \mathbb{Z}_{\geq 0}$. By the Ramanujan identities (1.8) we find

$$
D\left(P^{a} Q^{b} R^{c}\right)=\left(\frac{a}{12}+\frac{b}{3}+\frac{c}{2}\right) P^{a+1} Q^{b} R^{c}+O\left(P^{a}\right),
$$

where $O\left(P^{a}\right)$ denotes a quasimodular form of depth at most $a$. The lemma follows by noting that $\frac{a}{12}+\frac{b}{3}+\frac{c}{2}-\frac{1}{24}$ is non-zero for $a, b, c \in \mathbb{Z}$.

Two important ideals in $\Lambda^{*}$ are $I=\operatorname{ker}\langle\cdot\rangle_{q} \cap \Lambda^{*}$ and $\left(Q_{2}\right)=Q_{2} \Lambda^{*}$, related by:

Corollary 2.2.4. Let $f \in \Lambda^{*}$. Then $f \in I$ if and only if $Q_{2} f \in I$.

Proof. If $\langle f\rangle_{q}=0$, then $\left\langle Q_{2} f\right\rangle_{q}=\hat{D}(0)=0$. If $\langle f\rangle_{q} \neq 0$, then $\langle f\rangle_{q}$ is quasimodular of some depth $r$ and $\left\langle Q_{2} f\right\rangle_{q}$ is of some depth $r+1$, in particular non-zero.

Moreover, letting $\widehat{W}=W-\frac{1}{2}$, the triple $(\widehat{D}, \widehat{W}, \mathfrak{d})$ forms an $\mathfrak{s l}_{2}$-triple as well. With respect to these operators the $q$-bracket becomes $\mathfrak{s l}_{2}$-equivariant. The following proposition is a detailed version of Proposition 2.1.1:

Proposition 2.2.5 (The $\mathfrak{s l}_{2}$-equivariant Bloch-Okounkov theorem). The mapping $\langle\cdot\rangle_{q}: \mathcal{R} \rightarrow \widetilde{M}$ is $\mathfrak{s l}_{2}$-equivariant with respect to the $\mathfrak{s l}_{2}$-triple $\left(\widehat{Q}_{2}, \widehat{E}, \Delta\right)$ on $\mathcal{R}$ and the $\mathfrak{s l}_{2}$-triple $(\widehat{D}, \widehat{W}, \mathfrak{d})$ on $\widetilde{M}$, i.e. for all $f \in \mathcal{R}$ one has

$$
\widehat{D}\langle f\rangle_{q}=\left\langle\widehat{Q}_{2} f\right\rangle_{q}, \quad \widehat{W}\langle f\rangle_{q}=\langle\widehat{E} f\rangle_{q}, \quad \mathfrak{d}\langle f\rangle_{q}=\langle\Delta f\rangle_{q} .
$$

Proof. This follows directly from [Zag16, Equation (37)] and the fact that for all $f \in \mathcal{R}$ one has $\left\langle Q_{1} f\right\rangle_{q}=0$. 


\subsection{Describing the space of shifted symmetric harmonic polynomials}

In this section we study the kernel of $\Delta$. As $\left[\Delta, Q_{1}\right]=0$, we restrict ourselves without loss of generality to $\Lambda^{*}$ instead of $\mathcal{R}$. Recall that $\Delta^{*}=\operatorname{pr} \Delta \iota$ acts on $\Lambda^{*}$.

Definition 2.3.1. Let

$$
\mathcal{H}=\left\{f \in \Lambda^{*} \mid \Delta \iota f \in Q_{1} \mathcal{R}\right\}=\operatorname{ker} \Delta^{*},
$$

be the space of shifted symmetric harmonic polynomials.

Proposition 2.3.2. We have a direct sum decomposition

$$
\Lambda^{*}=\mathcal{H} \oplus\left(Q_{2}\right) \quad\left(\left(Q_{2}\right)=Q_{2} \Lambda^{*}\right) .
$$

Proof. First we show that $\mathcal{H} \cap\left(Q_{2}\right)$ is trivial. Let $f$ be an element of this intersection and write $f=Q_{2}^{n} f^{\prime}$ with $n \geq 1, f^{\prime} \in \Lambda^{*}$ and $f^{\prime} \notin\left(Q_{2}\right)$. Then

$$
\Delta^{*}(f)=Q_{2}^{n-1}\left(n\left(n+k-\frac{3}{2}\right) f^{\prime}+Q_{2} \Delta^{*} f^{\prime}\right)
$$

by Lemma 2.2.2. As $f^{\prime}$ is not divisible by $Q_{2}$, it follows that $\Delta^{*}(f)=0$ precisely if $f^{\prime}=0$. Hence, $f=0$ as desired.

In particular, the linear map $T: \Lambda^{*} \rightarrow \Lambda^{*}$, given by $f \mapsto \Delta^{*}\left(Q_{2} f\right)$, is injective. Since $T$ also preserves the degree and each graded component $\Lambda_{n}^{*}$ of $\Lambda^{*}$ is finitedimensional, $T$ is in fact an isomorphism. Hence, given $f \in \Lambda^{*}$ let $g \in \Lambda^{*}$ be such that $T(g)=\Delta^{*}(f) \in \Lambda^{*}$. Let $h=f-Q_{2} g$. As $f=Q_{2} g+h$, it suffices to show that $h \in \mathcal{H}$. That holds true because $\Delta^{*}(h)=\Delta^{*}(f)-\Delta^{*}\left(Q_{2} g\right)=0$.

Proposition 2.3.2 implies Theorem 2.1.3 and the following corollary. Recall $p(n)$ denotes the number of partitions of $n$.

Corollary 2.3.3. The dimension of $\mathcal{H}_{n}$ equals the number of partitions of $n$ into at least 3 parts, i.e.

$$
\operatorname{dim} \mathcal{H}_{n}=p(n)-p(n-1)-p(n-2)+p(n-3) .
$$

Proof. Observe that $\operatorname{dim} \Lambda_{n}^{*}$ equals the number of partitions of $n$ in at least 2 parts. Hence, $\operatorname{dim} \Lambda_{n}^{*}=p(n)-p(n-1)$ and the Corollary follows from Proposition 2.3.2.

Proof of Theorem 2.1.6. Suppose $\langle f\rangle_{q}$ is modular. Then $\langle\Delta f\rangle_{q}=\mathfrak{d}\langle f\rangle_{q}=0$. Write $f=\sum_{r \geq 0} h_{r} Q_{2}^{r}$ as in Theorem 2.1.3. Then by Lemma 2.2.2 it follows that $\Delta^{*} f=$ $\sum_{r \geq 0} r\left(n-r-\frac{3}{2}\right) Q_{2}^{r-1} h_{r}$. Hence,

$$
\sum_{r>0} r\left(n-r-\frac{3}{2}\right) \widehat{D}^{r-1}\left\langle h_{r}\right\rangle_{q}=0
$$


As $\left\langle h_{r}\right\rangle_{q}$ is modular, either it is equal to 0 or it has depth 0 . Suppose the maximum $m$ of all $r \geq 1$ such that $\left\langle h_{r}\right\rangle_{q}$ is non-zero exists. Then, by Lemma 2.2.3 it follows that the left-hand side of (2.7) has depth $m-1$, in particular is not equal to 0 . So, $h_{1}, \ldots, h_{n^{\prime}} \in \operatorname{ker}\langle\cdot\rangle_{q}$, which is the second condition.

By Corollary 2.2.4 the second implication implies the third, and the third the first.

Proof of Theorem 2.1.2. Suppose $\langle f\rangle_{q}$ is modular. By Theorem 2.1.6 it follows directly that $f=h+k$ (namely $h=h_{0}$ and $k=f-h$ ) with $h \in \mathcal{H}$ and $k \in I$. The converse of Theorem 2.1.2 follows directly as $\mathfrak{d}\langle h+k\rangle_{q}=\mathfrak{d}\langle h\rangle_{q}=\left\langle\Delta^{*} h\right\rangle_{q}=0$.

Another corollary of Proposition 2.3.2 is the notion of depth of shifted symmetric polynomials which corresponds to the depth of quasimodular forms:

Definition 2.3.4. The space $\Lambda_{k}^{*(\leq p)}$ of shifted symmetric polynomials of depth $\leq p$ is the space of $f \in \Lambda_{k}^{*}$ such that one can write

$$
f=\sum_{r=0}^{p} Q_{2}^{r} h_{r}
$$

with $h_{r} \in \mathcal{H}_{k-2 r}$.

Theorem 2.3.5. If $f \in \Lambda_{k}^{*(\leq p)}$, then $\langle f\rangle_{q} \in \widetilde{M}_{k}^{(\leq p)}$.

Proof. Expanding $f$ as in Definition 2.3.4 we find

$$
\langle f\rangle_{q}=\sum_{k=0}^{p}\left\langle Q_{2}^{k} h_{k}\right\rangle_{q}=\sum_{k=0}^{p} \widehat{D}^{k}\left\langle h_{k}\right\rangle_{q} .
$$

By Lemma 2.2.3, we find that the depth of $\langle f\rangle_{q}$ is at most $p$.

Next, we set up notation to determine the basis of $\mathcal{H}$ given by Theorem 2.1.4. Let $\widetilde{R}=\mathcal{R}\left[Q_{2}^{-1 / 2}\right]$ and $\tilde{\Lambda}=\Lambda^{*}\left[Q_{2}^{-1 / 2}\right]$ be the formal polynomial algebras graded by assigning to $Q_{k}$ weight $k$ (note that the weights are-possibly negative-integers). Extend $\Delta$ to $\tilde{\Lambda}$ and observe that $\Delta(\tilde{\Lambda}) \subset \tilde{\Lambda}$. Also extend $\mathcal{H}$ by setting

$$
\widetilde{\mathcal{H}}=\left\{f \in \tilde{\Lambda} \mid \Delta \iota f \in Q_{1} \widetilde{\mathscr{R}}\right\} .
$$

Definition 2.3.6. Define the partition-Kelvin transform $K: \tilde{\Lambda}_{n} \rightarrow \tilde{\Lambda}_{3-n}$ by

$$
K(f)=Q_{2}^{3 / 2-n} f .
$$


Note that $K$ is an involution. Moreover, $f$ is harmonic if and only if $K(f)$ is harmonic, which follows directly from the computation

$$
\Delta K(f)=Q_{2}^{3 / 2-n} \Delta f-\left(\frac{3}{2}-n\right) Q_{1} Q_{2}^{\frac{1}{2}-n} \partial f-\frac{1}{2}\left(\frac{3}{2}-n\right)\left(\frac{1}{2}-n\right) Q_{1}^{2} Q_{2}^{-\frac{1}{2}-n} f .
$$

Example 2.3.7. As $K(1)=Q_{2}^{3 / 2}$, it follows that $Q_{2}^{3 / 2} \in \widetilde{\mathcal{H}}$.

Definition 2.3.8. Given $i \in \mathbb{Z}_{\geq 0}^{n}$, let

$$
|\boldsymbol{i}|=i_{1}+i_{2}+\ldots+i_{n}, \quad \partial_{\boldsymbol{i}}=\frac{\partial^{n}}{\partial Q_{i_{1}+1} \partial Q_{i_{2}+1} \cdots \partial Q_{i_{n}+1}} .
$$

Define the $n$th order differential operators $\mathscr{D}_{n}$ on $\widetilde{\mathscr{R}}$ by

$$
\mathscr{D}_{n}=\sum_{\boldsymbol{i} \in \mathbb{Z}_{\geq 0}^{n}}\left(\begin{array}{c}
|\boldsymbol{i}| \\
i_{1}, i_{2}, \ldots, i_{n}
\end{array}\right) Q_{|\boldsymbol{i}|} \partial_{\boldsymbol{i}},
$$

where the coefficient is a multinomial coefficient.

This definition generalises the operators $\boldsymbol{\partial}$ and $\mathscr{D}$ to higher weights: $\mathscr{D}_{1}=\boldsymbol{\partial}$, $\mathscr{D}_{2}=\mathscr{D}$ and $\mathscr{D}_{n}$ reduces the weight by $n$.

Lemma 2.3.9. The operators $\left\{\mathscr{D}_{n}\right\}_{n \in \mathbb{N}}$ commute pairwise.

Proof. Set $I=|\boldsymbol{i}|$ and $J=|\boldsymbol{j}|$. Let $\boldsymbol{a}^{\hat{k}}=\left(a_{1}, \ldots, a_{k-1}, a_{k+1}, \ldots, a_{n}\right)$. Then

$$
\begin{aligned}
& {\left[\left(\begin{array}{c}
I \\
i_{1}, i_{2}, \ldots, i_{n}
\end{array}\right) Q_{I} \partial_{\boldsymbol{i}},\left(\begin{array}{c}
J \\
j_{1}, j_{2}, \ldots, j_{m}
\end{array}\right) Q_{J} \partial_{\boldsymbol{j}}\right] } \\
= & \sum_{k=1}^{n} \delta_{i_{k}, J-1} J\left(\begin{array}{c}
I \\
i_{1}, i_{2}, \ldots, \hat{i_{k}}, \ldots, i_{n}, j_{1}, j_{2}, \ldots, j_{m}
\end{array}\right) Q_{I} \partial_{\boldsymbol{i}^{\hat{k}}} \partial_{\boldsymbol{j}}+ \\
& -\sum_{l=1}^{m} \delta_{j_{l}, I-1} I\left(\begin{array}{c}
J \\
i_{1}, i_{2}, \ldots, i_{n}, j_{1}, j_{2}, \ldots, \hat{j}_{l}, \ldots, j_{m}
\end{array}\right) Q_{J} \partial_{\boldsymbol{i}} \partial_{\hat{j}^{\hat{\imath}}} .
\end{aligned}
$$

Hence, $\left[\mathscr{D}_{n}, \mathscr{D}_{m}\right]$ is a linear combination of terms of the form $Q_{|\boldsymbol{a}|+1} \frac{\partial^{n+m-1}}{\partial Q_{a}}$, where $\boldsymbol{a} \in \mathbb{Z}_{>0}^{n+m-1}$. We collect all terms for different vectors $\boldsymbol{a}$ which consists of the same parts (i.e. we group all vectors $\boldsymbol{a}$ which correspond to the same partition). Then, the 
coefficient of such a term equals

$$
\begin{gathered}
\sum_{k=1}^{n} \sum_{\sigma \in \mathfrak{S}_{m+n-1}}\left(a_{\sigma(1)}+\ldots+a_{\sigma(m)}\right)\left(\begin{array}{c}
|\boldsymbol{a}|+1 \\
a_{1}, a_{2}, \ldots, a_{n+m-1}
\end{array}\right)+ \\
\quad-\sum_{l=1}^{m} \sum_{\sigma \in \mathfrak{S}_{m+n-1}}\left(a_{\sigma(1)}+\ldots+a_{\sigma(n)}\right)\left(\begin{array}{c}
|\boldsymbol{a}|+1 \\
a_{1}, a_{2}, \ldots, a_{n+m-1}
\end{array}\right) \\
=(m n-m n) \sum_{\sigma \in \mathfrak{S}_{m+n-1}} a_{\sigma(1)}\left(\begin{array}{c}
|\boldsymbol{a}|+1 \\
a_{1}, a_{2}, \ldots, a_{n+m-1}
\end{array}\right)=0 .
\end{gathered}
$$

Hence, $\left[\mathscr{D}_{n}, \mathscr{D}_{m}\right]=0$.

It does not hold true that $\left[\mathscr{D}_{n}, Q_{1}\right]=0$ for all $n \in \mathbb{N}$. Therefore, we introduce the following operators:

Definition 2.3.10. Let

$$
\Delta_{n}=\sum_{i=0}^{n}(-1)^{i}\left(\begin{array}{l}
n \\
i
\end{array}\right) \mathscr{D}_{n-i} \partial^{i}
$$

For $\lambda \in \mathscr{P}$ let

$$
\Delta_{\lambda}=\left(\begin{array}{c}
|\lambda| \\
\lambda_{1}, \ldots, \lambda_{\ell(\lambda)}
\end{array}\right) \prod_{i=1}^{\infty} \Delta_{\lambda_{i}} .
$$

(Note that $\Delta_{0}=\mathscr{D}_{0}=1$, so this is in fact a finite product.)

Remark 2.3.11. By Möbius inversion

$$
\mathscr{D}_{n}=\sum_{i=0}^{n}\left(\begin{array}{c}
n \\
i
\end{array}\right) \Delta_{n-i} \partial^{i} .
$$

The first three operators are given by

$$
\Delta_{0}=1, \quad \Delta_{1}=0, \quad \Delta_{2}=\mathscr{D}-\partial^{2}=2 \Delta .
$$

Proposition 2.3.12. The operators $\Delta_{\lambda}$ satisfy the following properties: for all partitions $\lambda, \lambda^{\prime}$

(a) the order of $\Delta_{|\lambda|}$ is $|\lambda|$;

(b) $\left[\Delta_{\lambda}, \Delta_{\lambda^{\prime}}\right]=0$;

(c) $\left[\Delta_{\lambda}, Q_{1}\right]=0$. 
Proof. Property (a) follows by construction and property (b) is a direct consequence of Lemma 2.3.9. For property (c), let $f \in \tilde{\Lambda}$ be given. Then

$$
\begin{aligned}
\Delta_{n}\left(Q_{1} f\right) & =\sum_{i=0}^{n}(-1)^{i}\left(\begin{array}{c}
n \\
i
\end{array}\right) \mathscr{D}_{n-i} \partial^{i}\left(Q_{1} f\right) \\
& =\sum_{i=0}^{n}(-1)^{i}\left(\begin{array}{c}
n \\
i
\end{array}\right)\left((n-i) \mathscr{D}_{n-i-1} \partial^{i} f+Q_{1} \mathscr{D}_{n-i} \partial^{i} f+i \mathscr{D}_{n-i} \partial^{i-1} f\right) \\
& =Q_{1} \Delta_{n}(f)+\sum_{i=0}^{n}(-1)^{i}\left(\begin{array}{c}
n \\
i
\end{array}\right)\left((n-i) \mathscr{D}_{n-i-1} \partial^{i} f+i \mathscr{D}_{n-i} \partial^{i-1} f\right) .
\end{aligned}
$$

Observe that by the identity

$$
(n-i)\left(\begin{array}{c}
n \\
i
\end{array}\right)=(i+1)\left(\begin{array}{c}
n \\
i+1
\end{array}\right)
$$

the sum in the last line is a telescoping sum, equal to zero. Hence $\Delta_{n}\left(Q_{1} f\right)=$ $Q_{1} \Delta_{n}(f)$ as desired.

In particular, above proposition yields $\left[\Delta_{\lambda}, \Delta\right]=0$ and $\left[\Delta_{\lambda}, \mathrm{pr}\right]=0$.

Denote by $(x)_{n}^{-}$the falling factorial power $(x)_{n}^{-}=\prod_{i=0}^{n-1}(x-i)$ and for $\lambda \in \mathscr{P}_{n}$ define $Q_{\lambda}=\prod_{i=1}^{\infty} Q_{\lambda_{i}}$. Let

$$
h_{\lambda}=\operatorname{pr} K \Delta_{\lambda} K(1) .
$$

Observe that $h_{\lambda}$ is harmonic, as pr $\Delta$ commutes with pr and $\Delta_{\lambda}$.

Remark 2.3.13. In Theorem 7.3.4 we find a different expression for $h_{\lambda}$.

Proposition 2.3.14. For all $\lambda \in \mathscr{P}_{n}$ there exists an $f \in \Lambda_{n-2}^{*}$ such that

$$
h_{\lambda}=\left(\frac{3}{2}\right)_{n}^{-} n ! Q_{\lambda}+Q_{2} f .
$$

Proof. Note that the left-hand side is an element of $\Lambda^{*}$ of which the monomials divisible by $Q_{2}^{i}$ correspond precisely to terms in $\Delta_{\lambda}$ involving precisely $n-i$ derivatives of $K(1)$ to $Q_{2}$. Hence, as $\Delta_{\lambda}$ has order $n$ all terms not divisible by $Q_{2}$ correspond to terms in $\Delta_{\lambda}$ which equal $\frac{\partial}{\partial Q_{2}^{n-i}}$ up to a coefficient. There is only one such term in $\Delta_{\lambda}$, with coefficient $\left(\begin{array}{c}|\lambda| \\ \lambda_{1}, \ldots, \lambda_{r}\end{array}\right) \lambda_{1} ! \ldots \lambda_{r} ! Q_{\lambda}$.

Proof of Theorem 2.1.4. Let $\mathcal{B}_{n}=\left\{h_{\lambda} \mid \lambda \in \mathscr{P}_{n}\right.$ all parts are $\left.\geq 3\right\}$. First of all, observe that by Corollary 2.3.3 the number of elements in $\mathcal{B}_{n}$ is precisely the dimension of $\mathcal{H}_{n}$. Moreover, the weight of an element in $\mathcal{B}_{n}$ equals $|\lambda|=n$. By Proposition 2.3.14 it follows that the elements of $\mathcal{B}_{n}$ are linearly independent harmonic shifted symmetric polynomials. 
We embed $\mathcal{R}$ in $\operatorname{End}(\mathcal{R})$ via the algebra morphism $\vee$ uniquely determined by $Q_{i} \mapsto \Delta_{i}$. Furthermore, for $f \in \Lambda^{*}$, we let $f^{\vee}$ be the operator where every occurrence of $Q_{i}$ in $f$ is replaced by $\Delta_{i}$. We get the following unusual identity:

Corollary 2.3.15. If $h \in \mathcal{H}_{n}$, then

$$
h=\frac{\operatorname{pr} K h^{\vee} K(1)}{n !\left(\frac{3}{2}\right)_{n}^{-}} .
$$

Proof. By Proposition 2.3.14 we know that the statement holds true up to adding $Q_{2} f$ to the right-hand side for some $f \in \Lambda_{n-2}^{*}$. However, as both sides of (2.9) are harmonic and the shifted symmetric polynomial $Q_{2} f$ is harmonic precisely if $f=0$ by Proposition 2.3.2, it follows that $f=0$ and (2.9) holds true. 
CHAPTER 3

\section{Quasimodularity of Hurwitz numbers over elliptic} curves

\subsection{Introduction}

In Example 1.5.1 we saw that the simple Hurwitz numbers of genus $g$ and degree $d$ over a curve of genus 1 are given by

$$
\sum_{|\lambda|=d} Q_{3}(\lambda)^{2 g-2}
$$

In this chapter we define Hurwitz numbers as well as triply mixed Hurwitz numbers, a generalised version of Hurwitz numbers originating from tropical geometry. The main technical result is that the above expression still holds, after replacing $Q_{3}^{2 g-2}$ by a different explicit shifted symmetric function.

Proposition 3.1.1. For every triple mixed Hurwitz number $h$ of degree d over a curve of genus 1 there exists an $f \in \Lambda^{*}$ such that

$$
h=\sum_{\lambda \vdash d} f(\lambda),
$$

where the sum is over all partitions of size $d$.

The connection between Hurwitz numbers and shifted symmetric functions is made via central characters in the representation theory of the symmetric group, which on the one hand occur naturally when counting Hurwitz numbers and on the other hand are shifted symmetric functions themselves. By the Bloch-Okounkov theorem, if follows directly from Proposition 3.1.1 that the corresponding generating series of triply mixed Hurwitz numbers are quasimodular forms, which is Theorem B.

This chapter is based on [HIL19], joint work with Marvin A. Hahn and Felix Leid. In that paper we also provide refined quasimodularity results, as well as results on topological recursion. We leave these results out of this thesis, as they go beyond the topic of this chapter. 


\subsection{Triply mixed Hurwitz numbers}

We define classical Hurwitz numbers via a counting problem in the symmetric group. This naturally leads to the generalisation to triply mixed Hurwitz numbers.

Given a permutation $\sigma$ in the symmetric group $\mathfrak{S}_{d}$, denote by $\operatorname{ptn}(\sigma)$ the partition which corresponds to the cycle type of $\sigma$.

Definition 3.2.1. Let $g^{\prime}, g \geq 0$ be non-negative integers, $d$ a positive integer and $\mu=\left(\mu^{1}, \ldots, \mu^{n}\right)$ a tuple of partitions of $d$. In case

$$
2 g^{\prime}-2=d(2 g-2)+\sum_{j=1}^{n}\left(\left|\mu^{j}\right|-\ell\left(\mu^{j}\right)\right),
$$

we call $\left(\sigma_{1}, \ldots, \sigma_{n}, \alpha_{1}, \beta_{1}, \ldots, \alpha_{g}, \beta_{g}\right)$ a factorization of type $\left(g, g^{\prime}, d, \mu\right)$ if the following conditions are satisfied for all $i$ :

(1) $\sigma_{i}, \alpha_{i}, \beta_{i} \in \mathfrak{S}_{d}$;

(2) $\sigma_{1} \cdots \sigma_{n}=\left[\alpha_{1}, \beta_{1}\right] \cdots\left[\alpha_{g}, \beta_{g}\right]$

(3) $\operatorname{ptn}\left(\sigma_{i}\right)=\mu^{i}$.

If additionally we have

(C) $\left\langle\sigma_{1}, \ldots, \sigma_{n}, \alpha_{1}, \beta_{1}, \ldots, \alpha_{g}, \beta_{g}\right\rangle$ acts transitively on the set $\{1,2, \ldots, d\}$

we call the factorisation connected. Denote by $\mathcal{F}^{\bullet}\left(g, g^{\prime}, d, \mu\right)$ and $\mathcal{F}\left(g, g^{\prime}, d, \mu\right)$ the sets of factorisations and connected factorisations of type $\left(g, g^{\prime}, d, \mu\right)$, respectively. The Hurwitz numbers and connected Hurwitz numbers are defined by

$$
\begin{aligned}
h_{g}^{g^{\prime}, d ; \bullet}\left(\mu^{1}, \ldots, \mu^{n}\right) & =\frac{1}{d !}\left|\mathcal{F}^{\bullet}\left(g, g^{\prime}, d, \mu\right)\right|, \\
h_{g}^{g^{\prime}, d}\left(\mu^{1}, \ldots, \mu^{n}\right) & =\frac{1}{d !}\left|\mathcal{F}\left(g, g^{\prime}, d, \mu\right)\right| .
\end{aligned}
$$

Remark 3.2.2. Hurwitz numbers were first defined as an enumerative problem counting ramified morphisms between Riemann surfaces: For a fixed compact Riemann surface $S$ of genus $g$, Hurwitz numbers count holomorphic maps $\pi: S^{\prime} \rightarrow S$ (up to isomorphism) of degree $d$, where $S^{\prime}$ is a compact Riemann surface of genus $g^{\prime}$, such that

- $\pi$ has ramification profile $\mu^{1}, \ldots, \mu^{n}$ over $n$ arbitrary, but fixed points on $S$;

- each map is weighted by $|\operatorname{Aut}(\pi)|^{-1}$. 
The connection to our definition, which is due to Hurwitz, is made by considering the monodromy representations for the holomorphic maps involved (see [Hur1891, Hur 1901]). The condition (3.1) is the Riemann-Hurwitz formula, a necessary condition for the existence for the holomorphic maps $\pi$ counted by the Hurwitz numbers. Condition $\mathrm{C}$ is exactly the condition for the Riemann surface $S^{\prime}$ to be connected. For more details, see, e.g., [CM16].

Often condition (3.1) is relaxed by allowing $\mu$ to be extended by an arbitrary number of simple partitions, i.e., partitions of the form $(2,1,1, \ldots, 1)$. Triply mixed Hurwitz numbers are Hurwitz numbers extended in this way, with an additional requirement on the transpositions (corresponding to these simple partitions).

Definition 3.2.3. Let $g^{\prime}, g \geq 0$ be non-negative integers, $d$ a positive integer and $\mu=\left(\mu^{1}, \ldots, \mu^{n}\right)$ a tuple of partitions (not necessarily of the same integers). Furthermore, let

$$
b=b\left(g, g^{\prime}, \mu\right)=2 g^{\prime}-2-d(2 g-2)+\sum_{i}\left(\ell\left(\mu^{i}\right)-\left|\mu^{i}\right|\right) .
$$

For non-negative integers $k, l, m$ such that $k+l+m=b$, we define a triply mixed factorisation of type $(g, d, \mu, k, l, m)$ to be a tuple

$$
\left(\sigma_{1}, \ldots, \sigma_{n}, \tau_{1}, \ldots, \tau_{b}, \alpha_{1}, \beta_{1}, \ldots, \alpha_{g}, \beta_{g}\right) \in \mathfrak{S}_{d}^{n+b+2 g}
$$

such that for all $i$

(1) the $\tau_{i}$ are transpositions;

(2) $\sigma_{1} \cdots \sigma_{n} \tau_{1} \cdots \tau_{b}=\left[\alpha_{1}, \beta_{1}\right] \cdots\left[\alpha_{g}, \beta_{g}\right]$;

(3) $\operatorname{ptn}\left(\sigma_{i}\right)$ and $\mu^{i}$ differ only in the amount of parts equal to 1 ;

(4) for $\tau_{i}=\left(s_{i} t_{i}\right)$ with $s_{i}<t_{i}$, we have

- $t_{i} \leq t_{i+1}$ for $i=k+1, \ldots, k+l-1$,

- $t_{i}<t_{i+1}$ for $i=k+l+1, \ldots, k+l+m-1$.

If in addition, we have

(C) $\left\langle\sigma_{1}, \ldots, \sigma_{n}, \tau_{1}, \ldots, \tau_{b}, \alpha, \beta\right\rangle$ acts transitively on $\{1, \ldots, d\}$,

we call the factorisation connected. We denote by $M^{\bullet}(g, d, \mu, k, l, m)$ the set of triply mixed factorisations of type $(g, d, \mu, k, l, m)$ and by $M(g, d, \mu, k, l, m)$ the set of connected triply mixed factorisations of type $(g, d, \mu, k, l, m)$. Then we define 
the triply mixed Hurwitz numbers and the connected triply mixed Hurwitz numbers, respectively, by

$$
\begin{aligned}
h_{g ; k, l, m}^{g^{\prime}, d \bullet \bullet}(\mu) & =\frac{1}{d !}\left|M^{\bullet}(g, d, \mu, k, l, m)\right|, \\
h_{g ; k, l, m}^{g^{\prime}, d}(\mu) & =\frac{1}{d !}|M(g, d, \mu, k, l, m)| .
\end{aligned}
$$

Remark 3.2.4. By taking $l=m=0$ we recover classical Hurwitz numbers, now extended with additional simple partitions. In case $k=m=0$ we call the corresponding triply mixed Hurwitz numbers monotone Hurwitz numbers and in case $k=l=0$ we call them strictly monotone Hurwitz numbers. Strictly monotone Hurwitz numbers are also called Grothendieck dessins d'enfants Hurwitz numbers, as they are equivalent to an enumeration of Grothendieck dessins d'enfants in tropical geometry, as proved in [ALS16, section 4.3.2]. One way to think about triply mixed Hurwitz numbers is as an interpolations between classical, monotone and strictly monotone Hurwitz numbers.

\subsection{Central characters of the symmetric group are shifted symmetric}

We aim to express triply mixed Hurwitz numbers in terms of shifted symmetric functions. This will be done via the central characters $f_{\nu}$ in the representation theory of the symmetric group. In this section we define these central characters, as well as the Jucys-Murphy elements occurring in these central characters.

Let $d \geq 0$ and $\mathcal{Z}_{d}$ be the center of the group algebra $\mathbb{C}\left[\mathfrak{S}_{d}\right]$ of the symmetric group. Given a partition $\lambda$, denote by $C_{\lambda}$ the sum of all elements in $\mathfrak{S}_{|\lambda|}$ of cycle type $\lambda$. Let $\left(\rho_{\lambda}, V_{\lambda}\right)$ be the irreducible representation of $\mathfrak{S}_{|\lambda|}$ corresponding to $\lambda$. Note that a basis of $\mathcal{Z}_{d}$ is given by $C_{\lambda}$ for all partitions $\lambda$ of $d$.

Definition 3.3.1. Given $z \in \mathcal{Z}_{d}$, the central character $\omega^{\lambda}(z)$ is the constant by which $\rho_{\lambda}(z)$ acts on $V_{\lambda}$ (recall that by Schur's lemma $\rho_{\lambda}(z)$ acts by a constant).

For example, the central character of the conjugacy class sums $C_{\nu}$ is given by

$$
f_{\nu}(\lambda):=\omega^{\lambda}\left(C_{\nu}\right)=\left|C_{\nu}\right| \frac{\chi^{\lambda}(\nu)}{\operatorname{dim} \lambda}
$$

where (by abuse of notation) $\left|C_{\nu}\right|$ denotes the size of the conjugacy class of elements of cycle type $\nu$ in $\mathfrak{S}_{|\nu|}, \chi^{\lambda}$ denotes the character of $\rho_{\lambda}$ and $\operatorname{dim} \lambda$ the dimension of $\rho_{\lambda}$. We extend 3.3 to the case when $|\nu| \neq|\lambda|$ by

$$
f_{\nu}(\lambda):=\left(\begin{array}{l}
|\lambda| \\
|\nu|
\end{array}\right)\left|C_{\nu}\right| \frac{\chi^{\lambda}(\bar{\nu})}{\operatorname{dim} \lambda}
$$


where $f_{\nu}(\lambda)=0$ whenever $|\lambda|<|\nu|$ and $\bar{\nu}$ denotes the partition of size $|\lambda|$ differing from $\nu$ only in the amount of parts equal to 1 . Observe that if $\nu$ is a partition without parts equal to 1 the quantity $\left(\begin{array}{l}|\lambda| \\ |\nu|\end{array}\right)\left|C_{\nu}\right|$ equals the size of the conjugacy class of cycle type $\nu$ in $\mathfrak{S}_{|\lambda|}$ instead of in $\mathfrak{S}_{|\nu|}$. These central characters are in fact shifted symmetric functions, as was proven in [KO94, Theorem 5].

Lemma 3.3.2. The central character $f_{\nu}$ is a shifted symmetric function of mixed weight at most $|\nu|+\ell(\nu)$.

Let $\Xi_{d}=\left(J_{1}, J_{2}, \ldots, J_{d}, 0,0, \ldots\right)$ be the sequence of Jucys-Murphy elements given by $J_{k}=\sum_{i=1}^{k-1}(i k) \in \mathbb{C}\left[\mathfrak{S}_{d}\right]$. Although the Jucys-Murphy elements do not belong to $\mathcal{Z}_{d}$, symmetric polynomials in $\Xi_{d}$ are elements of $\mathcal{Z}_{d}$. More precisely, every element of $\mathcal{Z}_{d}$ can be written as a symmetric polynomial in $\Xi_{d}$ [Juc74, Mur81]. Remarkably, the central character of a symmetric polynomial $f$ evaluated at $\Xi_{d}$ has the following simple expression.

Denote by $\operatorname{cont}_{\lambda}$ the set of all contents of the partition $\lambda$, i.e.,

$$
\operatorname{cont}_{\lambda}=\left\{j-i \mid(i, j) \in Y_{\lambda}\right\} .
$$

Proposition 3.3.3. Let $f$ be a symmetric polynomial of degree $n$. Then

(i) $\omega^{\lambda}\left(f\left(\Xi_{d}\right)\right)=f\left(\right.$ cont $\left._{\lambda}\right)$, where $\lambda$ is a partition of size $d$.

(ii) As a function of $\lambda$, the central character $\omega^{\lambda}\left(f\left(\Xi_{d}\right)\right)$ is a shifted symmetric function of mixed weight at most $3 n$.

(iii) If $f$ is the complete homogeneous symmetric polynomial $h_{n}$ or the elementary symmetric polynomial $e_{n}$ of degree $n$, then

$$
\omega^{\lambda}\left(f\left(\Xi_{d}\right)\right)-\frac{1}{2^{n-1}} Q_{3}(\lambda)^{n}
$$

is shifted symmetric of mixed weight at most $3 n-1$.

Proof. The first statement follows directly from [Juc74].

Next, let $k \geq 0$ and $(x)_{k}=x(x-1) \cdots(x-k+1)$. Then, for the symmetric polynomial $\underline{p}_{k}\left(x_{1}, \ldots, x_{m}\right):=\sum_{i=1}^{m}\left(x_{i}\right)_{k}$ it is known that [KO94, Theorem 4]

$$
k \underline{p}_{k-1}\left(\operatorname{cont}_{\lambda}\right)=\sum_{i=1}^{\infty}\left(\left(\lambda_{i}-i+1\right)_{k}-(-i+1)_{k}\right) \quad(k \geq 1)
$$

where the right-hand side is a shifted symmetric polynomial of mixed degree $\leq k+1$.

Given a symmetric polynomial $g$ of degree $n$ with constant term equal to zero, one can write $g$ as a polynomial in the $\underline{p}_{k}$ for $k \geq 1$. Assign to $\underline{p}_{k}$ weight $k+2$ in 
accordance with (3.5), i.e., $\underline{p}_{k}\left(\operatorname{cont}_{\lambda}\right)$ is a shifted symmetric polynomial of mixed weight $k+2$. Observe that the monomial $\underline{p}_{1}^{n}$ is the unique monomial of degree $n$ containing a non-trivial term of weight $3 n$. Hence, the central character $\omega^{\lambda}\left(f\left(\Xi_{d}\right)\right)$ of $g$ is a shifted symmetric function of mixed weight at most $3 n$ with top-degree part up to a multiplicative constant equal to $Q_{3}(\lambda)^{n}$. Specializing $g$ to $h_{n}$ and $e_{n}$ the result follows by observing that in this case the coefficient of $\underline{p}_{1}^{n}$ equals $2^{1-n}$.

The commutator sum $\mathfrak{K}=\sum_{\alpha, \beta \in \mathfrak{S}_{d}}[\beta, \alpha]$ also is an element of $\mathcal{Z}_{d}$. Its central character equals [CM16, Eq. 9.14]

$$
\omega^{\lambda}(\mathfrak{K})=\left(\frac{d !}{\operatorname{dim} \lambda}\right)^{2} .
$$

\subsection{Quasimodularity of triply mixed Hurwitz numbers}

Fix $g^{\prime} \geq 2$. The generating series

$$
\sum_{d \geq 1} h_{k, 0,0}^{g^{\prime}, d ; \bullet}(\mu) q^{d}
$$

of ordinary Hurwitz numbers with $g=1$ is known to be a quasimodular form. This was observed by Dijkgraaf in the simplest case $(\mu=())$, rigorously proved in that case by Kaneko and Zagier, and follows in full generality from the BlochOkounkov theorem as noted by Eskin and Okounkov, and independently by Ochiai [Dij95, KZ95, EO01, Och01]. In this section we extend this result to the generating series of triply mixed Hurwitz numbers with $g=1$. We begin by expressing triply mixed Hurwitz numbers in terms of shifted symmetric functions.

Let $h_{n}$ be the complete homogeneous symmetric polynomial and $e_{n}$ the elementary symmetric polynomial of degree $n$, respectively. Proposition 3.1.1 in the introduction is implied by the following result.

Proposition 3.4.1. Let $g, g^{\prime} \geq 0$, let $\mu$ be a tuple of partitions and take $k, l, m \geq 0$ with $k+l+m=b$, where $b=b\left(g, g^{\prime}, \mu\right)$ is given by (3.2). Then, we have

$$
h_{g ; k, l, m}^{g^{\prime}, d ; \bullet}(\mu)=\sum_{\lambda \vdash d}\left(\frac{\operatorname{dim} \lambda}{d !}\right)^{2-2 g} f_{\mu}(\lambda) f_{(2)}(\lambda)^{k} h_{l}\left(\operatorname{cont}_{\lambda}\right) e_{m}\left(\operatorname{cont}_{\lambda}\right),
$$

where the sum is over all partitions of size $d$.

Proof. First, we rewrite the triply mixed Hurwitz number in terms of the center $\mathcal{Z}_{d}$ of the group algebra $\mathfrak{S}_{d}$. Given a partition $\lambda$ of $d$ recall the elements $C_{\lambda}$ in $\mathcal{Z}_{d}$ 
are defined as the sum of all permutations of cycle type $\lambda$. In case $\lambda$ is a partition of size smaller than $d$, we extend $\lambda$ with additional parts equal to 1 . Observe that $\left[C_{e}\right] C_{\mu^{1}} \cdots C_{\mu^{n}}$, where $\left[C_{e}\right]$ denotes the coefficient of $C_{e}=e$ in the basis of $\mathcal{Z}_{d}$ given by the $C_{\lambda}$, counts the number of $\sigma_{1}, \ldots, \sigma_{n}$ where the cycle type of $\sigma_{i}$ is $\mu^{i}$ (up to parts equal to 1 ) and for which $\sigma_{1} \cdots \sigma_{n}=e$.

Next, note that

$$
h_{k}\left(\Xi_{d}\right)=\sum_{\substack{2 \leq t_{1} \leq \ldots \leq \leq t_{k} \leq d \\ s_{i}<t_{i}}}\left(s_{1} t_{1}\right) \cdots\left(s_{k} t_{k}\right)
$$

and

$$
e_{k}\left(\Xi_{d}\right)=\sum_{\substack{2 \leq t_{1}<\ldots<t_{k} \leq d \\ s_{i}<t_{i}}}\left(s_{1} t_{1}\right) \cdots\left(s_{k} t_{k}\right) .
$$

Hence, $\left[C_{e}\right] C_{(2)}^{k} h_{l}\left(\Xi_{d}\right) e_{m}\left(\Xi_{d}\right)$ counts the number of transpositions $\tau_{1}, \ldots, \tau_{b}$ satisfying conditions (1) and (4) in the definition of a triply mixed factorisations (Definition 3.2.3). Hence, equivalently a triply mixed Hurwitz number is given by

$$
h_{g ; k, l, m}^{g^{\prime}, d ; \bullet}(\mu)=\frac{1}{d !}\left[C_{e}\right] \mathfrak{K}^{g} C_{\mu^{1}} \cdots C_{\mu^{n}} C_{(2)}^{k} h_{l}\left(\Xi_{d}\right) e_{m}\left(\Xi_{d}\right) .
$$

Note that $\chi^{\lambda}\left(C_{\nu}\right)=\chi^{\lambda}(e) \omega^{\lambda}\left(C_{\nu}\right)$ for all partitions $\nu$ of size $d$. Hence, the Schur orthogonality relation can be written in the unusual form $\sum_{\lambda \vdash d} \chi^{\lambda}(e)^{2} \omega^{\lambda}\left(C_{\nu}\right)=$ $\delta_{e \nu}\left|\mathfrak{S}_{d}\right|$, where $\delta$ denotes the Kronecker delta. Recall $\chi^{\lambda}(e)=\operatorname{dim} \lambda$ and $\left|\mathfrak{S}_{d}\right|=d$ ! . Hence, writing $z \in \mathcal{Z}_{d}$ in the basis given by the $C_{\lambda}$ we find

$$
\left[C_{e}\right] z=\frac{1}{d !} \sum_{\lambda \vdash d}(\operatorname{dim} \lambda)^{2} \omega^{\lambda}(z) .
$$

Therefore,

$$
\begin{aligned}
h_{g ; k, l, m}^{g^{\prime}, d \bullet \bullet}(\mu) & =\sum_{\lambda \vdash d}\left(\frac{\operatorname{dim} \lambda}{d !}\right)^{2} \omega^{\lambda}\left(\mathfrak{K}^{g} C_{\mu^{1}} \cdots C_{\mu^{n}} C_{(2)}^{k} h_{l}\left(\Xi_{d}\right) e_{m}\left(\Xi_{d}\right)\right) \\
& =\sum_{\lambda \vdash d}\left(\frac{\operatorname{dim} \lambda}{d !}\right)^{2-2 g} f_{\mu}(\lambda) f_{(2)}(\lambda)^{k} h_{l}\left(\operatorname{cont}_{\lambda}\right) e_{m}\left(\operatorname{cont}_{\lambda}\right),
\end{aligned}
$$

where in the second equality we used that $\omega^{\lambda}$ is a group homomorphism, as well as (3.6), (3.3) and Proposition 3.3.3.

Definition 3.4.2. Let $t_{\mu}=\prod_{i, j} t_{i, \mu_{j}^{i}}$ be a formal variable. Define the Hurwitz potential by

$$
\mathfrak{H}^{\bullet}=\sum h_{k, l, m}^{g^{\prime}, d \bullet \bullet}(\mu) t_{\mu} \frac{u^{k}}{k !} v^{l} w^{m} q^{d},
$$


where the sum is over all $k, l, m, d, \mu$ for which $h_{k, l, m}^{g^{\prime}, d ; \bullet}(\mu)$ is defined. Note that we set $g=1$. Analogously define the connected Hurwitz potential $\mathfrak{H}$.

Remark 3.4.3. By a standard argument (see e.g. [CM16, Thm. 10.2.1]) the Hurwitz potential and the connected Hurwitz potential are related by

$$
\exp \mathfrak{H}=1+\mathfrak{H}^{\bullet} .
$$

Theorem 3.4.4 (= Theorem B). Let $g^{\prime} \geq 2$. Then

$$
\sum_{d=1}^{\infty} h_{k, l, m}^{g^{\prime}, d}(\mu) q^{d}
$$

is a quasimodular form of mixed weight $\leq 6 g^{\prime}-6+\sum_{i}\left(4 \ell\left(\mu^{i}\right)-2\left|\mu^{i}\right|\right)$. Moreover, for fixed $b=k+l+m$ the top weight parts of

$$
2^{l+m+\delta_{l, 0}+\delta_{m, 0}-2} \sum_{d=1}^{\infty} h_{k, l, m}^{g^{\prime}, d}(\mu) q^{d}
$$

ranging over all $k, l, m \geq 0$ are equal.

Proof. Observe that $f_{\mu}(\lambda) f_{(2)}(\lambda)^{k} h_{l}\left(\operatorname{cont}_{\lambda}\right) e_{m}\left(\operatorname{cont}_{\lambda}\right)$ is shifted symmetric in $\lambda$ of mixed weight at most $\sum_{i}\left(\left|\mu^{i}\right|+\ell\left(\mu_{i}\right)\right)+3(k+l+m)$ by the results in Section 3.3. By (3.2) this weight equals

$$
6 g^{\prime}-6+\sum_{i}\left(4 \ell\left(\mu^{i}\right)-2\left|\mu^{i}\right|\right) .
$$

Observe that evaluating $f_{\mu}, h_{l}$ (cont) or $e_{m}$ (cont) at the empty partition yields 0 unless $\mu$ is empty, $l=0$ or $m=0$, respectively. In other words, using (3.7) to define Hurwitz numbers for $d=0$, one obtains $h_{k, l, m}^{g^{\prime}, 0}(\mu)=1$ if $\mu=()$ and $k=l=m=0$ and 0 else. Hence, Proposition 3.4.1 and Remark 3.4.3 imply that

$$
\mathfrak{H}=\log \left(q^{1 / 24} \eta(\tau)^{-1} \sum\left\langle f_{\mu} f_{(2)}^{k} h_{l}(\text { cont }) e_{m}(\text { cont })\right\rangle_{q} t_{\mu} \frac{u^{k}}{k !} v^{l} w^{m}\right),
$$

where the sum is over all partitions $\mu$ and $k, l, m \geq 0$. The constant $q^{1 / 24} \eta(\tau)^{-1}$ cancels the denominator of the $q$-bracket. By the Bloch-Okounkov theorem the $q$ bracket $\left\langle f_{\mu} f_{(2)}^{k} h_{l} \text { (cont) } e_{m} \text { (cont) }\right\rangle_{q}$ is a quasimodular form of weight at most given by Equation (3.9). Also, for $\mu$ the empty partition and $k=l=m=0$, this $q$-bracket equals 1 . Write $\sum^{\prime}$ for the sum is over all partitions $\mu$ and $k, l, m \geq 0$ excluding this trivial case. Then, all Taylor coefficients of

$$
\mathfrak{H}=-\log \left(q^{-1 / 24} \eta(\tau)\right)+\log \left(1+\sum^{\prime}\left\langle f_{\mu} f_{(2)}^{k} h_{l}(\text { cont }) e_{m}(\text { cont })\right\rangle_{q} t_{\mu} \frac{u^{k}}{k !} v^{l} w^{m}\right),
$$


except the constant coefficient $-\log \left(q^{-1 / 24} \eta(\tau)\right)$, are quasimodular. As this constant term corresponds to $g^{\prime}=1$, quasimodularity of (3.8) follows for all $g^{\prime} \geq 2$. The second part of the statement follows directly from Proposition 3.3.3(iii).

Remark 3.4.5. In fact, the above proofs shows that in case $g^{\prime}=1$ the series in (3.8) equals $-\log \left(q^{-1 / 24} \eta(\tau)\right)$. This is not a quasimodular form, but it is a primitive of a quasimodular form. Namely, its derivative equals up to a constant the Eisenstein series of weight 2. By the Nekrasov-Okounkov formula [NO06] this function also occurs as $\left\langle H_{0}\right\rangle_{q}$ in [CMZ18] (see also the discussion in [Zag16, p. 365]). 



\section{Part II}

\section{A symmetric \\ Bloch-Okounkov theorem}



CHAPTER 4

\section{New quasimodular algebras}

\subsection{Introduction}

The aim of this chapter is to introduce new quasimodular algebras, in the following sense.

Definition 4.1.1. A graded algebra of functions on partitions is called a quasimodular algebra if the $q$-bracket of a homogeneous function $f \in A$ is quasimodular of the same weight as $f$.

Previously, the Bloch-Okounkov algebra $\Lambda^{*}$ and some generalisations to higher levels (see e.g. [EO06, Eng17]), were the only known quasimodular algebras. However, there are many examples of functions on partitions admitting a quasimodular $q$ bracket (and in general not belonging to $\Lambda^{*}$ ) [Zag16, section 9], for example the Möller transformation of functions with quasimodular $q$-bracket (defined by [Zag16, Eqn 45] and recalled in Section 4.12), invariants $\mathcal{A}_{P}$ for every even polynomial defined in terms of the arm- and leg-lengths of a partition and the moment functions

$$
S_{k}(\lambda)=-\frac{B_{k}}{2 k}+\sum_{i=1}^{\infty} \lambda_{i}^{k-1} \quad\left(k \text { even, } B_{k}=k \text { th Bernoulli number }\right)
$$

that also occur in the study of so-called spin Hurwitz numbers in the algebra of supersymmetric polynomials [EOP08] (in that reference, these functions are only evaluated at strict partitions - partitions without repeated parts - and quasimodularity is shown for a correspondingly adapted $q$-bracket).

In this chapter, we prove the stronger result that the algebra $\mathcal{S}$ generated by these moment functions $S_{k}$ is quasimodular. Moreover, besides the pointwise product of functions on partitions we define a second associative product $\odot$, called the induced product as it is inherited from the product of power series. The vector space $\operatorname{Sym}^{\odot}(\mathcal{S})$ generated by the elements in $\mathcal{S}$ under the induced product is strictly

This chapter is based on [121a]. 
bigger than $\mathcal{S}$, is a quasimodular algebra for either of the two products, and has a particularly nice description in terms of functions $T_{k, l}$ depending not only on the parts of a partition, but also on their multiplicities. Here, the multiplicity $r_{m}(\lambda)$ of parts of size $m$ in a partition $\lambda$ is defined as the number of parts of $\lambda$ of size $m$. More precisely, let $\mathcal{F}_{l}$ be the Seki-Bernoulli polynomial of positive integer degree $l$, defined by $\mathcal{F}_{l}(n)=\sum_{i=1}^{n} i^{l-1}$ for all $n \in \mathbb{Z}_{>0}$. Then, $T_{k, l}$ is given by

$$
T_{k, l}(\lambda)=C_{k, l}+\sum_{m=1}^{\infty} m^{k} \mathcal{F}_{l}\left(r_{m}(\lambda)\right) \quad(k \geq 0, l \geq 1, k+l \text { even })
$$

with $C_{k, l}$ a constant equal to $-\frac{B_{k+l}}{2(k+l)}$ if $k=0$ or $l=1$ and 0 else. Let $\mathcal{T}$ be the algebra generated by all these $T_{k, l}$ under the pointwise product.

We show that $\operatorname{Sym}^{\odot}(\mathcal{S})$ and $\mathcal{T}$ are algebras for the pointwise product as well as for the induced product. In fact, the expression of elements of $\operatorname{Sym}^{\odot}(\mathcal{S})$ in terms of the $T_{k, l}$ implies that $\operatorname{Sym}^{\odot}(\mathcal{S})$ is a strict subalgebra of $\mathcal{T}$ (with respect to both products). Our main result is the following:

Theorem 4.1.2 ( $=$ Theorem C). The algebras $\operatorname{Sym}^{\odot}(\mathcal{S})$ and $\mathcal{T}$ are quasimodular algebras with respect to the induced product.

With respect to the pointwise product, these algebras are not quasimodular because of the following subtlety: the $q$-bracket of a homogeneous function $f$ in $\mathcal{T}$ (with respect to the pointwise product) often is of mixed weight (i.e., a linear combination of quasimodular forms of weights bounded by the weight of $f$ ). By making use of the induced product, one can explain these lower weight quasimodular forms, as we do in Section 4.11. For example,

$$
\left\langle T_{0,2}^{2}\right\rangle_{q}=G_{2}^{2}+\frac{5}{6} G_{4}+\frac{1}{6} G_{2}+\frac{1}{288}
$$

is explained by the fact that

$$
T_{0,2}^{2}=T_{0,2} \odot T_{0,2}+\frac{5}{6} T_{0,4}+\frac{1}{6} T_{0,2}+\frac{1}{288}
$$

is a linear combination of elements of $\mathcal{T}$ of different weights with respect to the induced product.

A main theme throughout this chapter is the principle to establish all identities in $\mathbb{Q}^{\mathscr{P}}$ or $\mathcal{T}$ before taking the $q$-bracket, instead of doing these computations in $\mathbb{Q}[[q]]$ or the space of quasimodular forms $\widetilde{M}$. By doing so, we discover the algebraic structure of $\mathcal{T}$. Without having the induced product at one's disposal, for example when studying the shifted symmetric algebra $\Lambda^{*}$, this seems impossible. See the following table for an overview of situations where the principle is applied: 


\begin{tabular}{|c|c|c|}
\hline previous definitio & new definitions and results & $\S \S$ \\
\hline multiplication in $\mathbb{Q}[[q]]$ & induced product $\odot$ on $\mathbb{Q}^{\mathscr{P}}$ & 4.4 \\
\hline q-bracket $: \mathbb{Q}^{\mathscr{P}} \rightarrow \mathbb{Q}[[q]]$ & u-bracket: $\mathbb{Q}^{\mathscr{P}} \rightarrow \mathbb{Q}\left[\left[u_{1}, u_{2}, \ldots\right]\right]$ & 4.4 \\
\hline connected q-bracket: & connected product: & 4.4 \\
\hline $\operatorname{Sym}^{\otimes}\left(\mathbb{Q}^{\mathscr{P}}\right) \rightarrow \mathbb{Q}[[q]]$ & $\operatorname{Sym}^{\otimes}\left(\mathbb{Q}^{\mathscr{P}}\right) \rightarrow \mathbb{Q}^{\mathscr{P}}$ & \\
\hline derivative $q \frac{\mathrm{d}}{\mathrm{d} q}$ on $\mathbb{Q}[[q]]$ & derivative on $\mathbb{Q}^{\mathscr{P}}$ & 4.9 \\
\hline $\mathfrak{s l}_{2}$-action on $\widetilde{M}$ & $\mathfrak{s l}_{2}$-action on $\mathcal{T}$ & 4.10 \\
\hline Rankin-Cohen brackets on $\widetilde{M}$ & Rankin-Cohen brackets on $\mathcal{T}$ & 4.10 \\
\hline $\begin{array}{l}\text { formula for }\left\langle H_{p} f\right\rangle_{q} \text { in } \\
\text { [CMZ18, Eqn 152] }\end{array}$ & formula for $T_{k, l} f$ & 4.11 \\
\hline
\end{tabular}

A further main result of this chapter is the following:

Theorem 4.1.3. The q-bracket is an equivariant mapping $\mathcal{T} \rightarrow \widetilde{M}$ with respect to $\mathfrak{s l}_{2}$-actions by derivations on both spaces.

Motivated by the fact that many functions in invariants of partitions are elements of $\Lambda^{*}$, in Section 4.12 we describe many functions on partitions which are elements of $\mathcal{T}$ or are closely related. Among those are the border strip moments, generalizing the hook-length moments, which are defined in terms of the representation theory of the symmetric group. The corresponding space $\mathcal{X}$ of border strip moments is the image of a space $\mathcal{U}$ under the aforementioned Möller transform $\mathcal{M}$, where $\mathcal{U}$ is generated by the double moment functions $T_{k, l} \in \mathcal{T}$ as well as the odd double moments functions (those for which $k+l$ is odd). The $q$-brackets of these functions are contained in the space $\mathcal{C}$ of so-called combinatorial Eisenstein series, having the space of quasimodular forms as a subspace. Moreover, the space of hooklength moments $\mathcal{H}$ is contained in both $\Lambda^{*}$ and $\mathcal{X}$-this contrasts the situation for $\mathcal{T}$, which by Remark 4.8.6 has a trivial intersection with $\Lambda^{*}$. See the commutative diagram on the right for an overview of the spaces related to $\mathcal{T}$ with their corresponding mappings.

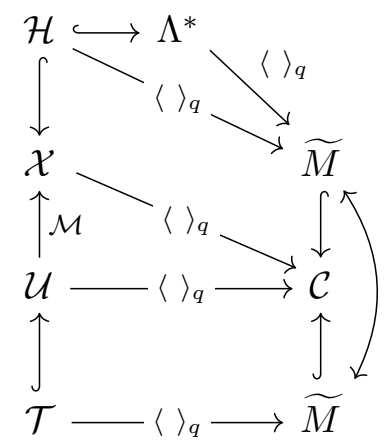

We hope that the results in this chapter-besides advocating the notion of a 'quasimodular algebra' by giving a new example of such an algebra and studying its algebraic structure-may serve as a tool for enumerative geometers trying to show that generating series are quasimodular forms. 


\section{Contents}

The contents of this chapter are as follows. In Section 4.2 we recall notions (known to the experts) related to quasimodular forms, partitions and special families of polynomials. Next, in Sections 4.3 and Section 4.4 we motivate and introduce both the induced and connected product. The quasimodularity of the algebra $\mathcal{S}$ is proven in Section 4.5 and in Section 4.6 we strengthen this result by observing that the $q$ bracket restricted to $\mathcal{S}$ is surjective on all quasimodular forms. In Section 4.7 we compute the induced product of two moments functions. We study the symmetric algebra $\mathcal{T}$ in Section 4.8, where we also prove our main theorem using the aforementioned computation. The $\mathfrak{s l}_{2}$-action by differential operators, the proof of Theorem 4.1.3, and Rankin-Cohen brackets are the content of Section 4.9 and 4.10. In Section 4.11 further results are given that arise from comparing the two different products on $\mathcal{T}$. Finally, in Section 4.12, we provide many examples of functions in or closely related to $\mathcal{T}$.

\subsection{Preliminaries}

\section{Partitions as a partially ordered set}

Given $n \in \mathbb{Z}_{\geq 0}$, let $\Pi(n)$ be the set of all partitions of the set $[n]:=\{1,2, \ldots, n\}$. For $\alpha \in \Pi(n)$ we write $\ell(\alpha)$ for the cardinality of $\alpha$.

Recall that for $\lambda \in \mathscr{P}$ we have $|\lambda|=\sum_{i=1}^{\infty} \lambda_{i}$, that the length $\ell(\lambda)$ is the largest index $k$ such that $\lambda_{k}>0$ and that $r_{m}(\lambda)$ denotes the number of parts of $\lambda$ equal to $m$, i.e., $r_{m}(\lambda)=\#\left\{i \mid \lambda_{i}=m\right\}$. We call a partition $\lambda$ strict if there are no repeated parts, i.e., $r_{m}(\lambda) \in\{0,1\}$ for all $m$. For two partitions $\kappa, \lambda$ we write $\kappa \cup \lambda$ for the union of $\kappa$ and $\lambda$ as multisets, i.e., $r_{m}(\kappa \cup \lambda)=r_{m}(\kappa)+r_{m}(\lambda)$ for all $m \in \mathbb{N}$.

Both $\mathscr{P}$ and $\Pi(n)$ form a locally finite partially ordered set, i.e., a partially ordered set $P$ for which for all $x, z \in P$ there exists finitely many $y \in P$ such that $x \leq y \leq z$. Namely, on $\mathscr{P}$ we define a partial order by $\kappa \leq \lambda$ if $r_{m}(\kappa) \leq r_{m}(\lambda)$ for all $m \geq 1$. The ordering on $\Pi(n)$ is given by $\alpha \leq \beta$ if for all $A \in \alpha$ there exists a $B \in \beta$ such that $A \subseteq B$. For instance, we have $\alpha \leq \mathbf{1}_{n}$ for all $\alpha \in \Pi(n)$, where $\mathbf{1}_{n}=\{[n]\}$.

Recall that the Möbius function $\mu: P^{2} \rightarrow \mathbb{Z}$ of a locally finite partially ordered set $P$ is defined recursively by (see for example [Rot64]):

$$
\mu(x, z)= \begin{cases}-\sum_{x \leq y<z} \mu(x, y) & x<z \\ 1 & x=z \\ 0 & \text { else. }\end{cases}
$$


For the above partial order on $\mathscr{P}$ the value of $\mu(\kappa, \lambda)$ depends on whether the difference of $\kappa$ and $\lambda$ considered as multisets, denoted by $\lambda-\kappa$, is a strict partition. That is,

$$
\mu(\kappa, \lambda)= \begin{cases}(-1)^{\ell(\lambda)-\ell(\kappa)} & \lambda-\kappa \text { is a strict partition } \\ 0 & \text { else. }\end{cases}
$$

The Möbius function $\mu(\alpha, \beta)$ of two elements $\alpha, \beta \in \Pi(n)$ is given by

$$
\mu(\alpha, \beta)=\prod_{B \in \beta}(-1)^{\ell\left(\alpha_{B}\right)-1}\left(\ell\left(\alpha_{B}\right)-1\right) !,
$$

where $\alpha_{B}$ for $B \subset[n]$ is the partition on $B$ induced by $\alpha$. A Möbius function satisfies the following two properties:

Theorem 4.2.1. Let $f, g$ be functions on a partially ordered set $P$. Then

(i) $\sum_{\alpha \leq \gamma \leq \beta} \mu(\alpha, \gamma)=\delta_{\alpha, \beta}=\sum_{\alpha \leq \gamma \leq \beta} \mu(\gamma, \beta) \quad$ for all $\alpha, \beta \in P$;

(ii) $f(\alpha)=\sum_{\gamma \leq \alpha} g(\gamma) \quad \forall \alpha \in P \quad \Longleftrightarrow \quad g(\beta)=\sum_{\gamma \leq \beta} \mu(\gamma, \beta) f(\gamma) \quad \forall \beta \in P$.

\section{The connected $q$-bracket}

The $q$-bracket is a map $\mathbb{Q}^{\mathscr{P}} \rightarrow \mathbb{Q}[[q]]$ (or $\mathbb{C}^{\mathscr{P}} \rightarrow \mathbb{C}[[q]]$, but we prefer the former). In this section we define the connected $q$-bracket following [CMZ18, Chapter 11], which naturally arises in enumerative geometry when counting connected coverings. In our setting, the connected $q$-bracket turns out to be easier to compute than the usual $q$-bracket.

For $A \subset[n]$ we denote $f_{A}=\prod_{a \in A} f_{a}$.

Definition 4.2.2. Given an integer $n \geq 1$, the connected q-bracket is defined as the multilinear map

$$
\langle\rangle_{q}: \underbrace{\mathbb{Q}^{\mathscr{P}} \otimes \cdots \otimes \mathbb{Q}^{\mathscr{P}}}_{n} \rightarrow \mathbb{Q}[[q]]
$$

extending the $q$-bracket such that for all $f, f_{1}, \ldots, f_{n} \in \mathbb{Q}^{\mathscr{P}}$ any of the following two equivalent conditions hold:

(i) $\left\langle f_{1} \otimes \cdots \otimes f_{n}\right\rangle_{q}=\sum_{\alpha \in \Pi(n)} \mu\left(\alpha, \mathbf{1}_{n}\right) \prod_{A \in \alpha}\left\langle f_{A}\right\rangle_{q}$;

(ii) $\left\langle f_{1} \otimes \cdots \otimes f_{n}\right\rangle_{q}$ is the coefficient of $x_{1} \cdots x_{n}$ in $\log \left\langle\exp \left(\sum_{i=1}^{n} x_{i} f_{i}\right)\right\rangle_{q}$. 
By invoking the Möbius inversion formula (Theorem 4.2.1(ii)) condition (i) in Definition 4.2.2 implies that

$$
\begin{aligned}
\prod_{B \in \beta}\left\langle\otimes_{b \in B} f_{b}\right\rangle_{q} & =\sum_{\alpha \leq \beta} \mu(\alpha, \beta) \prod_{A \in \alpha}\left\langle f_{A}\right\rangle_{q}, \\
\prod_{A \in \alpha}\left\langle f_{A}\right\rangle_{q} & =\sum_{\beta \leq \alpha} \prod_{B \in \beta}\left\langle\otimes_{b \in B} f_{b}\right\rangle_{q} .
\end{aligned}
$$

For example,

$$
\begin{aligned}
\langle f \otimes g\rangle_{q} & =\langle f g\rangle_{q}-\langle f\rangle_{q}\langle g\rangle_{q}, \\
\langle f \otimes g \otimes h\rangle_{q} & =\langle f g h\rangle_{q}-\langle f\rangle_{q}\langle g h\rangle_{q}-\langle g\rangle_{q}\langle f h\rangle_{q}-\langle h\rangle_{q}\langle f g\rangle_{q}+2\langle f\rangle_{q}\langle g\rangle_{q}\langle h\rangle_{q},
\end{aligned}
$$

and

$$
\begin{gathered}
\langle f g\rangle_{q}=\langle f \otimes g\rangle_{q}+\langle f\rangle_{q}\langle g\rangle_{q}, \\
\langle f g h\rangle_{q}=\langle f \otimes g \otimes h\rangle_{q}+\langle f\rangle_{q}\langle g \otimes h\rangle_{q}+\langle g\rangle_{q}\langle f \otimes h\rangle_{q}+ \\
\langle h\rangle_{q}\langle f \otimes g\rangle_{q}+\langle f\rangle_{q}\langle g\rangle_{q}\langle h\rangle_{q} .
\end{gathered}
$$

We often make use of the fact that the connected $q$-bracket of functions $f_{1}, \ldots, f_{n}$ vanishes if one of the $f_{i}$ is constant.

Lemma 4.2.3. For all $f_{1}, \ldots, f_{n} \in \mathbb{Q}^{\mathscr{P}}$ one has

$$
\left\langle 1 \otimes f_{1} \otimes \cdots \otimes f_{n}\right\rangle_{q}=0 .
$$

Proof. Write $f_{n+1}=1$. Observe that $\prod_{A \in \alpha}\left\langle f_{A}\right\rangle_{q}$ takes the same value for all $\alpha \in \Pi(n+1)$ which agree on $[n]$ (but differ in the subset $A$ of $\alpha$ containing $n+1$ ). Then, summing $\mu\left(\alpha, \mathbf{1}_{n}\right)$ over all such $\alpha$ yields

$$
a \cdot(-1)^{a-1}(a-1) !+(-1)^{a} a !=0,
$$

as there are $a$ choices for $\alpha$ for which $\{n+1\}$ is not a subset of $\alpha$, where $a$ is the length of such an $\alpha$, and there is only one choice for $\alpha$ for which $\{n+1\}$ is a subset. By Definition 4.2.2(i) the result follows.

We will use the second condition in Definition 4.2.2 in our proof that $\mathcal{S}$ is a quasimodular algebra. 


\section{The discrete convolution product and Seki-Bernoulli polynomials}

Let $\mathbb{N}$ denote the set of strictly positive integers. Given $f, g: \mathbb{N} \rightarrow \mathbb{Q}$ we denote by $f \cdot g$ or $f g$ the pointwise product of $f$ and $g$. We define the discrete convolution product of $f$ and $g$ by

$$
(f * g)(n)=\sum_{i=1}^{n-1} f(i) g(n-i)
$$

and denote the convolution product of functions $f_{1}, \ldots, f_{n}$ by

$$
\underset{i=1}{*} f_{i}=f_{1} * \cdots * f_{n}
$$

Let the discrete derivative $\partial$ of $f: \mathbb{N} \rightarrow \mathbb{Q}$ be defined by $\partial f(n)=f(n)-f(n-1)$ for $n \geq 2$ and $\partial f(1)=f(1)$ and denote by id the identity function $\mathbb{N} \rightarrow \mathbb{N} \subset \mathbb{Q}$. Observe that

$$
\begin{aligned}
\partial(f * g) & =(\partial f) * g=f *(\partial g), \\
\partial(f g) & =\partial(f) g+f \partial(g)-\partial(f) \partial(g), \\
\text { id } \cdot(f * g) & =(\text { id } \cdot f) * g+f *(\mathrm{id} \cdot g), \\
\partial^{2}(f * \mathrm{id}) & =f-\partial f .
\end{aligned}
$$

The Seki-Bernoulli polynomials ${ }^{1} \mathcal{F}_{l}$ for $l \geq 1$ are defined as the unique polynomials with vanishing constant term satisfying $\partial \mathcal{F}_{l}(n)=n^{l-1}$ for all $n \in \mathbb{N}$, or equivalently by $\mathcal{F}_{l}(n)=\sum_{i=1}^{n} i^{l-1}$. The first four are given by $\mathcal{F}_{1}(x)=x$ and

$$
\mathcal{F}_{2}(x)=\frac{x(x+1)}{2}, \quad \mathcal{F}_{3}(x)=\frac{x(x+1)(2 x+1)}{6}, \quad \mathcal{F}_{4}(x)=\frac{x^{2}(x+1)^{2}}{4} .
$$

Note that these polynomials are related to the Bernoulli polynomials $B_{n}(x)$, the unique family of polynomials satisfying $\int_{x}^{x+1} B_{n}(u) \mathrm{d} u=x^{n}$, by the formula

$$
l \mathcal{F}_{l}(x)=B_{l}(x+1)-B_{l} .
$$

Hence, the Seki-Bernoulli polynomials admit the symmetry

$$
\mathcal{F}_{l}(x)=(-1)^{l} \mathcal{F}_{l}(-x-1) \quad(l \geq 2),
$$

which can also be deduced directly from the definition. The generating series $\mathcal{F}(n)$ of the Seki-Bernoulli polynomials equals

$$
\mathcal{F}(n):=\sum_{l=1}^{\infty} \mathcal{F}_{l}(n) \frac{z^{l-1}}{(l-1) !}=e^{z} \frac{1-e^{n z}}{1-e^{z}} .
$$

\footnotetext{
${ }^{1}$ These polynomials are usually called Faulhaber polynomials. As often in mathematics, this name does not reflect the correct history; see [AIK14, p. 3].
} 


\subsection{Three proofs of the quasimodularity of the moment functions}

The $q$-bracket of the moment function $S_{k}$ defined in (4.1) equals the Eisenstein series $G_{k}$. To motivate the results in the rest of this chapter, we provide three different proofs - and three generalisations — of this statement using three different approaches. In the first approach we motivate the definition of the $T_{k, l}$ (see (4.2)), the second approach gives an interpretation for these functions, and the last approach gives an example of our main principle of establishing all identities before taking the $q$-bracket.

\section{First approach}

The key observation in this first proof is that $S_{k}$ can be rewritten as

$$
S_{k}(\lambda)=-\frac{B_{k}}{2 k}+\sum_{m=1}^{\infty} m^{k-1} r_{m}(\lambda)
$$

More generally, for $k>0$ and $f: \mathbb{N} \rightarrow \mathbb{Q}$ we set $f(0)=0$ and we let

$$
S_{k, f}(\lambda)=-\frac{B_{k+1}}{2(k+1)} \delta_{f, \text { id }}+\sum_{m=1}^{\infty} m^{k} f\left(r_{m}(\lambda)\right) .
$$

In case when $f$ is the identity, $S_{k, f}=S_{k+1}$. Our first method of proof gives the following more general statement:

Proposition 4.3.1. Let $f$ be a polynomial of degree $l$ without constant term and $k a$ positive integer satisfying $k \equiv l \bmod 2$. Then,

(i) if $f$ equals a Seki-Bernoulli polynomial $\mathcal{F}_{l}$, then $\left\langle S_{k, f}\right\rangle_{q}$ equals

$$
-\frac{B_{k+1}}{2(k+1)} \delta_{l, 1}+\sum_{m, r \geq 1} m^{k} r^{l-1} q^{m r}= \begin{cases}D^{l-1} G_{k-l+2} & k-l \geq 0 \\ D^{k} G_{l-k} & k-l \leq 2\end{cases}
$$

(ii) if $\left\langle S_{k, f}\right\rangle_{q}$ is a quasimodular form, then $f$ is a multiple of the Seki-Bernoulli polynomial $\mathcal{F}_{l}$.

Proof. Let $|x| \leq 1$ and $m \geq 1$. We compute

$$
\left\langle x^{r_{m}}\right\rangle_{q}=\frac{\sum_{\lambda \in \mathscr{P}} x^{r_{m}(\lambda)} q^{|\lambda|}}{\sum_{\lambda \in \mathscr{P}} q^{|\lambda|}} .
$$


Observe that the multiplicities $r_{1}(\lambda), r_{2}(\lambda), \ldots$ uniquely determine the partition $\lambda$. Hence, for $|q|<1$ we have that

$$
\begin{aligned}
\sum_{\lambda \in \mathscr{P}} x^{r_{m}(\lambda)} q^{|\lambda|} & =\sum_{r_{1}, r_{2}, \ldots \geq 0} x^{r_{m}} q^{r_{1}+2 r_{2}+\ldots+m r_{m}+\ldots} \\
& =\left(\sum_{r_{m}=0}^{\infty} x^{r_{m}} q^{m r_{m}}\right) \prod_{i \neq m}\left(\sum_{r_{i}=0}^{\infty} q^{i r_{i}}\right) \\
& =\frac{1}{1-x q^{m}} \prod_{i \neq m} \frac{1}{1-q^{i}} .
\end{aligned}
$$

Substituting this result in the numerator of (4.12), we obtain

$$
\left\langle x^{r_{m}}\right\rangle_{q}=\frac{1-q^{m}}{1-x q^{m}} .
$$

Hence,

$$
\left\langle\frac{x}{1-x}\left(1-x^{r_{m}}\right)\right\rangle_{q}=\frac{x q^{m}}{1-x q^{m}} .
$$

Observe that applying $x \frac{\partial}{\partial x}$ to the right-hand side of (4.13) has the same effect as applying $\frac{1}{m} D$. After setting $x=e^{z}$, we find that $\frac{x}{1-x}\left(1-x^{r_{m}}\right)$ equals $\mathcal{F}\left(r_{m}\right)$ (see (4.10)). Hence, by taking $l-1$ derivatives $x \frac{\partial}{\partial x}=\frac{\partial}{\partial z}$ and setting $z=0$, it follows that

$$
\begin{aligned}
\left\langle S_{k, \mathcal{F}_{l}}\right\rangle_{q}+\frac{B_{k+1}}{2(k+1)} \delta_{l, 1} & =\sum_{m \geq 0} m^{k}\left\langle\mathcal{F}_{l}\left(r_{m}\right)\right\rangle_{q} \\
& =\left.\sum_{m \geq 0} m^{k}\left(x \frac{\partial}{\partial x}\right)^{l-1} \frac{x q^{m}}{1-x q^{m}}\right|_{x=1} \\
& =\sum_{m \geq 0} m^{k}\left(\frac{1}{m} D\right)^{l-1} \frac{q^{m}}{1-q^{m}} \\
& =\sum_{m, r \geq 1} m^{k} r^{l-1} q^{m r}
\end{aligned}
$$

Part (ii) of the statement follows by writing $f$ as a linear combination of SekiBernoulli polynomials. 


\section{Second approach}

The double moment functions $T_{k, l}$ (see (4.2)) are by definition equal to $S_{k, \mathcal{F}_{l}}$ if $k>0$. Given a partition $\lambda$, let $c_{i}(\lambda)=\#\left\{j \leq i \mid \lambda_{i}=\lambda_{j}\right\}$. Then, one has

$$
T_{k, l}(\lambda)=-\frac{B_{k+l}}{2(k+l)}\left(\delta_{l, 1}+\delta_{k, 0}\right)+\sum_{i=1}^{\infty} \lambda_{i}^{k} c_{i}(\lambda)^{l-1} .
$$

In this section we give a direct proof for the quasimodularity of the $q$-brackets of $T_{k, l}$ :

Proposition 4.3.2. For all $k \geq 0, l \geq 1$ and $k+l$ even one has

$$
\left\langle T_{k, l}\right\rangle_{q}= \begin{cases}D^{l-1} G_{k-l+2} & \text { if } k-l \geq 0 \\ D^{k} G_{l-k} & \text { if } k-l \leq 2 .\end{cases}
$$

Proof. Denote by $T_{k, l}^{0}(\lambda)=\sum_{i=1}^{\infty} \lambda_{i}^{k} c_{i}(\lambda)^{l-1}$. The generating series of $T_{k, l}^{0}$ is given by

$$
W(X, Y)(\lambda)=\sum_{i=1}^{\infty} X^{\lambda_{i}} Y^{c_{i}(\lambda)},
$$

that is, $T_{k, l}^{0}(\lambda)$ is the coefficient of $\frac{x^{k} y^{l-1}}{k !(l-1) !}$ in $W\left(e^{x}, e^{y}\right)(\lambda)$. Consider

$$
\sum_{\lambda \in \mathscr{P}} W(X, Y)(\lambda) q^{|\lambda|}=\sum_{\lambda \in \mathscr{P}} \sum_{i=1}^{\infty} X^{\lambda_{i}} Y^{c_{i}(\lambda)} q^{|\lambda|} .
$$

Given $a, b, n \in \mathbb{Z}_{\geq 0}$, denote by $C_{a, b}(n)$ the coefficient in front of $X^{a} Y^{b} q^{n}$ in (4.14), that is

$$
\sum_{\lambda \in \mathscr{P}} \sum_{i=1}^{\infty} X^{\lambda_{i}} Y^{c_{i}(\lambda)} q^{|\lambda|}=: \sum_{a, b, n \geq 0} C_{a, b}(n) X^{a} Y^{b} q^{n} .
$$

Recall $p(n)$ denotes the number of partitions of $n$. The coefficient $C_{a, b}(n)$ equals the number of partitions of $n$ with at least $b$ parts of size $a$, i.e., $C_{a, b}(n)=p(n-a b)$. Hence, writing $m=n-a b$ we obtain

$$
\sum_{\lambda \in \mathscr{P}} \sum_{i=1}^{\infty} X^{\lambda_{i}} Y^{c_{i}(\lambda)} q^{|\lambda|}=\left(\sum_{m=0}^{\infty} p(m) q^{m}\right)\left(\sum_{a, b \geq 0} X^{a} Y^{b} q^{a b}\right) .
$$

In other words,

$$
\langle W(X, Y)\rangle_{q}=\sum_{a, b \geq 0} X^{a} Y^{b} q^{a b}
$$


so that expanding this equation for $X=e^{x}$ and $Y=e^{y}$ yields

$$
\left\langle T_{k, l}^{0}\right\rangle_{q}=\sum_{a, b \geq 0} a^{k} b^{l-1} q^{a b} .
$$

As $T_{k, l}(\lambda)=-\frac{B_{k+l}}{(k+l)}\left(\delta_{l, 1}+\delta_{k, 0}\right)+T_{k, l}^{0}(\lambda)$ we obtain the desired result.

\section{Third approach}

In this last proof we start with the observation that one can rewrite the $q$-bracket as

$$
\langle f\rangle_{q}=\left.\frac{\sum_{\lambda \in \mathscr{P}} f(\lambda) u_{\lambda_{1}} u_{\lambda_{2}} \cdots}{\sum_{\lambda \in \mathscr{P}} u_{\lambda_{1}} u_{\lambda_{2}} \cdots}\right|_{u_{i}=q^{i}} .
$$

In contrast to the previous two proofs, it is only in the last step of this proof that we take the $q$-bracket: first we rewrite (4.15) considering $u_{1}, u_{2}, \ldots$ to be formal variables, and in the last step we let $u_{i}=q^{i}$. We start with the denominator, where we encounter the Möbius function on partitions going back to Alladi; see [Sch17, p. 5].

Proposition 4.3.3. There exists a function $\mu: \mathscr{P} \rightarrow\{-1,0,1\}$ defined by any one of the following three equivalent definitions:

(i) $\mu(\lambda)$ is given by the Möbius function $\mu(\emptyset, \lambda)$ on the partial order on the set of partitions in (4.3);

(ii) $\mu(\lambda)= \begin{cases}(-1)^{\ell(\lambda)} & \lambda \text { is a strict partition } \\ 0 & \text { else; }\end{cases}$

(iii) $\frac{1}{\sum_{\lambda \in \mathscr{P}} u_{\lambda_{1}} u_{\lambda_{2}} \cdots}=\sum_{\lambda \in \mathscr{P}} \mu(\lambda) u_{\lambda_{1}} u_{\lambda_{2}} \cdots$.

Proof. The first two definitions clearly coincide using (4.3). For the latter, it suffices to show that

$$
\sum_{\alpha \cup \beta=\lambda} \mu(\alpha)=\delta_{\lambda, \emptyset}
$$

Let $f(\lambda)=1$ and $g(\lambda)=\delta_{\lambda, \emptyset}$ for $\lambda \in \mathscr{P}$. Then, $f(\alpha)=\sum_{\gamma \leq \alpha} g(\gamma)$ for all $\alpha \in \mathscr{P}$, so that by Möbius inversion and by using $\mu(\gamma, \beta)=\mu(\emptyset, \beta-\gamma)$ the last definition is equivalent.

The fact that $\left\langle S_{k}\right\rangle_{q}=G_{k}$ follows directly from the following proposition: 
Proposition 4.3.4. For all $m \geq 1$ and $f: \mathbb{N} \rightarrow \mathbb{Q}$ extended by $f(0)=0$ one has

$$
\frac{\sum_{\lambda \in \mathscr{P}} f\left(r_{m}(\lambda)\right) u_{\lambda_{1}} u_{\lambda_{2}} \cdots}{\sum_{\lambda \in \mathscr{P}} u_{\lambda_{1}} u_{\lambda_{2}} \cdots}=\sum_{r=1}^{\infty} \partial f(r) u_{m}^{r} .
$$

Proof. Fix $m \geq 1$. Write $u_{\lambda}=u_{\lambda_{1}} u_{\lambda_{2}} \ldots$. By the previous proposition, we have

$$
\frac{\sum_{\lambda \in \mathscr{P}} f\left(r_{m}(\lambda)\right) u_{\lambda}}{\sum_{\lambda \in \mathscr{P}} u_{\lambda}}=\left(\sum_{\lambda \in \mathscr{P}} f\left(r_{m}(\lambda)\right) u_{\lambda}\right)\left(\sum_{\lambda \in \mathscr{P}} \mu(\lambda) u_{\lambda}\right) .
$$

Denote by $C(\lambda)$ the coefficient of $u_{\lambda}$ after expanding the right-hand side of above equation. Observe that

$$
C(\lambda)=\sum_{\alpha \cup \beta=\lambda}(-1)^{\ell(\beta)} f\left(r_{m}(\alpha)\right),
$$

where $\alpha \cup \beta$ denotes the union of $\alpha$ and $\beta$ considered as multisets and it is understood that $\beta$ is a strict partition. Suppose $\lambda$ admits a part equal to $m^{\prime} \neq m$. Then, define an involution $\omega$ on all pairs $(\alpha, \beta)$ satisfying that $\alpha \cup \beta=\lambda$ and $\beta$ is strict by

$$
\omega(\alpha, \beta)= \begin{cases}\left(\alpha \backslash\left\{m^{\prime}\right\}, \beta \cup\left\{m^{\prime}\right\}\right) & \text { if } r_{m^{\prime}}(\beta)=0 \\ \left(\alpha \cup\left\{m^{\prime}\right\}, \beta \backslash\left\{m^{\prime}\right\}\right) & \text { if } r_{m^{\prime}}(\beta)=1 .\end{cases}
$$

As $\omega$ changes the sign of $(-1)^{\ell(\beta)} f\left(r_{m}(\alpha)\right)$, it follows that $C(\lambda)=0$.

Observe that $C(\emptyset)=0$ and that in case $\lambda=(m, m, \ldots)$ consists of a strictly positive number of parts all equal to $m$ one has

$$
C(\lambda)=f\left(r_{m}(\lambda)\right)-f\left(r_{m}(\lambda)-1\right)=\partial f\left(r_{m}(\lambda)\right) .
$$

Therefore, the desired result follows.

\subsection{The induced and connected product}

Motivated by the last of the three approaches in the previous section, we define the $\boldsymbol{u}$ bracket of a function $f \in \mathbb{Q}^{\mathscr{P}}$ by

$$
\langle f\rangle_{\boldsymbol{u}}=\frac{\sum_{\lambda \in \mathscr{P}} f(\lambda) u_{\lambda}}{\sum_{\lambda \in \mathscr{P}} u_{\lambda}} \quad\left(u_{\lambda}=u_{\lambda_{1}} u_{\lambda_{2}} \cdots\right) .
$$

Then, for all $f \in \mathbb{Q}^{\mathscr{P}}$ one has $\langle f\rangle_{q}=\langle f\rangle_{\left(q, q^{2}, q^{3}, \ldots\right)}$. Observe that the $\boldsymbol{u}$-bracket defines an isomorphism of vector spaces

$$
\mathbb{Q}^{\mathscr{P}} \stackrel{\sim}{\rightarrow} \mathbb{Q}\left[\left[u_{1}, u_{2}, u_{3}, \ldots\right]\right], \quad f \mapsto\langle f\rangle_{\boldsymbol{u}} .
$$

We now use the algebra structure of $\mathbb{Q}\left[\left[u_{1}, u_{2}, u_{3}, \ldots\right]\right]$ to define a product on $\mathbb{Q}^{\mathscr{P}}$. 
Definition 4.4.1. Given $f, g \in \mathbb{Q}^{\mathscr{P}}$ we define their induced product $f \odot g$ by

$$
\langle f \odot g\rangle_{\boldsymbol{u}}=\langle f\rangle_{\boldsymbol{u}}\langle g\rangle_{\boldsymbol{u}},
$$

where the product of $\langle f\rangle_{\boldsymbol{u}}$ and $\langle g\rangle_{\boldsymbol{u}}$ is the usual product of power series.

Remark 4.4.2. Observe that $\mathbb{Q}^{\mathscr{P}}$ is a commutative algebra with the constant function 1 as the identity for both the pointwise and the induced product. This observation should be compared with the $q$-bracket arithmetic in [Sch17].

The following proposition gives an alternative definition for the induced product.

Proposition 4.4.3. For all $\lambda \in \mathscr{P}$ one has

$$
(f \odot g)(\lambda)=\sum_{\alpha \cup \beta \cup \gamma=\lambda} f(\alpha) g(\beta) \mu(\gamma) .
$$

Proof. By definition

$$
\sum_{\lambda \in \mathscr{P}}(f \odot g)(\lambda) u_{\lambda}=\frac{\left(\sum_{\lambda \in \mathscr{P}} f(\lambda) u_{\lambda}\right)\left(\sum_{\lambda \in \mathscr{P}} g(\lambda) u_{\lambda}\right)}{\sum_{\lambda \in \mathscr{P}} u_{\lambda}} .
$$

By Proposition 4.3.3 this equals

$$
\left(\sum_{\lambda \in \mathscr{P}} f(\lambda) u_{\lambda}\right)\left(\sum_{\lambda \in \mathscr{P}} g(\lambda) u_{\lambda}\right)\left(\sum_{\lambda \in \mathscr{P}} \mu(\lambda) u_{\lambda}\right) .
$$

The result follows by expanding the products.

Analogous to the connected $q$-bracket, we define the connected product. For a set $S$ and functions $f_{s} \in \mathbb{Q}^{\mathscr{P}}$ for all $s \in S$, we denote $f_{S}=\prod_{s \in S} f_{s}$.

Definition 4.4.4. For $f_{1}, \ldots, f_{n} \in \mathbb{Q}^{\mathscr{P}}$, define the connected product $f_{1}|\ldots| f_{n}$ to be the following function $\mathscr{P} \rightarrow \mathbb{Q}$ :

$$
f_{1}|\ldots| f_{n}:=\sum_{\alpha \in \Pi(n)} \mu(\alpha, \mathbf{1}) \bigodot_{A \in \alpha} f_{A} .
$$

For example, for $f, g, h \in \mathbb{Q}^{\mathscr{P}}$ one has

$$
\begin{aligned}
f \mid g & =f g-f \odot g, \\
f|g| h & =f g h-f \odot g h-g \odot f h-h \odot f g+2 f \odot g \odot h .
\end{aligned}
$$

The induced and connected product allow us to establish many identities before taking the $q$-bracket, as follows from the following result. 
Proposition 4.4.5. For all $f_{1}, \ldots, f_{n} \in \mathbb{Q}^{\mathscr{P}}$ one has

- $\left\langle f_{1} \odot f_{2} \odot \cdots \odot f_{n}\right\rangle_{q}=\left\langle f_{1}\right\rangle_{q}\left\langle f_{2}\right\rangle_{q} \cdots\left\langle f_{n}\right\rangle_{q} ;$

- $\left\langle f_{1}|\ldots| f_{n}\right\rangle_{q}=\left\langle f_{1} \otimes \cdots \otimes f_{n}\right\rangle_{q}$.

Proof. Both statements follow directly from the definitions. For the first, note that for all $f, g \in \mathbb{Q}^{\mathscr{P}}$ one has

$$
\langle f \odot g\rangle_{q}=\left.\langle f\rangle_{\boldsymbol{u}}\langle g\rangle_{\boldsymbol{u}}\right|_{u_{i}=q^{i}}=\left.\left.\langle f\rangle_{\boldsymbol{u}}\right|_{u_{i}=q^{i}}\langle g\rangle_{\boldsymbol{u}}\right|_{u_{i}=q^{i}}=\langle f\rangle_{q}\langle g\rangle_{q},
$$

so that the statement follows inductively. The second follows from the first, as

$$
\left\langle f_{1}|\ldots| f_{n}\right\rangle_{q}=\sum_{\alpha \in \Pi(n)} \mu(\alpha, \mathbf{1}) \prod_{A \in \alpha}\left\langle f_{A}\right\rangle_{q}=\left\langle f_{1} \otimes \cdots \otimes f_{n}\right\rangle_{q} .
$$

Remark 4.4.6. Let $\mathcal{R}$ be the space of functions having a quasimodular form as $q$ bracket, i.e. $\mathcal{R}=\langle\cdot\rangle_{q}^{-1}(\widetilde{M})$. Then, $\mathcal{R}$ is a graded algebra with multiplication given by the induced product. Namely, if $f \in \mathcal{R}$ and $\langle f\rangle_{q} \in \widetilde{M}_{k}$, we define the weight of $f$ to be equal to $k$. Note that if $f, g \in \mathcal{R}$ and $\langle f\rangle_{q}$ and $\langle g\rangle_{q}$ are quasimodular forms of weight $k$ and $l$, respectively, then $\langle f \odot g\rangle_{q}=\langle f\rangle_{q}\langle g\rangle_{q}$ is a quasimodular form of weight $k+l$.

When establishing identities on the level of functions on partitions (before taking the $q$-bracket), it turns out to be very useful to express the connected product of pointwise products of elements of $\mathbb{Q}^{\mathscr{P}}$ in terms of connected and induced products. This can be done recursively using the following result.

Proposition 4.4.7. For all $f_{1}, \ldots f_{n} \in \mathbb{Q}^{\mathscr{P}}$ one has

$$
\begin{aligned}
& f_{1} f_{2}\left|f_{3}\right| f_{4}|\ldots| f_{n}= f_{1}\left|f_{2}\right| \ldots \mid f_{n}+ \\
& \sum_{A \sqcup B=\{3, \ldots, n\}}\left(f_{1}\left|f_{A_{1}}\right| f_{A_{2}} \mid \ldots\right) \odot\left(f_{2}\left|f_{B_{1}}\right| f_{B_{2}} \mid \ldots\right),
\end{aligned}
$$

where $A_{1}, A_{2}, \ldots$ enumerate the elements of $A$ (and similarly for $B$ ).

Proof. Observe that both sides of the equation in the statement are a linear combination of terms of the form $\bigodot_{C \in \gamma} f_{C}$ over $\gamma \in \Pi(n)$. We determine the coefficient of such a term on both sides of the equation.

First of all, assume $\gamma$ is such that $\{1,2\} \subset C$ for some $C \in \gamma$. Then, on the right-hand side such a term only occurs in $f_{1}|\ldots| f_{n}$ with coefficient $\mu(\gamma, \mathbf{1})$. Moreover, let $\tilde{\gamma} \in \Pi(n-1)$ be given by $\gamma \cap\{2, \ldots, n\}$ subject to replacing $i$ by $i-1$ for all $i=2, \ldots, n$. Note that the coefficient on the left-hand side equals $\mu(\tilde{\gamma}, \mathbf{1})$. As $\ell(\tilde{\gamma})=\ell(\gamma)$, the coefficients on both sides agree. 
Next, assume $C_{1}, C_{2} \in \gamma$ with $1 \in C_{1}$ and $2 \in C_{2}$. Then, the coefficient of $\bigodot_{C \in \gamma} f_{C}$ on right-hand side of (4.17) equals

$$
\mu(\gamma, \mathbf{1})+\sum \mu\left(\left.\gamma\right|_{A}, \mathbf{1}\right) \mu\left(\left.\gamma\right|_{B}, \mathbf{1}\right),
$$

where the sum is over all $I \subset\{2,3, \ldots, \ell(\gamma)\}$ and $A$ and $B$ are given by $A=$ $C_{1} \cup \bigcup_{i \in I} C_{i}$ and $B=C_{2} \cup \bigcup_{i \in I^{c}} C_{i}$. Letting $i$ be the number of elements of $I$, we find that (4.18) equals

$$
\begin{aligned}
& \mu(\gamma, \mathbf{1})+\sum_{i=0}^{\ell(\gamma)-2}\left(\begin{array}{c}
\ell(\gamma)-2 \\
i
\end{array}\right) \cdot(-1)^{i} i ! \cdot(-1)^{\ell(\gamma)-i-2}(\ell(\gamma)-i-2) ! \\
= & \mu(\gamma, \mathbf{1})+\sum_{i=0}^{\ell(\gamma)-2}(\ell(\gamma)-2) !(-1)^{\ell(\gamma)-2} \\
= & \mu(\gamma, \mathbf{1})-\mu(\gamma, \mathbf{1})=0 .
\end{aligned}
$$

Correspondingly, the coefficient of $\bigodot_{C \in \gamma} f_{C}$ on the left-hand side of (4.17) vanishes if there are $C_{1}, C_{2} \in \gamma$ with $1 \in C_{1}$ and $2 \in C_{2}$.

\subsection{Quasimodularity of pointwise products of moment functions}

Not only do the moment functions $S_{k}$ admit quasimodular $q$-brackets, but also the homogeneous polynomials in the moment functions admit quasimodular $q$-brackets; here, each moment function $S_{k}$ has weight $k$ in accordance with the fact that $\left\langle S_{k}\right\rangle_{q}$ has weight $k$. Given a tuple $\boldsymbol{k}=\left(k_{1}, \ldots, k_{n}\right)$ of even integers, we write $S_{\boldsymbol{k}}=$ $S_{k_{1}} \cdots S_{k_{n}}$. Note that, as a vector space, $\mathcal{S}$ is spanned by these functions $S_{\boldsymbol{k}}$. We provide two approaches to proving the quasimodularity of the $q$-brackets of the $S_{k}$. First, we give a direct proof of the statement in Theorem 4.5.1, after which, in accordance with our main principle of establishing all identities before taking the $q$ bracket, we prove a more general result which will be used frequently in the next section.

Theorem 4.5.1. The algebra $\mathcal{S}$ is a quasimodular algebra. More precisely, for $\boldsymbol{k} \in$ $(2 \mathbb{N})^{n}$ one has

$$
\left\langle S_{\boldsymbol{k}}\right\rangle_{q}=\sum_{\alpha \in \Pi(n)} \prod_{A \in \alpha} D^{\ell(A)-1} G_{\left|\boldsymbol{k}_{A}\right|-2 \ell(A)+2} .
$$

Proof. Observe that it suffices to show that

$$
\left\langle\bigotimes_{k \in \boldsymbol{k}} S_{k}\right\rangle_{q}=D^{n-1} G_{|\boldsymbol{k}|-2 n+2}
$$


as (4.19) follows from (4.20) by Möbius inversion. Recall that $\left\langle f_{1} \otimes \cdots \otimes f_{n}\right\rangle_{q}$ is the coefficient of $x_{1} \cdots x_{n}$ in $\log \left\langle\exp \left(\sum_{i=1}^{n} x_{i} f_{i}\right)\right\rangle_{q}$ (see Definition 4.2.2(ii)). Consider $S_{k}^{0}(\lambda)=\sum_{i=1}^{\infty} \lambda_{i}^{k-1}$ for all positive even $k$. Euler's formula for the generating series of partitions (1.1) follows from writing $|\lambda|=\sum_{m \geq 1} m r_{m}(\lambda)$ and summing over all possible values of $r_{1}(\lambda), r_{2}(\lambda), \ldots$ By the same idea, we find

$$
\sum_{\lambda \in \mathscr{P}} \exp \left(\sum_{k} S_{k}^{0}(\lambda) x_{k}\right) q^{|\lambda|}=\prod_{m=1}^{\infty}\left(1-\exp \left(\sum_{k} m^{k-1} x_{k}\right) q^{m}\right)^{-1} .
$$

The logarithm of this expression equals

$$
\sum_{m, r=1}^{\infty} \exp \left(r \sum_{k} m^{k-1} x_{k}\right) \frac{q^{m r}}{r} .
$$

Now, assume all parts of $\boldsymbol{k}$ are distinct. In the expansion of (4.22) the coefficient of $x_{k_{1}} \cdots x_{k_{n}}$ equals

$$
\sum_{m, r=1}^{\infty} m^{|\boldsymbol{k}|-n} r^{n-1} q^{m r}=D^{n-1} G_{|\boldsymbol{k}|-2 n+2} .
$$

Hence,

$$
\left\langle\bigotimes_{k \in \boldsymbol{k}} S_{k}^{0}\right\rangle_{q}=D^{n-1} G_{|\boldsymbol{k}|-2 n+2}
$$

By introducing distinct variables in Eq. (4.21) for each repeated part of $\boldsymbol{k}$ we obtain the same result if not all parts of $\boldsymbol{k}$ are distinct.

Note that if $n \geq 2$, by Lemma 4.2.3 both sides of the equation do not change if one replaces $S_{k}^{0}$ by $S_{k}$. In case $n=1$ we have established (4.20) in Proposition 4.3.1 or in Proposition 4.3.2. Hence, (4.20) holds and (4.19) is then implied by Möbius inversion.

Denote

$$
p_{k}(z)= \begin{cases}\frac{z^{k-2}}{(k-2) !} & k \geq 0 \\ \frac{z^{-2}}{2} & k=0\end{cases}
$$

and set $S_{0}(\lambda) \equiv 1$. In terms of the generating series corresponding to the Eisenstein series, called the propagator, and given by

$$
P(\tau, z):=\frac{1}{z^{2}}+2 \sum_{k=2}^{\infty} G_{k} \frac{z^{k-2}}{(k-2) !}
$$

one has the following expression for the generating series of the $q$-bracket of the generators of $\mathcal{S}$ : 


\section{Corollary 4.5.2.}

$$
\sum_{k_{1}, \ldots, k_{n} \geq 0}\left\langle S_{k_{1}} \cdots S_{k_{n}}\right\rangle_{q} p_{k_{1}}\left(z_{1}\right) \cdots p_{k_{n}}\left(z_{n}\right)=\sum_{\alpha \in \Pi(n)} \prod_{A \in \alpha} D^{|A|-1} \frac{P^{\text {even }}\left(\tau ; z_{A}\right)}{2},
$$

where $z_{A}=\sum_{a \in A} z_{a}$ and

$$
P^{\text {even }}\left(\tau ; z_{1}, \ldots, z_{n}\right)=\frac{1}{2^{n}} \sum_{s \in\{-1,1\}^{n}} P\left(\tau, s_{1} z_{1}+\ldots+s_{n} z_{n}\right)
$$

is the totally even part of the propagator in (4.23).

Remark 4.5.3. The propagator is closely related to the Weiserstrass $\wp$-function by

$$
P(z)=\frac{1}{2 \pi i} \wp\left(\frac{z}{2 \pi i}, \tau\right)+2 G_{2} .
$$

In fact, $P$ equals the meromorphic Jacobi form $E_{2}$, introduced in Chapter 5 , up to a constant.

\subsection{Intermezzo: surjectivity of the $q$-bracket}

We deduce from Theorem 4.5.1 the surjectivity of the $q$-bracket: every quasimodular form is the $q$-bracket of some $f \in \mathcal{S}$.

Theorem 4.6.1. The q-bracket $\langle\cdot\rangle_{q}: \mathcal{S} \rightarrow \widetilde{M}$ is surjective.

Note that this is not obvious since the $q$-bracket is not an algebra homomorphism. Denote by $\vartheta_{k}: M_{k} \rightarrow M_{k+2}$ the Serre derivative, given by $\vartheta_{k}=D+2 k G_{2}$. Extend this notation by letting $\vartheta_{x}: \widetilde{M} \rightarrow \widetilde{M}$ for $x \in \mathbb{Q}$ be given by $\vartheta_{x}=D+2 x G_{2}$.

Proposition 4.6.2. Let $x \in \mathbb{Q} \backslash 2 \mathbb{Z}_{\geq 0}$. Then,

$$
\widetilde{M}_{k}^{(\leq p)}=\bigoplus_{r=0}^{p} \vartheta_{x}^{r} M_{k-2 r} .
$$

Proof. Let $f \in M_{k}$ with $f \neq 0$. Observe that $\vartheta_{x} f$ is modular precisely if $k=$ $x$. By our assumption on $x$, this is not the case. Hence, $\vartheta_{x}$ increases the depth strictly by one. The result follows by induction on $p$ by the same argument as in [Zag08, Proposition 20]. Namely, if $\varphi \in \widetilde{M}_{k}^{\leq p}$, then the last coefficient $\varphi_{p}$ in the quasimodular transformation (1.6) is a modular form of weight $k-2 p$. Hence, $\varphi$ is a linear combination of $\vartheta_{x}^{p} \varphi_{p}$ and a quasimodular form of depth strictly smaller than $p$. 
Proof of Theorem 4.6.1. First observe that $\left(D+G_{2}\right)\langle f\rangle_{q}=\left\langle S_{2} f\right\rangle_{q}$. As $D+G_{2}$ is not a Serre derivative, by Proposition 4.6.2 it follows that it suffices to show that the $q$-bracket is surjective on modular forms. Every modular form can be written as a polynomial of degree at most 2 in Eisenstein series, see [Zag77, Section 5]. Hence, we show that the $q$-bracket is surjective on polynomials of degree at most 2 in all Eisenstein series, possibly involving the quasimodular Eisenstein series $G_{2}$.

Eisenstein series are in the image of the $q$-bracket by Theorem 4.5.1. Note that $D G_{k}$ can be written a polynomial of degree 2 in Eisenstein series, explicitly:

$$
D G_{k}=\frac{k+3}{2(k+1)} G_{k+2}-\sum_{\substack{0<j<k \\
j \equiv 1(2)}}\left(\begin{array}{l}
k \\
j
\end{array}\right) G_{j+1} G_{k+1-j} .
$$

Also, we have an explicit formula for the $q$-bracket of $S_{k} S_{l}$ :

$$
\left\langle S_{k} S_{l}\right\rangle_{q}=G_{k} G_{l}+D G_{k+l-2},
$$

so that this $q$-bracket is expressible as a polynomial of degree at most 2 in the Eisenstein series.

Now fix an integer $m \geq 4$. We consider the equations (4.24) for all $k+l=m$. It suffices to show that we can invert these equations, i.e., write $G_{k} G_{l}$ as a linear combination of $q$-brackets of products of at most two $S_{i}$. A direct computation shows that the determinant of the matrix corresponding to the equations above equals

$$
1-\sum_{\substack{0<j<m \\
j \equiv 1(2)}}\left(\begin{array}{c}
m \\
j
\end{array}\right)=1-2^{m-3}<0 .
$$

Hence, the $q$-bracket is surjective.

Remark 4.6.3. Only the last step of above proof uses the explicit formula (4.24) for the derivative of Eisenstein series. The author expects one could conclude the proof by an abstract argument, but he is not aware of such an argument.

\subsection{The connected product of moment functions}

In the second approach we compute the connected product $S_{k_{1}}|\ldots| S_{k_{n}}$, which by Proposition 4.4.5 yields the left-hand side of (4.19) after taking the $q$-bracket. The result is formulated in Theorem 4.7.4 below and depends on two technical lemma's which we state first. 
In order to do so, we start by introducing the following notation. For a partition $\lambda$ and a subset $A$ of $\mathbb{N}$, we write $\left.\lambda\right|_{A}$ for the partition where a part of size $m$ occurs $r_{m}(\lambda)$ times if $m \in A$ and does not occur if $m \notin A$. For example,

$$
\left.(5,4,3,3,1,1,1)\right|_{\{4,1\}}=(4,1,1,1) .
$$

Definition 4.7.1. We say $f: \mathscr{P} \rightarrow \mathbb{Q}$ is supported on $A$ if $f(\lambda)=f\left(\left.\lambda\right|_{A}\right)$ for all partitions $\lambda$.

The first lemma expresses the induced product of two functions $F$ and $G$ supported on disjoint sets as the pointwise product of these functions, and of two functions $F$ and $G$ supported on the same singleton set as a convolution product of functions.

Lemma 4.7.2. Suppose $X$ and $Y$ are subsets of $\mathbb{N}$ and $F, F^{\prime}, G, G^{\prime}: \mathscr{P} \rightarrow \mathbb{Q}$ are supported on $X, X, Y$ and $Y$, respectively. Then

(i) $F \odot F^{\prime}$ is supported on $X$;

(ii) If $X$ and $Y$ are disjoint, then

$$
F G \odot F^{\prime} G^{\prime}=\left(F \odot F^{\prime}\right)\left(G \odot G^{\prime}\right), \quad \text { in particular } \quad F \odot G=F G ;
$$

(iii) If $X=Y=\{m\}$, then

$$
(F \odot G)(\lambda)=\partial(f * g)\left(r_{m}(\lambda)\right),
$$

where $f$ and $g$ are such that $F(\lambda)=f\left(r_{m}(\lambda)\right), G(\lambda)=g\left(r_{m}(\lambda)\right)$.

Proof. By Proposition 4.4.3, we have

$$
\left(F \odot F^{\prime}\right)(\lambda)=\sum_{\alpha \cup \beta \cup \gamma=\lambda}(-1)^{\ell(\gamma)} F(\alpha) F^{\prime}(\beta),
$$

where it is understood that $\gamma$ is a strict partition. We have that

$$
\begin{aligned}
\left(F \odot F^{\prime}\right)(\lambda) & =\left(\sum_{\alpha \cup \beta \cup \gamma=\left.\lambda\right|_{X}}(-1)^{\ell(\gamma)} F(\alpha) F^{\prime}(\beta)\right)\left(\sum_{\alpha \cup \beta \cup \gamma=\left.\lambda\right|_{X^{c}}}(-1)^{\ell(\gamma)}\right) \\
& =\left(F \odot F^{\prime}\right)\left(\left.\lambda\right|_{X}\right) \cdot(1 \odot 1)\left(\left.\lambda\right|_{X^{c}}\right) .
\end{aligned}
$$

Recall $f \odot 1=f$ for all functions $f$, hence $\left(F \odot F^{\prime}\right)(\lambda)=\left(F \odot F^{\prime}\right)\left(\left.\lambda\right|_{X}\right)$, which is the first statement. 
Next, we have that

$$
\left(F G \odot F^{\prime} G^{\prime}\right)(\lambda)=\sum_{\alpha \cup \beta \cup \gamma=\lambda}(-1)^{\ell(\gamma)}(F G)(\alpha)\left(F^{\prime} G^{\prime}\right)(\beta),
$$

where again it is understood that $\gamma$ is a strict partition. Using the fact that $F, F^{\prime}, G$ and $G^{\prime}$ are supported on $X, X, Y$ and $Y$, respectively, we find that $\left(F G \odot F^{\prime} G^{\prime}\right)(\lambda)$ equals

$$
\sum_{\alpha \cup \beta \cup \gamma=\lambda}(-1)^{\ell\left(\left.\gamma\right|_{X}\right)+\ell\left(\left.\gamma\right|_{Y}\right)+\ell\left(\left.\gamma\right|_{Z}\right)} F\left(\left.\alpha\right|_{X}\right) G\left(\left.\alpha\right|_{Y}\right) F^{\prime}\left(\left.\beta\right|_{X}\right) G^{\prime}\left(\left.\beta\right|_{Y}\right),
$$

where $Z$ denotes the complement of $X \cup Y$ in $\mathbb{N}$. We factor the right-hand side of $(4.25)$ as

$$
\begin{array}{r}
\left(\sum_{\alpha \cup \beta \cup \gamma=\left.\lambda\right|_{X}}(-1)^{\ell(\gamma)} F(\alpha) F^{\prime}(\beta)\right)\left(\sum_{\alpha \cup \beta \cup \gamma=\left.\lambda\right|_{Y}}(-1)^{\ell(\gamma)} G(\alpha) G^{\prime}(\beta)\right) . \\
\left(\sum_{\alpha \cup \beta \cup \gamma=\left.\lambda\right|_{Z}}(-1)^{\ell(\gamma)}\right) .
\end{array}
$$

By definition of the product $\odot$, we conclude

$$
\begin{aligned}
\left(F G \odot F^{\prime} G^{\prime}\right)(\lambda) & =\left(F \odot F^{\prime}\right)\left(\left.\lambda\right|_{X}\right)\left(G \odot G^{\prime}\right)\left(\left.\lambda\right|_{Y}\right)(1 \odot 1)\left(\left.\lambda\right|_{Z}\right) \\
& =\left(F \odot F^{\prime}\right)(\lambda)\left(G \odot G^{\prime}\right)(\lambda) .
\end{aligned}
$$

By taking $F^{\prime}$ and $G$ to be the constant function 1 (which is supported on every $X$ and $Y$ ), we see that $F \odot G^{\prime}=F G^{\prime}$ is implied by $F G \odot F^{\prime} G^{\prime}=\left(F \odot F^{\prime}\right)\left(G \odot G^{\prime}\right)$.

Next, for (iii) we have

$$
\begin{aligned}
(F \odot G)(\lambda) & =\sum_{\alpha \cup \beta \cup \gamma=\lambda}(-1)^{\ell(\gamma)} f\left(r_{m}(\alpha)\right) g\left(r_{m}(\beta)\right) \\
& =\sum_{\alpha \cup \beta \cup \gamma=\left.\lambda\right|_{\{m\}}}(-1)^{\ell(\gamma)} f\left(r_{m}(\alpha)\right) g\left(r_{m}(\beta)\right)
\end{aligned}
$$

Letting $i=r_{m}(\alpha)$ and $j=r_{m}(\beta)$, we have

$$
\begin{aligned}
(F \odot G)(\lambda) & =\sum_{i+j=r_{m}(\lambda)} f(i) g(j)-\sum_{i+j+1=r_{m}(\lambda)} f(i) g(j) \\
& =(f * g)\left(r_{m}(\lambda)\right)-(f * g)\left(r_{m}(\lambda)-1\right) \\
& =\partial(f * g)\left(r_{m}(\lambda)\right) .
\end{aligned}
$$


The second lemma is concerned with the vanishing of certain sums of the Möbius functions of set partitions. Given $\alpha \in \Pi(n)$ and a subset $Z$ of $[n]$, we let

$$
\left.\alpha\right|_{Z}=\{A \cap Z \mid A \in \alpha \text { s.t. } A \cap Z \neq \emptyset\} \in \Pi(Z),
$$

where $\Pi(Z)$ denotes the set of all partitions of the set $Z$. Observe that

$$
\ell(\alpha)=\ell\left(\left.\alpha\right|_{Z}\right)+|\{A \in \alpha \mid A \cap Z=\emptyset\}|,
$$

in particular $\ell\left(\left.\alpha\right|_{Z}\right) \leq \ell(\alpha)$. Given $Z \subset[n]$, define an equivalence relation on $\Pi(n)$ by writing $\alpha \sim \beta$ if

$$
\left.\alpha\right|_{Z}=\left.\beta\right|_{Z} \quad \text { and }\left.\quad \alpha\right|_{Z^{c}}=\left.\beta\right|_{Z^{c}} .
$$

Lemma 4.7.3. Let $Z \subseteq[n]$. If $Z \neq \emptyset$ and $Z \neq[n]$, then for all $\beta \in \Pi(n)$ we have

$$
\sum_{\alpha \sim \beta} \mu(\alpha, \mathbf{1})=0 .
$$

Proof. Observe that $\alpha \sim \beta$ precisely if for all $A \in \alpha$ we have $(A \cap Z=\emptyset$ or $A \cap Z \in$ $\left.\left.\beta\right|_{Z}\right)$ and similarly we have $\left(A \cap Z^{c}=\emptyset\right.$ or $\left.\left.A \cap Z^{c} \in \beta\right|_{Z^{c}}\right)$. Hence, every $A \in \alpha$ is the union of some $\left.A_{1} \in \alpha\right|_{Z} \cup\{\emptyset\}$ and $\left.A_{2} \in \alpha\right|_{Z^{c}} \cup\{\emptyset\}$ with not both $A_{1}=\emptyset$ and $A_{2}=\emptyset$. Write $a=\ell\left(\left.\beta\right|_{Z}\right), b=\ell\left(\left.\beta\right|_{Z^{c}}\right)$, and assume without loss of generality that $a \leq b$. Write $k$ for the number of $A \in \alpha$ for which both $A_{1} \neq \emptyset$ and $A_{2} \neq \emptyset$. Now, $\ell(\alpha)=a+b-k$. Moreover, given $k, Z$ and $\beta$, there are

$$
\left(\begin{array}{l}
a \\
k
\end{array}\right)\left(\begin{array}{l}
b \\
k
\end{array}\right) k !
$$

ways to choose $\alpha \sim \beta$ with $\ell(\alpha)=a+b-k$. Hence, we find

$$
\begin{aligned}
\sum_{\alpha \sim \beta} \mu(\alpha, \mathbf{1}) & =\sum_{k=0}^{a}(-1)^{a+b-k-1}(a+b-k-1) !\left(\begin{array}{l}
a \\
k
\end{array}\right)\left(\begin{array}{l}
b \\
k
\end{array}\right) k ! \\
& =(-1)^{a+b-1}(a+b-1) ! \sum_{k=0}^{a} \frac{(-a)_{k}(-b)_{k}}{(-a-b+1)_{k}(1)_{k}},
\end{aligned}
$$

where $(d)_{k}=\prod_{i=0}^{k-1}(d+i)$ is the rising Pochhammer symbol. This expression equals up to the constant $(-1)^{a+b-1}(a+b-1)$ ! the special value ${ }_{2} F_{1}(-a,-b,-a-b+$ $1 ; 1)$ of the hypergeometric function ${ }_{2} F_{1}(-a,-b,-a-b+1 ; z)$, which vanishes by Gauss's theorem subject to $a, b>0$. As $Z \neq \emptyset$, we have $a>0$. Also, $b>0$ as $Z \neq[n]$. 
The following result not only computes the connected product of the moment functions $S_{k}$, but also is one of the main technical results needed to prove Theorem 4.1.2.

Theorem 4.7.4. Let $k_{i}, f_{i}$ for $i=1, \ldots, n$ be such that (4.11) defines $S_{k_{i}, f_{i}}$. Then

(i) There exists a function $g: \mathbb{N} \rightarrow \mathbb{Q}$ such that

$$
S_{k_{1}, f_{1}}|\ldots| S_{k_{n}, f_{n}}=S_{|\boldsymbol{k}|, g} .
$$

In fact,

$$
g=\sum_{\alpha \in \Pi(n)} \mu(\alpha, \mathbf{1}) \partial^{\ell(\alpha)-1} \underset{A \in \alpha}{*} f_{A},
$$

where $f_{A}=\prod_{a \in A} f_{a}$ and $*$ denotes the convolution product (4.4).

(ii) If $f_{1}(x)=x$, then $\partial g=f_{1} \partial \tilde{g}$ with $\tilde{g}$ given by $S_{k_{2}, f_{2}}|\ldots| S_{k_{n}, f_{n}}=S_{|\boldsymbol{k}|, \tilde{g}}$.

Remark 4.7.5. We extend $g$ by $g(0)=0$. Here and later in this chapter, we usually omit the dependence of $g$ on $f_{1}, \ldots, f_{n}$ in the notation.

Proof. For the first part, we let $\boldsymbol{m}^{\boldsymbol{k}_{\boldsymbol{A}}} \boldsymbol{f}_{\boldsymbol{A}} \circ r_{\boldsymbol{m}}$ denote $\prod_{i} m_{i}^{k_{A_{i}}} \cdot f_{A_{i}} \circ r_{m_{i}}$, where $r_{m_{i}}$ is considered as a function $\mathscr{P} \rightarrow \mathbb{Q}$. In case $n=1$ the result (i) is trivially true, so we assume $n \geq 2$. By definition of the connected product and $S_{k, f}$ (see (4.16) and (4.11), respectively), we have

$$
\begin{aligned}
S_{k_{1}, f_{1}}|\ldots| S_{k_{n}, f_{n}} & =\sum_{\alpha \in \Pi(n)} \mu(\alpha, \mathbf{1}) \bigodot_{A \in \alpha}\left(\sum_{\boldsymbol{m} \in \mathbb{N}^{\ell}(A)} \boldsymbol{m}^{\boldsymbol{k}_{\boldsymbol{A}}} \boldsymbol{f}_{\boldsymbol{A}} \circ r_{\boldsymbol{m}}\right) \\
& =\sum_{\boldsymbol{m} \in \mathbb{N}^{n}} \sum_{\alpha \in \Pi(n)} \mu(\alpha, \mathbf{1}) \bigodot_{A \in \alpha} \boldsymbol{m}_{\boldsymbol{A}}^{\boldsymbol{k}_{\boldsymbol{A}}} \boldsymbol{f}_{\boldsymbol{A}} \circ r_{\boldsymbol{m}} .
\end{aligned}
$$

For all $m \geq 0$, the function $r_{m}: \mathscr{P} \rightarrow \mathbb{Q}$ is supported on $\{m\}$. Having Lemma 4.7.2 in mind, we aim to factor the functions in (4.27) as a product of functions supported on a singleton set. Given $\boldsymbol{m} \in \mathbb{N}^{n}$, we start by all functions supported on $\left\{m_{1}\right\}$, that is, we let $Z(\boldsymbol{m})=\left\{i \mid m_{i}=m_{1}\right\} \subset[n]$. Note that $Z(\boldsymbol{m})$ determines all $i$ for which the support of $r_{m_{i}}$ contains $m_{1}$. Denote by $E(\boldsymbol{m})$ the set of equivalence classes of $\Pi(n)$ for this choice of $Z=Z(\boldsymbol{m})$. We split the sum over $\alpha \in \Pi(n)$ in (4.27) as a sum over the elements of $E(\boldsymbol{m})$, i.e.,

$$
S_{k_{1}, f_{1}}|\ldots| S_{k_{n}, f_{n}}=\sum_{\boldsymbol{m} \in \mathbb{N}^{n}} \sum_{[\beta] \in E(\boldsymbol{m})} \sum_{\alpha \in[\beta]} \mu(\alpha, \mathbf{1}) \bigodot_{A \in \alpha} \boldsymbol{m}_{\boldsymbol{A}}^{\boldsymbol{k}_{\boldsymbol{A}}} \boldsymbol{f}_{\boldsymbol{A}} \circ r_{\boldsymbol{m}_{\boldsymbol{A}}} .
$$


Given $\boldsymbol{m} \in \mathbb{N}^{n}, Z=Z(\boldsymbol{m})$ and $\left.A \in \alpha\right|_{Z}$, the function $\lambda \mapsto \boldsymbol{m}_{\boldsymbol{A}}^{\boldsymbol{k}_{\boldsymbol{A}}} \boldsymbol{f}_{\boldsymbol{A}}\left(r_{\boldsymbol{m}_{\boldsymbol{A}}}(\lambda)\right)$ is supported on $\left\{m_{1}\right\}$, whereas for $\left.A \in \alpha\right|_{Z^{c}}$ the same function is supported on the set $\mathbb{N} \backslash\left\{m_{1}\right\}$. Hence, by Lemma 4.7.2(ii) we find that (4.28) equals

$$
\sum_{\boldsymbol{m} \in \mathbb{N}^{n}} \sum_{[\beta] \in E(\boldsymbol{m})} \sum_{\alpha \in[\beta]} \mu(\alpha, \mathbf{1})\left(\bigodot_{\left.A \in \alpha\right|_{Z}} \boldsymbol{m}_{\boldsymbol{A}}^{\boldsymbol{k}_{\boldsymbol{A}}} \boldsymbol{f}_{\boldsymbol{A}} \circ r_{\boldsymbol{m}_{\boldsymbol{A}}}\right)\left(\bigodot_{\left.A \in \alpha\right|_{Z^{c}}} \boldsymbol{m}_{\boldsymbol{A}}^{\boldsymbol{k}_{\boldsymbol{A}}} \boldsymbol{f}_{\boldsymbol{A}} \circ r_{\boldsymbol{m}_{\boldsymbol{A}}}\right) \text {. }
$$

Instead of writing the second factor as a product of functions which are all supported on a singleton set, we make the following observation. As $\left.\alpha\right|_{Z}=\left.\beta\right|_{Z}$ and $\left.\alpha\right|_{Z^{c}}=\left.\beta\right|_{Z^{c}}$, the only dependence on $\alpha$ in the above equation is in $\mu(\alpha, \mathbf{1})$. By construction $Z(\boldsymbol{m})$ is non-empty. Hence, by Lemma 4.7.3 we have that if $Z \neq[n]$ then for all $\beta \in E(\boldsymbol{m})$ we have $\sum_{\alpha \in[\beta]} \mu(\alpha, \mathbf{1})=0$. This implies that we can restrict the first sum in (4.29) to $\boldsymbol{m} \in \mathbb{N}^{n}$ for which $m_{i}=m_{j}$ for all $i, j$, that is

$$
S_{k_{1}, f_{1}}|\ldots| S_{k_{n}, f_{n}}=\sum_{m \in \mathbb{N}} \sum_{\alpha \in \Pi(n)} \mu(\alpha, \mathbf{1}) \bigodot_{A \in \alpha} \prod_{a \in A} m^{k_{a}} \cdot f_{a} \circ r_{m} .
$$

Applying Lemma 4.7.2(iii) $\ell(\alpha)-1$ times and using (4.5), we obtain the desired result.

For the second part, let $Z=\{1\}$ and consider an equivalence class $[\beta]$ for the equivalence relation (4.26) determined by $Z$. We split the sum

$$
\partial g=\sum_{\alpha \in \Pi(n)} \mu(\alpha, \mathbf{1}) \partial^{\ell(\alpha)} \underset{A \in \alpha}{*} f_{A}
$$

over all conjugacy classes. Write $A_{1}$ for the element of $\alpha$ for which $1 \in A_{1}$. Denote $\hat{A}_{1}=A_{1} \backslash\{1\}$ and $\gamma=\left.\beta\right|_{\{2, \ldots, n\}}$. In case $A_{1}=\{1\}$ one has by (4.8) that

$$
\mu(\alpha, \mathbf{1}) \partial^{\ell(\alpha)} \underset{A \in \alpha}{*} f_{A}=-\ell(\gamma) \mu(\gamma, \mathbf{1}) \partial^{\ell(\gamma)-1}(1-\partial) \underset{A \in \gamma}{*} f_{A} .
$$

In case $A_{1} \neq\{1\}$ (i.e., $\left|A_{1}\right| \geq 2$ ), one finds by (4.6) that

$$
\mu(\alpha, \mathbf{1}) \partial^{\ell(\alpha)} \underset{A \in \alpha}{*} f_{A}=\mu(\gamma, \mathbf{1}) \partial^{\ell(\gamma)-1}\left(f_{1} \partial f_{\hat{A}_{1}}+(1-\partial) f_{\hat{A}_{1}}\right) * \underset{A \in \gamma \backslash \hat{A}_{1}}{*} f_{A} .
$$

As $[\beta]$ contains one element for which (4.30) holds and $\ell(\gamma)$ different elements for which (4.31) holds, one finds

$$
\sum_{\alpha \in[\beta]} \mu(\alpha, \mathbf{1}) \partial^{\ell(\alpha)} \underset{A \in \alpha}{*} f_{A}=\mu(\gamma, \mathbf{1}) \partial^{\ell(\gamma)-1} \sum_{C \in \gamma}\left(f_{1} \partial f_{C} * \underset{A \in \gamma \backslash C}{*} f_{A}\right) .
$$


By (4.5) and (4.7) this equals

$$
\mu(\gamma, \mathbf{1}) \sum_{C \in \gamma}\left(f_{1} \partial f_{C} * \underset{A \in \gamma \backslash C}{*} \partial f_{A}\right)=\mu(\gamma, \mathbf{1}) f_{1} \partial^{\ell(\gamma)} \underset{A \in \gamma}{*} f_{A} .
$$

Hence, summing over all conjugacy classes, we obtain

$$
\partial g=f_{1} \sum_{\gamma \in \Pi(n-1)} \mu(\gamma, \mathbf{1}) \partial^{\ell(\gamma)} \underset{A \in \gamma}{*} f_{A}=f_{1} \partial \tilde{g} .
$$

The case when $f_{1}(x)=\ldots=f_{n}(x)=x$ is the easiest example (for arbitrary $n \in \mathbb{N}$ ) of the above result. In this case one generalises Theorem 4.5.1 by a result which, in accordance with our main principle of establishing identities before the $q$ bracket, yields this theorem after taking the $q$-bracket.

Corollary 4.7.6. For all positive even $k_{1}, \ldots, k_{n}$, one has

$$
S_{k_{1}}|\ldots| S_{k_{n}}=S_{|\boldsymbol{k}|-n, \mathcal{F}_{n}} .
$$

Proof. Recall $S_{k}=S_{k-1 \text {,id }}$ and apply Theorem 4.7.4(ii) $n-1$ times.

Later we will use Theorem 4.5.1 when the $f_{i}$ are Seki-Bernoulli polynomials. This is the situation in which we prove the main result of this chapter, in which case the following lemma is useful.

Lemma 4.7.7. If $f_{1}, \ldots, f_{n}$ are Seki-Bernoulli polynomials of degrees $d_{1}, \ldots, d_{n}$, respectively, and $g: \mathbb{N} \rightarrow \mathbb{Q}$ is as in Theorem 4.7.4, then there exists a polynomial $p$ such that $\partial g(m)=p(m)$ for all $m \in \mathbb{N}$. Moreover, $p$ is strictly of degree $|\boldsymbol{d}|-1$, is even or odd and $p(0)=0$.

Proof. By Theorem 4.7.4(ii) we can assume w.l.o.g. that none of the degrees $d_{i}$ equals 1 . Now, consider a monomial $\partial^{\ell(\alpha)} *_{A \in \alpha} f_{A}$ in $\partial g$. Note that both $*$ and $\partial$ are operators on the space of polynomials, more precisely:

$$
*: \mathbb{Q}[x]_{\leq k} \times \mathbb{Q}[x]_{\leq l} \rightarrow \mathbb{Q}[x]_{\leq k+l+1} \quad \text { and } \quad \partial: \mathbb{Q}[x]_{\leq k} \rightarrow \mathbb{Q}[x]_{\leq k-1}
$$

as

$$
x^{k} * x^{l}=\frac{k ! l !}{(k+l+1) !} x^{k+l+1}+O\left(x^{k+l}\right) \quad \text { and } \quad \partial\left(x^{k}\right)=k x^{k-1}+O\left(x^{k-2}\right) .
$$

Hence the degree of such a monomial is $|\boldsymbol{d}|-1$. Now observe that by the symmetry (4.9) one has

$$
\partial f_{A}(x)=f_{A}(x)-f_{A}(x-1)=f_{A}(x)-(-1)^{|A|} f_{A}(-x) .
$$


Therefore, we see that $\partial f_{A}$ is even or odd and as the convolution product preserves this property, every monomial is even or odd. By the same arguments $\partial f_{A}(0)=0$ and hence the constant term of every monomial vanishes. Therefore, every monomial $\partial^{\ell(\alpha)-1} *_{A \in \alpha} f_{A}$ in $g$ satisfies the desired properties, so that it remains to show that the leading coefficient does not vanish.

As $\mathcal{F}_{l}=\frac{1}{l} x^{l}+O\left(x^{l-1}\right)$, the leading coefficient of a monomial as above equals

$$
\frac{|\boldsymbol{d}|}{\prod_{i} d_{i}} \frac{\prod_{i=1}^{n} d_{A_{i}} !}{|\boldsymbol{d}| !}
$$

where for a set $B$ we have set $d_{B}=\sum_{b \in B} d_{b}$. Hence, the leading coefficient of $\partial g$ equals

$$
\frac{|\boldsymbol{d}|}{\prod_{i} d_{i}} \cdot \sum_{\alpha \in \Pi(n)} \mu(\alpha, \mathbf{1})\left(\begin{array}{c}
|\boldsymbol{d}| \\
d_{A_{1}}, \ldots, d_{A_{r}}
\end{array}\right)^{-1},
$$

where $\alpha=\left\{A_{1}, \ldots, A_{r}\right\}$. Note that this number has the following combinatorial interpretation. Let $n$ balls be given which are coloured such that $d_{1}$ balls are coloured in the first color, $d_{2}$ in the second color, etc. Suppose we use the same multiset of colors to additionally mark each ball with a dot (possibly of the same color), that is, $d_{1}$ balls are marked with a dot of the first color, $d_{2}$ with a dot of the second color, etc. Given a subset $C$ of the set of all colors, it may happen that if we consider all balls coloured by the colors of $C$, all the dots on these balls are coloured by the same set of colors $C$. We then say that the balls are well-coloured with respect to $C$. For example, both the empty set of colors and the set of all possible colors give rise to a well-colouring of balls. If we independently at random color and mark the balls as above, the probability that the balls coloured by a subset $C$ are well-coloured is $\left(\begin{array}{c}|\boldsymbol{d}| \\ d_{C}\end{array}\right)^{-1}$. Hence, by applying Möbius inversion the number

$$
\sum_{\alpha \in \Pi(n)} \mu(\alpha, \mathbf{1})\left(\begin{array}{c}
|\boldsymbol{d}| \\
d_{A_{1}}, \ldots, d_{A_{r}}
\end{array}\right)^{-1}
$$

equals the probability that if we independently at random color and mark the balls as above, there does not exist a proper non-empty subset $C$ of the colors such that the balls coloured by $C$ are well-coloured. If we mark at least one ball of every color $i$ with color $i+1$ (modulo $n$ ), such a set $C$ cannot exist. Hence, the number (4.32) is positive, so the polynomial $p$ is strictly of degree $|\boldsymbol{d}|-1$. 


\subsection{Three quasimodular algebras}

\section{Introduction}

Given integers $k, l$ with $k \geq 0$ and $l \geq 1$ recall the definition of the double moment functions in (4.2) by

$$
T_{k, l}(\lambda)=-\frac{B_{k+l}}{2(k+l)}\left(\delta_{l, 1}+\delta_{k, 0}\right)+\sum_{m=1}^{\infty} m^{k} \mathcal{F}_{l}\left(r_{m}(\lambda)\right) .
$$

Unless stated explicitly, we always assume that

$$
k \in \mathbb{Z}_{\geq 0}, l \in \mathbb{Z}_{\geq 1}, k+l \in 2 \mathbb{Z} .
$$

Moreover, it turns out to be useful to define $T_{0,0} \equiv T_{-1,1} \equiv-1$ and $T_{k, l} \equiv 0$ for other pairs $(k, l)$ with $k<0$ or $l<1$.

Remark 4.8.1. The double moment functions specialise to the moment functions studied in the previous section whenever $l=1$, i.e., $T_{k, 1}=S_{k+1}$. Also, as $\mathcal{F}_{l}(1)=1$, for a strict partition $\lambda$ one has $T_{k, l}(\lambda)=S_{k}(\lambda)$. Hence, our functions $T_{k, l}$ can be seen as an extension of the algebra of supersymmetric polynomials, mentioned in the introduction, to functions on all partitions (and not only on strict partitions).

Remark 4.8.2. In case $k+l$ is odd, the $q$-bracket of $T_{k, l}$ does not vanish-in contrast to the shifted symmetric functions for which the $q$-bracket vanishes for all odd weights. However, the $q$-bracket of a polynomial involving the double moment functions in both even and odd weights also is a polynomial in the so-called combinatorial Eisenstein series, defined in Definition 4.12.6.

These double moment functions give rise to three different graded algebras, which turn out to be quasimodular (see Definition 4.1.1).

Definition 4.8.3. Define the $\mathbb{Q}$-algebras $\mathcal{S}, \operatorname{Sym}^{\odot}(\mathcal{S})$ and $\mathcal{T}$ by the condition that

- $\mathcal{S}$ is generated by the moment functions $S_{k}$ under the pointwise product;

- $\operatorname{Sym}^{\odot}(\mathcal{S})$ is generated by the elements of $\mathcal{S}$ under the induced product;

- $\mathcal{T}$ is generated by the double moment functions under the pointwise product.

Our main result Theorem 4.1.2 is slightly refined by the following statement.

Theorem 4.8.4. Let $X$ be any of the algebras $\mathcal{S}, \operatorname{Sym}^{\odot}(\mathcal{S})$ and $\mathcal{T}$. Then, $X$ is

- quasimodular;

- closed under the pointwise product; 
- closed under the induced product if $X \neq \mathcal{S}$.

Moreover, the three algebras are related by $\mathcal{S} \subsetneq \operatorname{Sym}^{\odot}(\mathcal{S}) \subsetneq \mathcal{T}$.

Remark 4.8.5. Observe that being closed under the pointwise product is not implied by being quasimodular. For example, the algebra $\mathcal{R}=\langle\cdot\rangle_{q}^{-1}(\widetilde{M})$ in Remark 4.4.6 is quasimodular, closed under the induced product and $\mathcal{T} \subset \mathcal{R}$, but $\mathcal{R}$ is not closed under the pointwise product [Zag16, Section 9].

In the next section we provide different bases for these algebras: in this way we obtain many examples of functions with a quasimodular $q$-bracket, and moreover, the study of these bases leads to a proof of Theorem 4.8.4.

Remark 4.8.6. The algebras $\mathcal{T}$ and $\Lambda^{*}$ are different algebras, as follows from the observation that $f(\lambda)=(-1)^{k} f\left(\lambda^{\prime}\right)$ for all $f \in \Lambda_{k}^{*}$ (see (1.11)). This does not hold for all $f \in \mathcal{T}$, as can easily be checked numerically. On the other hand, it is not true that $f(\lambda) \neq \pm f\left(\lambda^{\prime}\right)$ for all $f \in \mathcal{T}$, as $Q_{2}=T_{1,1}$. More precisely, one has

$$
\mathcal{T} \cap \Lambda^{*}=\mathbb{Q}\left[Q_{2}\right] .
$$

Namely, if $f \in \mathcal{T} \cap \Lambda^{*}$, consider a strict partition $\lambda$ (i.e., a partition for which $r_{m}(\lambda) \leq 1$ for all $m$ ). Then, we have that $f(\lambda)$ is symmetric polynomial in the parts $\lambda_{1}, \lambda_{2}, \ldots$. On the other hand, as $f \in \Lambda^{*}$, it follows that $f(\lambda)$ is a shifted symmetric polynomial in the parts $\lambda_{1}, \lambda_{2}, \ldots$. The only polynomials of degree $d$ in the variables $x_{i}$ that are both symmetric and shifted symmetric are up to a constant given by $\left(\sum_{i} x_{i}\right)^{d}$, hence $f \in \mathbb{Q}\left[Q_{2}\right]$.

\section{The basis given by double moment functions}

We show that $\mathcal{T}$ is closed under the induced product. Moreover, we show that $\mathcal{S}$ and $\operatorname{Sym}^{\odot}(\mathcal{S})$ are subalgebras of $\mathcal{T}$. In the next section, we use these results to define a weight grading on $\mathcal{T}$. Observe that as a vector space $\mathcal{T}$ is spanned by the functions $T_{\boldsymbol{k}, \boldsymbol{l}}$, defined by $T_{\boldsymbol{k}, \boldsymbol{l}}=\prod_{i} T_{k_{i}, l_{i}}$, for all $\boldsymbol{k}, \boldsymbol{l} \in \mathbb{Z}^{n}$ satisfying the conditions (4.33) for all pairs $(k, l)=\left(k_{i}, l_{i}\right)$.

Theorem 4.8.7. The algebra $\mathcal{T}$ is closed under the induced product.

Proof. Observe that

$$
T_{\boldsymbol{k}, \boldsymbol{l}} \odot T_{\boldsymbol{k}^{\prime}, \boldsymbol{l}^{\prime}}=T_{\boldsymbol{k}, \boldsymbol{l}} T_{\boldsymbol{k}^{\prime}, \boldsymbol{l}^{\prime}}-T_{\boldsymbol{k}, \boldsymbol{l}} \mid T_{\boldsymbol{k}^{\prime}, l^{\prime}} .
$$

Hence, it suffices to show that $T_{k, l} \mid T_{\boldsymbol{k}^{\prime}, l^{\prime}}$ can be expressed in terms of elements of $\mathcal{T}$.

By Theorem 4.7.4 and Lemma 4.7.7, we have that an expression of the form

$$
T_{k_{1}, l_{1}}|\cdots| T_{k_{n}, l_{n}}
$$


as an element of $\mathcal{T}$. Proposition 4.4.7 implies that $f_{1} f_{2}\left|f_{3}\right| f_{4}|\ldots| f_{n}$ equals

$$
\begin{aligned}
\left(f_{1}\left|f_{2}\right| \ldots \mid f_{n}\right)+\sum_{A \sqcup B=\{3, \ldots, n\}}\left(\left(f_{1}\left|f_{A_{1}}\right| f_{A_{2}} \mid \ldots\right) \cdot\left(f_{2}\left|f_{B_{1}}\right| f_{B_{2}} \mid \ldots\right)\right. \\
\left.-\left(f_{1}\left|f_{A_{1}}\right| f_{A_{2}} \mid \ldots\right) \mid\left(f_{2}\left|f_{B_{1}}\right| f_{B_{2}} \mid \ldots\right)\right) .
\end{aligned}
$$

Hence, by using this proposition recursively, we can replace the pointwise products in $T_{\boldsymbol{k}, l} \mid T_{\boldsymbol{k}^{\prime}, l^{\prime}}$ by a linear combination of connected products of double moment functions $T_{k, l}$, showing that $T_{\boldsymbol{k}, l} \mid T_{\boldsymbol{k}^{\prime}, \boldsymbol{l}^{\prime}}$ is an element of $\mathcal{T}$.

Now, we determine a basis for the three algebras. Let $\mathcal{T}^{\text {mon }}$ be the set of all monomials for the pointwise product in $\mathcal{T}$. Two elements of $\mathcal{T}^{\text {mon }}$ are considered to be the same if one can reorder the products so that they agree, for example $T_{1,1} T_{3,5}$ and $T_{3,5} T_{1,1}$ are the same function. In other words: every elements of $\mathcal{T}^{\text {mon }}$ can be written as $T_{k, l}$ in a unique way up to commutativity of the (pointwise) product.

Theorem 4.8.8. We have

$$
\mathcal{S} \subsetneq \operatorname{Sym}^{\odot}(\mathcal{S}) \subsetneq \mathcal{T}
$$

Moreover, a basis for

- $\mathcal{T}$ is given by $\mathcal{T}^{\mathrm{mon}}$;

- $\operatorname{Sym}^{\odot}(\mathcal{S})$ is given by all $T_{\boldsymbol{k}, \boldsymbol{l}} \in \mathcal{T}^{\mathrm{mon}}$ satisfying $k_{i} \geq l_{i}$ for all $i$;

- $\mathcal{S}$ is given by all $T_{\boldsymbol{k}, \boldsymbol{l}} \in \mathcal{T}^{\text {mon }}$ satisfying $l_{i}=1$ for all $i$.

Proof. It suffices to prove the second part, as from the stated bases statement (4.34) follows immediately.

By definition the elements of $\mathcal{T}^{\text {mon }}$ generate $\mathcal{T}$ as a vector space. Hence, it suffices to show that they are linearly independent, i.e., that if

$$
\sum_{\alpha \in I} c_{\alpha} T_{\alpha}(\lambda)=0
$$

for all $\lambda \in \mathscr{P}$, where $I$ is the set of all pairs $(\boldsymbol{k}, \boldsymbol{l})$ up to simultaneous reordering and $c_{\alpha} \in \mathbb{Q}$, we have that $c_{\alpha}=0$ for all $\alpha$.

First of all, let $\lambda=\left(N_{1}, N_{2}\right)$ and consider (4.35) as $N_{1} \rightarrow \infty$. Note that $T_{\boldsymbol{k}, \boldsymbol{l}}(\lambda)$ grows as

$$
N_{1}^{|\boldsymbol{k}|}+N_{2}^{k_{\min }} N_{1}^{\left|\boldsymbol{k} \backslash k_{\min }\right|}
$$

plus lower-order terms, where $k_{\min }$ is the smallest of the $k_{i}$ in $\boldsymbol{k}$. Hence, $|\boldsymbol{k}|$ should be constant among all $T_{\alpha}$ in (4.35). Moreover, we conclude that $k_{\min }$ should be constant among all $T_{\alpha}$ in (4.35). Continuing by considering the lower-order terms, we 
conclude that $\boldsymbol{k}$ is constant among all $T_{\alpha}$. Similarly, by instead considering partitions consisting of $N_{1}$ times the part 1 and $N_{2}$ times the part 2, we conclude that $\boldsymbol{l}$ is constant among all $T_{\alpha}$. Hence, there is at most one $\alpha$ with nonzero coefficient $c_{\alpha}$. We conclude that $c_{\alpha}=0$ for all $\alpha \in I$.

For $\operatorname{Sym}^{\odot}(\mathcal{S})$ we show, first of all, that indeed $T_{k, l} \in \operatorname{Sym}^{\odot}(\mathcal{S})$ if $k_{i} \geq l_{i}$ for all $i$. Let $k \geq l$ of the same parity be given. By Corollary 4.7.6 we find that

$$
\underbrace{T_{1,1}\left|T_{1,1}\right| \ldots \mid T_{1,1}}_{l-1}|T_{k-l+1,1}=\underbrace{S_{2}\left|S_{2}\right| \ldots \mid S_{2}}_{l-1}| S_{k-l+2}=T_{k, l} .
$$

Therefore, $T_{k, l} \in \operatorname{Sym}^{\odot}(\mathcal{S})$ for all $k \geq l$. Moreover, by applying Möbius inversion on Equation (4.16), which defines the connected product, we find

$$
T_{\boldsymbol{k}, \boldsymbol{l}}=\sum_{\alpha \in \Pi(n)} \bigodot_{A \in \alpha}\left(T_{k_{A_{1}}, l_{A_{1}}}\left|T_{k_{A_{2}}, l_{A_{1}}}\right| \ldots\right) .
$$

As we already showed that $T_{k, l} \in \operatorname{Sym}^{\odot}(\mathcal{S})$ if $k \geq l$, we find $T_{k, l} \in \operatorname{Sym}^{\odot}(\mathcal{S})$ if $k_{i} \geq l_{i}$ for all $i$.

Next, we show that all elements in $\operatorname{Sym}^{\odot}(\mathcal{S})$ are a linear combination of the $T_{k, l}$ satisfying $k_{i} \geq l_{i}$. As $\mathcal{S}$ clearly is contained in the space generated by the $T_{\boldsymbol{k}, \boldsymbol{l}}$ for which $k_{i} \geq l_{i}$, it suffices to show that the latter space is closed under $\odot$. For this we follow the proof of Theorem 4.8.7 observing that in each step $k_{i} \geq l_{i}$, so that indeed the $T_{\boldsymbol{k}, \boldsymbol{l}}$ for which $k_{i} \geq l_{i}$ form a generating set for $\operatorname{Sym}^{\odot}(\mathcal{S})$.

As we already showed that the $T_{k, l}$ are linearly independent, we conclude that the $T_{\boldsymbol{k}, \boldsymbol{l}} \in \mathcal{T}^{\text {mon }}$ satisfying $k_{i} \geq l_{i}$ for all $i$ form a basis for $\operatorname{Sym}^{\odot}(\mathcal{S})$.

The last part of the statement follows directly, as by definition all $T_{k, l} \in \mathcal{T}^{\text {mon }}$ satisfying $l_{i}=1$ for all $i$ generate $\mathcal{S}$, and by the above they are linearly independent.

\section{The basis defining the weight grading}

By definition, the double moment functions generate $\mathcal{T}$ under the pointwise product. In this section we show that we can replace the pointwise product in the latter statement by the induced product. Again we will consider every reordering of the factors in $T_{k_{1}, l_{1}} \odot \cdots \odot T_{k_{n}, l_{n}}$ due to commutativity of the products to be the same element. Then, we have:

Theorem 4.8.9. The elements $T_{k_{1}, l_{1}} \odot \cdots \odot T_{k_{n}, l_{n}}$ form a basis for $\mathcal{T}$. A basis for the subspace $\operatorname{Sym}^{\odot}(\mathcal{S})$ is given by the subset of elements for which $k_{i} \geq l_{i}$ for all $i$.

Proof. Assign to $T_{k, l}$ weight $k+l$. This defines a weight filtering on $\mathcal{T}$ with respect to the pointwise product. Consider the subspace of elements of weight at most $w$ 
in $\mathcal{T}$. The number of basis elements in the basis given by the pointwise product in the previous section equals the number of induced products of the $T_{k, l}$. Hence, it suffice that the induced products of the $T_{k, l}$ generate $\mathcal{T}$. For this we proceed by induction first on the weight and then on the depth. Here, by depth we mean the unique filtering under the pointwise product for which every $T_{k, l}$ has depth 1 , usually called the total depth.

Trivially, every element of weight 0 or depth 0 is generated by (empty) induced products of the $T_{k, l}$. Next, consider $T_{\boldsymbol{k}, \boldsymbol{l}} \in \mathcal{T}$ and assume all elements of lower weight and of the same weight and lower depth are generated by induced product of the $T_{k, l}$. Let $T_{\boldsymbol{k}, \boldsymbol{l}} \in \mathcal{T}$ of weight $w$ be given and write $\boldsymbol{k}^{\prime}, \boldsymbol{l}^{\prime}$ for $\boldsymbol{k}, \boldsymbol{l}$ after omitting the last ( $n$ th) entry. Then,

$$
T_{\boldsymbol{k}, \boldsymbol{l}}=T_{\boldsymbol{k}^{\prime}, \boldsymbol{l}^{\prime}} \odot T_{k_{n}, l_{n}}-T_{\boldsymbol{k}^{\prime}, \boldsymbol{l}^{\prime}} \mid T_{k_{n}, l_{n}} .
$$

Note that $T_{\boldsymbol{k}^{\prime}, l^{\prime}}$ is of weight strictly less than $w$, hence is generated by induced products of the $T_{k, l}$. Moreover, by Proposition 4.4.7 and Theorem 4.7.4 it follows that the depth of $T_{\boldsymbol{k}^{\prime}, \boldsymbol{l}^{\prime}} \mid T_{k_{n}, l_{n}}$ is at most $n-1$. Hence, by our induction hypothesis, it is generated by induced products of the $T_{k, l}$. We conclude that $T_{\boldsymbol{k}, \boldsymbol{l}}$ is generated by induced products of the $T_{k, l}$, which proves the first part of the theorem.

The second part follows by the same proof, everywhere restricting to those $T_{k, l}$ for which $k \geq l$.

By the above theorem, we can define a weight grading on $\mathcal{T}$.

Definition 4.8.10. Define a weight grading on $\mathcal{T}$ by assigning to $T_{k, l}$ weight $k+l$ and extending under the induced product.

Note that both the grading on $\mathcal{T}$ and the grading on $\mathcal{S}$ correspond to the grading on quasimodular forms after taking the $q$-bracket. Hence, the grading on $\mathcal{S}$ is the restriction of the grading on $\mathcal{T}$.

The weight grading defines a weight operator. In Section 4.10 we extend this weight operator to an $\mathfrak{s l}_{2}$-triple acting on $\mathcal{T}$, so that $\mathcal{T}$ becomes an $\mathfrak{s l}_{2}$-algebra.

\section{The $n$-point functions}

As induced products of the $T_{k, l}$ form a basis for $\mathcal{T}$, knowing $\langle f\rangle_{q}$ for all $f \in \mathcal{T}$ is equivalent to knowing the generating function $G_{n}\left(u_{1}, \ldots u_{n}, v_{1}, \ldots v_{n}\right)$, called the $n$-point function and given by

$$
\sum_{\boldsymbol{k}, \boldsymbol{l}}\left\langle T_{k_{1}, l_{1}} \odot \cdots \odot T_{k_{n}, l_{n}}\right\rangle_{q} \frac{u_{1}^{k_{1}} \cdots u_{n}^{k_{n}} v_{1}^{l_{1}-1} \cdots v_{n}^{l_{n}-1}}{k_{1} ! \cdots k_{n} !\left(l_{1}-1\right) ! \cdots\left(l_{n}-1\right) !}
$$


for all $n \geq 0$. Here the sum is over all $k_{i}, l_{i}$ such that $k_{i}+l_{i}$ is even and $m$ ! is consider to be 1 for $m<0$. As the $q$-bracket is a homomorphism with respect to the induced product, we directly conclude that

$$
G_{n}(\boldsymbol{u}, \boldsymbol{v})=\prod_{i=1}^{n} G_{1}\left(u_{i}, v_{i}\right)
$$

We also define the partition function by

$$
\Phi(\boldsymbol{t})=\sum_{n=0}^{\infty} \frac{1}{n !} \sum_{\boldsymbol{k}, \boldsymbol{l}}\left\langle T_{k_{1}, l_{1}} \odot \cdots \odot T_{k_{n}, l_{n}}\right\rangle_{q} t_{k_{1}, l_{1}} \cdots t_{k_{n}, l_{n}} .
$$

The following result (together with (4.37)) expresses these functions in terms of the Jacobi theta series (1.14).

Theorem 4.8.11. For all $n \geq 0$ one has

$$
\begin{aligned}
G_{1}(u, v) & =-\frac{1}{2} \frac{\theta^{\prime}(0) \theta(u+v)}{\theta(u) \theta(v)}, \\
\Phi(\boldsymbol{t}) & =\exp \left(\left[x^{0} y^{0}\right] G_{1}\left(\frac{\partial}{\partial x}, \frac{\partial}{\partial y}\right) \sum_{k, l} t_{k, l} x^{k} y^{l}\right),
\end{aligned}
$$

where $\left[x^{0} y^{0}\right]$ denotes taking the constant coefficient.

Proof. We have that

$$
G_{1}(u, v)=\prod_{i=1}^{n}\left(-\frac{1}{2 u}-\frac{1}{2 v}+\left(\sum_{k, l} D^{l-1} G_{k-l+2}+\sum_{k, l} D^{k} G_{l-k}\right) \frac{u^{k} v^{l-1}}{k !(l-1) !}\right),
$$

where in the sum it is understood that $k+l$ is even, $k \geq 0, l \geq 1$. The expression for $G_{1}(u, v)$ in the statement now follows from [Zag91, $\left.\S \S 3\right]$. The expression for $\Phi$ follows immediately from this result.

\subsection{The derivative of a function on partitions}

Note that for all $f \in \mathbb{Q}^{\mathscr{P}}$ one has

$$
D\langle f\rangle_{q}=\left\langle S_{2} f\right\rangle_{q}-\left\langle S_{2}\right\rangle_{q}\langle f\rangle_{q} .
$$

Hence, by letting $D f:=S_{2} \mid f=S_{2} f-S_{2} \odot f$ for $f \in \mathbb{Q}^{\mathscr{P}}$, we have that $D\langle f\rangle_{q}=$ $\langle D f\rangle_{q}$. Moreover, $D$ acts as a derivation. 
Proposition 4.9.1. The map $D: \mathbb{Q}^{\mathscr{P}} \rightarrow \mathbb{Q}^{\mathscr{P}}$ is an equivariant derivation, i.e., $D$ is linear, satisfies the Leibniz rule and

$$
D\langle f\rangle_{q}=\langle D f\rangle_{q}
$$

In fact, for all $k \geq 1$, the mapping $f \mapsto S_{k} \mid f$ is a derivation. Recall the definition of the Möbius function $\mu$ defined in Proposition 4.3.3 and denote $S_{k}^{0}=S_{k}-S_{k}(\emptyset)$.

Lemma 4.9.2. For all even $m \geq 2$ one has

(i) $S_{m}^{0} \odot \mu=-S_{m}^{0} \mu$;

(ii) The mapping $\left(\mathbb{Q}^{\mathscr{P}}, \odot\right) \rightarrow\left(\mathbb{Q}^{\mathscr{P}}, \odot\right), f \mapsto S_{m} \mid f$ is a derivation, uniquely determined on $\mathcal{T}$ by

$$
S_{m} \mid T_{k, l}=T_{k+m-1, l+1} .
$$

Remark 4.9.3. In case $m \geq 4$, the derivation $f \mapsto S_{m} \mid f$ does not correspond to a derivation on $\widetilde{M}$, i.e., a derivation $\mathfrak{d}_{m}$ such that $\mathfrak{d}_{m}\langle f\rangle_{q}=\left\langle S_{m} \mid f\right\rangle_{q}$ for all $f \in \mathcal{T}$. For instance, although the $q$-brackets of $T_{m, m}$ and $T_{m-1, m+1}$ are the same, the $q$ brackets of $S_{m} \mid T_{m, m}=T_{2 m-1, m+1}$ and $S_{m} \mid T_{m-1, m+1}=T_{2 m-2, m+2}$ are different.

Proof. First of all, by Proposition 4.3.4, one has

$$
\left(\sum_{\lambda \in \mathscr{P}} u_{\lambda}\right)\left\langle S_{k}^{0} \odot \mu\right\rangle_{\boldsymbol{u}}=\left(\sum_{m, r \geq 1} m^{k-1} u_{m}^{r}\right)\left(\sum_{\lambda \in \mathscr{P}} \mu(\lambda) u_{\lambda}\right) .
$$

Let $\mathscr{S}_{m}$ be the set of strict partitions not containing $m$ as a part. Then, we can rewrite (4.39) as

$$
\sum_{m} \sum_{\lambda \in \mathscr{S}_{m}} m^{k-1} \mu(\lambda) u_{m} u_{\lambda}=-\sum_{\lambda \in \mathscr{P}} S_{k}^{0}(\lambda) \mu(\lambda) u_{\lambda},
$$

since $\mu(\lambda \cup(m))=-\mu(\lambda)$ for $\lambda \in \mathscr{S}_{m}$, so that for $r \geq 2$ the coefficient of $u_{m}^{r} u_{\lambda}$ cancels in pairs. We conclude that $S_{k}^{0} \odot \mu=-S_{k}^{0} \mu$.

For the second part, note that (i) implies that

$$
S_{k} \odot \mu=-\left(S_{k}+\frac{B_{k}}{k}\right) \mu .
$$

Let $f, g \in \mathbb{Q}^{\mathscr{P}}$ be given. Then

$$
S_{k} \mid(f \odot g)=S_{k}(f \odot g)-S_{k} \odot f \odot g .
$$


If $\alpha \cup \beta \cup \gamma=\lambda$ then $S_{k}(\lambda)=S_{k}(\alpha)+S_{k}(\beta)+S_{k}(\gamma)+\frac{B_{k}}{k}$, hence

$$
\begin{aligned}
S_{k}(\lambda) & (f \odot g)(\lambda) \\
& =\sum_{\alpha \cup \beta \cup \gamma=\lambda}\left(S_{k}(\alpha)+S_{k}(\beta)+S_{k}(\gamma)+\frac{B_{k}}{k}\right) f(\alpha) g(\beta) \mu(\gamma) \\
& =\left(S_{k} f\right) \odot g+f \odot\left(S_{k} g\right)+\sum_{\alpha \cup \beta \cup \gamma=\lambda}\left(S_{k}(\gamma)+\frac{B_{k}}{k}\right) f(\alpha) g(\beta) \mu(\gamma) \\
& =\left(S_{k} f\right) \odot g+f \odot\left(S_{k} g\right)-\sum_{\alpha \cup \beta \cup \gamma=\lambda}\left(S_{k} \odot \mu\right)(\gamma) f(\alpha) g(\beta) \\
& =\left(S_{k} f\right) \odot g+f \odot\left(S_{k} g\right)-S_{k} \odot f \odot g .
\end{aligned}
$$

Therefore,

$S_{k} \mid(f \odot g)=\left(S_{k} f\right) \odot g+f \odot\left(S_{k} g\right)-2 S_{k} \odot f \odot g=\left(S_{k} \mid f\right) \odot g+f \odot\left(S_{k} \mid g\right)$,

i.e., the mapping $f \mapsto S_{k} \mid f$ is a derivation. The formula $S_{m} \mid T_{k, l}=T_{k+m-1, l+1}$ follows directly from Theorem 4.7.4.

Proof of Proposition 4.9.1. As $S_{2} \mid f=S_{2} f-S_{2} \odot f$ is derivation by the above lemma, the results follows directly from (4.38).

\subsection{The equivariant $q$-bracket and Rankin-Cohen brackets}

In this section we extend the action by the $\mathfrak{s l}_{2}$-triple $(D, W, \mathfrak{d})$ on quasimodular forms to $\mathcal{T}$. As the derivation $\mathfrak{d}$ does not act on all power series in $q$, but only on quasimodular forms, we cannot hope to define $\mathfrak{d}$ on all functions on partitions as we did with $D$. On the algebra $\mathcal{T}$, however, this is possible. We define an $\mathfrak{s l}_{2}$-action on this space and we show that the $q$-bracket restricted to $\mathcal{T}$ is an equivariant map of $\mathfrak{s l}_{2}$-algebras.

Note that the following definition agrees with the definition of $D$ in the previous section:

Definition 4.10.1. Define the derivations $D, W, \mathfrak{d}$ on $\mathcal{T}$ by

$$
\begin{aligned}
D T_{k, l} & =T_{k+1, l+1}, \\
W T_{k, l} & =(k+l) T_{k, l}, \\
\mathfrak{d} T_{k, l} & =k(l-1) T_{k-1, l-1}-\frac{1}{2} \delta_{k+l-2} .
\end{aligned}
$$

One immediately checks that $D, W$ and $\mathfrak{d}$ satisfy the commutation relation of an $\mathfrak{s l}_{2}$-triple on $\mathcal{T}$. The corresponding acting of $\mathfrak{s l}_{2}$ on $\mathcal{T}$ makes the $q$-bracket equivariant, so that a refined version of Theorem 4.1.3 is: 
Theorem 4.10.2 (The $\mathfrak{s l}_{2}$-equivariant symmetric Bloch-Okounkov theorem). The algebra $\mathcal{T}$ is an $\mathfrak{s l}_{2}$-algebra with respect to the above action of $\mathfrak{s l}_{2}$ on $\mathcal{T}$. Moreover, the q-bracket becomes an equivariant map of $\mathfrak{s l}_{2}$-algebras, i.e., for $f \in \mathcal{T}$ one has

$$
D\langle f\rangle_{q}=\langle D f\rangle_{q}, \quad W\langle f\rangle_{q}=\langle W f\rangle_{q}, \quad \mathfrak{d}\langle f\rangle_{q}=\langle\mathfrak{d} f\rangle_{q} .
$$

Proof. We already observed that the first of the three equality holds and the second is the homogeneity statement. Hence, it suffices to prove the last statement. Observe that by the commutation relations for $\mathfrak{d}$ and $D$, for all $n \geq 1$ one has

$$
\left[\mathfrak{d}, D^{n}\right]=n(W-n+1) D^{n-1} .
$$

Using (4.40) we find that for $a \geq 0, b \geq 2$ one has

$$
\mathfrak{d}\left(D^{a} G_{b}\right)=a(a+b-1) D^{a-1} G_{b}-\frac{1}{2} \delta_{a+b-2} .
$$

Hence,

$$
\mathfrak{d}\left\langle T_{k, l}\right\rangle_{q}=k(l-1)\left\langle T_{k-1, l-1}\right\rangle_{q}-\delta_{k+l-2}=\left\langle\mathfrak{d} T_{k, l}\right\rangle_{q}
$$

and the last statement follows from the Leibniz rule.

The $\mathfrak{s l}_{2}$-action allows us to define Rankin-Cohen brackets on $\mathcal{T}$.

Definition 4.10.3. For two elements $f, g \in \mathcal{T}$ and $n \geq 0$ the $n$th Rankin-Cohen bracket is given by

$$
[f, g]_{n}=\sum_{\substack{r, s \geq 0 \\
r+s=n}}(-1)^{r}\left(\begin{array}{c}
k+n-1 \\
s
\end{array}\right)\left(\begin{array}{c}
l+n-1 \\
r
\end{array}\right) D^{r} f \odot D^{s} g .
$$

Note that the formula (4.41) would have defined the Rankin-Cohen brackets on $\widetilde{M}$ if $D$ acts by $q \frac{\partial}{\partial q}$ and the induced product is replaced by the usual product, whereas in this line $D$ acts on $\mathcal{T}$ as explained in the previous sections.

If $f, g \in \operatorname{ker} \mathfrak{d}$, then $\langle f\rangle_{q}$ and $\langle g\rangle_{q}$ are modular forms. The Rankin-Cohen bracket of two modular forms is a modular form; analogously, we have:

Proposition 4.10.4. If $f, g \in \operatorname{ker} \mathfrak{d}$, then $[f, g]_{n} \in \operatorname{ker} \mathfrak{d}$.

Proof. Using (4.40), we find that

$$
\begin{array}{r}
\mathfrak{d}[f, g]_{n}=\sum_{\substack{r, s \geq 0 \\
r+s=n}}(-1)^{r} \frac{(k+n-1) !}{s !(k+r-2) !} \frac{(l+n-1) !}{(r-1) !(k+s-1) !} D^{r-1} f \odot D^{s} g+ \\
(-1)^{r} \frac{(k+n-1) !}{(s-1) !(k+r-1) !} \frac{(l+n-1) !}{r !(l+s-2) !} D^{r} f \odot D^{s-1} g,
\end{array}
$$

where $\frac{1}{(-1) !}$ should taken to be 0 . This is a telescoping sum, vanishing identically. 
Remark 4.10.5. The above bracket makes the algebra $\mathcal{T}$ into a Rankin-Cohen algebra, meaning the following. Let $A_{*}=\oplus_{k \geq 0} A_{k}$ be a graded $K$-vector space with $A_{0}=K$ and $\operatorname{dim} A_{k}<\infty$ (for us $A=\mathcal{T}$ ). We say $A$ is a Rankin-Cohen algebra if there are bilinear operations $[,]_{n}: A_{k} \otimes A_{l} \rightarrow A_{k+l+2 n}(k, l, n>0)$ which satisfy all the algebraic identities satisfied by the Rankin-Cohen brackets on $\widetilde{M}$ [Zag94].

\section{A restricted $\mathfrak{s l}_{2}$-action}

Theorem 4.10.2 does not make $\mathcal{S}$ into an $\mathfrak{s l}_{2}$-algebra. Namely, $D$ does not preserve $\mathcal{S}$. However, if we allow ourselves to deform the $\mathfrak{s l}_{2}$-triple $(D, W, \mathfrak{d})$ as in Section 2.2, we can define an $\mathfrak{s l}_{2}$-action on $\mathcal{S}$. This action, however, does not make $\mathcal{S}$ into an $\mathfrak{s l}_{2}$ algebra, as the deformed operators are not derivations.

The operator taking the role of $\mathfrak{d}$ is the operator $\mathfrak{s}: \mathcal{S}_{k} \rightarrow \mathcal{S}_{k-2}$ defined by

$$
\mathfrak{s}=\frac{1}{2} \sum_{k, l \geq 0}(k+l) S_{k+l} \frac{\partial^{2}}{\partial S_{k+1} \partial S_{l+1}}-\frac{1}{2} \frac{\partial}{\partial S_{2}} .
$$

The operator $D$ is replaced by multiplication with $S_{2}$.

Lemma 4.10.6. The triple $\left(S_{2}, W-\frac{1}{2}, \mathfrak{s}\right)$ forms an $\mathfrak{s l}_{2}$-triple of operators acting on $\mathcal{S}$.

Proof. Observe that

$$
\left[\mathfrak{s}, S_{2}\right] f=\sum_{k}(k+1) S_{k+1} \frac{\partial}{\partial S_{k+1}} f-\frac{1}{2} f=\left(W-\frac{1}{2}\right) f .
$$

As $\mathfrak{s}$ and $S_{2}$ decrease, respectively, increase the weight by 2 , the claim follows.

Theorem 4.10.7. The q-bracket $\langle\cdot\rangle_{q}: \mathcal{S} \rightarrow \widetilde{M}$ is an equivariant mapping with respect to the $\mathfrak{s l}_{2}$-triple $\left(S_{2}, W-\frac{1}{2}, \mathfrak{s}\right)$ on $\mathcal{S}$ and the $\mathfrak{s l}_{2}$-triple $\left(D+G_{2}, W-\frac{1}{2}, \mathfrak{d}\right)$ on $\widetilde{M}$, i.e., for all $f \in \mathcal{S}$ one has

$$
\left(D+G_{2}\right)\langle f\rangle_{q}=\left\langle S_{2} f\right\rangle_{q}, \quad\left(W-\frac{1}{2}\right)\langle f\rangle_{q}=\left\langle\left(E-\frac{1}{2}\right) f\right\rangle_{q}, \quad \mathfrak{d}\langle f\rangle_{q}=\langle\mathfrak{s} f\rangle_{q} .
$$

Proof. The first of the three equalities in (4.42) follows from the definition of the $q$ bracket; the second is the homogeneity statement of Theorem 4.10.2. Hence, it remains to prove the last equation $\mathfrak{d}\langle f\rangle_{q}=\langle\mathfrak{s} f\rangle_{q}$.

Given $\boldsymbol{k} \in \mathbb{N}^{n}$, let $\boldsymbol{k}^{i} \in \mathbb{N}^{n-1}$ be given by $\boldsymbol{k}^{i}:=\left(k_{1}, \ldots, k_{i-1}, k_{i+1}, \ldots, k_{n}\right)$ omitting $k_{i}$. Similarly, define $\boldsymbol{k}^{i, j} \in \mathbb{N}^{n-2}$ by omitting $k_{i}$ and $k_{j}$. Then

$$
\mathfrak{s} S_{\boldsymbol{k}}=\sum_{i \neq j}\left(k_{i}+k_{j}-2\right) S_{k_{i}+k_{j}-2} S_{\boldsymbol{k}^{i, j}}-\frac{1}{2} \sum_{i: k_{i}=2} S_{\boldsymbol{k}^{i}} .
$$


By Theorem 4.5.1, one finds

$$
\left\langle S_{k_{i}+k_{j}-2} S_{\boldsymbol{k}^{i, j}}\right\rangle_{q}=\sum_{\substack{\beta \in \Pi(n) \\ \exists I \in \beta:\{i, j\} \subset I}} D^{\ell(I)-2} G_{\left|k_{I}\right|-2 \ell(I)+2} \prod_{B \neq I} D^{\ell(A)-1} G_{\left|k_{B}\right|-2 \ell(A)+2} .
$$

For $I \in \beta$ and $l \in \mathbb{N}^{I}$, let

$$
C(I, \boldsymbol{l}):=\sum_{i, j \in I, i \neq j}\left(l_{i}+l_{j}-2\right)=(\ell(I)-1)(|\boldsymbol{l}|-\ell(I)) .
$$

It follows that $\sum_{i \neq j}\left(k_{i}+k_{j}-2\right)\left\langle S_{k_{i}+k_{j}-2} S_{\boldsymbol{k}^{i, j}}\right\rangle_{q}$ equals

$$
\sum_{\beta \in \Pi(n)} \sum_{I \in \beta} 2 C(I, \boldsymbol{k}) D^{\ell(I)-2} G_{\left|k_{I}\right|-2 \ell(I)+2} \prod_{B \neq I} D^{\ell(B)-1} G_{\left|k_{B}\right|-2 \ell(B)+2} .
$$

On the other hand, observe that if $f$ is of weight $|\boldsymbol{l}|-2 \ell(I)+2$, Equation (4.40) yields

$$
\left[\mathfrak{d}, D^{\ell(I)-1}\right] f=C(I, \boldsymbol{l}) D^{\ell(I)-2} f .
$$

Hence, using $\mathfrak{d} G_{k}=-\frac{1}{2} \delta_{k, 2}$, we obtain

$$
\left[\mathfrak{d}, D^{\ell(I)-1}\right] G_{\left|k_{I}\right|-2 \ell(I)+2}=C\left(B, \boldsymbol{k}_{I}\right) D^{\ell(I)-2} G_{\left|k_{I}\right|-2 \ell(I)+2}-\frac{1}{2} \delta_{\boldsymbol{k}_{I},(2)}
$$

Therefore,

$$
\begin{aligned}
\mathfrak{d}\left\langle S_{\boldsymbol{k}}\right\rangle_{q}= & \sum_{\beta \in \Pi(n)} \sum_{I \in \beta} C\left(I, \boldsymbol{k}_{I}\right) D^{\ell(I)-2} G_{\left|k_{I}\right|-2 \ell(I)+2} \prod_{B \neq I} D^{\ell(B)-1} G_{\left|k_{B}\right|-2 \ell(B)+2}+ \\
& -\frac{1}{2} \sum_{i: k_{i}=2} \sum_{\beta \in \Pi([n] \backslash\{i\})} \prod_{B \in \beta} D^{\ell(B)-1} G_{\left|k_{B}\right|-2 \ell(B)+2},
\end{aligned}
$$

which by the above reasoning is exactly equal to $\left\langle\mathfrak{s} S_{\boldsymbol{k}}\right\rangle_{q}$.

\subsection{The structure constants: from the pointwise to the induced product}

\section{The structure constants}

In Theorem 4.7.4, we deduced that $T_{k_{1}, f_{1}}|\ldots| T_{k_{n}, f_{n}}=T_{|\boldsymbol{k}|, g}$ with

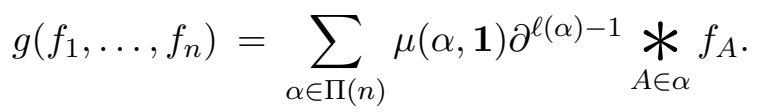


In the particular case that $f_{1}=\ldots=f_{n}$ is the identity function, we saw in Corollary 4.7.6 that $g=\mathcal{F}_{n}$. If $f_{1}, \ldots, f_{n}$ are Seki-Bernoulli polynomials, the function $g$ is not necessarily equal Seki-Bernoulli polynomial on all $m \in \mathbb{N}$, but, by Lemma 4.7.7, $\partial g$ equals some polynomial. Also, using $g$ is uniquely determined by $\partial g$, the function $g$ equals some polynomial. We expand $g$ as a linear combination of Seki-Bernoulli polynomials.

Definition 4.11.1. Given integers $l_{1}, \ldots, l_{n}$, we define the structure constants $C_{i}^{\boldsymbol{l}}$ by

$$
g\left(\mathcal{F}_{l_{1}}, \ldots, \mathcal{F}_{l_{n}}\right)=\sum_{i=0}^{|l|-1} C_{i}^{l} \mathcal{F}_{|l|-i}
$$

Observe that $C_{i}^{l}=0$ for odd $i$, as $\partial g$ is even or odd. Corollary 4.7.6 is the statement

$$
C_{i}^{(1, \ldots, 1)}= \begin{cases}1 & i=0 \\ 0 & \text { else. }\end{cases}
$$

More generally, by Theorem 4.7.4(ii) one has $C_{i}^{1, l}=C_{i}^{l}$, so that w.l.o.g. we can assume $l_{i}>1$. In this section, we give an explicit, but involved, formula for these coefficients in terms of Bernoulli numbers and binomial coefficients. In order to do so, for $l_{1}, l_{2} \geq 1$ and $i \in \mathbb{Z}_{\geq 0}$, we introduce the following numbers:

$$
\mathcal{B}_{i}^{l_{1}, l_{2}}:= \begin{cases}\frac{\left(l_{1}-1\right) !\left(l_{2}-1\right) !}{\left(l_{1}+l_{2}-1\right) !} & i=0 \\
\zeta(1-i)\left((-1)^{l_{2}}\left(\begin{array}{c}
l_{1}-1 \\
i-l_{2}
\end{array}\right)+(-1)^{l_{1}}\left(\begin{array}{c}
l_{2}-1 \\
i-l_{1}
\end{array}\right)\right) & i>0,\end{cases}
$$

which by [AIK14, Proposition A.10] satisfy

$$
\sum_{i=0}^{l_{1}+l_{2}-2} \mathcal{B}_{i}^{l_{1}, l_{2}} \frac{B_{l_{1}+l_{2}-i}}{l_{1}+l_{2}-i}=(-1)^{l_{1} l_{2}} \frac{B_{l_{1}+l_{2}}-B_{l_{1}} B_{l_{2}}}{l_{1} l_{2}} .
$$

Note that $\zeta(1-i)=(-1)^{i+1} \frac{B_{i}}{i}$ for $i \geq 1$. The following polynomials can be expressed in terms of these coefficients:

Lemma 4.11.2. For all $l_{1}, l_{2}, \ldots, l_{r} \geq 2$ one has the following identities:

(i) $\mathcal{F}_{l_{1}}(x)=\sum_{i=0}^{\infty} \mathcal{B}_{i}^{l_{1}, 1} x^{l_{1}-i}$;

(ii) $\left(\partial \mathcal{F}_{l_{1}} * \partial \mathcal{F}_{l_{2}}\right)(x)=\sum_{i=0}^{\infty} \mathcal{B}_{i}^{l_{1}, l_{2}} x^{l_{1}+l_{2}-i-1}$; 
(iii) $\partial\left(\mathcal{F}_{l_{1}} \cdots \mathcal{F}_{l_{r}}\right)(x)=2 \sum_{|\boldsymbol{i}| \equiv 1(2)} \mathcal{B}_{i_{1}}^{l_{1}, 1} \cdots \mathcal{B}_{i_{r}}^{l_{r}, 1} x^{|\boldsymbol{l}|-|\boldsymbol{i}|}$.

Proof. The first two equations, of which the former is the well-known expansion of the Seki-Bernoulli polynomials, follow by considering the corresponding generating series. In order to prove (ii), we let $n \in \mathbb{N}$ and consider

$$
\begin{aligned}
\mathcal{G}(n) & :=\sum_{l_{1}, l_{2}=1}^{\infty}\left(\partial \mathcal{F}_{l_{1}} * \partial \mathcal{F}_{l_{2}}\right)(n) \frac{z_{1}^{l_{1}-1}}{\left(l_{1}-1\right) !} \frac{z_{2}^{l_{2}-1}}{\left(l_{2}-1\right) !} \\
& =\sum_{m_{1}+m_{2}=n} e^{m_{1} z_{1}+m_{2} z_{2}} \\
& =\frac{e^{n z_{1}}}{e^{z_{1}-z_{2}}-1}+\frac{e^{n z_{2}}}{e^{z_{2}-z_{1}}-1} .
\end{aligned}
$$

As the generating series of the Bernoulli numbers $\sum_{j=0}^{\infty} B_{j} \frac{z^{j}}{j !}=z\left(e^{z}-1\right)^{-1}$ implies that

$$
\frac{1}{e^{z_{1}-z_{2}}-1}=\frac{1}{z_{1}-z_{2}}+\sum_{j=1}^{\infty} \sum_{i=0}^{j-1} \frac{B_{j}}{j}(-1)^{i} \frac{z_{1}^{j-i-1} z_{2}^{i}}{(j-1-i) ! i !},
$$

we find

$$
\mathcal{G}(n)=\sum_{l_{1}, l_{2}=1}^{\infty} \sum_{i=0}^{\infty}(-1)^{i} \mathcal{B}_{i}^{l_{1}, l_{2}} \frac{z_{1}^{l_{1}-1}}{\left(l_{1}-1\right) !} \frac{z_{2}^{l_{2}-1}}{\left(l_{2}-1\right) !} n^{l_{1}+l_{2}-i-1} .
$$

Since $\mathcal{B}_{i}^{l_{1}, l_{2}}$ vanishes for odd $i$ if $l_{1}, l_{2}>1$, this proves the second equation. The third equation follows from the first by noting that

$$
\partial\left(\mathcal{F}_{l_{1}} \cdots \mathcal{F}_{l_{r}}\right)(x)=\left(\mathcal{F}_{l_{1}} \cdots \mathcal{F}_{l_{r}}\right)(x)-(-1)^{|l|}\left(\mathcal{F}_{l_{1}} \cdots \mathcal{F}_{l_{r}}\right)(-x) .
$$

Using these identities, one obtains

$$
C_{i}^{l}=\mathcal{B}_{i}^{1,1}=\delta_{i, 0}, \quad C_{i}^{l_{1}, l_{2}}=\mathcal{B}_{i}^{l_{1}, 1}+\mathcal{B}_{i}^{l_{2}, 1}-\mathcal{B}_{i}^{l_{1}, l_{2}} .
$$

These easy expressions for small $n$ are misleading, as $6 C_{i}^{l_{1}, l_{2}, l_{3}}$ equals

$$
\begin{aligned}
\frac{1}{4} \delta_{i, 2}+3 \sum_{\substack{i_{1}, i_{2} \equiv 0(2) \\
i_{1}+i_{2}=i}} \mathcal{B}_{i_{1}}^{l_{1}, 1} \mathcal{B}_{i_{2}}^{l_{2}, 1}-\sum_{\substack{i_{1} \equiv 1(2), j_{1} \\
i_{1}+j_{1}=i}} \mathcal{B}_{i_{1}}^{l_{1}, 1} \mathcal{B}_{j_{1}}^{l_{1}, l_{2}+l_{3}-i_{1}}+ \\
+2 \sum_{j_{1}+j_{2}=i} \mathcal{B}_{j_{1}}^{l_{1}, l_{2}} \mathcal{B}_{j_{2}}^{l_{1}+l_{2}-j_{1}, l_{3}}
\end{aligned}
$$


up to full symmetrisation, i.e., summing over all $\sigma \in S_{3}$ with $l_{i}$ replaced by $l_{\sigma(i)}$. In general, given $\alpha \in \Pi(n)$, write $\alpha=\left\{A_{1}, \ldots, A_{r}\right\}$ and denote $A^{j}=\cup_{i=1}^{j} A_{j}$. Also, for a vector $\boldsymbol{k}$ and a set $B$ we let $k_{B}=\sum_{b \in B} k_{b}$. Then, the above observations allows us to write down the following formula, which is very amenable to computer calculation:

Proposition 4.11.3. Let $l_{1}, \ldots, l_{n}>1$. Then, $C_{i}^{l}$ is given by

$$
\sum_{\alpha \in \Pi(n)} 2^{r} \mu(\alpha, \mathbf{1}) \sum_{\substack{i_{1}, \ldots, i_{n} \\\left|\boldsymbol{i}_{A}\right| \equiv 1(2)}}\left(\prod_{k=1}^{n} \mathcal{B}_{i_{k}}^{l_{k}, 1}\right)\left(\sum_{\substack{j_{1}, \ldots, j_{\ell(\alpha)-1} \\|\boldsymbol{i}|+|\boldsymbol{j}|=i+r}} \prod_{s=1}^{r-1} \mathcal{B}_{j_{s}}^{l_{A^{s}}-j_{s-1}, l_{A_{s+1}}-i_{A_{s+1}}+1}\right) .
$$

Here, $j_{0}:=l_{A_{0}}-i_{A_{0}}$.

Note that the latter formula is written in an asymmetric way, but (by associativity of the convolution product) is symmetric in the $l_{i}$.

\section{From the pointwise product to the induced product}

Suppose an element of $\mathcal{T}$ is given, written in the basis with respect to the pointwise product. How do we determine its (possibly mixed) weight and its representation in terms of the basis with respect to the $\odot$ product? A first answer is given by applying Möbius inversion to Equation (4.16), as given by Equation (4.36), i.e.,

$$
T_{\boldsymbol{k}, \boldsymbol{l}}=\sum_{\alpha \in \Pi(n)} \bigodot_{A \in \alpha}\left(T_{k_{A_{1}}, l_{A_{1}}}\left|T_{k_{A_{2}}, l_{A_{2}}}\right| \ldots\right) .
$$

However, as every factor $T_{k_{A_{1}}, l_{A_{1}}}\left|T_{k_{A_{2}}, l_{A_{2}}}\right| \ldots$ in the above equation is a linear combination of generators of different weights, it is useful to have a recursive version of this result. For this, we write $\frac{\partial}{\partial T_{k, l}}$ for the derivative of $f \in \mathcal{T}$ in the former basis (with respect to the pointwise product) and $\frac{\partial}{\partial T_{k, l}}$ for $\prod_{i} \frac{\partial}{\partial T_{k_{i}, l_{i}}}$.

Proposition 4.11.4. Let $k, l \geq 1$. There exist differential operators $\mathfrak{s}_{i, j}$ for all $i, j \in$ $\mathbb{Z}$ such that $\mathfrak{s}_{i, j}=0$ if $j<0$ and for all $f \in \mathcal{T}$ one has

$$
T_{k, l} f=\sum_{i \geq 0} \sum_{j \geq-l+1} T_{k+i, l+j} \odot \mathfrak{s}_{i, j}(f) .
$$

Explicitly,

$$
\mathfrak{s}_{i, j}=\sum_{|\boldsymbol{a}|=i} \mathfrak{t}_{\boldsymbol{a}, j}, \quad \mathfrak{t}_{\boldsymbol{a}, j}=\sum_{\boldsymbol{b}} C_{|\boldsymbol{b}|-j}^{l, \boldsymbol{b}} \frac{\partial}{\partial T_{\boldsymbol{a}, \boldsymbol{b}}},
$$


where $\boldsymbol{a}$ and $\boldsymbol{b}$ are vectors of integers of the same length and with $|\boldsymbol{a}|=i$, the structure constants $C_{|\boldsymbol{b}|-j}^{l, \boldsymbol{b}}$ are as in Proposition 4.11 .3 and l, $\boldsymbol{b}$ denotes the vector $\left(l, b_{1}, b_{2}, \ldots\right)$.

Proof. By linearity, it suffices to prove the statement for monomials $T_{k, l}$. Hence, assume $f=T_{k, l}$. Applying (4.43), extracting the factor containing $T_{k, l}$ and applying (4.43) again, yields

$$
\begin{aligned}
T_{k, l} f & =\sum_{A \subset[n]}\left(T_{k, l}\left|T_{k_{A_{1}}, l_{A_{1}}}\right| T_{k_{A_{2}}, l_{A_{2}}} \mid \ldots\right) \odot T_{\boldsymbol{k}_{[n] \backslash A}, l_{[n] \backslash A}} \\
& =\sum_{\boldsymbol{a}, \boldsymbol{b}}\left(T_{k, l}\left|T_{a_{1}, b_{1}}\right| T_{a_{2}, b_{2}} \mid \ldots\right) \odot \frac{\partial}{\partial T_{\boldsymbol{a}, \boldsymbol{b}}} f .
\end{aligned}
$$

By Definition 4.11.1 this equals

$$
T_{k, l} f=\sum_{\boldsymbol{a}, \boldsymbol{b}} \sum_{j \in \mathbb{Z}} C_{j}^{l, \boldsymbol{b}} T_{|\boldsymbol{a}|+k,|\boldsymbol{b}|+l-j} \odot \frac{\partial}{\partial T_{\boldsymbol{a}, \boldsymbol{b}}} f
$$

Replacing $j$ by $-j+|\boldsymbol{b}|$ and writing $i=|\boldsymbol{a}|$, one obtains

$$
T_{k, l} f=\sum_{i \geq 0} \sum_{j \in \mathbb{Z}} T_{k+i, l+j} \odot \sum_{|\boldsymbol{a}|=i} \sum_{\boldsymbol{b}} C_{|\boldsymbol{b}|-j}^{l, \boldsymbol{b}} \frac{\partial}{\partial T_{\boldsymbol{a}, \boldsymbol{b}}} f,
$$

as desired.

Corollary 4.11.5. For all $k, l \geq 1$ and $f \in \mathcal{T}$ one has

$$
\left\langle T_{k, l} f\right\rangle_{q}=\sum_{a \geq 0} \sum_{b \geq 2} D^{a} G_{b}\left\langle\mathfrak{T}_{k, l}^{a, b} f\right\rangle_{q}
$$

where $\mathfrak{T}_{k, l}^{a, b}=\mathfrak{s}_{a-l+1, a+b-k-1}+\mathfrak{s}_{a+b-l, a-k}$.

Proof. Distinguishing two cases in the previous result yields

$$
\begin{aligned}
\left\langle T_{k, l} f\right\rangle_{q}= & \sum_{j<k+i-l} D^{l+j-1} G_{k+i-l-j+2} \odot \mathfrak{s}_{i, j}(f)+ \\
& +\sum_{i \geq 0} \sum_{j \geq k+i-l} D^{k+i} G_{l+j-k-i} \odot \mathfrak{s}_{i, j}(f) \\
= & \sum_{a \geq 0} \sum_{b \geq 2} D^{a} G_{b}\left\langle\left(\mathfrak{s}_{a+b-k-1, a-l+1}+\mathfrak{s}_{a-k, a+b-l}\right)(f)\right\rangle_{q} .
\end{aligned}
$$




\subsection{Border strip moments and moments of other partition invariants}

We apply our results to interesting functions on partitions. First, we study the Möller transformation (defined in [Zag16, Eqn (45)] and recalled below) of elements in $\mathcal{T}$. In order to do so, let us first recall four equivalent definition of the hook-length moments, among which as the Möller transformations of the elements in $\mathcal{S}$.

\section{Hook-length moments}

Denote $z_{\nu}=\frac{n !}{\left|C_{\nu}\right|}$ with $\left|C_{\nu}\right|$ the size of the conjugacy class corresponding to $\nu$. Recall that

$$
z_{\nu}=\prod_{m=1}^{\infty} m^{r_{m}(\nu)} r_{m}(\nu) !
$$

Definition 4.12.1. Given $f \in \mathbb{Q}^{\mathscr{P}}$, the Möller transform of $f$ at a partition $\lambda \in \mathscr{P}(n)$ is given by

$$
\mathcal{M}(f)(\lambda)=\sum_{\nu \vdash n} z_{\nu}^{-1} \chi^{\lambda}(\nu)^{2} f(\nu),
$$

where the sum $\nu \vdash n$ is over all partitions of size $n$ and $\chi^{\lambda}(\rho)$ denotes the the character of the representation corresponding to the partition $\lambda$ evaluated at the conjugacy class corresponding to $\rho$.

Then $\langle\mathcal{M}(f)\rangle_{q}$ is a quasimodular form if and only if $\langle f\rangle_{q}$ is a quasimodular form (which follows directly by the column orthogonality relations for the symmetric group). Now, an alternative expression for $H_{k}$ is

$$
H_{k}=\mathcal{M}\left(S_{k}\right) .
$$

A second expression for $H_{k}$ makes use of certain constructions related to the Young diagram. Given partitions $\lambda, \nu$ with $\nu_{i} \leq \lambda_{i}$ for all $i$, we define the skew Young diagram $\lambda / \nu$ by removing the cells of $Y_{\nu}$ from $Y_{\lambda}$. Denote by $|\lambda / \nu|=|\lambda|-|\nu|$ the number of cells of this diagram. We call $\lambda / \nu$ a border strip of $\lambda$ if it is connected (through edges, not only through vertices) and contains no $2 \times 2$-block. If $\gamma=\lambda / \nu$ we write $\lambda \backslash \gamma$ for $\nu$. The height of a border strip $\gamma$ is defined to be one less than the number of columns and denoted by ht $(\gamma)$. Given $\boldsymbol{m} \in \mathbb{N}^{s}$, we let a border strip tableau $\gamma$ of type $\boldsymbol{m}$ be a sequence $\gamma_{1}, \ldots, \gamma_{s}$ such that $\gamma_{i}$ is a border strip of $\lambda$ 入 $\gamma_{1} \backslash \cdots \backslash \gamma_{i-1}$ and $\left|\gamma_{i}\right|=m_{i}$. Write $Y_{\gamma}$ for the skew Young diagram consisting of all boxes of all the $\gamma_{i}$ and write $\operatorname{ht}(\gamma)=\operatorname{ht}\left(\gamma_{1}\right)+\ldots+\operatorname{ht}\left(\gamma_{s}\right)$. Denote by $\operatorname{BST}(\lambda, \boldsymbol{m})$ and $\operatorname{BST}(\lambda / \nu, \boldsymbol{m})$ the set of all border strip tableau of type $\boldsymbol{m}$ within $\lambda$ and $\lambda / \nu$, respectively. 

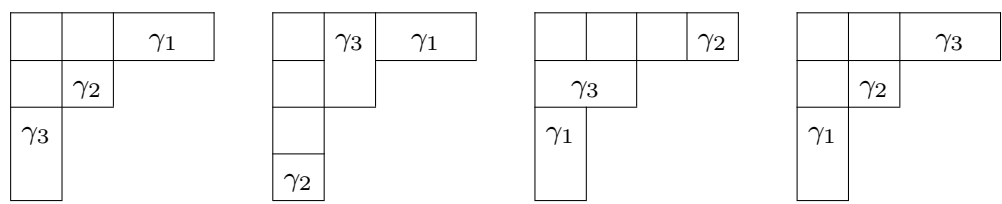

Figure 4.1: The Young diagrams corresponding to the border strip tableaux of type $(2,1,2)$ within $\lambda=(4,2,1,1)$.

The Murnaghan-Nakayama rule (recursively) expresses the characters of the symmetric groups in terms the heights of border strip tableau. Namely, if $\rho^{\prime} \subseteq \rho$ (both $\rho^{\prime}$ and $\rho$ considered as multisets)

$$
\chi^{\lambda}(\rho)=\sum_{\gamma \in \operatorname{BST}\left(\lambda, \rho^{\prime}\right)}(-1)^{\mathrm{ht}(\gamma)} \chi^{\lambda \backslash \gamma}\left(\rho-\rho^{\prime}\right),
$$

where $\rho-\rho^{\prime}$ denotes the difference of (multi)sets. Of particular interest are the cases $\rho^{\prime}=\rho$ and $\rho^{\prime}=\left(\rho_{1}\right)$, yielding a direct or recursive combinatorial formula for $\chi^{\lambda}(\rho)$, respectively:

$$
\chi^{\lambda}(\rho)=\sum_{\gamma \in \operatorname{BST}(\lambda, \rho)}(-1)^{\mathrm{ht}(\gamma)} \quad \text { and } \quad \chi^{\lambda}(\rho)=\sum_{|\gamma|=\rho_{1}}(-1)^{\mathrm{ht}(\gamma)} \chi^{\lambda \backslash \gamma}\left(\rho_{2}, \rho_{3}, \ldots\right),
$$

where the latter sum is over all borders strips $\gamma$ of $\lambda$ of length $\rho_{1}$. The skew charac$\operatorname{ter} \chi^{\lambda / \nu}\left(\rho^{\prime}\right)$ is defined by $\left(|\lambda / \nu|=\left|\rho^{\prime}\right|\right)$

$$
\chi^{\lambda / \nu}\left(\rho^{\prime}\right)=\sum_{\gamma \in \operatorname{BST}\left(\lambda / \nu, \rho^{\prime}\right)}(-1)^{\mathrm{ht}(\gamma)},
$$

so that

$$
\chi^{\lambda}(\rho)=\sum_{|\nu|=\left|\rho^{\prime}\right|} \chi^{\lambda / \nu}\left(\rho^{\prime}\right) \chi^{\nu}\left(\rho-\rho^{\prime}\right) .
$$

Now, we have the following definitions of the hook-length moments.

Definition 4.12.2. The hook-length moments $H_{k}(k \geq 2$ even) are defined by either of the following equivalent definitions [CMZ18, Section 13]:

(i) $H_{k}(\lambda)=-\frac{B_{k}}{2 k}+\sum_{\xi \in Y_{\lambda}} h(\xi)^{k-2}$;

(ii) $H_{k}(\lambda)=-\frac{B_{k}}{2 k}+\sum_{m=1}^{\infty}|\operatorname{BST}(\lambda, m)| m^{k-2}$; 
(iii) $H_{k}=\frac{1}{2}(k-1) ! \sum_{i=0}^{k}(-1)^{i} Q_{i} Q_{k-i}$;

(iv) $H_{k}=\mathcal{M}\left(S_{k}\right)$.

\section{The Möller transform of double moment functions}

We express elements of $\mathcal{T}$ in terms of functions $U_{\boldsymbol{k}, l}$ for which the induced product and Möller transformation are easy to compute. However, these function do not admit the property that the $q$-bracket is quasimodular if $k_{i}+l_{i}$ is even for all $i$ : each $U_{\boldsymbol{k}, \boldsymbol{l}}$ lies in the space generated by all the $T_{\boldsymbol{k}, l}$ (possibly with $k_{i}+l_{i}$ odd).

Given a vector of integers $l$, let

$$
\mathbb{N}(\boldsymbol{l})=\left\{(\underbrace{m_{1}, \ldots, m_{1}}_{l_{1}}, \underbrace{m_{2}, \ldots, m_{2}}_{l_{2}}, \ldots) \mid m_{i} \geq 1\right\}
$$

the set of tuples of $n:=|\boldsymbol{l}|$ positive integers, where the first $l_{1}$, the second $l_{2}$, etc. integers agree. For $\boldsymbol{k} \in \mathbb{Z}_{\geq 0}^{n}$, define

$$
U_{\boldsymbol{k}, \boldsymbol{l}}=\sum_{\boldsymbol{m} \in \mathbb{N}(\boldsymbol{l})} \boldsymbol{m}^{k} \prod_{a=1}^{\infty}\left(\begin{array}{c}
r_{a}(\lambda) \\
r_{a}(\boldsymbol{m})
\end{array}\right) .
$$

Observe that this product converges since $r_{a}(\boldsymbol{m})=0$ for all but finitely many values of $a$. Let $\mathcal{U}$ be the algebra generated by the $U_{k, l}$. Generalise the hook-length moments in Definition 4.12.2(ii) by the following notion:

Definition 4.12.3. The border strip moments are given by

$$
X_{\boldsymbol{k}, \boldsymbol{l}}(\lambda)=\sum_{\boldsymbol{m} \in \mathbb{N}(\boldsymbol{l})} \sum_{\gamma \in \operatorname{BST}(\lambda, \boldsymbol{m})} \frac{\chi^{\gamma}(\boldsymbol{m})^{2}}{z_{\boldsymbol{m}}} \boldsymbol{m}^{\boldsymbol{k}} .
$$

Let $\mathcal{X}$ be the vector space spanned by all the $X_{\boldsymbol{k}, l}$. Define a filtration on $\mathcal{X}$ by assigning to $X_{k, l}$ degree $|\boldsymbol{k}|+|\boldsymbol{l}|$.

Remark 4.12.4. Observe that for $n=1$ and $l=1$, the sum restricts to a sum over all border strips $\gamma$ of $\lambda$ and for such a border strip $\gamma$ the factor $\frac{\chi^{\gamma}(\boldsymbol{m})^{2}}{z_{m}}$ equals 1 and $z_{m}$ equals $m$. As the set of hook-lengths is in bijection with the set of all border strip lengths, one has that $-\frac{B_{k}}{2 k}+X_{k, 1}=H_{k+1}$.

Denote by $\left\{\begin{array}{l}n \\ j\end{array}\right\}$ the Stirling numbers of the second kind (i.e., the number of elements in $\Pi(n)$ of length $j$ ). 
Proposition 4.12.5. For all $k \geq 0, l \geq 1, \boldsymbol{k}, \boldsymbol{k}^{\prime} \in \mathbb{Z}_{\geq 0}^{n}$, and integer vectors $\boldsymbol{l}, \boldsymbol{l}^{\prime}$ with $|\boldsymbol{l}|=\left|\boldsymbol{l}^{\prime}\right|=n$, one has

(i) $T_{k, l}=-\frac{B_{k+l}}{2(k+l)}\left(\delta_{l, 1}+\delta_{k, 0}\right)+\sum_{j=1}^{l}\left\{\begin{array}{l}l \\ j\end{array}\right\}(j-1) ! U_{k, j}$;

(ii) $U_{\boldsymbol{k}, \boldsymbol{l}} \odot U_{\boldsymbol{k}^{\prime}, \boldsymbol{l}^{\prime}}=U_{\boldsymbol{k} \cup \boldsymbol{k}^{\prime}, l \cup \boldsymbol{l}^{\prime}}$;

(iii) $\mathcal{M}\left(U_{k, l}\right)=X_{k, l}$.

Proof. For the first property, we use the known identity

$$
x^{l-1}=\sum_{j=1}^{l}\left\{\begin{array}{l}
l \\
j
\end{array}\right\}(j-1) !\left(\begin{array}{l}
x-1 \\
j-1
\end{array}\right) .
$$

As $\mathcal{F}_{l}(x)$ and $\left(\begin{array}{l}x \\ j\end{array}\right)$ are the unique polynomials with constant term equal to zero and such that $\partial \mathcal{F}_{l}(x)=x^{l-1}$ and $\partial\left(\begin{array}{l}x \\ j\end{array}\right)=\left(\begin{array}{c}x-1 \\ j-1\end{array}\right)$, respectively, we find

$$
\mathcal{F}_{l}(x)=\sum_{j=1}^{l}\left\{\begin{array}{l}
l \\
j
\end{array}\right\}(j-1) !\left(\begin{array}{l}
x \\
j
\end{array}\right),
$$

which yields property (i).

Next, we show that for all $i, j \geq 0$ one has

$$
\partial\left(\begin{array}{c}
x \\
i
\end{array}\right) *\left(\begin{array}{c}
x \\
j
\end{array}\right)=\left(\begin{array}{c}
x \\
i+j
\end{array}\right) .
$$

Both

$$
\left(\begin{array}{c}
x \\
i
\end{array}\right) *\left(\begin{array}{c}
x \\
j
\end{array}\right)=\sum_{m=0}^{x}\left(\begin{array}{c}
m \\
i
\end{array}\right)\left(\begin{array}{c}
x-m \\
j
\end{array}\right) \quad \text { and } \quad\left(\begin{array}{c}
x+1 \\
i+j+1
\end{array}\right)
$$

are polynomials of degree at most $i+j+1$ taking the value 0 for $x=0,1, \ldots, i+j-1$ and the value 1 for $x=i+j$; hence, they are equal. Therefore,

$$
\partial\left(\begin{array}{c}
x \\
i
\end{array}\right) *\left(\begin{array}{c}
x \\
j
\end{array}\right)=\partial\left(\begin{array}{c}
x+1 \\
i+j+1
\end{array}\right)=\left(\begin{array}{c}
x \\
i+j
\end{array}\right) .
$$

By Lemma 4.7.2 property (ii) follows.

Finally, we have that

$$
\mathcal{M}\left(U_{\boldsymbol{k}, \boldsymbol{l}}\right)(\lambda)=\sum_{\boldsymbol{m} \in \mathbb{N}(\boldsymbol{l})} \boldsymbol{m}^{k} \sum_{\nu \vdash n} z_{\nu}^{-1} \chi^{\lambda}(\nu)^{2} \prod_{a=1}^{\infty}\left(\begin{array}{c}
r_{a}(\nu) \\
r_{a}(\boldsymbol{m})
\end{array}\right) .
$$


Observe that given $\boldsymbol{m}$ and $\nu$ the term

$$
z_{\nu}^{-1} \chi^{\lambda}(\nu)^{2} \prod_{a=1}^{\infty}\left(\begin{array}{c}
r_{a}(\nu) \\
r_{a}(\boldsymbol{m})
\end{array}\right)
$$

vanishes unless $r_{a}(\nu) \geq r_{a}(\boldsymbol{m})$ for all positive $a$. Let $\nu^{\prime}$ be the partition obtained from $\nu$ by removing $r_{a}(\boldsymbol{m})$ parts of size $a$ from $\nu$ for all positive $a$. Denote by $n^{\prime}=$ $n-|\boldsymbol{m}|$ the size of $\nu^{\prime}$. By the Murnaghan-Nakayama rule one has

$$
\chi^{\lambda}(\nu)=\sum_{\xi \in \operatorname{BS}(\lambda, \boldsymbol{m})} \chi^{\xi}(\boldsymbol{m}) \chi^{\lambda \backslash \xi}\left(\nu^{\prime}\right) .
$$

One has

$$
z_{\nu}^{-1} \prod_{a=1}^{\infty}\left(\begin{array}{c}
r_{a}(\nu) \\
r_{a}(\boldsymbol{m})
\end{array}\right)=\prod_{a=1}^{\infty} \frac{1}{a^{r_{a}(\nu)} r_{a}(\boldsymbol{m}) !\left(r_{a}(\nu)-r_{a}(\boldsymbol{m})\right) !}=\frac{1}{z_{\nu^{\prime}} z_{\boldsymbol{m}}} .
$$

Hence, (4.45) equals

$$
\sum_{\xi \in \operatorname{BS}(\lambda, \boldsymbol{m})} \sum_{\rho \in \operatorname{BS}(\lambda, \boldsymbol{m})} \frac{\chi^{\xi}(\boldsymbol{m}) \chi^{\rho}(\boldsymbol{m})}{z_{\boldsymbol{m}}} \sum_{\nu^{\prime} \vdash n^{\prime}} z_{\nu^{\prime}} \chi^{\lambda \backslash \xi}\left(\nu^{\prime}\right) \chi^{\lambda \backslash \rho}\left(\nu^{\prime}\right) .
$$

The orthogonality relation for the symmetric group is the statement

$$
\sum_{\nu^{\prime} \vdash n^{\prime}} z_{\nu^{\prime}} \chi^{\lambda \backslash \xi}\left(\nu^{\prime}\right) \chi^{\lambda \backslash \rho}\left(\nu^{\prime}\right)=\delta_{\lambda \backslash \xi, \lambda \backslash \rho} .
$$

Hence, we obtain the desired result.

The $q$-bracket of an element in $\mathcal{X}$ is not necesarily a quasimodular form. However, it always lies in the following space of $q$-analogues of zeta values, see [GKZ06].

Definition 4.12.6. Let $\mathcal{C}_{\leq \ell}$ be the $\mathbb{Q}$-vector space consisting of all polynomials in the combinatorial Eisenstein series

$$
G_{k}(\tau)=-\frac{B_{k}}{2 k}+\sum_{r=1}^{\infty} \sum_{m=1}^{\infty} m^{k-1} q^{m r}, \quad(k \geq 1, \text { not necesarily even })
$$

and their derivatives of weight $\leq \ell$, where to $D^{r} G_{k}$ we assign the weight $k+2 r$.

Now, Proposition 4.12.5 implies the following result:

Theorem 4.12.7. For all $f \in \mathcal{X}_{\leq k}$, one has $\langle f\rangle_{q} \in \mathcal{C}_{\leq k}$. 
Proof. By Proposition 4.12.5, $f$ equals the Möller transform of some polynomial in the $T_{k, l}$ with respect to the product $\odot$. Here, however, it may happen that $k+l$ is odd. Mutatis mutandis in either of three approaches in $\S \S 4.3$, we find that the $q$-bracket of $T_{k, l}$ lies in $\mathcal{C}_{k+l}$, which proves the result.

Theorem 4.12.8. For all weights $k$ one has $\mathcal{M}\left(\mathcal{T}_{k}\right) \subset \mathcal{X}_{\leq k}$. More precisely,

$$
\frac{\mathcal{M}\left(T_{k_{1}, l_{1}} \odot \cdots \odot T_{k_{n}, l_{n}}\right)}{\left(l_{1}-1\right) ! \cdots\left(l_{n}-1\right) !}=X_{\boldsymbol{k}, \boldsymbol{l}}+\text { elements in } \mathcal{X} \text { of lower degree } .
$$

Proof. Observe that Proposition 4.12.5 implies that $\mathcal{M}\left(\mathcal{T}_{k}\right) \subset \mathcal{X}_{\leq k}$. Equation (4.46) follows from this proposition after noting that the Möller transformation of $T_{k, l}-$ $(l-1) ! U_{k, l}$ has degree strictly smaller than $k+l$.

Example 4.12.9. The following two equations provide examples of linear combinations of elements of $\mathcal{X}$ with a quasimodular $q$-bracket whenever $k+l$ and $k_{i}$ are even integers.

$$
\begin{gathered}
\mathcal{M}\left(T_{k, l}\right)=-\frac{B_{k+l}}{2(k+l)}\left(\delta_{k, 1}+\delta_{l, 0}\right)+\sum_{j=1}^{l}\left\{\begin{array}{l}
l \\
j
\end{array}\right\}(j-1) ! X_{k, j}, \\
\mathcal{M}\left(S_{k_{1}} \odot S_{k_{2}} \odot \cdots \odot S_{k_{n}}\right)=\sum_{A \subset[n]}\left(\prod_{i \notin A} \frac{B_{k_{i}}}{2 k_{i}}\right) X_{k_{A},(1,1, \ldots, 1)} .
\end{gathered}
$$

See Appendix A.3 for a table of elements in $\mathcal{X}$ with quasimodular $q$-bracket and of small degree.

Remark 4.12.10. In many examples the $X_{k, l}$ are not shifted symmetric functions or generated by shifted symmetric functions under the induced product. For example, $\mathcal{M}\left(T_{0,2}\right) \neq \mathcal{M}\left(S_{2}\right)$ and besides $Q_{2}=\mathcal{M}\left(S_{2}\right)=S_{2}$ there are no other nontrivial functions generated by $\Lambda^{*}$ under the pointwise product. It remains an open question whether the elements of $\mathcal{X}$ are in some sense related to shifted symmetric functions.

\section{Moments of other partition invariants}

So far we provided many examples of functions on partitions in $\Lambda^{*}$ and $\mathcal{T}$ related to the representation theory of the symmetric group. Now, we see that many purely combinatorial notions lead to different bases for $\mathcal{S}$. We compare these bases to corresponding bases of $\Lambda^{*}$. Most of these bases take the following form. Suppose an 
index set $I$ and a sequence $\left\{s_{i}\right\}_{i \in I}^{\infty}$ of elements of $\mathbb{Q}^{\mathscr{P}}$ are given. Then, we define the $k$ th moment of $s$ by (whenever this sum converges)

$$
M_{k}(s)(\lambda)=\sum_{i \in I}\left(s_{i}(\lambda)^{k}-s_{i}(\emptyset)^{k}\right) .
$$

For example, let the functions $\boldsymbol{p}, \boldsymbol{q}$ for the index set $\mathbb{N}$ be given by

$$
p_{i}(\lambda)=\lambda_{i}, \quad q_{i}(\lambda)=\lambda_{i}-i .
$$

Then, by definition,

$$
S_{k}=S_{k}(\emptyset)+M_{k-1}(\boldsymbol{p}), \quad Q_{k}=Q_{k}(\emptyset)+M_{k-1}(\boldsymbol{q}) .
$$

Note that by definition $M_{k}(s)(\emptyset)=0$. As the functions below will not respect the weight grading anyway, we will not include a constant term.

The sequences $\boldsymbol{a}, \boldsymbol{c}, \boldsymbol{h}, \boldsymbol{x}$ of functions on partitions are of further interest. Define these sequence, indexed by $\xi=(i, j) \in \mathbb{Z}_{\geq 0}^{2}$, by 0 if $\xi \notin Y_{\lambda}$ and

$$
\begin{array}{ll}
a_{\xi}(\lambda): \text { arm length of } \xi & h_{\xi}(\lambda): \text { hook-length of } \xi \\
x_{\xi}(\lambda)=i & c_{\xi}(\lambda): \text { content of } \xi, \text { i.e., } i-j
\end{array}
$$

if $\xi \in Y_{\lambda}$ (for the content, see also (3.4)). For $\boldsymbol{h}$ and $\boldsymbol{c}$ it is known that the corresponding moment functions are shifted symmetric, for the latter see [KO94, Theorem 4]. The moment functions corresponding to $\boldsymbol{a}$ and $\boldsymbol{x}$ turn out to be equal and to be elements of $\mathcal{S}$.

\section{Theorem 4.12.11.}

$$
\mathcal{S}=\mathbb{Q}\left[M_{k}(\boldsymbol{a}) \mid k \geq 0 \text { even }\right]=\mathbb{Q}\left[M_{k}(\boldsymbol{x}) \mid k \geq 0 \text { even }\right] .
$$

Proof. As the Seki-Bernoulli polynomials $\mathcal{F}_{k}$ with $k$ odd form a basis for the space of all odd polynomials, the functions

$$
\sum_{i=1}^{\infty} \mathcal{F}_{k}\left(\lambda_{i}\right)=\sum_{i=1}^{\infty} \sum_{a=1}^{\lambda_{i}} a^{k-1}
$$

generate $\mathcal{S}$, which corresponds to the first equality in the statement. By interchanging the sums one obtains

$$
\sum_{i=1}^{\infty} \mathcal{F}_{k}\left(\lambda_{i}\right)=\sum_{a=1}^{\infty} a^{k-1} \sum_{m=a}^{\infty} r_{m}(\lambda)=\sum_{(i, j) \in Y_{\lambda}} i^{k-1} .
$$

Hence, the result is also true for $s=\boldsymbol{x}$. 
Remark 4.12.12. Note that for a given $i$ the number of $(i, j) \in Y_{\lambda}$ equals $\lambda_{i}^{\prime}$, where $\lambda^{\prime}$ is the conjugate partition of $\lambda$. Hence, (4.47) can be written as

$$
\sum_{i=1}^{\infty} i^{k-1} \lambda_{i}^{\prime}
$$

and consequently these functions for $k$ odd generate $\mathcal{S}$. Note that these functions are different from the $S_{k}\left(\lambda^{\prime}\right)$. In fact, the algebra generated by the $S_{k}\left(\lambda^{\prime}\right)$ is distinct from the algebra $\mathcal{S}$, in contrast to the algebra of shifted symmetric functions, for which $Q_{k}\left(\lambda^{\prime}\right)=(-1)^{k} Q_{k}(\lambda)$. 


\section{Part III}

\section{Quasi-Jacobi forms and applications}



CHAPTER 5

\section{Taylor coefficients of quasi-Jacobi forms}

\subsection{Introduction}

Let $\tau \in \mathfrak{h}$, the complex upper half plane and $z \in \mathbb{C}$. Write $e(x)=e^{2 \pi \mathrm{i} x}$ and $q=e(\tau)$. Consider the Jacobi theta function $\theta$, defined in (1.14), its derivatives and the Kronecker-Eisenstein series $E_{k}$, for $k \geq 2$ given by

$$
E_{k}(\tau, z)=\sum_{m, n \in \mathbb{Z}} \frac{1}{(z+m \tau+n)^{k}},
$$

and the Bloch-Okounkov n-point functions $F_{n}$ (see Definition 1.4.2). Do these functions have a common property? To answer this question, observe that the first two functions can be interpreted as generating functions of the Eisenstein series $e_{k}$. Namely,

$$
\begin{aligned}
& \Theta(\tau, z):=\frac{\theta(\tau, z)}{\theta^{\prime}(\tau, 0)}=2 \pi \mathrm{i} z \exp \left(-\sum_{m \geq 1} \frac{e_{2 m}(\tau)}{2 m} z^{2 m}\right) \\
& E_{k}(\tau, z)=\frac{1}{z^{k}}+(-1)^{k} \sum_{m \geq k / 2}\left(\begin{array}{c}
2 m-1 \\
k-1
\end{array}\right) e_{2 m}(\tau) z^{2 m-k} .
\end{aligned}
$$

In particular, the Taylor (or Laurent) coefficients around $z=0$ of these functions are polynomials in Eisenstein series, hence quasimodular forms-a property which is shared by the stronger notion of a Jacobi form. Just as $e_{2}$ transforms as a quasimodular form, so do $\theta^{(r)}$ and $F_{n}$ transform as quasi-Jacobi forms. Our answer to the question is that all these functions are quasi-Jacobi forms, introduced in Section 5.6.

In this chapter we determine conditions on the Taylor coefficients of a meromorphic function $\varphi: \mathfrak{h} \times \mathbb{C}^{n} \rightarrow \mathbb{C}$ for it to be a (quasi-)Jacobi form. In one direction, by the work of Eichler and Zagier [EZ85], it is known that for a Jacobi form the Taylor

This chapter, as well as the next two chapters, are based on [I21b]. 
coefficients around rational lattice points are quasimodular, or equivalently that certain linear combinations $\xi_{\ell}^{X}$ of derivatives of these Taylor coefficients are modular. For example, $\Theta$ is a weak Jacobi form, hence it satisfies

$$
(\Theta|X| \gamma)(\tau, z) \propto(\Theta \mid \gamma X)(\tau, z)
$$

for all $X=\left(\begin{array}{l}\lambda \\ \mu\end{array}\right) \in M_{2,1}(\mathbb{Q})$ and $\gamma \in \mathrm{SL}_{2}(\mathbb{Z})$, where the slash action is being recalled in Definition 5.2.2 and the implicit multiplicative constant is a root of unity depending on $X$ and $\gamma$. Hence, it follows that the Taylor coefficients of $\Theta$ around $z=\lambda \tau+\mu$ (after multiplying with a certain power of $q$ ) are quasimodular for some subgroup $\Gamma_{X}$ of $\mathrm{SL}_{2}(\mathbb{Z})$ consisting of $\gamma$ for which $\gamma X-X \in M_{1,2}(\mathbb{Z})$ (see (5.17)). In contrast, the weak quasi-Jacobi form $\Theta^{\prime}(\tau, z)=\frac{\theta^{\prime}(\tau, z)}{\theta^{\prime}(\tau, 0)}$ transforms as

$$
\begin{aligned}
\left(\Theta^{\prime}|X| \gamma\right)(\tau, z) \propto\left(\Theta^{\prime} \mid \gamma X\right)(\tau, z) & +\frac{c z}{c \tau+d}(\Theta \mid \gamma X)(\tau, z)+ \\
& +\lambda(\Theta \mid \gamma X)(\tau, z)-\frac{\lambda}{c \tau+d}(\Theta \mid \gamma X)(\tau, z) .
\end{aligned}
$$

Therefore, the Taylor coefficients of $\Theta^{\prime}+\lambda \Theta$ around $z=\lambda \tau+\mu$, rather than of $\Theta^{\prime}$, give rise to quasimodular forms for $\Gamma_{X}$. The main result on Taylor coefficients of quasi-Jacobi forms is given by Theorem 5.9.10 and summarised in Theorem E.

Part of the proof of this result consist in defining the 'Taylor coefficients' $g_{\ell}^{X}(\varphi)$ corresponding to a quasi-Jacobi form $\varphi$ (see Definition 5.9.2). These 'Taylor coefficients' are defined in terms of the actual Taylor coefficients of a family of functions $\varphi_{i, \boldsymbol{j}}$ (here $i \in \mathbb{Z}_{\geq 0}, \boldsymbol{j} \in\left(\mathbb{Z}_{\geq 0}\right)^{n}$ ) appearing in the transformation of $\varphi$. For example, the quasimodularity of the Taylor coefficients of $\Theta^{\prime}$ cannot be understood without referring to $\Theta$, which occurs in (5.3). The functions $\xi_{m}^{X}(\varphi)$ will be defined in terms of derivatives of these coefficients $g_{\ell}^{X}(\varphi)$.

In the statement of Theorem $E$ we assumed that all poles lie on specific hyperplanes. This is to assure that the multivariate Taylor expansion exists, which is for example the case for the $n$-point functions $F_{n}$. We introduce the notion of a (multivariate) strictly meromorphic Jacobi form and show that its zeros always lie on a finite union of rational hyperplanes. Strictly meromorphic Jacobi forms are meromorphic functions $\varphi: \mathfrak{h} \times \mathbb{C}^{n} \rightarrow \mathbb{C}$, transforming as Jacobi forms, and with the following assumption on the location of the poles: if $\boldsymbol{z} \in \mathbb{R}^{n} \tau+\mathbb{R}^{n}$ is a pole of $\varphi(\tau, \cdot)$ for some $\tau \in \mathfrak{h}$, it is a pole for almost all $\tau \in \mathfrak{h}$. Note that, in contrast to many authors, we do not assume that all poles lie at torsion points or at rational hyperplanes. It is by studying the orbits of the action of $\mathrm{SL}_{2}(\mathbb{Z})$ on $M_{2, n}(\mathbb{R})$ that we prove Theorem 5.4.2, which was summarised in Theorem $\mathrm{D}$. In case $n=1$, Theorem $\mathrm{D}$ implies that all poles of a strictly meromorphic Jacobi form are torsion points $z \in \mathbb{Q} \tau+\mathbb{Q}$, which is crucial in order to show that Fourier coefficients of meromorphic Jacobi forms are mock modular forms (see [Zwe02, DMZ14]). 


\section{Contents}

The definition of Jacobi forms in [EZ85] has been generalised in many ways. We provide a generalisation that incorporates several elliptic variables, characters, certain meromorphic functions, and quasi-Jacobi forms [Lib11, Boy15, OP19]. In particular, the normalised Jacobi theta function $\Theta$, the Kronecker-Eisenstein series $E_{k}$ and the Bloch-Okounkov $n$-point functions $F_{n}$ from the introduction will be examples throughout this chapter.

The results of this chapter will be applied in the subsequent chapters. The quasimodular transformation of the coefficients $g_{\ell}^{X}\left(F_{n}\right)$ implies the results on the BlochOkounkov theorem for congruence subgroups in Chapter 6. Moreover, in Chapter 7, we see that pulling back the functions $\xi_{m}^{X}\left(F_{n}\right)$ under the $q$-bracket leads to the definition of the projection $\pi$.

\section{Notation}

For $\tau \in \mathfrak{h}$, we write $L_{\tau}=\mathbb{Z} \tau+\mathbb{Z}$. As is customary, we often omit the dependence on the modular variable $\tau$ in any type of Jacobi form, e.g. we write $\Theta(z)$ for $\Theta(\tau, z)$. We write $\boldsymbol{a}=\left(a_{1}, \ldots, a_{n}\right)$ for a vector of elements and we write $|\boldsymbol{a}|$ for $a_{1}+\ldots+a_{n}$. For vectors $\boldsymbol{a}$ and $\boldsymbol{b}$, we write $\boldsymbol{a}^{\boldsymbol{b}}$ to denote $\prod_{i} a_{i}^{b_{i}}$. Also, given $X \in M_{2, n}(\mathbb{R})$, we often write $X=\left(\begin{array}{l}\boldsymbol{\lambda} \\ \boldsymbol{\mu}\end{array}\right)$ with $\boldsymbol{\lambda}, \boldsymbol{\mu} \in \mathbb{R}^{n}$ and for $\gamma \in \mathrm{SL}_{2}(\mathbb{Z})$, we write $\gamma=\left(\begin{array}{ll}a & b \\ c & d\end{array}\right)$, $\gamma X=\left(\begin{array}{c}\boldsymbol{\lambda}_{\boldsymbol{\mu}^{\gamma}}^{\gamma} \\ )\end{array}\right)$. In accordance with the action of $\mathrm{SL}_{2}(\mathbb{Z})$ on $\mathfrak{h}$, all actions of $\mathrm{SL}_{2}(\mathbb{Z})$ on other spaces are left actions.

\subsection{Strictly meromorphic Jacobi forms}

The definition of a strictly meromorphic Jacobi form $\varphi$ is subtle, excluding many meromorphic functions transforming as Jacobi forms. For example, the $j$-invariant, the reciprocal of the $e_{4}$ Eisenstein series or $\wp / \Delta$, where $\wp$ is the Weierstrass $\wp$ function and $\Delta$ the modular discriminant, are all non-examples of strictly meromorphic Jacobi forms. Namely, first of all, although a strictly meromorphic Jacobi form is meromorphic we want its Taylor coefficients in the elliptic variables to be holomorphic (rather than weakly holomorphic or meromorphic) quasimodular forms. But with this not everything has been said, the definition is even stricter: we require the poles of $\varphi$ to be "constant" in the modular variable $\tau$. Consider, for example, the Weierstrass $\wp$-function, which is an example of a strictly meromorphic Jacobi form. For every fixed $z \in \mathbb{C}$ (e.g., $z=\mathrm{i}$ ) the function $\wp(\tau, z)$ is a meromorphic function of $\tau$, as $\varphi(\tau, z)$ has a pole whenever $\tau$ is such that $z \in L_{\tau}$ (e.g., $\tau=z=\mathrm{i}$ ). However, for $\lambda, \mu \in \mathbb{R}$, the function $\varphi(\tau, \lambda \tau+\mu)$ is holomorphic, unless both $\lambda, \mu \in \mathbb{Z}$. 
That is, all poles of $\wp(\tau, z)$ are given by $z \in L_{\tau}$. To the contrary, we will see that $\wp^{-1}$ is not a strictly meromorphic Jacobi form, as the poles of $\wp^{-1}$ are not "constant" in $\tau$.

Before introducing strictly meromorphic Jacobi forms, we first recall the Jacobi group and its action on (meromorphic) functions.

Definition 5.2.1. For all $n \in \mathbb{N}$, the (discrete) Jacobi group $\Gamma_{n}^{J}$ of rank $n$ is defined as the semi-direct product $\Gamma_{n}^{J}=\mathrm{SL}_{2}(\mathbb{Z}) \ltimes M_{2, n}(\mathbb{Z})$ with respect to the left action of $\mathrm{SL}_{2}(\mathbb{Z})$ on $M_{2, n}(\mathbb{Z})$.

That is, an element of $\Gamma_{n}^{J}$ is a pair $(\gamma, X)$ with $\gamma \in \mathrm{SL}_{2}(\mathbb{Z}), X \in M_{2, n}(\mathbb{Z})$ and satisfies the group law $(\gamma, X)\left(\gamma^{\prime}, X^{\prime}\right)=\left(\gamma \gamma^{\prime}, \gamma^{\prime} X+X^{\prime}\right)$.

Let $M \in M_{n}(\mathbb{Q})$. We often make use of the associated quadratic form $Q_{M}$ and bilinear form $B_{M}$, given by

$$
Q_{M}(\boldsymbol{z})=\boldsymbol{z} M \boldsymbol{z}^{t}, \quad B_{M}\left(\boldsymbol{z}, \boldsymbol{z}^{\prime}\right)=\boldsymbol{z} M \boldsymbol{z}^{\prime t} .
$$

Definition 5.2.2. Given a meromorphic function $\varphi: \mathfrak{h} \times \mathbb{C}^{n} \rightarrow \mathbb{C}, k \in \mathbb{Z}$ and $M \in M_{n}(\mathbb{Q})$, for all $(\gamma, X) \in \Gamma_{n}^{J}$ write $\gamma=\left(\begin{array}{ll}a & b \\ c & d\end{array}\right)$ and $X=\left(\begin{array}{l}\boldsymbol{\lambda} \\ \boldsymbol{\mu}\end{array}\right)$ and let

(i) $\left(\left.\varphi\right|_{k, M} \gamma\right)(\tau, \boldsymbol{z}):=(c \tau+d)^{-k} \boldsymbol{e}\left(\frac{-c Q_{M}(\boldsymbol{z})}{c \tau+d}\right) \varphi\left(\frac{a \tau+b}{c \tau+d}, \frac{\boldsymbol{z}}{c \tau+d}\right)$;

(ii) $\left(\left.\varphi\right|_{M} X\right)(\tau, \boldsymbol{z}):=\boldsymbol{e}\left(Q_{M}(\boldsymbol{\lambda}+\boldsymbol{\mu})\right) \boldsymbol{e}\left(B_{M}(\boldsymbol{\lambda}, \boldsymbol{\lambda} \tau+2 \boldsymbol{z})\right) \varphi(\tau, \boldsymbol{z}+\boldsymbol{\lambda} \tau+\boldsymbol{\mu})$.

Moreover, we let $\left.\varphi\right|_{k, M}(\gamma, X):=\left.\left(\left.\varphi\right|_{k, M} \gamma\right)\right|_{M} X$, often omitting $k$ and $M$ from the notation.

Remark 5.2.3. Given $k \in \mathbb{Z}$ and $M \in M_{n}(\mathbb{Q})$, the slash operator defines an action of $\Gamma_{n}^{J}$ of weight $k$ and index $M$ on the space of all meromorphic functions $\varphi: \mathfrak{h} \times \mathbb{C}^{n} \rightarrow \mathbb{C}$.

Given $M \in M_{n}(\mathbb{Q})$, for $X, X^{\prime} \in M_{2, n}(\mathbb{Q})$, we let

$$
\begin{aligned}
& \rho(X)=\boldsymbol{e}\left(Q_{M}(\boldsymbol{\lambda})-B_{M}(\boldsymbol{\lambda}, \boldsymbol{\mu})+Q_{M}(\boldsymbol{\mu})\right), \\
& \zeta_{X, X^{\prime}}=\boldsymbol{e}\left(B_{M}\left(\boldsymbol{\lambda}^{\prime}, \boldsymbol{\mu}\right)-B_{M}\left(\boldsymbol{\lambda}, \boldsymbol{\mu}^{\prime}\right)\right),
\end{aligned}
$$

where $X=\left(\begin{array}{c}\lambda \\ \mu\end{array}\right)$ and $X^{\prime}=\left(\begin{array}{l}\lambda^{\prime} \\ \mu^{\prime}\end{array}\right)$. By extending the slash action to the real Jacobi group in Section 5.3, generalizing [EZ85, Theorem 1.4] to several variables and halfintegral index, we obtain the following functional equations.

Proposition 5.2.4. Given a meromorphic function $\varphi: \mathfrak{h} \times \mathbb{C}^{n} \rightarrow \mathbb{C}, k \in \mathbb{Z}$ and $M \in M_{n}(\mathbb{Q})$, for all $X, X^{\prime} \in M_{2, n}(\mathbb{R})$ and $\gamma \in \mathrm{SL}_{2}(\mathbb{Z})$ one has

$$
\rho(-X) \varphi|X| \gamma=\rho(-\gamma X) \varphi|\gamma| \gamma X
$$


and

$$
\begin{aligned}
\rho(-X) \rho\left(-X^{\prime}\right) \varphi|X| X^{\prime} & =\rho\left(-X^{\prime}\right) \rho(-X) \varphi\left|X^{\prime}\right| X \\
& =\rho\left(-X-X^{\prime}\right) \zeta_{X, X^{\prime}}\left(\varphi \mid X+X^{\prime}\right) .
\end{aligned}
$$

We recall from p. 4 that classical modular forms were defined as the invariants for a certain group action of the space $\mathrm{Hol}_{0}(\mathfrak{h})$ of holomorphic functions in $\mathfrak{h}$ satisfying a certain growth condition (that have at most polynomial growth near the boundary). With the remarks at the beginning of this section in mind, we now define strictly meromorphic Jacobi forms. A final subtlety in the definition below is coming from the fact that a meromorphic function in two or more variables always has points of indeterminacy (think of $x / y$ near the origin, whose limiting value depends on the angle of approach). Points of indeterminacy are not "generic", and we exclude these points when we say for instance, that a certain function $\varphi(\tau, \boldsymbol{z})$ has its poles precisely on certain hyperplanes for all generic $\tau \in \mathfrak{h}$.

Definition 5.2.5. Given $n \geq 0$, denote by $\operatorname{Mer}_{n}$ the space of meromorphic functions $\varphi: \mathfrak{h} \times \mathbb{C}^{n} \rightarrow \mathbb{C}$ such that for all $\boldsymbol{\lambda}, \boldsymbol{\mu} \in \mathbb{R}^{n}$ either $\boldsymbol{z}=\boldsymbol{\lambda} \tau+\boldsymbol{\mu}$ is a pole of $\varphi(\tau, \cdot)$ for all generic $\tau \in \mathfrak{h}$ or the function $\tau \mapsto \varphi(\tau, \boldsymbol{\lambda} \tau+\boldsymbol{\mu})$ belongs to $\operatorname{Hol}_{0}(\mathfrak{h})$. Moreover, given $M \in M_{n}(\mathbb{Q})$, denote by $\operatorname{Mer}_{n}^{M}$ the subspace of $\varphi \in \operatorname{Mer}_{n}$ for which $\left.\varphi\right|_{M} X \in \operatorname{Mer}_{0}$ for all $X \in M_{2, n}(\mathbb{Q})$. Let $\operatorname{Hol}_{n}$ and $\operatorname{Hol}_{n}^{M}$ be the subspace in $\operatorname{Mer}_{n}$ and $\operatorname{Mer}_{n}^{M}$, respectively, of holomorphic functions.

Definition 5.2.6. Let $k \in \mathbb{Z}$ and $M \in M_{n}(\mathbb{Q})$. A holomorphic, weak, or a strictly meromorphic Jacobi form of weight $k$, index $M$ and $\operatorname{rank} n$ for the Jacobi group $\Gamma_{n}^{J}$ is a function $\varphi$ in $\mathrm{Hol}_{n}^{M}, \mathrm{Hol}_{n}$ or $\mathrm{Mer}_{n}^{M}$, respectively, that is invariant under the action of $\Gamma_{n}^{J}$ of weight $k$ and index $M$ (i.e., $\left.\varphi\right|_{k, M} g=\varphi$ for all $g \in \Gamma_{n}^{J}$ ).

Remark 5.2.7. The space of Jacobi forms is trivial whenever $2 Q_{M}$ is a non-integral quadratic form, or, equivalently, when $m_{i j} \notin \frac{1}{4} \mathbb{Z}$ or $m_{i i} \notin \frac{1}{2} \mathbb{Z}$ for some $i \neq j$ and where $M=\left(m_{i j}\right)$. Namely, let $\varphi$ be a Jacobi form and $\tau \in \mathfrak{h}$ fixed. If $\varphi$ is non-zero of rank 1 it follows from the elliptic transformation law (Definition 5.2.2 (ii)) that the number of zeros minus the number of poles of $z \mapsto \varphi(\tau, z)$ in any fundamental domain for the action of $L_{\tau}$ on $\mathbb{C}$ is exactly $2 m$, where $M=(m)$ is the index of $\varphi$. For Jacobi forms of higher rank the integrability of $2 Q_{M}$ follows by noting that for fixed $\mu_{2}, \ldots, \mu_{n} \in \mathbb{C}$, functions of the form $z \mapsto \varphi\left(\tau, z, \mu_{2}, \mu_{3}, \ldots, \mu_{n}\right)$ and $z \mapsto \varphi\left(\tau, z, z, \mu_{3}, \ldots, \mu_{n}\right)$ still satisfy the elliptic transformation law.

Letting $M$ be the 0-dimensional matrix, a holomorphic Jacobi form (of rank 0) is just a modular form. More interestingly, the Kronecker-Eisenstein series (5.1) for $k \geq 3$ are examples of a strictly meromorphic Jacobi form of index (0) with an 
expansion given by

$$
E_{k}(\tau, z)=\frac{(-1)^{k}}{(k-1) !} D_{y}^{k-2}\left(\sum_{m \in \mathbb{Z}} \frac{y q^{m}}{\left(1-y q^{m}\right)^{2}}\right),
$$

where $y=\boldsymbol{e}(z)$ and $D_{y}=y \frac{\partial}{\partial y}$.

Closely related are the Weierstrass $\wp$-function and its derivative, that is, $\wp=$ $E_{2}-e_{2}$ and $\wp^{\prime}=-2 E_{3}$ (here $E_{2}$ is defined by (5.1) using the same summation order as for $e_{2}$ ). By [EZ85, Theorem 9.4] it follows that the space of weak Jacobi forms is given by

$$
J^{\text {weak }}=\mathbb{C}\left[A, B, C, e_{4}, e_{6}\right] /\left(C^{2}-4 A B^{3}+60 e_{4} A^{3} B+140 e_{6} A^{4}\right),
$$

where $A, B$ and $C$ are equal to $\Theta^{2}, \wp \Theta^{2}$ and $\wp^{\prime} \Theta^{4}$ respectively. Note that the relation $C^{2}=4 A B^{3}-60 e_{4} A^{3} B-140 e_{6} A^{4}$ is equivalent to the relation for the Weierstrass $\wp$-function. The following result, yielding an algebraic proof and extending [Lib11, Proposition 2.8 $]^{1}$, gives all strictly meromorphic Jacobi forms with only poles at the lattices points. The corresponding algebra is free, as the relation between $\wp$ and $\wp^{\prime}$ can be used to express $e_{6}$ in terms of the generators. As usual, we write $m$ (instead of the matrix $M=(m)$ ) for the index of a Jacobi form of rank 1 .

Proposition 5.2.8. Let $\varphi$ be a strictly meromorphic Jacobi form of rank 1 and index $m \in \frac{1}{2} \mathbb{Z}_{\geq 0}$ for which all poles $(\tau, z)$ satisfy $z \in L_{\tau}$. Then,

$$
\varphi \in \mathbb{C}\left[\wp, \wp^{\prime}, e_{4}\right] \Theta^{2 m} .
$$

Proof. First, we show that $f=\varphi \Theta^{-2 m}$ is a strictly meromorphic Jacobi form of index 0 with all poles $(\tau, z)$ satisfying $z \in L_{\tau}$. This follows from the claim that $\Theta^{-1}$ is a strictly meromorphic Jacobi form of index $-1 / 2$, weight 1 and with all the poles at the lattice points. In order to prove this claim, note that by the Jacobi triple product

$$
\Theta=\left(y^{1 / 2}-y^{-1 / 2}\right) \prod_{n \geq 1} \frac{\left(1-y q^{n}\right)\left(1-y^{-1} q^{n}\right)}{\left(1-q^{n}\right)^{2}} .
$$

It follows that $\Theta$ is a weak Jacobi form with all zeros at the lattice points $z \in L_{\tau}$. Moreover, for all $X \in M_{2, n}(\mathbb{Q})$ the function $\Theta \mid X$ does not vanish at infinity, from which the claim follows.

From now on, assume that $\varphi$ is of index 0, i.e., that the function $\varphi$ is an elliptic function. Write $\varphi=\varphi_{0}+\varphi_{1}$ with $\varphi_{0}$ and $\varphi_{1}$ the even and odd part of $\varphi$ respectively.

\footnotetext{
${ }^{1}$ The author states the result for Jacobi forms, which obviously is not meant to be holomorphic Jacobi forms. Although not stated explicitly, we assume he refers to strictly meromorphic Jacobi forms with only poles at lattice points.
} 
For $u, v \in \mathbb{R} \tau+\mathbb{R}$, write $u \sim v$ if $u \equiv v$ or $u \equiv-v \bmod L_{\tau}$. Then, for $i=0,1$ one has

$$
\varphi_{i}=\left(\wp^{\prime}\right)^{i} \prod_{j}\left(\wp-\wp\left(u_{j}(\tau)\right)\right)^{m_{j}}
$$

where $m_{j} \in \mathbb{Z}$ and $u_{j}(\tau)$ are representatives with respect to the above equivalence relation for the zeros and poles of $\varphi_{i}$ outside $L_{\tau}$. As both $\varphi_{0}$ and $\varphi_{1}$ do not admit poles outside the lattice, it follows that $m_{j}>0$. Hence, $\varphi$ is a polynomial in $\wp$ and $\wp^{\prime}$ where the coefficients are polynomials in the functions $\wp\left(u_{j}(\tau)\right)$. By the modular transformation every such coefficient is a modular form for $\mathrm{SL}_{2}(\mathbb{Z})$, hence an element of $\mathbb{C}\left[\wp, \wp^{\prime}, e_{4}\right]$.

Remark 5.2.9. Although the above result and many examples of strictly meromorphic Jacobi forms in the literature have only poles at $z \in L_{\tau}$, one easily constructs a strictly meromorphic Jacobi forms with poles at different places. Namely, if $\varphi$ is a Jacobi form with all poles at $z \in L_{\tau}$, then

$$
\varphi\left(\tau, z+\frac{1}{2}\right)+\varphi\left(\tau, z+\frac{1}{2} \tau\right)+\varphi\left(\tau, z+\frac{1}{2} \tau+\frac{1}{2}\right)
$$

is a Jacobi form for the same group, but now with the poles at $\frac{1}{2}, \frac{1}{2}+\frac{1}{2} \tau$ and $\frac{1}{2} \tau$ modulo the lattice $L_{\tau}$.

\subsection{The action of the Jacobi group}

In this section we prove Proposition 5.2.4, or, more precisely, we show that the real Jacobi group acts on all meromorphic functions $\varphi: \mathfrak{h} \times \mathbb{C}^{n} \rightarrow \mathbb{C}$.

In order to define the real Jacobi group, we let

$$
\begin{aligned}
\omega: M_{2, n}(\mathbb{R}) \times M_{2, n}(\mathbb{R}) & \rightarrow \operatorname{Sym}_{n}(\mathbb{R}) \\
(X, Y) & \mapsto-\frac{1}{2} X^{t} J Y+\frac{1}{2} Y^{t} J X
\end{aligned}
$$

and

$$
\begin{aligned}
\iota: M_{2, n}(\mathbb{R}) \times M_{2, n}(\mathbb{R}) & \rightarrow \operatorname{Skew}_{n}(\mathbb{R}) \\
(X, Y) & \mapsto X^{t} J Y,
\end{aligned}
$$

where $J=\left(\begin{array}{cc}0 & -1 \\ 1 & 0\end{array}\right)$ and $\mathrm{Sym}_{n}$ and $\mathrm{Skew}_{n}$ denote the space of symmetric and skewsymmetric $n \times n$-matrices, respectively. Then, we define $H_{n}$ to be the central extension of $M_{2, n}(\mathbb{R})$ by $\operatorname{Sym}_{n}(\mathbb{R})$ corresponding to the 2 -cocycle $\omega$, so that

$$
H_{n}=\left\{(X, \kappa): X \in M_{2, n}(\mathbb{R}), \kappa+\frac{1}{2} \iota(X, X) \in \operatorname{Sym}_{n}(\mathbb{R})\right\}
$$


with multiplication

$$
(X, \kappa)\left(X^{\prime}, \kappa^{\prime}\right)=\left(X+X^{\prime}, \kappa+\kappa^{\prime}-\iota\left(X, X^{\prime}\right)\right) .
$$

Remark 5.3.1. Observe that if $n=1$, this group $H_{1}$ is isomorphic to the Heisenberg group $H_{\mathbb{R}}$ with underlying set $\mathbb{R}^{2} \times \mathbb{R}$ and product given by

$$
(X, \kappa)\left(X^{\prime}, \kappa^{\prime}\right)=\left(X+X^{\prime}, \kappa+\kappa^{\prime}+\operatorname{det}\left(X, X^{\prime}\right)\right) .
$$

Definition 5.3.2. The real Jacobi group is defined as $G_{n}^{J}=\mathrm{SL}_{2}(\mathbb{R}) \ltimes H_{n}$ with respect to the action of $\mathrm{SL}_{2}(\mathbb{R})$ on $H_{n}$ given by $\gamma(X, \kappa)=(\gamma X, \kappa)$.

That is,

$$
G_{n}^{J}=\left\{(\gamma, X, \kappa): \gamma \in \mathrm{SL}_{2}(\mathbb{R}), X \in M_{2, n}(\mathbb{R}), \kappa+\frac{1}{2} \iota(X, X) \in \operatorname{Sym}_{n}(\mathbb{R})\right\}
$$

with multiplication given by

$$
(\gamma, X, \kappa)\left(\gamma^{\prime}, X^{\prime}, \kappa^{\prime}\right)=\left(\gamma \gamma^{\prime}, \gamma^{\prime} X+X^{\prime}, \kappa+\kappa^{\prime}-\iota\left(\gamma^{\prime} X, X^{\prime}\right)\right) .
$$

Under the natural inclusion, we can think of $\gamma \in \mathrm{SL}_{2}(\mathbb{R}), X \in M_{2, n}(\mathbb{R})$ and $\kappa \in M_{n}(\mathbb{R})$ as elements of the real Jacobi group $G_{n}^{J}$. In order to avoid confusion with Definition 5.2.2, we write ? for this slash operation of $G_{n}^{J}$. After the definition we explain why this action is well-defined.

Definition 5.3.3. Given an integer $k$ and $M \in M_{n}(\mathbb{Q})$, the real Jacobi group $G_{n}^{J}$ acts on the space of meromorphic functions $\varphi: \mathfrak{h} \times \mathbb{C}^{n} \rightarrow \mathbb{C}$, where the action for $(\gamma, X, \kappa) \in G_{n}^{J}$ is uniquely determined by

(i) $\left(\varphi \imath_{k, M} \gamma\right)(\tau, \boldsymbol{z}):=(c \tau+d)^{-k} \boldsymbol{e}\left(\frac{-c Q_{M}(\boldsymbol{z})}{c \tau+d}\right) \varphi\left(\frac{a \tau+b}{c \tau+d}, \frac{\boldsymbol{z}}{c \tau+d}\right)$;

(ii) $\left(\varphi \imath_{M} X\right)(\tau, \boldsymbol{z}):=\boldsymbol{e}\left(B_{M}(\boldsymbol{\lambda}, 2 \boldsymbol{z}+\boldsymbol{\lambda} \tau+\boldsymbol{\mu})\right) \varphi(\tau, \boldsymbol{z}+\boldsymbol{\lambda} \tau+\boldsymbol{\mu})$;

(iii) $\left(\varphi \imath_{M} \kappa\right)(\tau, \boldsymbol{z}):=\boldsymbol{e}(\langle M, \kappa\rangle) \varphi(\tau, \boldsymbol{z})$,

with $\gamma=\left(\begin{array}{ll}a & b \\ c & d\end{array}\right), X=\left(\begin{array}{l}\boldsymbol{\lambda} \\ \boldsymbol{\mu}\end{array}\right)$ and $\langle M, \kappa\rangle$ the Frobenius inner product of $M$ and $\kappa$, i.e.

$$
\langle M, \kappa\rangle=\sum_{i, j} m_{i, j} \kappa_{i, j} \quad\left(M=\left(m_{i, j}\right)\right) .
$$

Remark 5.3.4. The Frobenius inner product can easily be expressed in terms of the bilinear form, in the following way. For all $M \in M_{n}(\mathbb{Q})$ and $X, X^{\prime} \in M_{2, n}(\mathbb{Q})$ one has by a direct computation that

$$
\left\langle M, \iota\left(X, X^{\prime}\right)\right\rangle=B_{M}\left(\boldsymbol{\lambda}^{\prime}, \boldsymbol{\mu}\right)-B_{M}\left(\boldsymbol{\lambda}, \boldsymbol{\mu}^{\prime}\right),
$$

where $X=\left(\begin{array}{l}\lambda \\ \mu\end{array}\right)$ and $X^{\prime}=\left(\begin{array}{l}\lambda^{\prime} \\ \mu^{\prime}\end{array}\right)$. 
We now show that the above action of the real Jacobi group $G_{n}^{J}$ on the space of meromorphic functions $\varphi: \mathfrak{h} \times \mathbb{C}^{n} \rightarrow \mathbb{C}$ by the above slash equation. It is clear that the identity $(1, \mathbf{0}, 0)$ acts trivially. Hence, it suffices to prove the following identities for all $(\gamma, X, \kappa),\left(\gamma^{\prime}, X^{\prime}, \kappa^{\prime}\right) \in G_{n}^{J}$ (some of which are trivial):

1. $\varphi<\gamma<\gamma^{\prime}=\varphi /\left(\gamma \gamma^{\prime}\right)$;

2. $\varphi \imath X \imath X^{\prime}=\varphi \imath\left(X+X^{\prime}\right) \imath-\iota\left(X, X^{\prime}\right)$;

3. $\varphi \imath \kappa \imath \kappa^{\prime}=\varphi \imath\left(\kappa+\kappa^{\prime}\right)$;

4. $\varphi<X<\gamma=\varphi<\gamma<(\gamma X)$;

5. $\varphi \imath \kappa \curlywedge \gamma=\varphi \imath \gamma \prec \kappa$;

6. $\varphi \nmid \kappa \imath X=\varphi \imath X \imath \kappa$.

The first identity follows by observing that the automorphic factor $j(\gamma, z)=c \tau+d$ and the quasimodular factor $w(\gamma, z)=\frac{c}{c \tau+d}$ (where $\gamma=\left(\begin{array}{ll}a & b \\ c & d\end{array}\right)$ ) satisfy

$$
j\left(\gamma \gamma^{\prime}, \tau\right)=j\left(\gamma^{\prime}, \tau\right) j\left(\gamma, \gamma^{\prime} \tau\right), \quad w\left(\gamma \gamma^{\prime}, \tau\right)=j\left(\gamma^{\prime}, \tau\right)^{-2} w\left(\gamma, \gamma^{\prime} \tau\right)+w\left(\gamma^{\prime}, \tau\right) .
$$

The second identity follows from (5.5). The third follows by linearity of the inner product. Writing $X=\left(\begin{array}{l}\boldsymbol{\lambda} \\ \boldsymbol{\mu}\end{array}\right)$ and $\gamma X=\left(\begin{array}{l}\boldsymbol{\lambda} \gamma \\ \boldsymbol{\mu}^{\gamma}\end{array}\right)$, we find that the fourth equation is a consequence of the identity

$$
z \iota_{0} X \imath_{0,0} \gamma=z \iota_{0} \gamma \iota_{0}(\gamma X)
$$

and the fact that

$$
-w(\gamma, \tau) Q_{M}(\boldsymbol{z})+B_{M}\left(\boldsymbol{\lambda}, \boldsymbol{\lambda}(\gamma \tau)+2 \boldsymbol{z} j(\gamma, \tau)^{-1}+\boldsymbol{\mu}\right)
$$

equals

$$
-w(\gamma, \tau) Q_{M}\left(\boldsymbol{z}+\boldsymbol{\lambda}^{\gamma} \tau+\boldsymbol{\mu}^{\gamma}\right)+B_{M}\left(\boldsymbol{\lambda}^{\gamma}, 2 \boldsymbol{z}+\boldsymbol{\lambda}^{\gamma} \tau+\boldsymbol{\mu}^{\gamma}\right)
$$

for all $M \in M_{n}(\mathbb{Q})$. The last two equations follow directly as the slash action with $\kappa$ multiplies $\varphi$ with a constant not depending on $z$ and $\tau$.

Let

$$
C_{\mathbb{Z}}=\left\{(1,0, \kappa) \mid \kappa \in \operatorname{Sym}_{n}(\mathbb{Z})\right\} .
$$

We fix the group homomorphism $\iota: \Gamma_{n}^{J} \rightarrow G_{n}^{J} / C_{\mathbb{Z}}$ given by

$$
(\gamma, X) \mapsto(\gamma, X, 0)
$$

Observe that $C_{\mathbb{Z}}$ acts trivially by the above action in case $Q_{M}$ is an integer quadratic form. Hence, in this case we have an action of $\Gamma_{n}^{J}$ on functions $\mathfrak{h} \times \mathbb{C} \rightarrow \mathbb{C}$. In case $Q_{M}$ is not an integer quadratic form we modify the slash action by a character of $G_{n}^{J}$ : 
Proposition 5.3.5. Given $M \in M_{n}(\mathbb{Q})$, there exist a character $\rho: G_{n}^{J} \rightarrow \mathbb{C}^{\times}$ satisfying $\rho(\gamma)=1$ for $\gamma \in \mathrm{SL}_{2}(\mathbb{Z})$ and such that

$$
(\varphi, g) \mapsto \rho(g) \varphi \imath_{k, M} g
$$

defines an action of $\Gamma_{n}^{J}$ on the space of functions $\varphi: \mathfrak{h} \times \mathbb{C}^{n} \rightarrow \mathbb{C}$. Moreover, when $\kappa \in \operatorname{Sym}_{n}(\mathbb{Z})$ the character $\rho$ is given by

$$
\rho(\gamma, X, \kappa)=\boldsymbol{e}\left(Q_{M}(\boldsymbol{\lambda})-B_{M}(\boldsymbol{\lambda}, \boldsymbol{\mu})+Q_{M}(\boldsymbol{\mu})+\langle M, \kappa\rangle\right),
$$

where $X=\left(\begin{array}{l}\boldsymbol{\lambda} \\ \boldsymbol{\mu}\end{array}\right)$.

Proof. To prove that $\rho$ satisfies (5.6), we first show that $\rho$ is uniquely determined whenever $\kappa \in \operatorname{Sym}_{n}(\mathbb{Z})$. Note that $\rho(\kappa)=\boldsymbol{e}(\langle M, \kappa\rangle)$ for all $\kappa \in \operatorname{Sym}_{n}(\mathbb{Z})$ as $C_{\mathbb{Z}}$ should act trivially. Because of the assumption $\rho(\gamma)=1$, it suffices to show that $\rho$ is uniquely determined on $M_{2, n}(\mathbb{R})$. Now, note that for $\boldsymbol{\lambda} \in \mathbb{R}^{n}$ one has

$$
\rho(\boldsymbol{\lambda}, \mathbf{0})=\rho(\boldsymbol{\lambda}, \mathbf{0}) \rho\left(\left(\begin{array}{ll}
1 & 1 \\
0 & 1
\end{array}\right)\right)=\rho(\boldsymbol{\lambda}, \boldsymbol{\lambda})=\rho(\boldsymbol{\lambda}, \mathbf{0}) \rho(\mathbf{0}, \boldsymbol{\lambda}) \rho(-\iota((\boldsymbol{\lambda}, \mathbf{0}),(\mathbf{0}, \boldsymbol{\lambda}))) .
$$

Hence,

$$
\rho(\boldsymbol{\lambda}, \mathbf{0})=\rho(\boldsymbol{\lambda}, \mathbf{0}) \rho\left(\left(\begin{array}{cc}
0 & 1 \\
-1 & 0
\end{array}\right)\right)=\rho(\mathbf{0}, \boldsymbol{\lambda})=\rho(\iota((\boldsymbol{\lambda}, \mathbf{0}),(\mathbf{0}, \boldsymbol{\lambda}))) .
$$

This shows $\rho$ is uniquely determined on the generators of $M_{2, n}(\mathbb{R})$.

Next, we show that $\rho$ as defined by (5.6) is a group homomorphism $\Gamma_{n}^{J} \rightarrow \mathbb{C}^{\times}$. Observe that

$$
\rho(\kappa) \varphi \imath \kappa=\boldsymbol{e}(2\langle M, \kappa\rangle) \varphi,
$$

so $\rho$ is constant on $C_{\mathbb{Z}}$. Hence, that $\rho$ is a group homomorphism follows directly if we show that $\rho(X)=\rho(\gamma X)$ and $\rho(X) \rho\left(X^{\prime}\right)=\rho\left(X+X^{\prime}\right) \rho\left(-\iota\left(X, X^{\prime}\right)\right)$ for all $\gamma \in \mathrm{SL}_{2}(\mathbb{Z})$ and $X, X^{\prime} \in M_{2, n}(\mathbb{Z})$.

For the first write $\gamma=\left(\begin{array}{ll}a & b \\ c & d\end{array}\right)$. Then we find that $\rho(\gamma X)$ equals $\boldsymbol{e}\left(\left(a^{2}+b^{2}-a b\right) Q_{M}(\boldsymbol{\lambda})+(2 a c+2 b d-a d-b c) B_{M}(\boldsymbol{\lambda}, \boldsymbol{\mu})+\left(c^{2}+d^{2}-c d\right) Q_{M}(\boldsymbol{\mu})\right)$. As not both $a$ and $b$ are even, it follows that $a^{2}+b^{2}-a b \equiv 1 \bmod 2$. Similarly $c^{2}+$ $d^{2}-c d \equiv 1 \bmod 2$. Also $2 a c+2 b d-a c-b c \equiv 1 \bmod 2$ as $a d-b c=1$. Now, as the bilinear form $Q_{M}$ takes values in $\frac{1}{2} \mathbb{Z}$, it follows that $\rho(\gamma X)=\rho(X)$.

The second equation follows from (5.5), namely, using $Q_{M}$ takes half-integral values on integer vector, one has

$$
\begin{aligned}
\rho(X) & \rho\left(X^{\prime}\right) \\
= & \boldsymbol{e}\left(Q_{M}(\boldsymbol{\lambda})-B_{M}(\boldsymbol{\lambda}, \boldsymbol{\mu})+Q_{M}(\boldsymbol{\mu})+Q_{M}\left(\boldsymbol{\lambda}^{\prime}\right)-B_{M}\left(\boldsymbol{\lambda}^{\prime}, \boldsymbol{\mu}^{\prime}\right)+Q_{M}\left(\boldsymbol{\mu}^{\prime}\right)\right) \\
= & \boldsymbol{e}\left(B_{M}\left(\boldsymbol{\lambda}+\boldsymbol{\lambda}^{\prime}, \boldsymbol{\lambda}+\boldsymbol{\lambda}^{\prime}\right)+B_{M}\left(\boldsymbol{\mu}+\boldsymbol{\mu}^{\prime}, \boldsymbol{\mu}+\boldsymbol{\mu}^{\prime}\right)-B_{M}\left(\boldsymbol{\lambda}+\boldsymbol{\lambda}^{\prime}, \boldsymbol{\mu}+\boldsymbol{\mu}^{\prime}\right)+\right. \\
& \left.\quad+B_{M}\left(\boldsymbol{\lambda}, \boldsymbol{\mu}^{\prime}\right)+B_{M}\left(\boldsymbol{\lambda}^{\prime}, \boldsymbol{\mu}\right)\right) \\
= & \rho\left(X+X^{\prime}\right) \rho\left(-\iota\left(X, X^{\prime}\right)\right) .
\end{aligned}
$$


Definition 5.3.6. Given $M \in M_{n}(\mathbb{Q})$, we define the action of $\Gamma_{n}^{J}$ on a function $\varphi$ : $\mathfrak{h} \times \mathbb{C}^{n} \rightarrow \mathbb{C}$ by the above action

$$
\left.\varphi\right|_{k, M} g=\rho(g) \varphi \imath_{k, M} g
$$

under the group homomorphism $\iota: \Gamma_{n}^{J} \rightarrow G_{n}^{J} / C_{\mathbb{Z}}$. Note that this definition coincides with Definition 5.2.2.

Proof of Proposition 5.2.4. This follows directly from the slash action of the real Jacobi group as the to be proven equalities are equivalent to

$$
\begin{aligned}
\varphi \imath X \imath \gamma & =\varphi \imath \gamma \prec \gamma X \\
\varphi \imath X \imath X^{\prime} & =\varphi \imath X^{\prime} \imath X=\zeta_{X, X^{\prime}}\left(\varphi \imath X+X^{\prime}\right) .
\end{aligned}
$$

\subsection{Poles of Jacobi forms}

In contrast to the space of (weakly) holomorphic Jacobi forms, the space of strictly meromorphic Jacobi forms of given index and weight is not finite dimensional. However, the latter space is not far from being finite dimensional. First of all, in contrast to the space of all meromorphic functions, the space of strictly meromorphic Jacobi forms is not a field. Moreover, the poles lie in a finite number of hyperplanes and after fixing finitely many such hyperplanes to contain the poles, the vector space of strictly meromorphic Jacobi forms of given index and weight is finite dimensional, as we will explain in this section.

Given a meromorphic Jacobi form $\varphi$, we write

$$
P_{\varphi}=\left\{(\tau, \boldsymbol{z}) \in \mathfrak{h} \times \mathbb{C}^{n} \mid \varphi \text { is not holomorphic at }(\tau, \boldsymbol{z})\right\}
$$

for the set of poles as well as points of indeterminacy of $\varphi$. We identify two points of $P_{\varphi}$ if they have same image under the projection $\mathfrak{h} \times \mathbb{C}^{n} \rightarrow M_{2, n}(\mathbb{R})$ given by $(\tau, \boldsymbol{\lambda} \tau+\boldsymbol{\mu}) \mapsto\left(\begin{array}{l}\boldsymbol{\lambda} \\ \boldsymbol{\mu}\end{array}\right)$. That is, we define an equivalence relation on $P_{\varphi}$ by saying that $(\tau, \boldsymbol{z}) \sim\left(\tau^{\prime}, \boldsymbol{z}^{\prime}\right)$ whenever, after writing $\boldsymbol{z}=\boldsymbol{\lambda} \tau+\boldsymbol{\mu}$ and $\boldsymbol{z}^{\prime}=\boldsymbol{\lambda}^{\prime} \tau+\boldsymbol{\mu}^{\prime}$ with $\boldsymbol{\lambda}, \boldsymbol{\lambda}^{\prime}, \boldsymbol{\mu}, \boldsymbol{\mu}^{\prime} \in \mathbb{R}^{n}$, one has $\boldsymbol{\lambda}=\boldsymbol{\lambda}^{\prime}$ and $\boldsymbol{\mu}=\boldsymbol{\mu}^{\prime}$. We identify the quotient set $Q_{\varphi}$ with a subset of $M_{2, n}(\mathbb{R})$ by identifying a point of $P_{\varphi}$ with its image under the projection. From the definition of a strictly meromorphic Jacobi form we obtain the factorisation $\mathfrak{h} \times Q_{\varphi} \simeq P_{\varphi}$ of $P_{\varphi}$, given by $\left(\tau,\left(\begin{array}{l}\boldsymbol{\lambda} \\ \boldsymbol{\mu}\end{array}\right)\right) \mapsto(\tau, \boldsymbol{\lambda} \tau+\boldsymbol{\mu})$. Note that $Q_{\varphi}$ is invariant under translation by $M_{2, n}(\mathbb{Z})$.

As an example of how the definition works, we first prove a simple consequence:

Proposition 5.4.1. Let $\varphi$ be a strictly meromorphic Jacobi form of weight $k$ and index 0 . If $\frac{1}{\varphi}$ is also a strictly meromorphic Jacobi form, then $\varphi$ is constant. 
Proof. Let $X \in M_{2, n}(\mathbb{R})$ be given with $X \notin Q_{\varphi}$ and $X \notin Q_{1 / \varphi}$. Write $\boldsymbol{z}(\tau)=$ $(\tau, 1) X$. Then both $\varphi(\tau, \boldsymbol{z}(\tau))$ and $\frac{1}{\varphi(\tau, \boldsymbol{z}(\tau))}$ are holomorphic as a function of $\tau \in \mathfrak{h}$. Hence, as a function of $\tau \in \mathfrak{h}$, both $\varphi(\tau, \boldsymbol{z}(\tau))$ and $\frac{1}{\varphi(\tau, \boldsymbol{z}(\tau))}$ do not admit any zeros. Similarly, it follows that both $\varphi(\tau, \boldsymbol{z}(\tau))$ and $\frac{1}{\varphi(\tau, \boldsymbol{z}(\tau))}$ are holomorphic (as a function of $\tau \in \mathfrak{h}$ ) at the cusps, hence don't admit any zero at the cusps. Hence, $\varphi(\tau, \boldsymbol{z}(\tau))$ doesn't admit any zeros and poles on a compact set, so $\varphi(\tau, \boldsymbol{z})$ is constant as a function of $\tau$. As this holds for almost all $X \in M_{2, n}(\mathbb{R})$, we conclude that $\varphi$ is globally constant.

The following result, which is crucial for the sequel, tells us that the image of $Q_{\varphi}$ in the torus $M_{2, n}(\mathbb{R} / \mathbb{Z})$ consists of finitely many hyperplanes given by linear equations with rational coefficients. In other words, the following result strengthens Theorem $\mathrm{D}$ (the fact that the second conclusion is also true for quasi-Jacobi forms, follows immediately after introducing such functions; see Corollary 5.6.4).

Theorem 5.4.2. Let $\varphi$ be a strictly meromorphic Jacobi form of weight $k$, index $M$ and rank $n$, and let $P_{\varphi} \simeq \mathfrak{h} \times Q_{\varphi}$ be the set of non-holomorphic points as above. Then, we have:

(i) If $X \in Q_{\varphi}$, then $\gamma X \in Q_{\varphi}$ for all $\gamma \in \mathrm{SL}_{2}(\mathbb{Z})$.

(ii) There exist finitely many hyperplanes of the form

$$
s \cdot X \in(u, v)
$$

with $s \in \mathbb{Z}^{n}$ primitive (i.e., with coprime entries) and $u, v \in \mathbb{Q} / \mathbb{Z}$, such that $X \in Q_{\varphi}$ precisely if $X$ lies on such a hyperplane.

Proof. (i): Let $X \in Q_{\varphi}$ and $\gamma=\left(\begin{array}{ll}a & b \\ c & d\end{array}\right) \in \mathrm{SL}_{2}(\mathbb{Z})$ be given. Write $\boldsymbol{x}(\tau)=(\tau, 1) X$. Then, by the modular transformation behaviour for $\gamma^{-1}$, it follows that

$$
\varphi\left(\frac{d \tau-c}{-b \tau+a}, \frac{\boldsymbol{z}}{-c \tau+a}\right)
$$

has a pole at $\boldsymbol{z}=\boldsymbol{x}(\tau)$ for generic $\tau \in \mathfrak{h}$. Now, let $\tau^{\prime}=\gamma^{-1} \tau$. Writing $\boldsymbol{z}(\tau)=$ $\boldsymbol{\lambda} \tau+\boldsymbol{\mu}$, we find

$$
\frac{\boldsymbol{z}\left(\gamma \tau^{\prime}\right)}{-c \gamma \tau^{\prime}+a}=\frac{\boldsymbol{\lambda} \gamma \tau^{\prime}+\boldsymbol{\mu}}{-c \gamma \tau^{\prime}+a}=\boldsymbol{\lambda}\left(a \tau^{\prime}+b\right)+\boldsymbol{\mu}\left(c \tau^{\prime}+d\right)=\left(\tau^{\prime}, 1\right) \gamma\left(\begin{array}{l}
\boldsymbol{\lambda} \\
\boldsymbol{\mu}
\end{array}\right) .
$$

Hence, the function $\varphi(\tau, \boldsymbol{z})$ has a pole at $\boldsymbol{z}=(\tau, 1) \gamma X$ for generic $\tau \in \mathfrak{h}$. 
(ii): Let $X=\left(\begin{array}{l}\boldsymbol{\lambda} \\ \boldsymbol{\mu}\end{array}\right) \in Q_{\varphi}$. The set $Q_{\varphi}$ is closed and $(n-1)$-dimensional, hence there is an $Y \in M_{2, n}(\mathbb{R})$ with $Y \notin Q_{\varphi}$ and which is bounded away from $\gamma X$.

First, we treat the case that the rank $n$ is 1 . Then, by Lemma 5.5.2, proven in the next section, both $\lambda$ and $\mu$ are rational. Therefore, we can take $s=1$ and $(u, v)=(\lambda, \mu) \bmod \mathbb{Z}^{2}$.

Next, let $n \geq 2$. By an approximation property proven in the next section, there are non-trivial $s \in \mathbb{Z}^{n}, t \in \mathbb{Z}$ and $\alpha, \beta \in \mathbb{Z}$ with $s \cdot(\alpha \boldsymbol{\lambda}+\beta \boldsymbol{\mu})=t$. Note that the value of $s, t, \alpha$, and $\beta$ is a function of $X$, i.e., for all elements $X \in Q_{\varphi}$ there exists an element $(s, t,(\alpha, \beta)) \in$ Rel giving the relation, where

$$
\operatorname{Rel}=\left(\mathbb{Z}^{n} \backslash\{\mathbf{0}\}\right) \times \mathbb{Z} \times\left(\mathbb{Z}^{2} \backslash\{(0,0)\}\right) .
$$

Identify $M_{2, n}(\mathbb{R})$ with $\mathbb{C}^{n}$ via $X \mapsto \boldsymbol{x}=(\tau, 1) X$. For almost all $X \in Q_{\varphi}$, the conditions of the implicit function theorem are satisfied. In this case there exist an open set $U \subset \mathbb{C}^{n-1}$ containing $\mathbf{0}$, an open neighbourhood $V \subset \mathbb{C}^{n}$ of $\boldsymbol{x}$, and a holomorphic function $g: U \rightarrow V$, such that $z \in V$ is a pole precisely if $z$ lies in the image of $g$. Let $g_{i}$ denote the component functions of $g$, which take values in $\mathbb{C} \simeq \mathbb{R} \tau+\mathbb{R}$. Denote this isomorphism by $\left(\pi_{\tau}, \rho_{\tau}\right)$. For example, taking $\tau=\mathrm{i}$, we find $\pi_{\mathrm{i}}\left(g_{i}\right)=\operatorname{Im}\left(g_{i}\right)$ and $\rho_{\mathrm{i}}\left(g_{i}\right)=\operatorname{Re}\left(g_{i}\right)$. For every $\boldsymbol{u} \in U$ we find a relation of the form

$$
\sum_{i=1}^{n} s_{i}\left(\alpha \pi_{\tau}\left(g_{i}(\boldsymbol{u})\right)+\beta \rho_{\tau}\left(g_{i}(\boldsymbol{u})\right)\right)=t,
$$

where $(\boldsymbol{s}, t,(\alpha, \beta)) \in$ Rel, possibly depending on the choice of $\boldsymbol{u}$. We now show that we can choose $(s, t,(\alpha, \beta)) \in$ Rel such that (5.7) holds for all $\boldsymbol{u} \in U$. Recall that the set of zeros of a non-constant real-analytic function has measure 0 . As $\pi_{\tau}\left(g_{i}\right)$ and $\rho_{\tau}\left(g_{i}\right)$ are real-analytic functions, either (5.7) holds for all $\boldsymbol{u} \in U$, or it holds for a real subspace of $U$ of measure 0 . Now, note that Rel is a countable set, whereas countable many subspaces of measure 0 do not cover $U$. Hence, we find a relation (5.7) which holds for all $\boldsymbol{u} \in U$. By the Cauchy-Riemann equations for the holomorphic functions $g_{i}$ we can 'upgrade' this relation to the statement that $\boldsymbol{s} \cdot \boldsymbol{z}$ takes a constant value $u \tau+v$ in $\mathbb{R} \tau+\mathbb{R}$ for all $\boldsymbol{z} \in g(U)$ (possibly after multiplying $\boldsymbol{s}$ by an integer). Without loss of generality we assume that $\operatorname{gcd}\left(s_{1}, \ldots, s_{n}\right)=1$, if not we scale $s, u$ and $v$ appropriately.

Now, by the Weierstrass preparation theorem, locally around $\boldsymbol{x}$ the set of poles is given by $k$ branches coming together, where each branch is an $(n-1)$-dimensional space given by the zeros of a holomorphic function and $k$ equals the multiplicity of the pole at $\boldsymbol{x}$ (see [CJ12, Lemma 6.1] for the same argument in a different setting). By the previous we know that almost all (hence all) the elements in such a branch satisfy $\boldsymbol{s} \cdot \boldsymbol{x}=u \tau+v$. Write $T$ for $(\mathbb{R} \tau+\mathbb{R}) /(\mathbb{Z} \tau+\mathbb{Z})$. By analytically extending such a local 
branch, we find that all solutions $\boldsymbol{z} \in T$ of $\boldsymbol{s} \cdot \boldsymbol{z}=u \tau+v$ are poles of $\varphi(\tau, \cdot)$ as long as they are in the same connected component as $\boldsymbol{x}$. Because $\operatorname{gcd}\left(s_{1}, \ldots, s_{n}\right)=1$ the solution space of $\boldsymbol{s} \cdot \boldsymbol{z}=u \tau+v$ in $T$ is connected, so that all solutions $\boldsymbol{z}$ of $\boldsymbol{s} \cdot \boldsymbol{z} \equiv u \tau+v \bmod L_{\tau}$ are poles of $\varphi(\tau, \cdot)$ for generic $\tau \in \mathfrak{h}$.

Moreover, $(u, v) \in \mathbb{Q}^{2}$, as otherwise the solution space of $\boldsymbol{s} \cdot \boldsymbol{z}=u \tau+v$ in $T$ is not $(n-1)$-dimensional.

Note that $\boldsymbol{z} \mapsto \varphi(\boldsymbol{z}) \Theta(\boldsymbol{s} \cdot \boldsymbol{z}-u \tau-v)$ has exactly the same poles as $\boldsymbol{z} \mapsto \varphi(\boldsymbol{z})$ except for the poles which are zeros of $\boldsymbol{s} \cdot \boldsymbol{z} \equiv u \tau+v \bmod L_{\tau}$. Hence, the statement now follows inductively. By compactness of $T$ it follows that one can restrict to only finitely many linear functions.

Remark 5.4.3. Note that if $s \cdot X \in(u, v)$ is one of the equations determining $Q_{\varphi}$, then $s \cdot X=\left(u^{\prime}, v^{\prime}\right)$ is another equation whenever $(u, v) \gamma=\left(u^{\prime}, v^{\prime}\right)$ for some $\gamma \in \mathrm{SL}_{2}(\mathbb{Z})$.

Corollary 5.4.4. The vector space of strictly meromorphic Jacobi forms of some weight $k$, index $Q$ and poles $(\tau, \boldsymbol{z})$ only in the hyperplanes of the form

$$
\boldsymbol{s} \cdot \boldsymbol{z} \in u \tau+v+L_{\tau}
$$

with $s \in \mathbb{Z}^{n}$ and $u, v \in \mathbb{Q} / \mathbb{Z}$ is finite dimensional.

Proof. This follows directly from the finite dimensionality of the space of weak Jacobi forms of fixed weight and index, together with the fact that the multiplicity at a pole is bounded by the weight $k$. That is, writing $s_{i} z \in u_{i} \tau-v_{i}+L_{\tau}$ for the hyperplanes in the statement, indexed by $i \in I$, we find that the function $\varphi(\boldsymbol{z}) \prod_{i \in I} \Theta\left(\boldsymbol{s}_{i} \cdot \boldsymbol{z}-u_{i} \tau-v_{i}\right)^{k}$ is a weak Jacobi form with index and weight uniquely determined by $\varphi$ and the $s_{i}$.

\subsection{An approximation lemma}

In this section, we prove the approximation properties that were used in the proof of Theorem 5.4.2(ii). That is, we prove a result indicating when given $z, z^{\prime} \in$ $\mathbb{R}^{n} \tau+\mathbb{R}^{n}$, there exist $\gamma \in \mathrm{SL}_{2}(\mathbb{Z})$ such that $\gamma \boldsymbol{z}$ lies arbitrarily close to $\boldsymbol{z}^{\prime}$. For $X \in M_{m, n}(\mathbb{R})$, write $\|X\|$ for the distance to the closest integer matrix, i.e.,

$$
\|X\|:=\max _{i, j} \min _{\ell \in \mathbb{Z}}\left|X_{i, j}-\ell\right| .
$$

Problem 5.5.1. Given $X, Y \in M_{2, n}(\mathbb{R})$, find a condition on $X$ and $Y$ such that

$$
\forall \epsilon>0 \exists \gamma \in \mathrm{SL}_{2}(\mathbb{Z}):\|\gamma X-Y\|<\epsilon
$$


holds. Suppose such a condition is satisfied, i.e. (5.8) is satisfied, we say $Y$ is $\mathrm{SL}_{2}(\mathbb{Z})$-approximable by $X$.

For example, in case $n=1$ a necessary (but not sufficient) condition is given by $X \notin M_{1,2}(\mathbb{Q})$ :

Lemma 5.5.2. Given $X, Y \in M_{2,1}(\mathbb{R})$ and $X \notin M_{2,1}(\mathbb{Q})$, then

$$
\forall \epsilon>0 \exists \gamma \in \mathrm{SL}_{2}(\mathbb{Z}):\|\gamma X-Y\|<\epsilon .
$$

Proof. Write $X=\left(\begin{array}{l}\lambda \\ \mu\end{array}\right)$. First of all, if $\lambda / \mu$ is irrational, then the stronger statement that the orbit of $\left(\begin{array}{c}\lambda \\ \mu\end{array}\right)$ under $\mathrm{SL}_{2}(\mathbb{Z})$ lies dense in $\mathbb{R}^{2}$ holds (see, e.g., [LN12]). Also, if $\mu=0$ the orbit of $\left(\begin{array}{l}\lambda \\ \mu\end{array}\right)$ lies dense in $\mathbb{R}^{2} / \mathbb{Z}^{2}$ whenever $\lambda$ is irrational.

From now on, we assume both $\lambda$ and $\mu$ are irrational, but with rational ratio. Then,

$$
\left(\begin{array}{ll}
a & b \\
c & d
\end{array}\right)\left(\begin{array}{l}
\lambda \\
\mu
\end{array}\right)=\mu\left(\begin{array}{l}
a \frac{\lambda}{\mu}+b \\
c \frac{\lambda}{\mu}+d
\end{array}\right) .
$$

Note that the matrix on the right-hand side parametrises $N^{-1}(\mathbb{Z} \wedge \mathbb{Z})$, where $N$ is the denominator of $\lambda / \mu$ and $\mathbb{Z} \wedge \mathbb{Z}$ denotes the subset of $\mathbb{Z}^{2}$ consisting of coprime integers. As $\mu$ is irrational, we conclude that the orbit of $\left(\begin{array}{c}\lambda \\ \mu\end{array}\right)$ under $\mathrm{SL}_{2}(\mathbb{Z})$ lies dense in $\mathbb{R}^{2} / \mathbb{Z}^{2}$. Hence, if not both $\lambda$ and $\mu$ are rational, then $\mathrm{SL}_{2}(\mathbb{Z})$-approximability of $Y$ by $X$ holds.

If we replace $\mathrm{SL}_{2}(\mathbb{Z})$ by $M_{m, n}(\mathbb{Z})$, it is well-known that Problem 5.5.1 has an affirmative answer [Kro1884].

Theorem 5.5.3 (Kronecker's theorem on Diophantine approximation). For $X, Y \in$ $M_{m, n}(\mathbb{R})$ it holds that

$$
\forall \epsilon>0 \exists \gamma \in M_{m}(\mathbb{Z}):\|\gamma X-Y\|<\epsilon
$$

if and only if

$$
\boldsymbol{s} \in \mathbb{Z}^{n} \text { with } \boldsymbol{s} \cdot X^{t} \in \mathbb{Z}^{m} \text { implies } \boldsymbol{s} \cdot Y^{t} \in \mathbb{Z}^{m} .
$$

After identifying $z$ with $X \in M_{2, n}(\mathbb{R})$, this is almost the result we are looking for, except that we want to replace $M_{2}(\mathbb{Z})$ by $\mathrm{SL}_{2}(\mathbb{Z})$. This question is being touched upon in for example [Dan75, Gui10, LN12], although the focus in the second and third work is a quantitative version in the size of $\gamma$ whenever $n=1$. The second work already hints on the fact that the condition (5.9) should be altered if one replaces $M_{2}(\mathbb{Z})$ by $\mathrm{SL}_{2}(\mathbb{Z})$. Namely, if $\lambda, \mu$ are coprime integers, then $\gamma\left(\begin{array}{l}\lambda \\ \mu\end{array}\right)$ is a vector of coprime integers for all $\gamma \in \mathrm{SL}_{2}(\mathbb{Z})$. Hence, if $X=\left(\frac{1}{2}, \frac{1}{3}\right)$, then $(0,0)$ is 
not in the orbit of $X$ for $\mathrm{SL}_{2}(\mathbb{Z})$, although it is in the orbit of $X$ for $M_{2}(\mathbb{Z})$. Observe that in this case the smallest $s \in \mathbb{Z}_{\geq 1}$ for which $s \cdot(0,0) \in \mathbb{Z}^{2}$ does not equal the smallest $s \in \mathbb{Z}_{\geq 1}$ for which $s \cdot X \in \mathbb{Z}^{2}$ (i.e., $1 \neq 6$ ), which is formalised in (5.11).

For almost all $X=\left(\begin{array}{c}\boldsymbol{\lambda} \\ \boldsymbol{\mu}\end{array}\right) \in M_{2, n}(\mathbb{R})$ we have that $1, \lambda_{1}, \ldots, \lambda_{n}, \mu_{1}, \ldots, \mu_{n}$ are linearly independent over $\mathbb{Q}$. In this case, and, more generally, for generic $X$ defined below, we show one has a Diophantine approximation theorem for $\mathrm{SL}_{2}(\mathbb{Z})$.

Definition 5.5.4. Given $X \in M_{2, n}(\mathbb{R})$, we say $X$ is generic when for all $\alpha, \beta \in \mathbb{Z}$ not both equal to 0 , and for all $s \in \mathbb{Z}^{n}$ with $s \neq \mathbf{0}$ one has

$$
s \cdot(\alpha \boldsymbol{\lambda}+\beta \boldsymbol{\mu}) \notin \mathbb{Z} .
$$

For $s \in \mathbb{Z}^{n}$ and $X \in M_{2, n}(\mathbb{R})$ with $s \cdot X^{t} \in \mathbb{Z}^{2}$, we write

$$
\left(s, s \cdot X^{t}\right)=\max \left\{N \in \mathbb{Z}: \frac{s}{N} \in \mathbb{Z}^{n}, \frac{s \cdot X^{t}}{N} \in \mathbb{Z}^{2}\right\}
$$

for the greatest common divisor of the entries of $s$ and $s \cdot X^{t}$.

Proposition 5.5.5 (Partial result on Diophantine approximation for $\mathrm{SL}_{2}(\mathbb{Z})$ ). Let $X, Y \in M_{2, n}(\mathbb{R})$. Then, if $X$ is generic one has

$$
\forall \epsilon>0 \exists \gamma \in \mathrm{SL}_{2}(\mathbb{Z}):\|\gamma X-Y\|<\epsilon .
$$

Conversely, if (5.10) holds, then for all $s \in \mathbb{Z}^{n}$ for which $s \cdot X^{t} \in \mathbb{Z}^{2}$ one has that $s \cdot Y^{t} \in \mathbb{Z}^{2}$ and

$$
\left(s, s \cdot X^{t}\right)=\left(s, s \cdot Y^{t}\right) .
$$

Proof. Observe that if $s \cdot X^{t} \in \mathbb{Z}^{2}$ (as in the second part of the statement), then $s \cdot(\gamma X)^{t} \in \mathbb{Z}^{2}$ for all $\gamma \in \mathrm{SL}_{2}(\mathbb{Z})$. Hence, if $s \in \mathbb{Z}^{n}$ such that $s \cdot X^{t} \in \mathbb{Z}^{2}$, then for any $\gamma \in \mathrm{SL}_{2}(\mathbb{Z})$ one has

$$
\|\gamma X-Y\| \geq \frac{1}{n}\left\|s \cdot(\gamma X)^{t}-s \cdot Y^{t}\right\|=\frac{1}{n}\left\|s \cdot Y^{t}\right\| .
$$

Therefore, if (5.10) holds, then for all $s \in \mathbb{Z}^{n}$ with $s \cdot X^{t} \in \mathbb{Z}^{2}$ one has $s \cdot Y^{t} \in \mathbb{Z}^{2}$.

Next, suppose $s \in \mathbb{Z}^{n}$ such that $s \cdot X^{t}, s \cdot Y^{t} \in \mathbb{Z}^{2}$. Write $N=\left(s, s \cdot Y^{t}\right)$ and $\boldsymbol{s}^{\prime}=N^{-1} s$. Suppose $\boldsymbol{s}^{\prime} \cdot(\gamma X)^{t} \notin \mathbb{Z}^{2}$ for all $\gamma \in \mathrm{SL}_{2}(\mathbb{Z})$, i.e., $\boldsymbol{s}^{\prime} \cdot(\gamma X)^{t} \in$ $\left(N^{-1} \mathbb{Z}^{2}\right) \backslash \mathbb{Z}^{2}$. Then, for any $\gamma \in \mathrm{SL}_{2}(\mathbb{Z})$ one has

$$
\|\gamma X-Y\| \geq \frac{1}{n}\left\|s \cdot(\gamma X)^{t}-s \cdot Y^{t}\right\|=\frac{1}{n}\left\|s \cdot(\gamma X)^{t}\right\| \geq \frac{1}{N n} .
$$


Therefore, if (5.10) holds, we have that $s^{\prime} \cdot(\gamma X)^{t} \in \mathbb{Z}^{2}$ for some $\gamma \in \mathrm{SL}_{2}(\mathbb{Z})$. This implies that $s^{\prime} \cdot X^{t} \in \mathbb{Z}^{2}$. Together with the previous part, we conclude that $\left(s, s \cdot X^{t}\right)=\left(s, s \cdot Y^{t}\right)$.

Next, let $X=\left(\begin{array}{l}\lambda \\ \mu\end{array}\right)$ be generic and $\epsilon>0$. In three steps we construct a matrix $\gamma \in \mathrm{SL}_{2}(\mathbb{Z})$ for which $\|\gamma X-Y\|<\epsilon$. First we construct a matrix $\gamma_{1} \in M_{2}(\mathbb{Z})$ for which $\|\gamma X-Y\|$ is small. Next, we find another $\gamma_{2} \in M_{2}(\mathbb{Z})$ with the additional property that the entries in the first row are coprime. Finally, in the third step, we modify $\gamma_{2}$ and find $\gamma_{3} \in M_{2}(\mathbb{Z})$ of determinant equal to 1 , i.e., $\gamma_{3} \in \mathrm{SL}_{2}(\mathbb{Z})$.

First step. As $X$ is generic, there is no $s \in \mathbb{Z}^{n}$ such that $s \cdot X^{t} \in \mathbb{Z}^{2}$. Hence, by Kronecker's theorem we find $\gamma_{1}=\left(\begin{array}{ll}a_{1} & b_{1} \\ c_{1} & d_{1}\end{array}\right) \in M_{2}(\mathbb{Z})$ such that $\left\|\gamma_{1} X-Y\right\|<\frac{1}{3} \epsilon$.

Second step. Let $k \in \mathbb{Z}$ be such that

$$
\left\|\left(k a_{1}-b_{1}+1\right) \boldsymbol{\mu}\right\|<\frac{1}{3} \epsilon,
$$

whose existence is guaranteed for generic $X$ by Kronecker's theorem. Then,

$$
\gamma_{2}:=\left(\begin{array}{ll}
a_{2} & b_{2} \\
c_{2} & d_{2}
\end{array}\right):=\gamma_{1}+\left(\begin{array}{cc}
0 & k a_{1}-b_{1}+1 \\
0 & 0
\end{array}\right)
$$

satisfies

$$
\left\|\gamma_{2} X-Y\right\| \leq\left\|\gamma_{1} X-Y\right\|+\left\|\left(k a_{1}-b_{1}+1\right) \boldsymbol{\mu}\right\|<\frac{2}{3} \epsilon .
$$

As $a_{2}=a_{1}$ and $b_{2}=k a_{1}+1$, clearly $\left(a_{2}, b_{2}\right)=1$.

Third step. Let $\tilde{c}$ and $\tilde{d}$ be given by the inverse Euclidean algorithm such that $a_{2} \tilde{d}-b_{2} \tilde{c}=1$. We let $\ell \in \mathbb{Z}$ be such that

$$
\left\|\left(\ell a_{2}+\tilde{c}-c_{2}\right) \boldsymbol{\lambda}+\left(\ell b_{2}+\tilde{d}-d_{2}\right) \boldsymbol{\mu}\right\|<\frac{1}{3} \epsilon,
$$

whose existence is again a consequence of $X$ being generic. Then, let

$$
\gamma_{3}=\gamma_{2}+\left(\begin{array}{cc}
0 & 0 \\
\ell a_{2}+\tilde{c}-c_{2} & \ell b_{2}+\tilde{d}-d_{2}
\end{array}\right) .
$$

Invoking the triangle inequality again, we conclude

$$
\left\|\gamma_{3} X-Y\right\| \leq \epsilon
$$

Moreover, det $\gamma_{3}=a_{2}\left(\ell b_{2}+\tilde{d}\right)-b_{2}\left(\ell a_{2}+\tilde{c}\right)=1$, which concludes the proof.

Remark 5.5.6. Condition (5.11) is necessary for $\mathrm{SL}_{2}(\mathbb{Z})$-approximability of $Y$ by $X$. Whether this condition also suffices, or, if not, how it should be strengthened to a necessary and sufficient condition remains an open problem. 


\subsection{Quasi-Jacobi forms}

We weaken the notion of a strictly meromorphic Jacobi form even further by introducing the real-analytic functions

$$
\nu(\tau)=\frac{1}{2 \mathrm{i} \operatorname{Im}(\tau)}, \quad \xi(\tau, z)=\frac{\operatorname{Im}(z)}{\operatorname{Im}(\tau)},
$$

which almost transform as a Jacobi form of index 0 and weight 1 and 2 respectively:

$$
\begin{aligned}
\left(\left.\nu\right|_{2,0} \gamma\right)(\tau) & =\nu(\tau)-\frac{c}{c \tau+d} & \left(\left.\xi\right|_{1,0} \gamma\right)(\tau, z) & =\xi(\tau, z)-\frac{c z}{c \tau+d} \\
\left(\left.\nu\right|_{0} X\right)(\tau) & =\nu(\tau) & \left(\left.\xi\right|_{0} X\right)(z) & =\xi(z)+\lambda .
\end{aligned}
$$

Definition 5.6.1. Let $k \in \mathbb{Z}, M \in M_{n}(\mathbb{Q})$. Denote by $C$ a subspace of all strictly meromorphic functions $\mathfrak{h} \times \mathbb{C}^{n} \rightarrow \mathbb{C}$. An almost Jacobi form $\Phi$ of analytic type $C$, weight $k$, index $Q$ and rank $n$ satisfies:

(i) $\Phi \in C\left[\nu(\tau), \xi\left(\tau, z_{1}\right), \ldots, \xi\left(\tau, z_{n}\right)\right]$;

(ii) $\Phi \mid g=\Phi$ for all $g \in \Gamma_{n}^{J}$.

A quasi-Jacobi form $\varphi$ is the constant term with respect to $\nu$ and $\xi$ of an almost Jacobi form. If $C$ equals $\mathrm{Hol}_{n}^{M}, \mathrm{Hol}_{n}$ or $\mathrm{Mer}_{n}^{M}, \varphi$ is a holomorphic, weak or strictly meromorphic quasi-Jacobi form, respectively.

As a first example, quasimodular forms are quasi-Jacobi forms of rank $n=0$. More interestingly, the functions

$$
E_{2}(\tau, z)-2 \pi \mathrm{i} \nu(\tau), \quad E_{1}(\tau, z)+2 \pi \mathrm{i} \xi(\tau, z),
$$

with $E_{1}$ and $E_{2}$ defined by regularizing the sum (5.1) as in [Wei99], are almost strictly meromorphic Jacobi forms of index 0 and weight 2 and 1 respectively, so that $E_{2}$ and $E_{1}$ are strictly meromorphic quasi-Jacobi forms. Let $D_{z}=\frac{1}{2 \pi \mathrm{i}} \frac{\partial}{\partial z}$. Observe that

$$
E_{2}(\tau, z)=-D_{z} E_{1}(\tau, z) \quad E_{1}(\tau, z)=(2 \pi \mathrm{i}) D_{z} \log \Theta(\tau, z),
$$

which reminds one of

$$
e_{2}=8 \pi^{2} D_{\tau} \log \eta
$$

where $\eta(\tau)=q^{1 / 24} \prod_{n}\left(1-q^{n}\right)$ and $D_{\tau}=\frac{1}{2 \pi \mathrm{i}} \frac{\partial}{\partial \tau}$. The strictly meromorphic quasiJacobi forms $e_{2}$ and $E_{1}$ play a central role as building blocks of quasi-Jacobi forms 
out of Jacobi forms. For convenience, we introduce the following alternative normalisations

$$
\mathbb{e}_{2}:=\frac{1}{4 \pi^{2}} e_{2}=\frac{1}{12}-2 \sum_{m, r \geq 1} m q^{m r}, \quad A=\frac{1}{2 \pi \mathrm{i}} E_{1} .
$$

Quasi-Jacobi forms are by construction strictly meromorphic, but they are not invariant under the action of the Jacobi group. However, the fact that an almost Jacobi form is a polynomial in $\nu$ and $\xi$ implies that a quasi-Jacobi form transforms "up to a polynomial correction" as a Jacobi form, or, equivalently, that it is a polynomial in the strictly meromorphic quasi-Jacobi forms $\mathbb{E}_{2}(\tau)$ and $A(\tau, z)$ with Jacobi forms as coefficients.

Recall that for vectors $\boldsymbol{a} \in \mathbb{C}^{n}, \boldsymbol{b} \in \mathbb{Z}^{n}$ we write $\boldsymbol{a}^{\boldsymbol{b}}=\prod_{r} a_{r}^{b_{r}}$.

Proposition 5.6.2. Two equivalent definitions for a strictly meromorphic quasi-Jacobi form are as follows: a strictly meromorphic quasi-Jacobi form is a meromorphic function $\varphi: \mathfrak{h} \times \mathbb{C}^{n} \rightarrow \mathbb{C}$ with $\varphi \in \operatorname{Mer}_{n}^{M}$ for which there exist a finite number of

(1) functions $\varphi_{i, j} \in \operatorname{Mer}_{n}^{M}$ satisfying

$$
\begin{aligned}
(\varphi \mid \gamma)(\tau, \boldsymbol{z}) & =\sum_{i, \boldsymbol{j}} \varphi_{i, \boldsymbol{j}}(\tau, \boldsymbol{z})\left(\frac{c}{c \tau+d}\right)^{i+|\boldsymbol{j}|} \boldsymbol{z}^{\boldsymbol{j}} \\
(\varphi \mid X)(\boldsymbol{z}) & =\sum_{\boldsymbol{j}} \varphi_{0, \boldsymbol{j}}(\boldsymbol{z})(-\boldsymbol{\lambda})^{\boldsymbol{j}}
\end{aligned}
$$

(2) strictly meromorphic Jacobi forms $\psi_{i, j}$ satisfying

$$
\varphi(\boldsymbol{z})=\sum_{i, \boldsymbol{j}} \psi_{i, \boldsymbol{j}}(\boldsymbol{z}) \mathbb{e}_{2}^{i} A\left(z_{1}\right)^{j_{1}} \cdots A\left(z_{n}\right)^{j_{n}} .
$$

Proof. The first part follows directly from the definition of quasi-Jacobi forms using the algebraic independence of $\nu$ and $\xi$ over the field of meromorphic functions, where $\varphi_{i, j}$ are given by the meromorphic almost Jacobi form $\Phi$ corresponding to $\varphi$ by

$$
\Phi(\tau, \boldsymbol{z})=\sum_{i, \boldsymbol{j}} \varphi_{i, \boldsymbol{j}}(\tau, \boldsymbol{z}) \nu(\tau)^{i} \xi\left(\tau, z_{1}\right)^{j_{1}} \cdots \xi\left(\tau, z_{n}\right)^{j_{n}} .
$$

For the second part we define the coefficients $\psi_{i, j}$ by the expansion of the almost Jacobi form $\Phi$ corresponding to $\varphi$, i.e.,

$$
\Phi(\tau, \boldsymbol{z})=\sum_{i, \boldsymbol{j}} \psi_{i, \boldsymbol{j}}(\tau, \boldsymbol{z})\left(\mathbb{e}_{2}(\tau)+\frac{\nu(\tau)}{2 \pi \mathrm{i}}\right)^{i} \prod_{r}\left(A\left(\tau, z_{r}\right)+\xi\left(\tau, z_{r}\right)\right)^{j_{r}} .
$$


Note that $\mathbb{e}_{2}(\tau)+\frac{\nu(\tau)}{2 \pi \mathrm{i}}$ and $A\left(\tau, z_{r}\right)+\xi\left(\tau, z_{r}\right)$ transform as Jacobi forms. Moreover, they are algebraically independent over the space of all meromorphic functions. Hence, it follows that the coefficients $\psi_{i, j}$ are strictly meromorphic Jacobi forms. The constant term with respect to $i$ and $\boldsymbol{j}$, by definition equal to $\varphi$, is now easily seen to equal to right-hand side of (5.15).

Remark 5.6.3. In fact, the proof implies that $\varphi_{i, j}$ are quasi-Jacobi forms related to the $\psi_{i, j}$ by

$$
(2 \pi \mathrm{i})^{i} \varphi_{i, \boldsymbol{j}}=\sum_{i^{\prime}, \boldsymbol{j}^{\prime}} \psi_{i+i^{\prime}, \boldsymbol{j}+\boldsymbol{j}^{\prime}}\left(\begin{array}{c}
i+i^{\prime} \\
i
\end{array}\right)\left(\begin{array}{c}
\boldsymbol{j}+\boldsymbol{j}^{\prime} \\
\boldsymbol{j}
\end{array}\right) \mathbb{e}_{2}(\tau)^{i^{\prime}} \prod_{r} A\left(\tau, z_{r}\right)^{j_{r}^{\prime}},
$$

where

$$
\left(\begin{array}{c}
\boldsymbol{j}+\boldsymbol{j}^{\prime} \\
\boldsymbol{j}
\end{array}\right)=\prod_{r}\left(\begin{array}{c}
j_{r}+j_{r}^{\prime} \\
j_{r}
\end{array}\right) .
$$

Also, note that, given a representation for $\varphi$ as in (5.15) one has

$$
\varphi_{1,0}=\frac{1}{2 \pi \mathrm{i}} \frac{\partial}{\partial \mathbb{e}_{2}} \varphi \quad \text { and } \quad \varphi_{0, e_{i}}(\boldsymbol{z})=\frac{\partial}{\partial A\left(z_{i}\right)} \varphi(\boldsymbol{z}) .
$$

By virtue of (5.15) quasi-Jacobi forms share the properties of Jacobi forms with respect to the location of the poles:

Corollary 5.6.4. The statement of Theorem 5.4.2 also holds when $\varphi$ is a strictly meromorphic quasi-Jacobi form.

Another corollary of Proposition 5.2.8 is the following. Denoting by $J^{0}$ and $\widetilde{J}^{0}$ the space of all strictly meromorphic Jacobi forms and strictly meromorphic quasiJacobi forms, respectively, with all poles at the lattice points $L_{\tau}$, we have the following representations.

$$
J^{0}=\mathbb{C}\left[E_{2}-e_{2}, E_{3}, e_{4}, \Theta\right], \quad \widetilde{J}^{0}=\mathbb{C}\left[E_{1}, E_{2}, E_{3}, e_{2}, e_{4}, \Theta\right] .
$$

In particular, given the weight and index, these spaces are finite dimensional.

Corollary 5.6.5. The space of all meromorphic quasi-Jacobi forms of weight $k$, in$\operatorname{dex} M$, and with all poles in a finite union of rational hyperplanes as in Theorem $D$ is finite dimensional.

Proof. This follows directly from the previous proposition as by Corollary 5.4.4 the number of linearly independent $\psi_{i, j}$ is finite. 


\subsection{Action of the Jacobi Lie algebra by derivations}

The operators $D_{\tau}=\frac{1}{2 \pi \mathrm{i}} \frac{\partial}{\partial \tau}$ and $D_{z_{i}}=\frac{1}{2 \pi \mathrm{i}} \frac{\partial}{\partial z_{i}}$ preserve the space of quasi-Jacobi forms. These operators are part of a Lie algebra of operators acting on quasi-Jacobi forms by derivations, as we explain now.

Following a suggestion by Zagier, we consider the notion of a $\mathfrak{g}$-algebra for any Lie algebra $\mathfrak{g}$ given by the following definition, which specializes to Definition 1.2.4 if $\mathfrak{g}=\mathfrak{s l}_{2}$.

Definition 5.7.1. Given a Lie algebra $\mathfrak{g}$, a $\mathfrak{g}$-algebra is an algebra $A$ together with a Lie homomorphism $\mathfrak{g} \rightarrow \operatorname{Der}(A)$, where $\operatorname{Der}(A)$ denotes the Lie algebra of all derivations on $A$.

Denote by $\mathfrak{d}_{\tau}$ and $\mathfrak{d}_{z_{i}}$ the operators on the space of quasi-Jacobi forms given by $\varphi \mapsto 2 \pi \mathrm{i} \varphi_{1,0}$ and $\varphi \mapsto \varphi_{0, e_{i}}$ respectively (see Proposition 5.6.2 and Remark 5.6.3). Then the functions $\varphi_{i, j}$ are given by

$$
\varphi_{i, j}=\frac{\mathfrak{d}_{\tau}^{i}}{i !} \frac{\mathfrak{d}_{z}^{j}}{\boldsymbol{j} !} \varphi \quad\left(\mathfrak{d}_{z}^{j}=\mathfrak{d}_{z_{1}}^{j_{1}} \cdots \mathfrak{d}_{z_{n}}^{j_{n}}, \boldsymbol{j} !=j_{1} ! \cdots j_{n} !\right),
$$

so that the transformation behaviour of $\varphi$ is uniquely determined by the action of the operators $\mathfrak{d}_{\tau}$ and $\mathfrak{d}_{z_{i}}$. This observation is important to understand the next section, where we investigate how the transformation behaviour of a quasi-Jacobi form determines the transformation of its Taylor coefficients, and vice versa, by studying the action of $\mathfrak{d}_{\tau}$ on these Taylor coefficients.

Moreover, writing $\varphi$ as in (5.15) yields

$$
\mathfrak{d}_{\tau} \varphi=\frac{\partial}{\partial \mathbb{e}_{2}} \varphi, \quad\left(\mathfrak{d}_{z_{i}} \varphi\right)(\boldsymbol{z})=\frac{\partial}{\partial A\left(z_{i}\right)} \varphi\left(z_{i}\right) .
$$

Denote by $W$ and $I_{i j}$ the weight and index operators acting diagonally by multiplying with the weight $k$ and $Q\left(e_{i}, e_{j}\right)$ respectively, where $Q$ is the index. Let $\mathfrak{j}$ the Lie algebra of the Jacobi group. By [OP19, Eqn. (12)] the Lie algebra of the Jacobi group acts by the beforementioned operators on the space of quasi-Jacobi forms.

Proposition 5.7.2. The algebra of quasi-Jacobi forms is a j-algebra, i.e., the algebra of derivations $D_{\tau}, D_{z_{i}}, \mathfrak{d}_{\tau}, \mathfrak{d}_{z_{i}}, W$ and $I_{i j}$ is isomorphic to $\mathfrak{j}$ and acts on the space of quasi-Jacobi forms.

Remark 5.7.3. More concretely, the commutation relations of (i) the modular operators, (ii) the elliptic operators and (iii) their interactions are given by

$$
\begin{aligned}
{\left[\mathfrak{d}_{\tau}, D_{\tau}\right] } & =W, & {\left[W, D_{\tau}\right] } & =2 D_{\tau}, & {\left[W, \mathfrak{d}_{\tau}\right] } & =-2 \mathfrak{d}_{\tau}, \\
{\left[\mathfrak{d}_{z_{i}}, D_{z_{j}}\right] } & =2 I_{i, j}, & {\left[I_{i j}, D_{z_{i}}\right] } & =0, & {\left[I_{i j}, \mathfrak{d}_{z_{i}}\right] } & =0, \\
{\left[\mathfrak{d}_{z_{i}}, D_{\tau}\right] } & =D_{z_{i}}, & {\left[\mathfrak{d}_{\tau}, D_{z_{i}}\right] } & =\mathfrak{d}_{z_{i}}, & {\left[W, D_{z_{i}}\right] } & =D_{z_{i}} .
\end{aligned}
$$


The other commutators vanish. As the spaces of almost Jacobi forms and quasiJacobi forms are isomorphic, the same result holds for almost Jacobi forms when one replaces $\mathfrak{d}_{\tau}$ by $2 \pi \mathrm{i} \frac{\partial}{\partial \nu}$ and $\mathfrak{d}_{z}$ by $\frac{\partial}{\partial \xi(z)}$.

There is yet another equivalent definition of quasi-Jacobi forms as derivatives of Jacobi forms. This definition only applies in case the index is positive definite. As a non-example, no power of the quasi-Jacobi form $e_{2}$ (which is in fact a quasimodular form) can be written in terms of derivatives of Jacobi forms.

Proposition 5.7.4. Let $\varphi$ be a quasi-Jacobi form of weight $k$ and positive definite index $M$. Then, there exist unique Jacobi forms $\psi_{\boldsymbol{d}}$ with $\boldsymbol{d} \in \mathbb{Z}_{\geq 0}^{n+1}$ of weight $k-$ $2 d_{0}-d_{1}-\ldots-d_{n}$ and index $M$ such that

$$
\varphi=\sum_{\boldsymbol{d}} D_{\tau}^{d_{0}} D_{z_{1}}^{d_{1}} \cdots D_{z_{n}}^{d_{n}} \psi_{\boldsymbol{d}} .
$$

Proof. Choose an ordering on $\mathbb{Z}^{n+1}$ respecting the ordering on $\mathbb{Z}$. Given a Jacobi form $\varphi$, let $(i, \boldsymbol{j})$ be maximal (with respect to this ordering) for which $\varphi_{i, \boldsymbol{j}}$ in Proposition 5.6.2 exists and is non-zero. A direct check using the same proposition shows that $\varphi$ minus a multiple of $D_{\tau}^{j_{0}} D_{z_{j_{1}}}^{j_{1}} \cdots D_{z_{j_{n}}}^{j_{n}} \varphi_{i, j}$ is a quasi-Jacobi form for which this maximal index is smaller. Here, by the positive definiteness of $Q$ this multiple is non-zero.

\subsection{The double slash operator}

A holomorphic Jacobi form has two important representations: the theta expansion and the Taylor expansion. We generalise the Taylor expansion to strictly meromorphic quasi-Jacobi forms in such a way that the Taylor coefficients are quasimodular forms. Moreover, we give criteria based on the coefficients in these representations for a meromorphic function to be a quasi-Jacobi form.

Given a Jacobi form $\varphi$ and $X \in M_{2, n}(\mathbb{Q})$, the Taylor coefficients of $(\varphi \mid X)(z)$ around $\boldsymbol{z}=\mathbf{0}$ are quasimodular forms for the group

$$
\Gamma_{X}=\left\{\gamma \in \mathrm{SL}_{2}(\mathbb{Z}) \mid \gamma X-X \in M_{2, n}(\mathbb{Z}), \rho(X-\gamma X)=\zeta_{X, \gamma X-X}\right\},
$$

where as usual $X=\left(\begin{array}{l}\boldsymbol{\lambda} \\ \boldsymbol{\mu}\end{array}\right)$ and $\gamma X=\left(\begin{array}{l}\boldsymbol{\lambda}_{\boldsymbol{\mu}}^{\gamma} \\ \boldsymbol{\mu}^{\gamma}\end{array}\right)$ and $\rho$ and $\zeta_{X, X^{\prime}}$ are defined by (5.4). In contrast to Jacobi forms, it is not true that the Taylor coefficients of quasi-Jacobi forms are quasimodular. Namely, as stated in the introduction, for $X=\left(\begin{array}{l}\lambda \\ \mu\end{array}\right) \in$ $M_{2, n}(\mathbb{Q})$ one has that $\left(\Theta^{\prime}|X| \gamma\right)(\tau, z)$ equals

$$
\left(\Theta^{\prime} \mid \gamma X\right)(\tau, z)+\frac{c z}{c \tau+d}(\Theta \mid \gamma X)(\tau, z)+\lambda(\Theta \mid \gamma X)(\tau, z)-\frac{\lambda}{c \tau+d}(\Theta \mid \gamma X)(\tau, z),
$$


up to the multiplicative constant $\rho(X) \rho(-\gamma X)$, for all $\gamma \in \Gamma_{X}$. All but the last term $-\frac{\lambda}{c \tau+d}(\Theta \mid X)(\tau, z)$ of (5.18) depend polynomially on $\frac{c}{c \tau+d}$, so that the Taylor coefficients of $\left(\Theta^{\prime} \mid X\right)(\tau, \boldsymbol{z})$ at $\boldsymbol{z}=0$ are not transforming in accordance with the quasimodular transformation formula. Note the this last term can be written as $-\lambda\left(\Theta|X|_{0} \gamma\right)(\tau, z)$, where it should be noted that the weight 0 in the slash operator is unusual, corresponding to $\Theta^{\prime}$ rather than to $\Theta$. In conclusion, the function

$$
\Theta^{\prime} \| X:=\rho(-X)\left(\Theta^{\prime}|X+\lambda \Theta| X\right)
$$

rather than $\Theta^{\prime} \mid X$ transforms as a quasi-Jacobi form of weight 0 , i.e., for $\gamma \in \Gamma_{X}$ one has

$$
\left(\Theta^{\prime} \|\left. X\right|_{0} \gamma\right)(\tau, z)=\left(\Theta^{\prime} \| X\right)(\tau, z)+\frac{c z}{c \tau+d}(\Theta \mid X)(\tau, z) .
$$

Therefore, when we define the Taylor coefficients of a function $\varphi$ at $X \in M_{2, n}(\mathbb{Q})$ in the next section, we in fact take the usual Taylor coefficients of $(\varphi \| X)(\tau, \boldsymbol{z})$, defined in the definition below, around $\boldsymbol{z}=\mathbf{0}$.

Definition 5.8.1. Given $M \in M_{n}(\mathbb{Q})$ and a family of functions $\varphi_{0, j}: \mathfrak{h} \times \mathbb{C}^{n} \rightarrow \mathbb{C}$ indexed by a finite subset of $\mathbb{Z}_{\geq 0}^{n}$ (with $\varphi:=\varphi_{0,0}$ ), define the double slash operator by

$$
\varphi \|_{M} X=\rho(-X) \sum_{j}\left(\left.\varphi_{0, j}\right|_{M} X\right) \lambda^{j},
$$

where $\rho$ is given by (5.4).

Convention 5.8.2. In case $\varphi$ is a quasi-Jacobi form, in this definition we always take the family $\varphi_{0, j}$ determined by the elliptic transformation (5.15).

Proposition 5.8.3. Given a family of functions $\varphi_{i, j}: \mathfrak{h} \times \mathbb{C}^{n} \rightarrow \mathbb{C}$ indexed by a finite subset of $\mathbb{Z}_{\geq 0} \times \mathbb{Z}_{\geq 0}^{n}$ (with $\varphi=\varphi_{0,0}$ ) and $X \in M_{2, n}(\mathbb{R})$, one has

(i) If $\varphi$ satisfies the quasimodular transformation (5.13) for $\Gamma$, then

$$
(\varphi \| X \mid \gamma)(\tau, \boldsymbol{z})=\sum_{i, \boldsymbol{j}}\left(\varphi_{i, \boldsymbol{j}} \| \gamma X\right)(\tau, \boldsymbol{z})\left(\frac{c}{c \tau+d}\right)^{i+|\boldsymbol{j}|} \boldsymbol{z}^{\boldsymbol{j}}
$$

for all $\gamma \in \Gamma$.

(ii) If $\varphi$ satisfies the quasi-elliptic transformation (5.14), then

$$
\varphi\|X\| X^{\prime}=\varphi\left\|X^{\prime}\right\| X=\rho\left(X^{\prime}\right) \varphi\|X, \quad \varphi\| X+X^{\prime}=\zeta_{X, X^{\prime}} \varphi\|X\| X^{\prime}
$$

for all $X^{\prime} \in M_{2, n}(\mathbb{Z})$, where the root of unity $\zeta_{X, X^{\prime}}$ is defined by (5.4). 
(iii) If $\varphi$ is a quasi-Jacobi form for $\mathrm{SL}_{2}(\mathbb{Z})$, then $\varphi \| X$ is a quasi-Jacobi form for $\Gamma_{X}$, and

$$
\varphi\left\|X+X^{\prime}=\rho\left(X^{\prime}\right) \zeta_{X, X^{\prime}} \varphi\right\| X
$$

for all $X^{\prime} \in M_{2, n}(\mathbb{Z})$.

Proof. The transformation of $\varphi \| X$ under $\gamma$ and $X$ follows by direct computations.

We often make use of

$$
\frac{\mathfrak{d}_{\tau}^{i^{\prime}}}{i^{\prime} !} \frac{\mathfrak{d}_{z}^{j^{\prime}}}{\boldsymbol{j}^{\prime !}} \varphi_{i, \boldsymbol{j}}=\frac{\mathfrak{d}_{\tau}^{i^{\prime}}}{i^{\prime} !} \frac{\mathfrak{d}_{z}^{j^{\prime}}}{\boldsymbol{j}^{\prime} !} \frac{\mathfrak{d}_{\tau}^{i}}{i !} \frac{\mathfrak{d}_{z}^{j}}{\boldsymbol{j} !} \varphi=\left(\begin{array}{c}
i+i^{\prime} \\
i
\end{array}\right)\left(\begin{array}{c}
\boldsymbol{j}+\boldsymbol{j}^{\prime} \\
\boldsymbol{j}
\end{array}\right) \varphi_{i+i^{\prime}, \boldsymbol{j}+\boldsymbol{j}^{\prime}}
$$

where

$$
\left(\begin{array}{c}
\boldsymbol{j}+\boldsymbol{j}^{\prime} \\
\boldsymbol{j}
\end{array}\right)=\prod_{r}\left(\begin{array}{c}
j_{r}+j_{r}^{\prime} \\
j_{r}
\end{array}\right)
$$

For the first property:

$$
\begin{aligned}
\varphi \|\left. X\right|_{k} \gamma & =\rho(-X) \sum_{\ell}\left(\varphi_{0, \ell}|X|_{k} \gamma\right) \boldsymbol{\lambda}^{\ell} \\
& =\rho(-\gamma X) \sum_{\ell}\left(\left.\varphi_{0, \ell}\right|_{k} \gamma \mid \gamma X\right) \boldsymbol{\lambda}^{\ell} \\
& =\rho(-\gamma X) \sum_{i, \boldsymbol{j}}\left(\varphi_{i, \boldsymbol{j}} \mid \gamma X\right)\left(\frac{c}{c \tau+d}\right)^{i}\left(\frac{c\left(\boldsymbol{z}+\boldsymbol{\lambda}^{\gamma} \tau+\boldsymbol{\mu}^{\gamma}\right)+\boldsymbol{\lambda}}{c \tau+d}\right)^{j} \\
& =\rho(-\gamma X) \sum_{i, \boldsymbol{j}}\left(\varphi_{i, \boldsymbol{j}} \mid \gamma X\right)\left(\frac{c}{c \tau+d}\right)^{i}\left(\frac{c \boldsymbol{z}}{c \tau+d}+\boldsymbol{\lambda}^{\gamma}\right)^{j} \\
& =\rho(-\gamma X) \sum_{i, \boldsymbol{j}, \boldsymbol{\ell}}\left(\varphi_{i, \boldsymbol{j}+\ell} \mid \gamma X\right)\left(\frac{c}{c \tau+d}\right)^{i}\left(\begin{array}{c}
\boldsymbol{j}+\boldsymbol{\ell} \\
\boldsymbol{j}
\end{array}\right)\left(\frac{c \boldsymbol{z}}{c \tau+d}\right)^{\boldsymbol{j}}\left(\boldsymbol{\lambda}^{\gamma}\right)^{\boldsymbol{\ell}} \\
& =\sum_{i, \boldsymbol{j}}\left(\varphi_{i, \boldsymbol{j}} \| \gamma X\right)\left(\frac{c}{c \tau+d}\right)^{i}\left(\frac{c \boldsymbol{z}}{c \tau+d}\right)^{\boldsymbol{j}} .
\end{aligned}
$$

For the second property, observe that

$$
\begin{aligned}
\varphi\|X\| X^{\prime} & =\rho\left(X^{\prime}\right) \sum_{\ell}\left(\varphi_{0, \ell} \| X \mid X^{\prime}\right)\left(\boldsymbol{\lambda}^{\prime}\right)^{\ell} \\
& =\rho(X) \rho\left(X^{\prime}\right) \sum_{\boldsymbol{j}, \boldsymbol{\ell}}\left(\varphi_{0, \boldsymbol{j}+\boldsymbol{\ell}}|X| X^{\prime}\right)\left(\begin{array}{c}
\boldsymbol{j}+\boldsymbol{\ell} \\
\boldsymbol{j}
\end{array}\right) \boldsymbol{\lambda}^{\boldsymbol{j}}\left(\boldsymbol{\lambda}^{\prime}\right)^{\ell},
\end{aligned}
$$


from which it is clear that $\varphi\|X\| X^{\prime}=\varphi\left\|X^{\prime}\right\| X$. Moreover, by using the elliptic transformation, we find it equals

$$
\begin{aligned}
& \rho(X) \rho\left(X^{\prime}\right) \sum_{\boldsymbol{j}, \ell, \boldsymbol{m}}\left(\varphi_{0, \boldsymbol{j}+\boldsymbol{\ell}+\boldsymbol{m}} \mid X\right)\left(\begin{array}{c}
\boldsymbol{j}+\boldsymbol{\ell}+\boldsymbol{m} \\
\boldsymbol{j}, \boldsymbol{\ell}, \boldsymbol{m}
\end{array}\right) \boldsymbol{\lambda}^{\boldsymbol{j}}\left(\boldsymbol{\lambda}^{\prime}\right)^{\ell}\left(-\boldsymbol{\lambda}^{\prime}\right)^{\boldsymbol{m}}= \\
& \rho(X) \rho\left(X^{\prime}\right) \sum_{\boldsymbol{j}}\left(\varphi_{0, \boldsymbol{j}} \mid X\right) \boldsymbol{\lambda}^{\boldsymbol{j}},
\end{aligned}
$$

where the right-hand equals $\rho\left(X^{\prime}\right) \varphi \| X$. by definition.

Next, one has

$$
\begin{aligned}
\left(\varphi \| X+X^{\prime}\right) & =\rho\left(X+X^{\prime}\right) \sum_{\ell}\left(\varphi_{0, \ell} \mid X+X^{\prime}\right)\left(\boldsymbol{\lambda}+\boldsymbol{\lambda}^{\prime}\right)^{\ell} \\
& =\rho(X) \rho\left(X^{\prime}\right) \zeta_{X, X^{\prime}} \sum_{\ell}\left(\varphi_{0, \ell}|X| X^{\prime}\right)\left(\boldsymbol{\lambda}+\boldsymbol{\lambda}^{\prime}\right)^{\ell} \\
& =\rho(X) \rho\left(X^{\prime}\right) \zeta_{X, X^{\prime}} \sum_{\boldsymbol{j}, \ell}\left(\varphi_{0, \boldsymbol{j}+\ell} \mid X\right)\left(\begin{array}{c}
\boldsymbol{j}+\boldsymbol{\ell} \\
\boldsymbol{j}
\end{array}\right)\left(-\boldsymbol{\lambda}^{\prime}\right)^{\boldsymbol{j}}\left(\boldsymbol{\lambda}+\boldsymbol{\lambda}^{\prime}\right)^{\ell} \\
& =\rho(X) \rho\left(X^{\prime}\right) \zeta_{X, X^{\prime}} \sum_{\boldsymbol{j}}\left(\varphi_{0, \boldsymbol{j}} \mid X\right) \boldsymbol{\lambda}^{\boldsymbol{j}} \\
& =\rho\left(X^{\prime}\right) \zeta_{X, X^{\prime}} \varphi \| X .
\end{aligned}
$$

Finally, the fact that $\varphi \| X$ is a quasi-Jacobi form follows directly from the definition of $\Gamma_{X}$ and the previous properties.

\subsection{Taylor coefficients}

Let $X=\left(\begin{array}{l}\boldsymbol{\lambda} \\ \boldsymbol{\mu}\end{array}\right) \in M_{2, n}(\mathbb{Q}), M \in M_{n}(\mathbb{Q})$ and $\varphi \in \operatorname{Mer}_{n}$. We now study the Taylor coefficients of $\varphi \|_{M} X$ around $z=0$. In case $\varphi$ is a strictly meromorphic quasiJacobi form, recall that all poles $\boldsymbol{z}$ lie on a hyperplane of the form $\boldsymbol{s} \cdot \boldsymbol{z} \in u \tau+v+L_{\tau}$ for some $s \in \mathbb{Z}^{n}$ and $u, v \in \mathbb{Q} / \mathbb{Z}$ by Theorem 5.4.2(ii). From now on we assume that $s=e_{i}$ for some $i$, so that a Laurent series of $\varphi \| X$ of the form

$$
\sum_{\ell_{1} \geq L} \cdots \sum_{\ell_{n} \geq L} a_{\ell_{1}, \ldots, \ell_{n}}\left(z_{1}-\lambda_{1} \tau-\mu_{1}\right)^{\ell_{1}} \cdots\left(z_{n}-\lambda_{n} \tau-\mu_{n}\right)^{\ell_{n}}
$$

for some $L \in \mathbb{Z}$ and $a_{\ell} \in \mathbb{C}$ exists. For example, the poles of all the meromorphic quasi-Jacobi forms we encounter in the applications lie on the coordinate axes. 
Definition 5.9.1. We call the poles of a meromorphic function $\varphi: \mathfrak{h} \times \mathbb{C}^{n} \rightarrow \mathbb{C}$ orthogonal if the set of poles of $\varphi(\tau, \cdot)$ is given by a union of special hyperplanes of the form

$$
z_{s} \in u \tau+v+L_{\tau}
$$

for some $s \in\{1, \ldots, n\}$ and $u, v \in \mathbb{Q} / \mathbb{Z}$.

Now that we introduced orthogonal poles, above, and the double slash action in Definition 5.8.1, we defined the "Taylor coefficients" of a family of functions in the following way. Recall that in case $\varphi$ is a Jacobi form, there is a canonical choice for the family of functions $\varphi$ which is part of the data of these "Taylor coefficients" (see Convention 5.8.2).

Definition 5.9.2. Let $M \in M_{n}(\mathbb{Q})$ and $\varphi=\left\{\varphi_{i, j}\right\}$, where $\varphi_{i, j}: \mathfrak{h} \times \mathbb{C}^{n} \rightarrow \mathbb{C}$, be a family of meromorphic functions indexed by a finite subset of $\mathbb{Z}_{\geq 0} \times \mathbb{Z}_{\geq 0}^{n}$, with $\varphi:=\varphi_{0,0} \in \operatorname{Mer}_{n}^{M}$ such that all poles of $\varphi$ are orthogonal. Let $g_{\ell}(\varphi)$ as the $\ell$ th Laurent coefficient of $\varphi$ :

$$
(\varphi)(\tau, \boldsymbol{z})=\sum_{\ell} g_{\ell}(\varphi)(\tau) \boldsymbol{z}^{\ell}
$$

For all $X \in M_{2, n}(\mathbb{R})$, we define the "Taylor coefficient" $g_{\ell}^{X}(\varphi)$ as $g_{\ell}\left(\varphi \|_{M} X\right)$. Also, denote

$$
g_{\boldsymbol{\ell}, s}^{X}(\boldsymbol{\varphi})=g_{\boldsymbol{\ell}}\left(\sum_{i+|\boldsymbol{j}|=s}\left(\varphi_{i, \boldsymbol{j}} \| X\right)(\boldsymbol{z}) \boldsymbol{z}^{\boldsymbol{j}}\right) \quad\left(s \in \mathbb{Z}_{\geq 0}\right) .
$$

Remark 5.9.3. One may be tempted to write

$$
\text { " } g_{\ell, s}^{X}(\varphi)=\sum_{i+|j|=s} g_{\ell-j}\left(\varphi_{i, j} \| X\right) " .
$$

However, we do not assume that the functions $\varphi_{i, j}$ admit orthogonal poles, so the Taylor expansion of $\varphi_{i, j}$ may not exist. For example, taking $\varphi=F_{2}$ (defined by Definition 1.4.2), we will see later that $\varphi_{0, e_{1}}\left(z_{1}, z_{2}\right)=\frac{1}{\Theta\left(z_{1}+z_{2}\right)}$, which has a pole whenever $z_{1}+z_{2}=0$. Theorem 5.9.9 implicitly shows that the notation $g_{\ell, s}^{X}(\boldsymbol{\varphi})$ is well-defined for a quasi-Jacobi form $\varphi$ with all poles orthogonal.

The data $\left\{g_{\ell}^{X}(\varphi)\right\}$ uniquely determines $\varphi$ as well as the family $\varphi=\left\{\varphi_{i, j}\right\}$. Hence, it is natural to ask under which conditions on $g_{\ell}^{X}(\varphi)$ the function $\varphi$ is a meromorphic quasi-Jacobi form. Before we answer this question, we study the modular properties of $g_{\ell}^{X}(\varphi)$ given $\varphi$ is a meromorphic quasi-Jacobi form. As a corollary of the previous proposition on $\varphi \| X$, generalizing [EZ85, Theorem 1.3] to quasi-Jacobi forms, we show that $g_{0}^{X}(\varphi)$ is a quasimodular form. 
Corollary 5.9.4. Let $\varphi$ be a holomorphic quasi-Jacobi form of weight $k$ and index $M$. For all $X \in M_{2, n}(\mathbb{Q})$, the function $g_{0}^{X}(\varphi)$ is a holomorphic quasimodular form of weight $k$ for the group $\Gamma_{X}$ (defined by (5.17)). Moreover,

$$
\mathfrak{d}_{\tau} g_{\mathbf{0}}^{X}(\boldsymbol{\varphi})=g_{\mathbf{0}}^{X}\left(\mathfrak{d}_{\tau} \boldsymbol{\varphi}\right)
$$

Proof. Let $X \in M_{2, n}(\mathbb{Q})$ and $\gamma \in \Gamma_{X}$. Then, by Proposition 5.8.3(iii) one finds

$$
\left(g_{\mathbf{0}}^{X}(\boldsymbol{\varphi}) \mid \gamma\right)(\tau)=(\varphi \| X \mid \gamma)(\tau, \mathbf{0})=\sum_{i} g_{\mathbf{0}}^{X}\left(\boldsymbol{\varphi}_{i}\right)\left(\frac{c}{c \tau+d}\right)^{i}
$$

where $\varphi_{i}$ denotes the family corresponding to $\varphi_{i, \mathbf{0}}$. Hence, $g_{\mathbf{0}}^{X}(\varphi)$ is a quasimodular form for this group and $\mathfrak{d}_{\tau}^{r} g_{0}^{X}(\varphi)=\frac{g_{0}^{X}\left(\varphi_{r}\right.}{r !}=g_{0}^{X}\left(\mathfrak{d}_{\tau}^{r} \varphi\right)$. Holomorphicity in $\mathfrak{h}$ and at infinity follows directly as $\varphi$ is a holomorphic Jacobi form.

The quasimodularity of the other coefficients $g_{\ell}^{X}(\varphi)$ can be understood in terms of lower coefficients in two ways. First of all, certain linear combinations of derivatives of these coefficients are modular. Secondly the action of $\mathfrak{d}_{\tau}^{i}$ on $g_{\ell}^{X}(\boldsymbol{\varphi})$ can be expressed in terms of other coefficients.

We first show that these two ways are equivalent. Denote by $(x)_{n}$ the Pochhammer symbol $(x)_{n}=x(x+1) \cdots(x+n-1)$.

Proposition 5.9.5. Let $g=g_{k}, g_{k-2} \ldots, g_{k-2 p}$ be quasimodular forms of depth at most $p$ and weight $k, k-2 \ldots, k-2 p$ respectively. Then the following are equivalent:

(i) $\mathfrak{d}_{\tau}^{i} g=g_{k-2 i}$ for $i=0, \ldots, p$;

(ii) The functions

$$
\begin{cases}\sum_{0 \leq m \leq p-i}(-1)^{m} \frac{D^{m} g_{k-2 i-2 m}}{(k-2 i-m-1)_{m} m !} & \text { if } k-2 p>0 \text { or } i<p-1 \\ g_{2}-\mathbb{e}_{2} g_{0} & \text { if } k-2 p=0 \text { and } i=p-1\end{cases}
$$

for $i=0, \ldots, p-1$ are modular forms of weight $k-2 i$;

(iii) The functions

$$
\sum_{0 \leq m \leq p-i}(-1)^{m} \frac{\left(D+\mathbb{e}_{2}\right)^{m} g_{k-2 i-2 m}}{\left(k-2 i-m-\frac{3}{2}\right)_{m} m !}
$$

for $i=0, \ldots, p-1$ are modular forms of weight $k-2 i$. 
Remark 5.9.6. Observe that as $m \leq p-i$ and $i \leq p-1$ one has that

$$
k-2 i-m-1 \geq k-2 p .
$$

Hence, the numerator $(k-2 i-m-1)_{m}$ vanishes in case $k-2 p=0$ and $i=$ $p-1$ for $m-1$. In this case $g_{k-2 i-2 m}$ is a modular form of weight 0 , hence a constant function. Therefore, also the numerator $D^{m} g_{k-2 i-2 m}$ vanishes in this case. One can think of $\mathbb{e}_{2}$ as being the appropriate regularisation of this ill-defined ratio. Alternatively, in the third equivalence, one can replace $D$ by $D+\mathbb{e}_{2}$ in which case the numerator never vanishes. Finally, if one would replace $D$ by $\mathbb{e}_{2}$, one would obtain a generalisation of the functions $\varphi_{n}$ of [Bri18, Proposition 3.1].

Proof. Assume that $\mathfrak{d}_{\tau}^{i} g=g_{i}$. As $g$ is of depth at most $p$, this implies that $\mathfrak{d}_{\tau} g_{i}=$ $g_{i-2}$ for all $i$, where $g_{i}$ is taken to be zero when it is not defined. Hence, using $\left[\mathfrak{d}_{\tau}, D_{\tau}\right]=W$ (see Remark 5.7.3), it follows that applying $\mathfrak{d}_{\tau}$ to a term in the sum in (ii) yields

$$
(-1)^{m} \frac{D_{\tau}^{m} g_{k-2 i-2 m-2}}{(k-2 i-m-1)_{m} m !}-(-1)^{m-1} \frac{D_{\tau}^{m-1} g_{k-2 i-2 m}}{(k-2 i-(m-1)-1)_{m-1}(m-1) !},
$$

where the second term is taken to be zero when $m=0$. Also the first term vanishes when $m=p-i$ as $f_{k-2 p-2}$ is set to be zero. Hence, after applying $\mathfrak{d}_{\tau}$ the sum becomes a telescoping sum, equal to zero. Also $\mathfrak{d}_{\tau}\left(g_{2}-\mathbb{e}_{2} g_{0}\right)=g_{0}-g_{0}=0$. The third statements follows by the same argument, mutatis mutandis.

The converse statement follows inductively by using that all but two terms in the same sum equal to zero cancel, hence these terms are equal.

In order to express the derivatives with respect to $\mathfrak{d}_{\tau}$ of the coefficients of $\varphi$, we introduce the following notation describing how the index mixes the coefficients.

Definition 5.9.7. Given $M \in M_{n}(\mathbb{Q}), \varphi \in \operatorname{Mer}_{n}^{M}$ and a family $\varphi$ as before, for $r, s \in \mathbb{Z}_{\geq 0}, \boldsymbol{\ell} \in \mathbb{Z}^{n}$ and $X \in M_{2, n}(\mathbb{Q})$, we write $\mathcal{Q}^{r} g_{\ell, s}^{X}(\varphi)$ for

$$
\mathcal{Q}^{r} g_{\ell, s}^{X}(\boldsymbol{\varphi})=g_{\ell}\left(Q_{M}^{r}(\boldsymbol{z}) \sum_{i+|\boldsymbol{j}|=s}\left(\varphi_{i, j} \| X\right)(\boldsymbol{z}) \boldsymbol{z}^{j}\right),
$$

where the Laurent coefficients $g_{\ell}$ are given by Definition 5.9.2. Moreover, the functions $\xi_{\ell}^{X}(\varphi)(\tau)$ are defined by (again $\ell \in \mathbb{Z}^{n}, X \in M_{2, n}(\mathbb{Q})$ )

$$
\xi_{\ell}^{X}(\boldsymbol{\varphi}):=\left\{\begin{array}{l}
\sum_{r} \sum_{s \leq r}(-1)^{r} \frac{D_{\tau}^{r}\left(\mathcal{Q}^{r-s} g_{\ell, s}^{X}\right)(\boldsymbol{\varphi})}{(k+|\ell|-s-1)_{r}(r-s) !} \\
g_{\ell}^{X}(\boldsymbol{\varphi})-\mathbb{E}_{2}\left(\left(\mathcal{Q} g_{\ell}^{X}\right)(\boldsymbol{\varphi})+\left(g_{\ell, 1}^{X}\right)(\boldsymbol{\varphi})\right)
\end{array} \quad k=0,|\ell|=2,\right.
$$

where $(x)_{n}=x(x+1) \cdots(x+n-1)$ denotes the Pochhammer symbol. Abbreviate $\xi_{\ell}^{X}(\varphi)$ by $\xi_{\ell}(\varphi)$ if $X$ is the zero matrix. 
Remark 5.9.8. We formulate all results below for $\xi_{\ell}^{X}(\varphi)$ as defined above, but by Proposition 5.9.5 all results remain valid after replacing $\xi_{\ell}^{X}(\varphi)$ by

$$
\sum_{r}(-1)^{r} \sum_{s} \frac{\left(D_{\tau}+\mathbb{e}_{2}\right)^{r}\left(\mathcal{Q}^{r-s} g_{\ell, s}^{X}\right)(\varphi)}{\left(k+|\ell|-r-\frac{3}{2}\right)_{r}(r-s) !} .
$$

Note that this equation, as well as Equation 5.19, can be inverted, expressing $\xi_{\ell}^{X}(\varphi)$ as linear combination of derivatives of certain $g_{m}^{X}(\varphi)$.

This allows to characterise invariance under the modular action by its Taylor coefficients, generalizing [EZ85, Theorem 3.2]:

Theorem 5.9.9. Let $\Gamma$ be a congruence subgroup, $k \in \mathbb{Z}$ and $M \in M_{n}(\mathbb{Q})$. Let $\varphi=\left\{\varphi_{i, j}\right\}$ as before a family of meromorphic functions $\mathfrak{h} \times \mathbb{C}^{n} \rightarrow \mathbb{C}$ with $\varphi:=$ $\varphi_{0,0} \in \operatorname{Mer}_{n}^{M}$ and admitting a Laurent expansion around $\boldsymbol{z}=\mathbf{0}$. Then the following are equivalent:

(i) The function $\varphi$ satisfies the quasimodular transformation (5.13)

$$
\left(\left.\varphi\right|_{k, Q} \gamma\right)(\tau, z)=\sum_{i, \boldsymbol{j}} \varphi_{i, \boldsymbol{j}}(\tau, \boldsymbol{z})\left(\frac{c}{c \tau+d}\right)^{i+|\boldsymbol{j}|} \boldsymbol{z}^{\boldsymbol{j}}
$$

for all $\gamma \in \Gamma$;

(ii) The coefficients $g_{\ell}(\varphi)$ are quasimodular forms of weight $k+|\ell|$ uniquely determined by the coefficients of the $\varphi_{i, j}$, i.e.,

$$
\mathfrak{d}_{\tau}^{r} g_{\ell}(\varphi)=\sum_{s} \frac{r !}{(r-s) !} \mathcal{Q}^{r-s} g_{\ell, s}(\varphi)
$$

(iii) The functions $\xi_{\ell}(\varphi)$ are modular forms of weight $k+|\ell|$ on $\Gamma$ for all $\ell$.

Proof. Expanding (5.13) yields

$$
\sum_{\ell} \frac{g_{\ell}(\varphi)(\gamma \tau)}{(c \tau+d)^{k+2 m+s}} \boldsymbol{z}^{\ell}=\sum_{\ell} \sum_{r, s} \frac{g_{\ell, s}(\varphi)(\tau)}{r !}\left(\frac{c}{c \tau+d}\right)^{r+s} Q_{M}(\boldsymbol{z})^{r} \boldsymbol{z}^{\ell}
$$

Extracting on both sides the coefficient of $z^{\ell}$ yields

$$
\mathfrak{d}_{\tau}^{r} g_{\ell}(\varphi)=\sum_{s} \frac{r !}{(r-d) !} \mathcal{Q}^{r-s} g_{\ell, s}(\varphi) .
$$

Also, the coefficient $g_{\ell}(\varphi)$ is holomorphic in $\mathfrak{h}$ as well as at the cusps of $\Gamma$, because of the analytic properties of the functions $\varphi_{i, j}$. The rest of the statement follows from Proposition 5.9.5. 
Using this result, one can characterise a quasi-Jacobi form $\varphi$ by its Taylor coefficients in three ways, i.e., by considering $g_{\ell}^{X}(\varphi)$ as a vector-valued quasimodular form, by the modularity of the functions $\xi_{\ell}^{X}(\varphi)$, and finally by the action of $\mathfrak{d}_{\tau}$ on the quasimodular form $g_{\ell}^{X}(\varphi)$. Write $f^{X}$ for $g_{\ell}^{X}(\varphi)$ or $\xi_{\ell}^{X}(\varphi)$. Then, the 'elliptic transformation' of the quasimodular form $f^{X}$ is given by

$$
f^{X+X^{\prime}}=\rho\left(X^{\prime}\right) \zeta_{X, X^{\prime}} f^{X} \quad \text { for all } X^{\prime} \in M_{2, n}(\mathbb{Z})
$$

Recall that $g_{\ell}^{X}(\varphi)$ and $\xi_{\ell}^{X}(\varphi)$ are only defined when the zeros of $\varphi$ are orthogonal (see Definition 5.9.1) and depend on a family of functions $\varphi_{i, j}: \mathfrak{h} \times \mathbb{C}^{n} \rightarrow \mathbb{C}$. In case $\varphi$ is a quasi-Jacobi form this family $\varphi=\left\{\varphi_{i, j}\right\}$ determines the transformation of $\varphi$; without the assumption that $\varphi$ is as a quasi-Jacobi form we have the following statement refining Theorem E.

Theorem 5.9.10 ( = Theorem E). Let $k \in \mathbb{Z}, M \in M_{n}(\mathbb{Q})$ and $\varphi \in \operatorname{Mer}_{n}^{M}$ such that the set of poles of $\varphi$ is orthogonal. Given a family $\varphi=\left\{\varphi_{i, j}\right\}$, indexed by $(i, \boldsymbol{j})$ in a finite subset of $\mathbb{Z}_{\geq 0} \times \mathbb{Z}_{\geq 0}^{n}$, of meromorphic functions $\varphi_{i, j}: \mathfrak{h} \times \mathbb{C}^{n} \rightarrow \mathbb{C}$ with $\varphi=\varphi_{0,0}$, the following are equivalent:

(i) The function $\varphi$ is a meromorphic quasi-Jacobi form of weight $k$ and index $M$ for which the functions $\varphi_{i, j}$ determine its transformation behaviour as in (5.13) and (5.14).

(ii) For all $X=\left(\begin{array}{c}\boldsymbol{\lambda} \\ \boldsymbol{\mu}\end{array}\right) \in M_{2, n}(\mathbb{Q})$ with $\boldsymbol{\lambda} \tau+\boldsymbol{\mu}$ not a pole of $\varphi$, the function $g_{\mathbf{0}}^{X}(\boldsymbol{\varphi})$ is a vector valued quasimodular form satisfying (5.20) and transforming as

$$
\left.g_{\mathbf{0}}^{X}(\varphi)\right|_{k} \gamma=\sum_{s} g_{\mathbf{0}, s}^{\gamma X}(\varphi)\left(\frac{c}{c \tau+d}\right)^{s}
$$

(ii) $^{\prime}$ For all $X \in M_{2, n}(\mathbb{Q})$ the function $g_{\boldsymbol{\ell}}^{X}(\varphi)$ is a vector valued quasimodular form satisfying (5.20) and transforming as

$$
\left.g_{\ell}^{X}(\varphi)\right|_{k} \gamma=\sum_{r} \sum_{s} \frac{1}{(r-s) !} \mathcal{Q}^{r-s} g_{\ell, s}^{\gamma X}(\varphi)\left(\frac{c}{c \tau+d}\right)^{r} .
$$

(iii) For all $\ell \in \mathbb{Z}^{n}$ the functions $\xi_{\ell}(\varphi)$ are modular forms of weight $k+|\ell|$ for $\mathrm{SL}_{2}(\mathbb{Z})$ and for all $X \in M_{2, n}(\mathbb{Q})$ the functions $\xi_{0}^{X}(\varphi)$ satisfy (5.20).

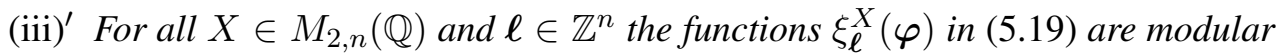
forms of weight $k+|\ell|$ for $\Gamma_{X}$ and satisfy (5.20). 
(iv) For all $X \in M_{2, n}(\mathbb{Q})$ and $\ell \in \mathbb{Z}^{n}$ the functions $g_{\ell}^{X}(\varphi)$ are quasimodular forms of weight $k+|\ell|$ for $\Gamma_{X}$, satisfying (5.20) and

$$
\mathfrak{d}_{\tau}^{r} g_{\ell}^{X}(\boldsymbol{\varphi})=\sum_{s} \frac{r !}{(r-s) !}\left(\mathcal{Q}^{r-s} g_{\ell, s}^{X}\right)(\boldsymbol{\varphi}) .
$$

Proof. (i) implies (iv): Let $X \in M_{2, n}(\mathbb{Q})$ and $\gamma \in \Gamma_{X}$ be given. By Proposition 5.8.3 the function $\varphi \| X$ satisfies the conditions of Theorem 5.9.9 for $\Gamma=\Gamma_{X}$. Moreover, by the same proposition the coefficients only depend on $X \bmod M_{2, n}(\mathbb{Z})$ up to the factor $\rho\left(X^{\prime}\right) \zeta_{X, X^{\prime}}$.

(iv) implies (iii)': This follows directly from Theorem 5.9 .9 for $\Gamma=\Gamma_{X}$.

(iii)' implies (iii): Observe that $\Gamma_{X}=\mathrm{SL}_{2}(\mathbb{Z})$ for $X$ equal to the zero matrix. Hence, we simply forget some of the properties of $\xi_{\ell}^{X}$.

(iii) implies (ii)': As $\xi_{\ell}$ is a modular form for $\mathrm{SL}_{2}(\mathbb{Z})$ for all $\ell \in \mathbb{Z}^{n}$, it follows by Theorem 5.9.9 that $\varphi$ satisfies the quasimodular transformation for all $\gamma \in \mathrm{SL}_{2}(\mathbb{Z})$. As by Proposition 5.8.3

$$
\varphi \|\left. X\right|_{k} \gamma=\sum_{i, \boldsymbol{j}}\left(\varphi_{i, \boldsymbol{j}} \| \gamma X\right)\left(\frac{c}{c \tau+d}\right)^{i}\left(\frac{c \boldsymbol{z}}{c \tau+d}\right)^{j},
$$

the result follows by extracting the coefficients of $z$ on both sides. Finally, note that $\xi_{0}^{X}=g_{0}^{X}$.

(ii) ${ }^{\prime}$ implies (ii): This follows directly by restricting to $\ell=\mathbf{0}$.

(ii) implies (i): Suppose $\boldsymbol{z}=\boldsymbol{\lambda} \tau+\boldsymbol{\mu}$ with $X=\left(\begin{array}{c}\boldsymbol{\lambda} \\ \boldsymbol{\mu}\end{array}\right) \in M_{2, n}(\mathbb{Q})$ not being a pole of $\varphi$. Let $\varphi_{i}$ be the family of functions corresponding to $\varphi_{i, \mathbf{0}}$. Using (ii), for $\gamma \in \mathrm{SL}_{2}(\mathbb{Z})$ one has that $\left.\left(g_{\mathbf{0}}^{X}(\varphi)\right)\right|_{k} \gamma$ equals

$$
\begin{aligned}
& \sum_{i} g_{\mathbf{0}}^{\gamma X}\left(\boldsymbol{\varphi}_{i}\right)(\tau)\left(\frac{c}{c \tau+d}\right)^{i} \\
= & \rho(-\gamma X) \sum_{i, \boldsymbol{\ell}}\left(\varphi_{i, \ell} \mid \gamma X\right)(\tau, 0)\left(\frac{c}{c \tau+d}\right)^{i}\left(\boldsymbol{\lambda}^{\gamma}\right)^{\ell} \\
= & \rho(-\gamma X) \sum_{i, \boldsymbol{j}, \boldsymbol{\ell}}\left(\varphi_{i, \boldsymbol{j}+\boldsymbol{\ell}} \mid \gamma X\right)(\tau, 0)\left(\frac{c}{c \tau+d}\right)^{i}\left(\begin{array}{c}
\boldsymbol{j}+\boldsymbol{\ell} \\
\boldsymbol{j}
\end{array}\right)\left(\frac{c\left(\boldsymbol{\lambda}^{\gamma} \tau+\boldsymbol{\mu}^{\gamma}\right)}{c \tau+d}\right)^{j} \lambda^{\ell} .
\end{aligned}
$$

On the other hand,

$$
\begin{aligned}
\left.\left(g_{\mathbf{0}}^{X}(\boldsymbol{\varphi})\right)\right|_{k} \gamma & =\rho(-X) \sum_{\ell}\left(\varphi_{0, \ell}|X|_{k} \gamma\right)(\tau, 0) \lambda^{\ell} \\
& =\rho(-\gamma X) \sum_{\ell}\left(\left.\varphi_{0, \ell}\right|_{k} \gamma \mid \gamma X\right)(\tau, 0) \lambda^{\ell} .
\end{aligned}
$$


Combining the identities yields

$$
\begin{aligned}
& \sum_{\ell}\left(\left.\varphi_{0, \ell}\right|_{k} \gamma \mid \gamma X\right)(\tau, 0) \lambda^{\ell}= \\
& \sum_{i, \boldsymbol{j}, \boldsymbol{\ell}}\left(\varphi_{i, \boldsymbol{j}+\ell} \mid \gamma X\right)(\tau, 0)\left(\frac{c}{c \tau+d}\right)^{i}\left(\begin{array}{c}
\boldsymbol{j}+\boldsymbol{\ell} \\
\boldsymbol{j}
\end{array}\right)\left(\frac{c\left(\boldsymbol{\lambda}^{\gamma} \tau+\boldsymbol{\mu}^{\gamma}\right)}{c \tau+d}\right)^{j} \boldsymbol{\lambda}^{\ell}
\end{aligned}
$$

As both sides equal $\rho(\gamma X) g_{0}^{X}(\varphi)$, which is periodic with finite period as a function of $\boldsymbol{\lambda}$, the constant terms with respect to $\boldsymbol{\lambda}$ agree. Hence,

$$
(\varphi|\gamma| X)(\tau, 0)=\sum_{i, \boldsymbol{j}}\left(\varphi_{i, \boldsymbol{j}} \mid X\right)(\tau, 0)\left(\frac{c}{c \tau+d}\right)^{i}\left(\frac{c(\boldsymbol{\lambda} \tau+\boldsymbol{\mu})}{c \tau+d}\right)^{j}
$$

for all $X=\left(\begin{array}{l}\boldsymbol{\lambda} \\ \boldsymbol{\mu}\end{array}\right) \in M_{2, n}(\mathbb{Q})$ with $X$ not corresponding to a pole. Therefore, $\varphi$ satisfies the quasimodular transformation for all $\boldsymbol{z}$ of the given form. As the set $\left(\mathbb{Q}^{n} \tau \times \mathbb{Q}\right)^{n} \backslash P_{\varphi}$, with $P_{\varphi}$ the set of poles of $\varphi$, lies dense in $\mathbb{C}^{n}$ for all $\tau \in \mathfrak{h}$, the function $\varphi$ satisfies the quasimodular transformation equation.

For the elliptic transformation, we again assume $\boldsymbol{z}=\boldsymbol{\lambda} \tau+\boldsymbol{\mu}$ with $X=\left(\begin{array}{l}\boldsymbol{\lambda} \\ \boldsymbol{\mu}\end{array}\right) \in$ $M_{2, n}(\mathbb{Q})$ not being a pole. Given $X^{\prime}=\left(\boldsymbol{\lambda}^{\prime}, \boldsymbol{\mu}^{\prime}\right) \in M_{2, n}(\mathbb{Z})$, we have

$$
\begin{aligned}
\sum_{\ell}\left(\varphi_{0, \ell} \mid X\right)(\tau, 0) \lambda^{\ell} & =\rho(X) g_{\mathbf{0}}^{X}(\boldsymbol{\varphi}) \\
& =\rho(X) \rho\left(-X^{\prime}\right) \zeta_{X+X^{\prime}}^{-1} g_{\mathbf{0}}^{X+X^{\prime}}(\boldsymbol{\varphi}) \\
& =\rho\left(-X^{\prime}\right) \zeta_{X+X^{\prime}}^{-1} \sum_{\ell}\left(\varphi_{0, \ell}\left|X^{\prime}\right| X\right)(\tau, 0)\left(\boldsymbol{\lambda}+\boldsymbol{\lambda}^{\prime}\right)^{\ell}
\end{aligned}
$$

The coefficients of $\boldsymbol{\lambda}$ agree, so

$$
\left(\varphi \mid X^{\prime}\right)(\tau, \boldsymbol{z})=\rho\left(X^{\prime}\right) \zeta_{X+X^{\prime}} \sum_{j} \varphi_{0, \boldsymbol{j}}(\tau, z)\left(-\boldsymbol{\lambda}^{\prime}\right)^{j}
$$

for all $z$ of the given form. As before by continuity of $\varphi$ the above equation holds for all $z$.

Remark 5.9.11. The proof of the above result also applies to weak Jacobi forms, after replacing 'meromorphic Jacobi form' and '(quasi)modular' by 'weak Jacobi form' and 'weakly holomorphic (quasi)modular', respectively.

Specializing to holomorphic Jacobi forms (instead of meromorphic quasi-Jacobi forms), we obtain the following result, generalizing the main results on Taylor coefficients of Jacobi forms in [EZ85] to multivariable Jacobi forms. 
Corollary 5.9.12. Let $k \in \mathbb{Z}, M \in M_{n}(\mathbb{Q})$ and $\varphi=\{\varphi\}$ with $\varphi \in \operatorname{Hol}_{n}^{M}$. Then, the following are equivalent:

(i) The function $\varphi$ is a holomorphic Jacobi form of weight $k$ and index $M$.

(ii) For all $X \in M_{2, n}(\mathbb{Q})$ the function $g_{0}^{X}(\varphi)$ is a vector valued modular form satisfying (5.20) and transforming as

$$
\left.g_{0}^{X}(\varphi)\right|_{k} \gamma=g_{0}^{\gamma X}(\varphi) .
$$

(ii') For all $X \in M_{2, n}(\mathbb{Q})$ the function $g_{\ell}^{X}(\varphi)$ is a vector valued quasimodular form satisfying (5.20) and transforming as

$$
\left.g_{\ell}^{X}(\boldsymbol{\varphi})\right|_{k} \gamma=\sum_{r} \frac{1}{r !}\left(\mathcal{Q}^{r} g_{\ell}^{\gamma X}\right)(\boldsymbol{\varphi})\left(\frac{c}{c \tau+d}\right)^{r} .
$$

(iii') For all $X \in M_{2, n}(\mathbb{Q})$ and $\ell \in \mathbb{Z}^{n}$ the functions $\xi_{\ell}^{X}(\varphi)$ given by

$$
\xi_{\ell}^{X}(\boldsymbol{\varphi})=\sum_{r}(-1)^{r} \frac{D_{\tau}^{r}\left(\mathcal{Q}^{r} g_{\ell}^{X}\right)(\varphi)}{(k+|\ell|-1)_{r} r !}
$$

are modular forms of weight $k+|\ell|$ for $\mathrm{SL}_{2}(\mathbb{Z})$ and satisfy (5.20).

(iv) For all $X \in M_{2, n}(\mathbb{Q})$ and $\ell \in \mathbb{Z}^{n}$ the functions $g_{\ell}^{X}(\varphi)$ are quasimodular forms of weight $k+|\ell|$ for $\Gamma_{X}$, satisfying (5.20) and

$$
\mathfrak{d}_{\tau}^{r} g_{\ell}^{X}(\boldsymbol{\varphi})=\left(\mathcal{Q}^{r} g_{\ell}^{X}\right)(\boldsymbol{\varphi})
$$



CHAPTER 6

\section{The Bloch-Okounkov theorem for congruence subgroups}

\subsection{Introduction}

Given $a \in \mathbb{Q}, k \geq 1$ and $\lambda \in \mathscr{P}$, in (1.19) we introduced the functions $Q_{k}(\lambda, a)$ as generalisations of the shifted symmetric functions given by

$$
\beta_{k}(a)+\frac{1}{(k-1) !} \sum_{i=1}^{\infty}\left(\boldsymbol{e}(a)^{\lambda_{i}-i}\left(\lambda_{i}-i+\frac{1}{2}\right)^{k-1}-\boldsymbol{e}(a)^{-i}\left(-i+\frac{1}{2}\right)^{k-1}\right),
$$

where $\sum_{k \in \mathbb{Z}} \beta_{k}(a)(2 \pi \mathrm{i} z)^{k-1}=\frac{\boldsymbol{e}(z / 2)}{\boldsymbol{e}(z+a)-1}$ and $\boldsymbol{e}(x)=e^{2 \pi \mathrm{i} x}$. The main properties satisfied by these functions are a consequence of the fact that

$$
\sum_{k \geq 0} Q_{k}(\lambda, a)(2 \pi \mathrm{i} z)^{k-1}=e\left(-\frac{1}{2} a\right) W_{\lambda}(z+a)
$$

and that $F_{n}\left(z_{1}, \ldots, z_{n}\right)=\left\langle W\left(z_{1}\right) \cdots W\left(z_{n}\right)\right\rangle_{q}$ is a quasi-Jacobi form. Up to a constant, these functions $Q_{k}(a)$ have been considered before in [EO06] for $a=\frac{1}{2}$ and in [Eng17] for all $a \in \mathbb{Q}$. It was shown that a certain modified $q$-bracket of any polynomial in these functions, excluding the function $Q_{1}(a)$, is quasimodular for $\Gamma_{1}(N)$ for some $N$.

In this chapter we will not change the $q$-bracket, nor exclude any of the functions (6.1), and nevertheless prove the following result for the graded algebra $\Lambda^{*}(N)$, contained in $\mathbb{C}^{\mathscr{P}}$, given by

$$
\Lambda^{*}(N):=\mathbb{Q}\left[Q_{k}(\cdot, a) \mid k \geq 1, a \in\left\{0, \frac{1}{N}, \ldots, \frac{N-1}{N}\right\}\right],
$$

with the grading given by assigning weight $k$ to $Q_{k}(\cdot, a)$. Given $N \geq 1$, write $(2, N)$ for $\operatorname{gcd}(2, N)$. Denote by $w_{M}$ the Fricke operator $w_{M}=\left(\begin{array}{cc}0 & -1 \\ M & 0\end{array}\right)$.

This chapter, as well as the previous and next chapter, are based on [I21b]. 
Theorem 6.1.1 (= Theorem F). Let $k \in \mathbb{Z}, N \geq 1$ and $M=(2, N) N$. For $f \in \Lambda^{*}(N)$ of weight $k$, the q-bracket $\langle f\rangle_{q}$ is a quasimodular form of weight $k$ for $w_{M}^{-1} \Gamma(M) w_{M}$.

Remark 6.1.2. The occurrence of the group $w_{M}^{-1} \Gamma(M) w_{M}$ in the theorem can be interpreted in at least two ways. First of all, equivalently $f$ as in the theorem has the property that $\langle f\rangle_{q_{M}}$ is a quasimodular form of level $M$, where $q_{M}:=q^{1 / M}$. Secondly, as $\Gamma_{1}\left(M^{2}\right) \leq w_{M}^{-1} \Gamma(M) w_{M}$, it follows that $\langle f\rangle_{q}$ is a quasimodular form for $\Gamma_{1}\left(M^{2}\right)$.

Moreover, the definition of $M$ indicates that the behaviour of $Q_{k}(a)$ is different when the numerator of $a$ is divisible by 2 . We will see that this can also by explained by the $n$-point functions $F_{n}$ : they are quasi-Jacobi forms of half-integral index.

The following theorem gives a refinement of Theorem 6.1.1 giving us $q$-brackets (or quotients of $q$-brackets) that are on $\Gamma_{1}(N)$ rather than only on the much smaller group $w_{M}^{-1} \Gamma(M) w_{M}$.

Theorem 6.1.3. Let $N \geq 1$. Given $k_{i} \in \mathbb{Z}, a_{i} \in \frac{1}{N} \mathbb{Z}$ for $i=1, \ldots, n$, denote $a=a_{1}+\ldots+a_{n}$ and $Q_{\boldsymbol{k}}(\cdot, \boldsymbol{a})=Q_{k_{1}}\left(\cdot, a_{1}\right) \cdots Q_{k_{n}}\left(\cdot, a_{n}\right)$. Then,

- If a $\in \mathbb{Z}$, then $\left\langle Q_{\boldsymbol{k}}(\cdot, \boldsymbol{a})\right\rangle_{q}$ is a quasimodular form for $\Gamma_{1}(N)$;

- If a $\notin \mathbb{Z}$, then $\frac{\left\langle Q_{\boldsymbol{k}}(\cdot, \boldsymbol{a})\right\rangle_{q}}{\left\langle Q_{1}(\cdot, a)\right\rangle_{q}}$ is a quasimodular form for $\Gamma_{1}(N)$.

Remark 6.1.4. Let $a \in \mathbb{Q}$. The function $\left\langle Q_{1}(\cdot, a)\right\rangle_{q}^{-1}$, equal to $\Theta(a)$, is a so-called Klein form; see, e.g., [KL81]. In particular, $\left\langle Q_{1}(\cdot, a)\right\rangle_{q}$ is ill-defined for $a \in \mathbb{Z} . \quad \triangle$

Theorem 6.1.1 should be compared with the results in [GJT16], where the authors consider certain functions $Q_{k}^{(p)}$ (see (6.6), where we define $Q_{k}^{(m)}$ also for composite $m$ ) in the context of studying $p$-adic analogues of the shifted symmetric functions (1.10). They show that the $q$-bracket of these functions is quasimodular for $\Gamma_{0}\left(p^{2}\right)$ and suggest that it is likely that products of these functions also have quasimodular $q$-brackets for the same group. We will see that the functions $Q_{k}^{(m)}$, as well as their products, are in fact elements of $\Lambda^{*}(m)$. Therefore, for all odd $m$ Theorem 6.1.1 implies that $q$-brackets of products of these functions are quasimodular for $\Gamma_{1}\left(m^{2}\right)$. That these $q$-brackets are indeed quasimodular for the bigger group $\Gamma_{0}\left(m^{2}\right)$ is the content of the next theorem. Write $\Lambda^{(N)}$ for the $\mathbb{Q}$-graded algebra generated by the functions $Q_{k}^{(m)}$ for all $m \mid N$.

Theorem 6.1.5. Let $N \geq 1$ and $k \in \mathbb{Z}$. For all homogeneous $f \in \Lambda^{(N)}$ of weight $k$ the function $\langle f\rangle_{q}$ is a quasimodular form of weight $k$ for $\Gamma_{0}\left(N^{2}\right)$. 
It should be noted that the above theorems hold true in a greater generality. For example, the so-called hook-length moments introduced in [CMZ18] and studied in the context of harmonic Maass forms for a congruence subgroup in [BOW20], also have natural generalisations obtained by studying their corresponding $n$-point functions. Similarly, the moment functions and their generalisations in Chapter 4 can equally well be generalised to congruence subgroups. Therefore, we will state and prove the above results in Section 6.2 in a more general setting that allows application to the hook-length moments and moment functions.

\subsection{General set-up}

We answer the question how to extend an algebra of functions on partitions for which the $q$-bracket is a quasimodular form for $\mathrm{SL}_{2}(\mathbb{Z})$ to one for a congruence subgroup $\Gamma$. More precisely, we consider quasimodular algebras for $\Gamma$ - a direct generalisation of Definition 4.1.1.

Definition 6.2.1. A quasimodular algebra for a congruence subgroup $\Gamma \leq \mathrm{SL}_{2}(\mathbb{Z})$ is a graded algebra of functions $f$ on the set of partitions for which $\langle f\rangle_{q}$ is a quasimodular form for $\Gamma$ of the same weight as $f$.

We now present a construction of a quasimodular algebra for a congruence subgroup given a quasimodular algebra for $\mathrm{SL}_{2}(\mathbb{Z})$. In order to do so, from now on we assume that $\Phi: \mathscr{P} \times \mathbb{C}^{r} \rightarrow \mathbb{C}$ is such that for all $n \geq 1$ there exists a weight $k \in \mathbb{Z}$ such that the function $\varphi_{n}^{\Phi}: \mathfrak{h} \times M_{n, r}(\mathbb{C}) \rightarrow \mathbb{C}$ given by

$$
\varphi_{n}^{\Phi}(\tau, Z):=\left\langle\prod_{i=1}^{n} \Phi\left(\cdot, Z_{i}\right)\right\rangle_{q},
$$

where $Z_{i}$ is the $i$ th row of $Z$, is a meromorphic quasi-Jacobi form of weight $k n$ which admits a Laurent expansion around all $Z \in M_{n, r}(\mathbb{Q})$ (after identifying $M_{n, r}(\mathbb{C}$ ) with $\left.\mathbb{C}^{n r}\right)$. Here, $\Phi(\lambda, \boldsymbol{z})$ can be thought of a generalisation of the generating series $W_{\lambda}(z)$ of the Bloch-Okounkov functions $Q_{k}$, defined by (1.13).

Definition 6.2.2. Given such a $\Phi$, for $\boldsymbol{a} \in \mathbb{Q}^{r}$, we denote by $f_{\boldsymbol{\ell}}^{\Phi}(\cdot, \boldsymbol{a})=f_{\boldsymbol{\ell}}(\cdot, \boldsymbol{a})$ : $\mathscr{P} \rightarrow \mathbb{C}$ the $\ell$ th Taylor coefficient of $\Phi(\boldsymbol{z})$ around $\boldsymbol{z}=\boldsymbol{a}$, i.e.,

$$
\Phi(\cdot, \boldsymbol{z})=\sum_{\ell} f_{\ell}(\cdot, \boldsymbol{a})(\boldsymbol{z}-\boldsymbol{a})^{\ell} .
$$

Define the graded $\mathbb{Q}$-algebra $\mathcal{F}^{\Phi}(N)=\mathcal{F}(N)$ as the algebra generated by the weight $k+|\ell|$ elements $f_{\boldsymbol{\ell}}(\cdot, \boldsymbol{a})$ for $\boldsymbol{a} \in \frac{1}{N} \mathbb{Z}^{r}, \boldsymbol{\ell} \in \mathbb{Z}^{r}$. 
Remark 6.2.3. By Theorem 5.9.10, up to a sign $f_{\boldsymbol{m}}(\cdot, \boldsymbol{a})$ and $f_{\boldsymbol{m}}(\cdot, \boldsymbol{b})$ agree whenever $\boldsymbol{a}-\boldsymbol{b} \in \mathbb{Z}^{r}$. Hence, in the definition one can assume that $\boldsymbol{a} \in[0,1)^{r}$.

For example, $Q_{\ell+1}(a)=e\left(-\frac{1}{2} a\right) f_{\ell}^{W}(a)($ see $(6.1))$ and $\Lambda^{*}(N)=\mathcal{F}^{W}(N)$.

Let $L \in M_{n, r}(\mathbb{Z})$ and $A \in M_{n, r}(\mathbb{Q})$. An arbitrary monomial $f_{L}$ in $\mathcal{F}^{\Phi}(N)$ is given by

$$
f_{L}(A):=f_{L_{1}}\left(A_{1}\right) \cdots f_{L_{n}}\left(A_{n}\right)
$$

with $L_{i}$ and $A_{i}$ the $i$ th row of $L$ and $A$, respectively. By construction of the Taylor coefficients of $\Phi$ as well as of $\varphi_{n}^{\Phi}$ we find

$$
\left\langle f_{L}^{\Phi}(A)\right\rangle_{q}=g_{L}^{(0, A)}\left(\varphi_{n}^{\Phi}\right),
$$

where on the right-hand side we identified $M_{n, r}(\mathbb{Z})$ and $M_{n, r}(\mathbb{Q})$ with $\mathbb{Z}^{n r}$ and $\mathbb{Q}^{n r}$, respectively, and as $\varphi_{n}^{\Phi}$ is a quasi-Jacobi form it uniquely determines the family of functions in the definition of the "Taylor coefficients"1.

The following result is the general statement of Theorem 6.1.1.

Theorem 6.2.4. Given $\Phi$ as above and $N \geq 1$, let $M=(2, N) N$. The algebra $\mathcal{F}^{\Phi}(N)$ is a quasimodular algebra for $w_{M}^{-1} \Gamma(M) w_{M}$.

Proof. Consider a monomial element $f_{L}(A)$ of $\mathcal{F}(N)$ as in (6.3), for some $L \in$ $M_{n, r}(\mathbb{Z})$ and $A \in M_{n, r}(\mathbb{Q})$. Write $X=(0, A)^{t}$. Then, $\left\langle f_{L}(A)\right\rangle_{q}=g_{L}^{X}\left(\varphi_{n}\right)$. This Taylor coefficient is quasimodular for $\Gamma_{X}$ by Theorem 5.9.10. Therefore, it suffices to show that the $q$-bracket respects the weight grading of $\mathcal{F}(N)$ and that $\Gamma_{X}$ contains $w_{N}^{-1} \Gamma(N) w_{N}$.

For the first, observe that the weight of $f$ is given by $\sum_{i=1}^{n}\left(k+\left|L_{i}\right|\right)$, whereas correspondingly the weight of $g_{\boldsymbol{m}}^{X}(\varphi)$ equals $k n+|L|$ (here $|L|=\sum_{i, j} L_{i j}$ ).

Write $M$ for the index of $\varphi_{n}^{\Phi}$ and $Q=Q_{M}$ for the corresponding quadratic form. Recall

$$
\Gamma_{X}=\left\{\gamma \in \mathrm{SL}_{2}(\mathbb{Z}) \mid \gamma X-X \in M_{2, n}(\mathbb{Z}), \rho(X-\gamma X)=\zeta_{X, \gamma X-X}\right\} .
$$

Writing $\gamma=\left(\begin{array}{ll}a & b \\ c & d\end{array}\right)$, we have

$\gamma X-X=(b A,(d-1) A)^{t}, \quad \rho(\gamma X-X)=\boldsymbol{e}\left(\left(b^{2}-b(d-1)+(d-1)^{2}\right) Q(\boldsymbol{a})\right)$

and

$$
\zeta_{X, \gamma X-X}=\boldsymbol{e}(Q(\mathbf{0},(d-1) \boldsymbol{a})-Q(b \boldsymbol{a}, \boldsymbol{a}))=\boldsymbol{e}(-b Q(\boldsymbol{a})) .
$$

\footnotetext{
${ }^{1}$ In fact, for $X=(0, A)$ the double slash operator $\| X$ coincides with the slash operator $\mid X$, so that the "Taylor coefficients" do not involve this family.
} 
Observe that $2 N^{2} Q(\boldsymbol{a})$ is integral. Hence, if $\gamma \in \mathrm{SL}_{2}(\mathbb{Z})$ satisfies

$$
b \equiv 0 \bmod N, \quad d \equiv 1 \bmod N \quad \text { and } \quad b^{2}-b(d-1)+(d-1)^{2} \equiv b \bmod 2 N^{2},
$$

then $\gamma \in \Gamma_{X}$.

Let $N^{\prime} \in \mathbb{Z}_{>0}$. Then,

$$
w_{N^{\prime}}^{-1} \Gamma\left(N^{\prime}\right) w_{N^{\prime}}=\left\{\left(\begin{array}{ll}
a & b \\
c & d
\end{array}\right) \in \mathrm{SL}_{2}(\mathbb{Z}) \mid b \equiv 0\left(N^{\prime 2}\right), a \equiv d \equiv 1\left(N^{\prime}\right)\right\} .
$$

In case $2 \nmid N$, the conditions (6.4) are satisfied for all $\gamma \in w_{N^{\prime}}^{-1} \Gamma\left(N^{\prime}\right) w_{N^{\prime}}$ when $N^{\prime}=N$, in case $2 \mid N$ for $N^{\prime}=2 N$. Therefore, indeed, $w_{M}^{-1} \Gamma(M) w_{M} \leq \Gamma_{X}$.

For a monomial element $f_{L}(A)$ as in (6.3) with $L \in M_{n, r}(\mathbb{Z})$ and $A \in M_{n, r}(\mathbb{Q})$, and $\gamma \in \Gamma_{1}(N)$ one has that

$$
\left\langle f_{L}(A)\right\rangle_{q} \mid \gamma=\boldsymbol{e}\left(\left(b^{2}-b d+(d-1)^{2}\right) Q(\boldsymbol{a})\right)\left\langle f_{L}(A)\right\rangle_{q}
$$

where $Q$ is again the (quadratic form corresponding to the) index of $\varphi_{n}^{\Phi}$. Hence, restricting to $A \in M_{n, r}(\mathbb{Q}) \simeq \mathbb{Q}^{n r}$ for which $Q(A) \in \frac{1}{2} \mathbb{Z}$ we find the following result, from which we are able to derive Theorem 6.1.3 using some additional properties of the Bloch-Okounkov $n$-point functions.

Proposition 6.2.5. Given $N \geq 1$, for all $L \in M_{n, r}(\mathbb{Z})$ and $A \in \frac{1}{N} M_{n, r}(\mathbb{Z})$ satisfying $Q(A) \in \frac{1}{2} \mathbb{Z}+\frac{1}{N} \mathbb{Z}$, one has that $\left\langle f_{L}(A)\right\rangle_{q}$ is a quasimodular form for $\Gamma_{1}(N)$.

Proof. This follows from the observation (6.5) using the following two observations. First of all, $b^{2}-b d+(d-1)^{2} \equiv 0 \bmod N$ when $b \equiv 0, d \equiv 1 \bmod N$. Secondly, for integers $b, d$ the integer $b^{2}-b d+(d-1)^{2}$ is always even whenever not both $b$ and $d$ are even.

The functions $Q_{k}^{(p)}$ in [GJT16] are given by

$$
Q_{k}^{(p)}(\lambda)=\beta_{k}^{(p)}+\sum_{\operatorname{gcd}\left(2 \lambda_{i}-2 i+1, p\right)=1}\left(\left(\lambda_{i}-i+\frac{1}{2}\right)^{k-1}-\left(-i+\frac{1}{2}\right)^{k-1}\right),
$$

where $\beta_{k}^{(p)}=\beta_{k}(0)\left(1-\frac{1}{p}\right)$. Observe that

$$
Q_{k}^{(p)}(\lambda)=Q_{k}(\lambda)-\frac{1}{p} \sum_{a=0}^{p-1} e(a / p) Q_{k}(2 a / p)(\lambda)
$$

Outside of the context of $p$-adic modular forms, there is no need to restrict $p$ to be a prime, so (6.6) serves as the definition of $Q_{k}^{(m)}$ for an integer $m$. Similarly, we define functions $f_{\ell}^{d}$ in terms of the Taylor coefficients $f_{\ell}(\boldsymbol{a})$, as follows. 
Definition 6.2.6. Let $\Phi$ be as above. Given $\boldsymbol{d} \in \mathbb{Z}_{>0}^{r}$, we let

$$
U(\boldsymbol{d})=\left\{0, \frac{1}{d_{1}}, \ldots, \frac{d_{1}-1}{d_{1}}\right\} \times \cdots \times\left\{0, \frac{1}{d_{r}}, \ldots, \frac{d_{r}-1}{d_{r}}\right\}
$$

and for $\ell \in \mathbb{Z}^{r}$ define

$$
f_{\ell}^{\boldsymbol{d}, \Phi}(\lambda):=f_{\ell}^{\boldsymbol{d}}(\lambda):=\sum_{\boldsymbol{a} \in U(\boldsymbol{d})} f_{\ell}^{\Phi}(\lambda, 2 \boldsymbol{a}) \quad(\lambda \in \mathscr{P}) .
$$

Define the graded algebra $\mathcal{F}^{(N), \Phi}$ as the $\mathbb{Q}$-algebra generated by the functions $f_{\ell}^{d, \Phi}$ for all $\ell \in \mathbb{Z}^{r}$ and $\boldsymbol{d} \in \mathbb{Z}_{>0}^{r}$ for which $d_{i} \mid N$ for all $i$.

Then, Theorem 6.1 .5 follows directly from the following result.

Theorem 6.2.7. Let $\Phi$ be as above. Given $N \geq 1$, the algebra $\mathcal{F}^{(N), \Phi}$ is a quasimodular algebra for the congruence subgroup $\Gamma_{0}\left(N^{2}\right)$.

Proof. Consider the monomial elements $f_{L}^{D}:=f_{L_{1}}^{D_{1}} \cdots f_{L_{n}}^{D_{n}}$ in $\mathcal{F}^{(N)}$, where $L, D \in$ $M_{n r}(\mathbb{Z})$. Everywhere in this proof we identify $M_{n, r}(\mathbb{Z})$ with $\mathbb{Z}^{n r}$. Then,

$$
f_{L}^{D}=\sum_{A \in U(D)} f_{L}(\cdot, 2 A)
$$

Now, by part (ii)' in Theorem 5.9.10, for all $\gamma=\left(\begin{array}{ll}a & b \\ c & d\end{array}\right) \in \mathrm{SL}_{2}(\mathbb{Z})$ one has that

$$
\left\langle f_{L}(\cdot, 2 A)\right\rangle_{q} \mid \gamma=\sum_{r} h_{r}(2 b A, 2 d A)\left(\frac{c}{c \tau+d}\right)^{r},
$$

where $h_{r}(\boldsymbol{\lambda}, \boldsymbol{\mu})=\sum_{s} \frac{1}{(r-s) !} \mathcal{Q}^{r-s} g_{\ell, s}^{X}\left(\varphi_{n}\right)$ and $X=\left(\begin{array}{c}\boldsymbol{\lambda} \\ \boldsymbol{\mu}\end{array}\right)$. If $\gamma \in \Gamma_{0}\left(N^{2}\right)$, then $2 b A \in 2 N M_{n, r}(\mathbb{Z})$. Hence, for $X=(0,2 d A)^{t}$ and $X^{\prime}=(2 b A, 0)^{t}$ one has $\rho\left(X^{\prime}\right) \zeta_{X, X^{\prime}}=1$. Therefore,

$$
h_{r}(2 b A, 2 d A)=h_{r}(\mathbf{0}, 2 d A) \quad \text { and } \quad h_{r}(\mathbf{0}, 2 d A+B)=h_{r}(\mathbf{0}, 2 d A)
$$

for all $B \in 2 M_{n, r}(\mathbb{Z})$. As $2 d A$ ranges over the same values modulo 2 as $2 A$ does for $A \in U(D)$, one finds

$$
\left\langle f_{L}^{D}\right\rangle_{q} \mid \gamma=\sum_{r} \sum_{A \in U(D)} h_{r}(\mathbf{0}, 2 A)\left(\frac{c}{c \tau+d}\right)^{r}
$$

for all $\gamma \in \Gamma_{0}\left(N^{2}\right)$. Hence, indeed, $\left\langle f_{L}^{D}\right\rangle_{q}$ is a quasimodular form for $\Gamma_{0}\left(N^{2}\right)$.

The rest of this chapter is devoted to providing examples of quasimodular algebras of higher level, using Theorem 6.2.7. 


\subsection{First application: the Bloch-Okounkov theorem of higher level}

The construction and results on the Bloch-Okounkov algebra, as stated in the introduction, are proven in this section. In fact, this proof is an almost immediate consequence of the results in the previous section using the properties of the BlochOkounkov $n$-point functions.

Recall the Bloch-Okounkov $n$-point functions $F_{n}$ defined in Definition 1.4.2 as follows. For all $n \geq 0$, let $\mathfrak{S}_{n}$ be the symmetric group on $n$ letters and let

$$
F_{n}\left(\tau, z_{1}, \ldots, z_{n}\right)=\sum_{\sigma \in \mathfrak{S}_{n}} V_{n}\left(\tau, z_{\sigma(1)}, \ldots, z_{\sigma(n)}\right),
$$

where the functions $V_{n}$ are defined recursively by $V_{0}(\tau)=1$ and

$$
\sum_{m=0}^{n} \frac{(-1)^{n-m}}{(n-m) !} \theta^{(n-m)}\left(\tau, z_{1}+\ldots+z_{m}\right) V_{m}\left(\tau, z_{1}, \ldots, z_{m}\right)=0 .
$$

These $n$-point functions are quasi-Jacobi forms of which we determine the weight and index (or rather the quadratic form uniquely determining a symmetric matrix $M \in M_{n}(\mathbb{Q})$ which is the index).

Lemma 6.3.1. The n-point functions $F_{n}$ are meromorphic quasi-Jacobi forms of weight $n$ and index $Q(\boldsymbol{z})=-\frac{1}{2}\left(z_{1}+\ldots+z_{n}\right)^{2}$.

Proof. We start with the observation that for all $n \geq 0$ the function $\frac{\Theta^{(n)}(z)}{\Theta(z)}$ is a true meromorphic Jacobi form (of weight $n$ and index $Q(z)=0$ ), in contrast to $\Theta(z)$ itself which is weakly holomorphic. Namely, all poles are given by $z \in \mathbb{Z} \tau+\mathbb{Z}$ and for $z=a+b \tau$ with $a, b \in \mathbb{Q}$, one has that

$$
\frac{\Theta^{(n)}(a \tau+b)}{\Theta(a \tau+b)}=\frac{\sum_{\nu \in \mathbb{F}} \nu^{n} \boldsymbol{e}(\nu b) q^{\nu^{2} / 2+a \nu}}{\sum_{\nu \in \mathbb{F}} \boldsymbol{e}(\nu b) q^{\nu^{2} / 2+a \nu}} \rightarrow-a,
$$

whenever $\tau_{2} \rightarrow \infty$, or equivalently $q \rightarrow 0$.

Next, observe that the product $\Theta\left(z_{1}+\ldots+z_{n}\right) V_{n}\left(z_{1}, \ldots, z_{n}\right)$ can be written as a polynomial of weight $n-1$ in $\frac{\Theta^{(i)}\left(z_{1}+\ldots+z_{j}\right)}{\Theta\left(z_{1}+\ldots+z_{j}\right)}$ for $i, j=1, \ldots, n$; a fact which can be proven inductively by its recursion (1.15). Hence, $\Theta\left(z_{1}+\ldots+z_{n}\right) V_{n}\left(z_{1}, \ldots, z_{n}\right)$ is a meromorphic Jacobi form. As $\Theta(z)^{-1}$ is a meromorphic Jacobi form of weight 1 and index $Q(z, z)=-\frac{1}{2} z^{2}$, we conclude that $F_{n}$ is a meromorphic quasi-Jacobi form of weight $n$ and index $Q(\boldsymbol{z})=-\frac{1}{2}|\boldsymbol{z}|^{2}$.

Observe that Theorem 6.1.1 is a direct corollary of the previous lemma and Theorem 6.2.4. Also, Theorem 6.1.5 follows directly from the previous lemma and Theorem 6.2.7. So, we are only left with the following proof. 
Proof of Theorem 6.1.3. First of all, in case $a \in \mathbb{Z}$, one has $Q(\boldsymbol{a})=-\frac{1}{2}|\boldsymbol{a}|^{2} \in \frac{1}{2} \mathbb{Z}$. Hence, the result follows directly from Proposition 6.2.5.

In the second case, for both $\left\langle Q_{\boldsymbol{k}}(\boldsymbol{a})\right\rangle_{q}$ and $\left\langle Q_{1}(a)\right\rangle_{q}$ the root of unity in (6.5) is $\boldsymbol{e}(Q(\boldsymbol{a}))=\boldsymbol{e}(Q(a))$, so that again the subgroup of quasimodularity is $\Gamma_{1}(N)$.

For the holomorphicity in the second case, observe that $\left\langle Q_{1}(a)\right\rangle_{q}$ equals $\Theta(a)^{-1}$ up to a constant. Also, $\left\langle Q_{\boldsymbol{k}}(\boldsymbol{a})\right\rangle_{q}$ can be written as a product of Taylor coefficients of $\Theta\left(z_{1}+\ldots+z_{n}+a\right)^{-1}$ and of $\Theta\left(z_{1}+\ldots+z_{n}+a\right) F_{n}\left(z_{1}+a_{1}, \ldots, z_{n}+a_{n}\right)$, the latter Taylor coefficients being holomorphic quasimodular forms. Observe that the Taylor coefficients of

$$
\frac{\Theta(a)}{\Theta\left(z_{1}+\ldots+z_{n}+a\right)}
$$

around $z_{1}=\ldots=z_{n}=0$ are all polynomials in the holomorphic quasimodular forms $\frac{\Theta^{(i)}(a)}{\Theta(a)}$. Therefore, $\frac{\left\langle Q_{k}(a)\right\rangle_{q}}{\left\langle Q_{1}(a)\right\rangle_{q}}$ is a holomorphic quasimodular form.

\subsection{Second application: hook-length moments of higher level}

As a second example, recall the hook-length moments, defined by (1.12). The results in Section 6.2 specialise to the following statements.

(i). For $a \in \mathbb{Q}$ and $k \in \mathbb{Z}_{\geq 2}$, let

$$
H_{k}(a):=\tilde{\alpha}_{k}(a)+\frac{1}{2} \sum_{\xi \in Y_{\lambda}}\left(\boldsymbol{e}(a h(\xi))+(-1)^{k} \boldsymbol{e}(-a h(\xi))\right) h(\xi)^{k-2},
$$

where $\sum_{k} \tilde{\alpha}_{k}(a) \frac{z^{k-2}}{(k-2) !}=\frac{1}{8} \sinh ((z+2 \pi \mathrm{i} a) / 2)^{-2}$ (here $(-2) !=1$ ). Denote by $\mathcal{H}(N)$ the graded algebra generated by the $H_{k}(a)$ with $k \geq 2$ and $N a \in \mathbb{Z}$. The algebra $\mathcal{H}(N)$ is graded by assigning to $H_{k}(a)$ weight $k$. Let $M=(2, N) N$.

Corollary 6.4.1. The algebra $\mathcal{H}(N)$ is quasimodular of level $M$, after scaling $\tau$ by $M$.

More concretely, for all homogeneous $f \in \mathcal{H}(N)$ of weight $k$, the rescaled $q$ bracket $\langle f\rangle_{q_{M}}$, where $q_{M}=q^{1 / M}$, is a quasimodular form of weight $k$ for $\Gamma(M)$. 
(ii). Given $N \geq 1, \boldsymbol{k} \in \mathbb{Z}^{n}$ and $\boldsymbol{a} \in \frac{1}{N} \mathbb{Z}^{n}$, write $H_{\boldsymbol{k}}(\boldsymbol{a})=H_{k_{1}}\left(a_{1}\right) \cdots H_{k_{n}}\left(a_{n}\right)$.

Corollary 6.4.2. For $\boldsymbol{a} \in \frac{1}{N} \mathbb{Z}^{n}$ with $|\boldsymbol{a}| \in \mathbb{Z}$ and $\boldsymbol{k} \in \mathbb{Z}_{\geq 2}^{n}$ the q-bracket $\left\langle H_{\boldsymbol{k}}(\boldsymbol{a})\right\rangle_{q}$ is a quasimodular form of weight $|\boldsymbol{k}|$ for $\Gamma_{1}(N)$.

(iii). For $k, t \in \mathbb{Z}_{\neq 0}$, let

$$
H_{k}^{t}:=-\frac{B_{k}}{2 k} t^{k}+\sum_{\substack{\xi \in Y_{\lambda} \\ h(\xi) \equiv 0 \bmod t}} h(\xi)^{k-2},
$$

which (up to a constant) also occurs in [BOW20]. Denote by $\mathcal{H}^{(N)}$ the algebra generated by the $H_{k}^{t}$ for which $k$ is even and $t \mid N$. This algebra is graded by assigning weight $k$ to $H_{k}^{t}$.

Corollary 6.4.3. The algebra $\mathcal{H}^{(N)}$ is quasimodular for $\Gamma_{0}\left(N^{2}\right)$.

More concretely, for all homogeneous $f \in \mathcal{H}^{(N)}$ of weight $k$, the $q$-bracket $\langle f\rangle_{q}$ is a quasimodular form of weight $k$ for $\Gamma_{0}\left(N^{2}\right)$.

\subsection{Third application: moment functions of higher level}

Next, we consider the moment functions $S_{k}$ of Chapter 4. By Corollary 4.5.2, the generating series $\mathscr{S}(z)=\frac{1}{2 z^{2}}+\sum_{k \geq 2} S_{k} \frac{z^{k-2}}{(k-2) !}$ satisfies

$$
\left\langle\mathscr{S}\left(z_{1}\right) \cdots \mathscr{S}\left(z_{n}\right)\right\rangle_{q}=\frac{1}{2^{n+1}} \sum_{\alpha \in \Pi(n)} \prod_{A \in \alpha} \sum_{\boldsymbol{s} \in\{-1,1\}|A|} D_{\tau}^{|A|-1} E_{2}\left(\boldsymbol{s} \cdot \boldsymbol{z}_{A}\right),
$$

where $\Pi(n)$ denotes the set of all set partitions of the set $\{1, \ldots, n\},|A|$ the cardinality of the set $A$, and $z_{A}=\left(z_{a_{1}}, \ldots, z_{a_{r}}\right)$ if $A=\left\{a_{1}, \ldots, a_{r}\right\}$. Hence, the $n$-point functions (6.7) are quasi-Jacobi forms of weight $2 n$ and index zero. Because the index of the quasi-Jacobi forms (6.7) is zero, the root of unity in (6.5) vanishes for all $\gamma \in \mathrm{SL}_{2}(\mathbb{Z})$. Therefore, the results of Section 6.2 specialise to the following results for the groups $\Gamma_{1}(N)$ and $\Gamma_{0}(N)$ (rather than $\Gamma(N)$ and $\Gamma_{0}\left(N^{2}\right)$, respectively). 
(i). For $a \in \mathbb{Q}$ and $k \geq 1$, let

$$
S_{k}(a):=\alpha_{k}(a)+\frac{1}{2} \sum_{i=1}^{\infty}\left(\boldsymbol{e}\left(a \lambda_{i}\right)+(-1)^{k} \boldsymbol{e}\left(-a \lambda_{i}\right)\right) \lambda_{i}^{k-1},
$$

where $\sum_{k} \alpha_{k}(a) \frac{(2 \pi \mathrm{i} z)^{k-1}}{(k-1) !}=\frac{1}{2}(\boldsymbol{e}(z+a)-1)^{-1}$ (here $(-1) !=1$; for $k \geq 2$, these values agree with the constants $\tilde{\alpha}_{k}$ in the previous example). Denote by $\mathcal{S}(N)$ the algebra generated by the $S_{k}(a)$ with $N a \in \mathbb{Z}$. Assign to $S_{k}(a)$ weight $k$.

Corollary 6.5.1. The algebra $\mathcal{S}(N)$ is quasimodular for $\Gamma_{1}(N)$.

More concretely, for all homogeneous $f \in \mathcal{H}(N)$ of weight $k$, the $q$-bracket $\langle f\rangle_{q}$ is a quasimodular form of weight $k$ for $\Gamma_{1}(N)$.

(ii). For $k, t \in \mathbb{Z}_{>0}$, let

$$
S_{k}^{t}:=-\frac{B_{k}}{2 k} t^{k}+\sum_{\substack{i \geq 0 \\ \lambda_{i} \equiv 0 \bmod t}} \lambda_{i}^{k-1} .
$$

Denote by $\mathcal{S}^{(N)}$ the algebra generated by the $S_{k}^{t}$ for which $k$ is even and $t \mid N$. Assign to $S_{k}^{t}$ weight $k$.

Corollary 6.5.2. The algebra $\mathcal{S}^{(N)}$ is quasimodular for $\Gamma_{0}(N)$.

More concretely, for all homogeneous $f \in \mathcal{S}^{(N)}$ of weight $k$, the $q$-bracket $\langle f\rangle_{q}$ is a quasimodular form of weight $k$ for $\Gamma_{0}(N)$.

\subsection{Fourth application: double moment functions of higher level}

As a final example, consider the double moment functions $T_{k, l}$, defined by (4.2). By Theorem 4.8.11, the generating series $\mathscr{T}(z)=-\frac{1}{2 u}-\frac{1}{2 v}+\sum_{k, \ell} T_{k, l} \frac{u^{k} v^{\ell-1}}{(k) !(\ell-1) !}$ satisfies

$$
G_{1}(u, v):=\langle\mathscr{T}(u, v)\rangle_{q}=-\frac{1}{2} \frac{\Theta(u+v)}{\Theta(u) \Theta(v)} .
$$

Hence, the 1-point function $G_{1}$ is a Jacobi form of weight 1 and index $u v$. This example now deviates from the previous ones because $\left\langle\mathscr{T}\left(u_{1}, v_{1}\right) \cdots \mathscr{T}\left(u_{n}, v_{n}\right)\right\rangle_{q}$ is not a Jacobi form of some fixed weight (but of mixed weight). With respect to the induced product $\odot$, defined by Definition 4.4.1, one finds

$$
G_{n}(\boldsymbol{u}, \boldsymbol{v}):=\left\langle\mathscr{T}\left(u_{1}, v_{1}\right) \odot \cdots \odot \mathscr{T}\left(u_{n}, v_{n}\right)\right\rangle_{q}=\prod_{i=1}^{n}\left(-\frac{1}{2} \frac{\Theta\left(u_{i}+v_{i}\right)}{\Theta\left(u_{i}\right) \Theta\left(v_{i}\right)}\right) .
$$


Observing that in Section 6.2 one can replace the pointwise product on functions of partitions by the induced product, we have the following generalisations of the algebra $\mathcal{T}$.

(i). For $a, b \in \mathbb{Q}$, and $k \geq 0, \ell \geq 1$, let $T_{k, \ell}(a, b)$ be given by

$$
C_{k, \ell}(a, b)+\sum_{m=1}^{\infty} m^{k}\left(\boldsymbol{e}(a m) \mathcal{F}_{\ell}^{b}\left(r_{m}(\lambda)\right)+(-1)^{k+l} \boldsymbol{e}(-a m) \mathcal{F}_{\ell}^{-b}\left(r_{m}(\lambda)\right)\right),
$$

where

$$
C_{k, \ell}(a, b)= \begin{cases}\alpha_{k}(a) & \ell=1 \\ \alpha_{\ell-1}(b) & k=0 \\ 0 & \text { else }\end{cases}
$$

with the constants $\alpha_{k}$ are defined in the previous section. Also, $\mathcal{F}_{\ell}^{b}$ is the polynomial of degree $\ell-1$ (or of degree $\ell$ if $b \in \mathbb{Z}$ ) given by $\mathcal{F}_{\ell}^{b}(n)=\sum_{i=1}^{n} \boldsymbol{e}(b i) i^{l-1}$ for all $n \in \mathbb{Z}_{>0}$. Let the $\mathbb{Q}$-algebra $\mathcal{T}(N)$ be generated by the functions $T_{k, \ell}(a, b)$, where $a, b \in \frac{1}{N} \mathbb{Z}$, under the induced product. Assign to $T_{k, \ell}(a, b)$ weight $k+\ell$ and extend to a weight grading under the induced product. Let $M=(2, N) N$.

Corollary 6.6.1. The algebra $\mathcal{T}(N)$ is quasimodular of level $M$, after scaling $\tau$ by $M$.

More concretely, for all homogeneous $f \in \mathcal{T}(N)$ of weight $k$, the rescaled $q$ bracket $\langle f\rangle_{q_{M}}$, where $q_{M}=q^{1 / M}$, is a quasimodular form of weight $k$ for $\Gamma(M)$.

(ii). Let $\boldsymbol{k}, \boldsymbol{\ell} \in \mathbb{Z}^{n}$ and $\boldsymbol{a}, \boldsymbol{b} \in \frac{1}{N} \mathbb{Z}^{n}$.

Corollary 6.6.2. Whenever $\boldsymbol{a} \cdot \boldsymbol{b} \in \frac{1}{2} \mathbb{Z}+\frac{1}{N} \mathbb{Z}$, one has that

$$
\left\langle T_{k_{1}, \ell_{1}}\left(a_{1}, b_{1}\right) \odot \cdots \odot T_{k_{n}, \ell_{n}}\left(a_{n}, b_{n}\right)\right\rangle_{q}
$$

is a quasimodular form of weight $|\boldsymbol{k}|+|\ell|$ for $\Gamma_{1}(N)$.

(iii). For $k, \ell, s, t \in \mathbb{Z}_{>0}$ let

$$
T_{k, \ell}^{s, t}(\lambda):=C_{k, \ell}+\sum_{m} m^{k} \mathcal{F}_{\ell}\left(\left\lfloor\frac{r_{m s}(\lambda)}{t}\right\rfloor\right) .
$$

Denote by $\mathcal{T}^{(N)}$ the algebra generated by the $T_{k, \ell}^{s, t}$, where $k, \ell$ are even and $s, t \mid N$, under the induced product. Assign to $T_{k, \ell}^{s, t}$ weight $k+\ell$ and extend to a weight grading under the induced product. 
Corollary 6.6.3. The algebra $\mathcal{T}^{(N)}$ is quasimodular for $\Gamma_{0}\left(N^{2}\right)$.

More concretely, for all homogeneous $f \in \mathcal{H}^{(N)}$ of weight $k$, the $q$-bracket $\langle f\rangle_{q}$ is a quasimodular form of weight $k$ for $\Gamma_{0}\left(N^{2}\right)$.

Remark 6.6.4. Combining this result with Corollary 6.6.2, by restricting to $t=1$, we find that any polynomial in $T_{k, \ell}^{s, 1}$ with respect to the induced product is quasimodular for $\Gamma_{0}(N)$. A similar statement holds after restricting to $s=1$. 


\section{When is the q-bracket modular?}

\subsection{Introduction}

For all $k \geq 0$, let $h_{k} \in \Lambda^{*}$ be given by

$$
h_{k}(\lambda)=\sum_{r=0}^{\left\lfloor\frac{k}{2}\right\rfloor} \frac{Q_{2}(\lambda)^{r} Q_{k-2 r}(\lambda)}{2^{r}\left(k-r-\frac{3}{2}\right)_{r} r !} \quad\left((x)_{n}=x(x+1) \cdots(x+n-1)\right) .
$$

We show that the function $h_{k}$ satisfies the following three properties:

(i) The $q$-bracket $\left\langle h_{k}\right\rangle_{q}$ is a modular form (and not just a quasimodular form);

(ii) The difference $h_{k}-Q_{k}$ is divisible by $Q_{2}$;

(iii) For $f \in \Lambda^{*}$ with $\langle f\rangle_{q}$ modular and $f-Q_{k}$ divisible by $Q_{2}$, we have

$$
\langle f\rangle_{q}=\left\langle h_{k}\right\rangle_{q}
$$

Hence, one can think of the difference $h_{k}-Q_{k}$ as a correction term for $Q_{k}$ with respect to the property of being a modular form under the $q$-bracket. By the third property this correction term is unique up to elements in the kernel of the $q$-bracket. More generally, the question "when is the $q$-bracket of $f \in \Lambda^{*}$ modular?" is answered by Theorem A. The functions $h_{k}$ above provide part of the data of the computable projection $\pi$ in this result. By exploring the definition of certain modular forms associated to the quasi-Jacobi forms $F_{n}$, we provide a different method to construct the harmonic shifted symmetric functions $h_{\lambda}$ (of which $h_{k}$ is a special case), already given in in Chapter 2, where $\lambda$ is a partition. The method of proof in this chapter (i.e., using the quasi-Jacobi forms $F_{n}$ ) now allows us to generalise the above result to different settings.

This chapter, as well the previous two chapters, are based on [I21b]. 
We first state and prove our answer to the question in the title of this chapter in full generality, using the main result on the Taylor coefficients of quasi-Jacobi forms (Theorem 5.9.10). Afterwards we provide many examples. In particular, we prove the results on the 'modular subspace' of the Bloch-Okounkov algebra as stated in the introduction, and state similar results for the Bloch-Okounkov algebra for congruence subgroups as well as the algebra of double moment functions.

\subsection{Construction of functions with modular $q$-bracket}

Given a quasi-Jacobi form $\varphi$ of weight $k$ satisfying the conditions of Theorem 5.9.10, the functions

$$
\xi_{\ell}(\varphi)=\sum_{r}(-1)^{r} \sum_{s \leq r} \frac{\left(D_{\tau}+\mathbb{e}_{2}\right)^{r} \mathcal{Q}^{r-s} g_{\ell, s}(\varphi)}{\left(k+|\ell|-r-\frac{3}{2}\right)_{r}(r-s) !},
$$

where $\mathbb{e}_{2}$ is given by Equation 5.12, and $\mathcal{Q}^{r-s} g_{\ell, s}(\varphi)$ in Definition 5.9.7, are modular forms (see also Remark 5.9.8). Therefore, as in the previous section, assume that $\Phi$ : $\mathscr{P} \times \mathbb{C}^{r} \rightarrow \mathbb{C}$ is such that for all $n \geq 1$ there exists a weight $k \in \mathbb{Z}$ such that the function $\varphi_{n}^{\Phi}: \mathfrak{h} \times M_{n, r}(\mathbb{C}) \rightarrow \mathbb{C}$ given by

$$
\varphi_{n}^{\Phi}(\tau, Z):=\left\langle\prod_{i=1}^{n} \Phi\left(\cdot, Z_{i}\right)\right\rangle_{q},
$$

where $Z_{i}$ is the $i$ th row of $Z$, is a meromorphic quasi-Jacobi form of weight $k n$ which admits a Laurent expansion around all $Z \in M_{n, r}(\mathbb{Q})$ (after identifying $M_{n, r}(\mathbb{C}$ ) with $\mathbb{C}^{n r}$ ). Write $\mathcal{F}=\mathcal{F}^{\Phi}(1)$ for the graded algebra of Taylor coefficients of $\Phi$ (see Definition 6.2.2). Given $\ell \in \mathbb{Z}^{n}$, our aim is to find $h_{\ell} \in \mathcal{F}$ such that $\left\langle h_{\ell}\right\rangle_{q}=\xi_{\ell}\left(\varphi_{n}\right)$, i.e., we want to determine a pull back of $\xi_{\ell}\left(\varphi_{n}\right)$ under the $q$-bracket.

In order to do so, assume the algebra $\mathcal{F}$ satisfies the following two properties:

(i) $Q_{2} \in \mathcal{F}$,

(ii) There is a linear operator $\mathscr{D}$ acting on $\mathcal{F}$ such that

$$
\left\langle\mathscr{D} \prod_{i=1}^{n} \Phi\left(\boldsymbol{z}^{i}\right)\right\rangle_{q}=\left(\mathfrak{d}_{\tau}+z_{1} \mathfrak{d}_{z_{1}}+\ldots+z_{n} \mathfrak{d}_{z_{n}}\right) \varphi,
$$

where $\mathscr{D}$ is extended to a linear operator on $\mathcal{F}\left[\left[z_{1}, \ldots, z_{n}\right]\right]$ by $\mathscr{D}\left(f \boldsymbol{z}^{\ell}\right)=$ $\mathscr{D}(f) \boldsymbol{z}^{\ell}$ for all $f \in \mathcal{F}$ and $\ell \in \mathbb{Z}^{n}$. 
Remark 7.2.1. The function $Q_{2}$ is known to make the $q$-bracket equivariant with respect to the operator $D_{\tau}+\mathbb{e}_{2}$, i.e.,

$$
\left\langle Q_{2} f\right\rangle_{q}=\left(D+\mathbb{e}_{2}\right)\langle f\rangle_{q}
$$

for all $f: \mathscr{P} \rightarrow \mathbb{C}$, explaining the first condition. The second condition is explained by noting that

$$
g_{\ell, s}(\varphi)=g_{\ell}\left(\sum_{i+|\boldsymbol{j}|=s} \varphi_{i, \boldsymbol{j}} z^{j}\right)=\frac{1}{s !} g_{\ell}\left(\left(\mathfrak{d}_{\tau}+z_{1} \mathfrak{d}_{z_{1}}+\ldots+z_{n} \mathfrak{d}_{z_{n}}\right)^{s} \varphi\right) .
$$

Recall that the algebra $\mathcal{F}=\mathcal{F}^{\Phi}(1)$ is generated by the Taylor coefficients $f_{\ell}=$ $f_{\ell}(\mathbf{0})$ of $\Phi$ for $\ell \in \mathbb{Z}^{r}$. An arbitrary monomial $f_{L}$ in $\mathcal{F}$ is given by $f_{L_{1}} \cdots f_{L_{n}}$ with $L \in M_{n, r}(\mathbb{Z})$. We define an operator on $\mathcal{F}$ corresponding to the index of $\varphi$.

Definition 7.2.2. Let $M=\left(m_{i, j}\right) \in M_{n}(\mathbb{Q})$ be the index of $\varphi_{n}^{\Phi}$. Let $\mathscr{Q}$ be the linear operator on $\mathcal{F}$ which is given on monomials by

$$
\mathscr{Q} f_{L}=\sum_{i, j} m_{i, j} f_{L-e_{i}-e_{j}} \quad\left(L \in M_{n, r}(\mathbb{Z}) \simeq \mathbb{Z}^{n r}\right),
$$

where, on the right hand side, $e_{i}$ is a unit vector in $\mathbb{Z}^{n r}$.

Now, Theorem G can more explicitly be stated as follows, where the three properties below should be compared with the three properties satisfied by the functions $h_{k}$ in the introduction (Section 7.1).

Theorem 7.2.3 (= Theorem $\mathrm{G}$ ). Let $\mathcal{F}$ be a quasimodular algebra satisfying the above conditions, and $\mathscr{Q}, \mathscr{D}: \mathcal{F} \rightarrow \mathcal{F}$ the operators defined by Definition 7.2.2 and Equation 7.1, respectively. Then, the linear mapping $\pi: \mathcal{F} \rightarrow \mathcal{F}$ given by

$$
\pi(f)=\sum_{r \geq 0} \sum_{s=0}^{r}(-1)^{r} \frac{Q_{2}^{r} \mathscr{Q}^{r-s} \mathscr{D}^{s} f}{\left(m-r-\frac{3}{2}\right)_{r}(r-s) ! s !}
$$

whenever $f \in \mathcal{F}$ is homogeneous of weight $m$ satisfies

$$
\langle\pi(\mathcal{F})\rangle_{q} \subseteq M .
$$

Furthermore, one can choose a vector subspace $\mathcal{M} \subseteq \pi \mathcal{F}$ such that

(i) $\mathcal{F}=\mathcal{M} \oplus Q_{2} \mathcal{F}$;

(ii) $\langle\mathcal{M}\rangle_{q} \subseteq M$;

(iii) $\left\langle Q_{2} \mathcal{F}\right\rangle_{q} \cap M=\{0\}$. 
Remark 7.2.4. For the Bloch-Okounkov algebra $\Lambda^{*}$ the mapping $\pi$ turns out be the projection of Theorem A and $\mathcal{M}=\pi\left(\Lambda^{*}\right)=\mathcal{H}$. We do not expect that the conditions in this section ensure that $\pi$ is a projection in general, nor that $\pi\left(Q_{2} \mathcal{F}\right)=\{0\}$ (in which case one could choose $\mathcal{M}=\pi(\mathcal{F})$ ), nor that the splitting in (i) is canonical. However, just as with Theorem $\mathrm{A}$, once one has chosen $\mathcal{M}$ it follows immediately from (i) that every element of $\mathcal{F}$ has a canonical expansion

$$
f=\sum_{i \geq 0} f_{i} Q_{2}^{i}
$$

with $f_{i} \in \mathcal{M}$, and that $\langle f\rangle_{q} \in M$ precisely if $\left\langle f_{i}\right\rangle_{q}=0$ for all $i>0$.

Proof. By (7.2) and by construction of $\mathscr{Q}$ one has that

$$
\frac{1}{s !}\left\langle\mathscr{Q}^{r-s} \mathscr{D}^{s} f_{\boldsymbol{m}}\right\rangle_{q}=\left(\mathcal{Q}^{r-s} g_{\boldsymbol{m}}\right)(\varphi)_{s},
$$

from which it follows that

$$
\left\langle h\left(f_{m}\right)\right\rangle_{q}=\xi_{m}(\varphi),
$$

where $\varphi$ is the meromorphic Jacobi form $\varphi(\boldsymbol{z})=\left\langle\prod_{i=1}^{n} \Phi\left(\boldsymbol{z}^{i}\right)\right\rangle_{q}$. Hence, $\pi(f)$ is modular under the $q$-bracket for all $f \in \mathcal{F}$.

Choose $\mathcal{M}^{0} \subseteq \mathcal{F}$ such that $\mathcal{F}=\mathcal{M}^{0} \oplus Q_{2} \mathcal{F}$. Then, we take $\mathcal{M}=\pi \mathcal{M}^{0}$. As, by definition, $\pi(f)-f$ is a multiple of $Q_{2}$, the first property follows. The second property is immediate as $\mathcal{M} \subseteq \pi(\mathcal{F})$. For the last property, let $f \in Q_{2} \mathcal{F}$ with $\langle f\rangle_{q} \in M$ be given. As $f$ is divisible by $Q_{2}$, the $q$-bracket $\langle f\rangle_{q}$ is in the image of $D+\mathbb{e}_{2}$ acting on quasimodular forms. Now, the zero function is the only function in the image of $D+\mathbb{E}_{2}$ which is modular, so that the last property follows.

The rest of this chapter is devoted to examples of quasimodular algebras to which Theorem 7.2.3 applies.

\subsection{First example: The Bloch-Okounkov algebra}

In order to apply the results of the previous section to the Bloch-Okounkov algebra $\Lambda^{*}$, we have to understand how the operators $\mathfrak{d}_{\tau}$ and $\mathfrak{d}_{z_{i}}$ act on the $n$-points functions $F_{n}$ (defined by Definition 1.4.2), or equivalently, we have to understand the transformation behaviour of $F_{n}$. This behaviour is uniquely determined by the following two properties. 
Proposition 7.3.1. For all $n \geq 1$ one has

$$
\begin{aligned}
\mathfrak{d}_{\tau} F_{n}\left(z_{1}, \ldots, z_{n}\right) & =0 \\
\mathfrak{d}_{z_{1}} F_{n}\left(z_{1}, \ldots, z_{n}\right) & =\sum_{i=2}^{n} F_{n-1}\left(z_{1}+z_{i}, z_{2}, \ldots, \hat{z}_{i}, \ldots, z_{n}\right) .
\end{aligned}
$$

Proof. The second equality is equivalent to [BO00, Theorem 0.6], whereas the first statement seems not to be in the literature. As both statements follow by more or less the same argument, we give both proofs. That is, we prove

$$
\begin{aligned}
\mathfrak{d}_{\tau} V_{n}\left(z_{1}, \ldots, z_{n}\right) & =0, \\
\mathfrak{d}_{z_{i}} V_{n}\left(z_{1}, \ldots, z_{n}\right) & = \begin{cases}V_{n-1}\left(z_{1}, \ldots, z_{i}+z_{i+1}, \ldots, z_{n}\right) & i<n \\
0 & i=n\end{cases}
\end{aligned}
$$

inductively using the recursion (1.15), from which the proposition follows directly.

For $n=1$ both statements are clearly true. Hence, by the identity

$$
\left[\mathfrak{d}_{\tau}, D_{z}^{m}\right]=-2 m D_{z}^{m-1} \mathfrak{d}_{z}+m(m-1) D_{z}^{m-2} .
$$

and after assuming that $\mathfrak{d}_{\tau} V_{n}=0$, we find that $\theta\left(z_{1}+\ldots+z_{n+1}\right) \mathfrak{d}_{\tau} V_{n+1}(\boldsymbol{z})$ equals

$$
-\sum_{m=0}^{n-1} \frac{(-1)^{n-1-m}}{(n-1-m) !} \theta^{(n-1-m)}\left(z_{1}+\ldots+z_{m}\right) V_{m}\left(z_{1}, \ldots, z_{m}\right) .
$$

By the recursion (1.15) one finds that this expression vanishes. Hence, $\mathfrak{d}_{\tau} V_{n+1}=0$ and $\mathfrak{d}_{\tau} F_{n}=0$ as desired.

Denote

$$
V_{m-1}^{i}\left(z_{1}, \ldots, z_{m}\right)=V_{m-1}\left(z_{1}, \ldots, z_{i}+z_{i+1}, \ldots, z_{m}\right) .
$$

By applying $\mathfrak{d}_{z_{i}}$ to the recursion (1.15), using the identity $\left[\mathfrak{d}_{z}, D_{z}^{m}\right]=-2 m D_{z}^{m-1} I$ (with $I$ the index operator, see Remark 5.7.3) and assuming that (7.3) holds, we find

$$
\begin{aligned}
\theta\left(z_{1}+\ldots\right. & \left.+z_{n+1}\right) \mathfrak{d}_{z_{i}} V_{n+1}\left(z_{1}, \ldots, z_{n+1}\right) \\
= & \sum_{m=i}^{n} \frac{(-1)^{n-m}}{(n-m) !} \theta^{(n-m)}\left(z_{1}+\ldots+z_{m}\right) V_{m}\left(z_{1}, \ldots, z_{m}\right)+ \\
& \quad-\sum_{m=i+1}^{n} \frac{(-1)^{n+1-m}}{(n+1-m) !} \theta^{(n+1-m)}\left(z_{1}+\ldots+z_{m}\right) V_{m-1}^{i}\left(z_{1}, \ldots, z_{m}\right) .
\end{aligned}
$$


Again using the recursion (1.15), we conclude that

$$
\begin{aligned}
\theta\left(z_{1}+\ldots\right. & \left.+z_{n+1}\right) \mathfrak{d}_{z_{i}} V_{n+1}\left(z_{1}, \ldots, z_{n+1}\right) \\
= & -\sum_{m=0}^{i-1} \frac{(-1)^{n-m}}{(n-m) !} \theta^{(n-m)}\left(z_{1}+\ldots+z_{m}\right) V_{m}\left(z_{1}, \ldots, z_{m}\right)+ \\
& \quad-\sum_{m=i+1}^{n} \frac{(-1)^{n+1-m}}{(n+1-m) !} \theta^{(n+1-m)}\left(z_{1}+\ldots+z_{m}\right) V_{m-1}^{i}\left(z_{1}, \ldots, z_{m}\right) \\
= & \theta\left(z_{1}+\ldots+z_{n+1}\right) V_{n}\left(z_{1}, \ldots, z_{i}+z_{i+1}, \ldots, z_{n+1}\right) .
\end{aligned}
$$

Remark 7.3.2. By this proposition and Theorem 5.9.10 the transformation of the Taylor coefficients of $F_{n}$ are determined. For its Fourier expansion see [BM15].

Next, recall the $j$ th order differential operators $\mathscr{D}_{j}$, defined in Definition 2.3.8, given by

$$
\mathscr{D}_{j}=\sum_{\boldsymbol{i} \in \mathbb{Z}_{\geq 0}^{j}}\left(\begin{array}{c}
|\boldsymbol{i}| \\
i_{1}, i_{2}, \ldots, i_{j}
\end{array}\right) Q_{|\boldsymbol{i}|} \partial_{\boldsymbol{i}}, \quad \text { with } \quad \partial_{\boldsymbol{i}}=\frac{\partial^{j}}{\partial Q_{i_{1}+1} \cdots \partial Q_{i_{j}+1}},
$$

where the coefficient is a multinomial coefficient (in this section we pretend these operators act on $\Lambda^{*}$, although formally there are only defined on $\mathcal{R}$ by Definition 2.3.8).

These operators turn out to correspond to certain symmetric powers of the derivative operators $\mathfrak{d}_{z_{i}}$. In particular, observe that the coefficient of $\boldsymbol{z}^{\ell}$ in the case $j=1$ below, is given by $g_{\ell}\left(F_{n}\right)_{1}$. Hence, the operator $\mathscr{D}$, requested in the previous section, is given by $\mathscr{D}=\mathscr{D}_{2} / 2$.

Proposition 7.3.3. For all $j \geq 1$ one has

$$
\left\langle\mathscr{D}_{j} W(\boldsymbol{z})\right\rangle_{q}=j !\left(z_{1} \mathfrak{d}_{z_{1}}^{j-1}+\ldots+z_{n} \mathfrak{d}_{z_{n}}^{j-1}\right) F_{n}(\boldsymbol{z}) .
$$

Proof. Observe that

$$
\mathscr{D}_{j} Q_{\ell}=j ! \sum_{i_{1}<\ldots<i_{j}}\left(\begin{array}{c}
\ell_{i_{1}}+\ldots+\ell_{i_{j}}-j \\
\ell_{i_{1}}-1, \ldots, \ell_{i_{j}}-1
\end{array}\right) Q_{\ell_{i_{1}}+\ldots+\ell_{i_{j}}-j} \cdots \widehat{Q}_{l_{i_{1}}} \ldots \widehat{Q}_{l_{i_{j}}} \ldots,
$$

where the binomial coefficient vanishes whenever $l_{i}=0$ for some $i$. Hence,

$$
\mathscr{D}_{j} W_{\lambda}(\boldsymbol{z})=j ! \sum_{i_{1}<\ldots<i_{j}}\left(z_{i_{1}}+\ldots+z_{i_{j}}\right) W_{\lambda}\left(z_{i_{1}}+\ldots+z_{i_{j}}, \ldots, \hat{z}_{i_{1}}, \ldots, \hat{z}_{i_{j}}, \ldots\right) .
$$

By symmetry of $F_{n}$ and Proposition 7.3.1 the statement follows. 
Now, by invoking Theorem 7.2.3, we find the following refinement of Theorem G. In particular, we find a different expression for the projection $\pi$ in Theorem A, and hence also for the basis elements $h_{\lambda}$ in (2.8), which equal $\pi\left(Q_{\lambda_{1}} \ldots Q_{\lambda_{n}}\right)$. Moreover, by construction, we deduce that the $q$-bracket of $h_{\lambda}$ is given by $\xi_{\lambda^{+}}\left(F_{n}\right)$, where $\lambda^{+}=\left(\lambda_{1}+1, \lambda_{2}+1, \ldots\right)$.

Theorem 7.3.4. Let $\partial=\mathscr{D}_{1}$ and $\mathscr{D}_{2}$ be given by Equation 7.4. The linear mapping $\pi: \Lambda^{*} \rightarrow \Lambda^{*}$ given by

$$
\pi(f)=\sum_{r \geq 0} \sum_{s=0}^{r}(-1)^{s} \frac{Q_{2}^{r} \partial^{2 r-2 s} \mathscr{D}_{2}^{s}(f)}{2^{r}\left(\ell-r-\frac{3}{2}\right)_{r}(r-s) ! s !}
$$

whenever $f$ is of weight $\ell$ is a projection satisfying $\pi\left(Q_{2} \Lambda^{*}\right)=0$ and

$$
\pi(f)=\frac{Q_{2}^{-3 / 2+\ell} f^{\vee} Q_{2}^{3 / 2}}{\left(\frac{3}{2}\right)_{\ell}^{-} \ell !},
$$

where the mapping $\vee$ is given on page 34 in terms of the commuting family of operators $\Delta_{\lambda}$ of Definition 2.3.10. Furthermore, $\mathcal{M}:=\pi\left(\Lambda^{*}\right)$ satisfies

(i) $\Lambda^{*}=\mathcal{M} \oplus\left(Q_{2}\right)$, where $\left(Q_{2}\right)=Q_{2} \Lambda^{*}$;

(ii) $\langle\mathcal{M}\rangle_{q} \subseteq M$;

(iii) $\left\langle\left(Q_{2}\right)\right\rangle_{q} \cap M=\{0\}$;

(iv) $\mathcal{M}=\mathcal{H}$, where $\mathcal{H}$ is the harmonic subspace of $\Lambda^{*}$ (see Definition 2.3.1).

Proof. Equation 7.5, as well as the fact that $\pi$ is a projection, follows directly from Corollary 2.3.15. In particular, as $\Delta_{2}\left(Q_{2}^{3 / 2}\right)=0$, it follows that $\pi\left(Q_{2} f\right)=0$.

For the properties of $\mathcal{M}$, observe that $\mathscr{D}$ and $\mathscr{Q}$, defined in the previous section, can also be expressed as $\mathscr{D}_{2} / 2$ and $-\partial^{2} / 2$ respectively. Hence, the first three properties follows from Theorem 7.2.3, and the fourth follows as $\pi$ coincides with the projection map in Theorem A.

Remark 7.3.5. Note that this proof gives a second proof of Theorem A, as for the proof of the first three properties of $\mathcal{M}$ we did not use any of the results of Chapter 2. 


\subsection{Second example: the Bloch-Okounkov algebra of higher level}

In order to extend the result in the previous section to the Bloch-Okounkov algebras $\Lambda^{*}(N)$ of level $N$ (see (6.2) and Section 6.3), we should generalise the operators $\partial$ and $\mathscr{D}_{2}$.

Definition 7.4.1. Let $\widehat{\mathcal{R}}=\mathbb{Q}\left[Q_{k}(a) \mid k \in \mathbb{Z}, a \in \mathbb{Q}\right]$ be the algebra in the formal variables $Q_{k}(a)$ with canonical projection to $\bigcup_{N \in \mathbb{Z}} \Lambda^{*}(N)$. Given $\boldsymbol{a} \in \mathbb{Q}^{j}$, define the $j$ th order differential operators $\mathscr{D}_{j}$ on $\hat{\mathcal{R}}$ by

$$
\mathscr{D}_{j}=\sum_{\boldsymbol{i} \in \mathbb{Z}_{\geq 0}^{j}} \sum_{\boldsymbol{a} \in \mathbb{Q}^{j}}\left(\begin{array}{c}
|\boldsymbol{i}| \\
i_{1}, i_{2}, \ldots, i_{j}
\end{array}\right) Q_{|\boldsymbol{i}|}(|\boldsymbol{a}|) \partial_{\boldsymbol{i}}(\boldsymbol{a}),
$$

where

$$
\partial_{\boldsymbol{i}}(\boldsymbol{a})=\frac{\partial^{j}}{\partial Q_{i_{1}+1}\left(a_{1}\right) \cdots \partial Q_{i_{n}+1}\left(a_{n}\right)} .
$$

From now on we pretend that these operators act on $\Lambda^{*}(N)$, by identifying $\Lambda^{*}(N)$ with a quotient of $\hat{\mathcal{R}}$ via the obvious inclusion map. Note that restricted to $\Lambda^{*}$ the operators $\mathscr{D}_{j}$ are the same as defined in Definition 2.3.8. Similarly, the operators $\mathscr{D}_{j}$ satisfy the following property.

Proposition 7.4.2. For all $j \geq 1$ and $\boldsymbol{a} \in \mathbb{Q}^{n}$ one has

$$
\left\langle\mathscr{D}_{j} W(\boldsymbol{z}+\boldsymbol{a})\right\rangle_{q}=j !\left(\left(z_{1}+a_{1}\right) \mathfrak{d}_{z_{1}}^{j-1}+\ldots+\left(z_{n}+a_{n}\right) \mathfrak{d}_{z_{n}}^{j-1}\right) F_{n}(\boldsymbol{z}+\boldsymbol{a}) .
$$

Therefore, Theorem 7.2.3 now specialises to the following result.

Theorem 7.4.3. Let $N \geq 1$ and $\partial=\mathscr{D}_{1}$ and $\mathscr{D}_{2}: \Lambda^{*}(N) \rightarrow \Lambda^{*}(N)$ be given by Equation 7.4.1. Let the projection $\pi: \Lambda^{*}(N) \rightarrow \Lambda^{*}(N)$ be given by

$$
\pi(f)=\sum_{r \geq 0} \sum_{s=0}^{r}(-1)^{s} \frac{Q_{2}^{r} \partial^{2 r-2 s} \mathscr{D}_{2}^{s}(f)}{2^{r}\left(\ell-r-\frac{3}{2}\right)_{r}(r-s) ! s !},
$$

whenever $f \in \Lambda^{*}(N)$ is homogeneous of weight $\ell$. Then, the subspace $\mathcal{M}(N):=$ $\pi\left(\Lambda^{*}(N)\right)$ of $\Lambda^{*}(N)$ satisfies

(i) $\Lambda^{*}(N)=\mathcal{M}(N) \oplus Q_{2} \Lambda^{*}(N)$;

(ii) $\langle\mathcal{M}(N)\rangle_{q} \subseteq M(\Gamma(N))$;

(iii) $\left\langle Q_{2} \Lambda^{*}(N)\right\rangle_{q} \cap M(\Gamma(N))=\{0\}$. 


\subsection{Third example: double moment functions}

For the algebra of double moments functions the $n$-point functions with respect to the induced product (see Definition 4.4.1) are given by

$$
G_{n}(\boldsymbol{u}, \boldsymbol{v})=\prod_{i}\left(\frac{\Theta\left(u_{i}+v_{i}\right)}{\Theta\left(u_{i}\right) \Theta\left(v_{i}\right)}\right)
$$

(see Theorem 4.8.11 and also Section 6.6). Note that $G_{n}$ is a Jacobi form. Hence, the operators $\mathfrak{d}_{\tau}, \mathfrak{d}_{u}$ and $\mathfrak{d}_{v}$ vanish acting on $G_{n}$, so that $\mathscr{D}$ can taken to be the zero operator. Recall the derivation $\mathfrak{d}$ on $\mathcal{T}$ given by Definition 4.10.1, i.e.,

$$
\mathfrak{d}\left(T_{k, \ell}\right)=k(\ell-1) T_{k-1, \ell-1}-\frac{1}{2} \delta_{k+\ell-2}, \quad \mathfrak{d}(f \odot g)=\mathfrak{d}(f) \odot g+f \odot \mathfrak{d}(g)
$$

for all $f, g \in \mathcal{T}$, and satisfying $\langle\mathfrak{d} f\rangle_{q}=\mathfrak{d}_{\tau}\langle f\rangle_{q}$ for all $f \in \mathcal{T}$.

Theorem 7.5.1. Let the projection $\pi: \mathcal{T} \rightarrow \mathcal{T}$ be given by

$$
\pi(f)=\sum_{r \geq 0} \frac{2^{r}}{r !} \overbrace{T_{1,1} \odot \cdots \odot T_{1,1}}^{r} \odot \mathfrak{d}^{r}(f) .
$$

Then $\mathcal{M}=\pi(\mathcal{T})$ satisfies the following three properties:

(i) $\mathcal{T}=\mathcal{M} \oplus\left(T_{1,1}\right)$, where $\left(T_{1,1}\right)=T_{1,1} \odot \mathcal{T}$;

(ii) $\langle\mathcal{M}\rangle_{q}=M$;

(iii) $\left\langle T_{1,1} \odot \mathcal{T}\right\rangle_{q} \cap M=\{0\}$.

Proof. The statement follows along the same lines as Theorem 7.2.3, by making the following observations:

- Analogous to $\xi_{\ell}(\varphi)$, the functions

$$
\sum_{r \leq|\ell|}(-1)^{r} \sum_{s \leq r} \frac{\mathbb{e}_{2}^{r}\left(\mathcal{Q}^{r-s} g_{\ell}\right)(\varphi)_{s}}{(r-s) !}
$$

are modular forms exactly if $\varphi$ is a quasi-Jacobi form;

- $\left\langle T_{1,1} \odot f\right\rangle_{q}=-2 \mathbb{e}_{2}\langle f\rangle_{q}$ for all $f \in \mathbb{C}^{\mathscr{P}}$;

- The operator $\mathfrak{d}$ coincides with the operator $\mathscr{Q}$.

- Theorem 4.6.1 implies that $\langle\mathcal{T}\rangle_{q}=\widetilde{M}$, from which it follows that equality holds in (ii). 
Remark 7.5.2. In fact, for all $f, g \in \mathcal{T}$ one has

$$
\pi(f \odot g)=\pi(f) \odot \pi(g) .
$$

Hence, $\langle\pi \mathcal{T}\rangle_{q}$ is uniquely determined by $\left\langle\pi\left(T_{2,0}\right)\right\rangle_{q}=\left\langle\pi\left(T_{1,1}\right)\right\rangle_{q}=0$ and

$$
\left\langle\pi\left(T_{k, l}\right)\right\rangle_{q}= \begin{cases}\vartheta^{k-1} G_{l-k+2} & l \geq k \\ \vartheta^{l} G_{k-l} & k \geq l+2\end{cases}
$$

for $T_{k, l} \in \mathcal{T}$ with $k+l \geq 4$, where $\vartheta$ denotes the Serre derivative, defined in Section 4.6. The derivatives of Eisenstein series appearing on the right of this equation, with the case distinction according to the sign of $l+1-k$, should be compared to the Taylor coefficients of $G_{1}(u, v)$ in Theorem 4.8.11 or [Zag91]. Moreover, they are very similar (but here in level 1 and there in level 2, and here with Serre derivatives and there with usual derivatives) to the ones that appeared in [KZ95] to prove the original assertion of Dijkgraaf from which the whole Bloch-Okounkov story arose. 
CHAPTER 8

\section{A Kaneko-Zagier equation for quasi-Jacobi forms}

\subsection{Introduction}

In [Obe18, OP16] Oberdieck and Pandharipande conjectured the existence of quasiJacobi forms $\varphi_{m}$ and $\varphi_{m, n}$ (of explicit weight and index) explaining part of the Gromov-Witten theory of K3 surfaces. So far, these functions have been left indeterminate. The goal of this chapter is simply to give an explicit formula for these functions and study their properties, which is of independent interest. Before we do so, we discuss the Kaneko-Zagier and the problem in algebraic geometry in finite characteristic from which it arises, because the functions $\varphi_{m}$ turn out to be a generalisation of the solutions $f_{k}$ of the Kaneko-Zagier equation.

\section{Supersingular elliptic curves and the Kaneko-Zagier equation}

Let $E$ be an elliptic curve over an algebraically closed field of characteristic $p>0$. If its corresponding group of $p$-torsion points is trivial, such an elliptic curve is called supersingular. This condition only depends on the $j$-invariant of $E$. As there are only finitely many supersingular $j$-invariants, one can construct the supersingular polynomial

$$
\operatorname{ss}_{p}(j)=\prod_{\substack{E / \overline{\mathbb{F}}_{p} \\ E \text { supersingular }}}(j-j(E)) \quad \in \overline{\mathbb{F}}_{p}[j] .
$$

This polynomial is uniquely determined by a modular form, which we explain now. For every $k \in \mathbb{Z}$ the function

$$
f_{k}=\operatorname{Res}_{x=0} \wp^{\prime}(x)^{\frac{k+1}{3}}
$$

This chapter is based on [IOP20], joint work with Georg Oberdieck and Aaron Pixton. 
is a modular form of weight $k$. Surprisingly, this family of modular forms is a family of solutions of the Kaneko-Zagier differential equation [KZ98]

$$
D_{\tau}^{2} f_{k}+4(k+1) G_{2} D_{\tau} f_{k}=2 k(k+1) D_{\tau}\left(G_{2}\right) f_{k} .
$$

Denote by $\Delta=\eta^{24}=\frac{Q^{3}-R^{2}}{1728}$ and $j=\frac{Q^{3}}{\Delta}$ the modular discriminant and modular $j$-invariant respectively (which are the discriminant and $j$-invariant of the complex elliptic curve $\mathbb{C} /(\mathbb{Z} \tau+\mathbb{Z})$ ). Then, $f_{k} / \Delta^{k / 12}$ is holomorphic for $\tau \in \mathfrak{h}$ and transforms like a modular form of weight 0 . If we, for simplicity, assume that $k \equiv 0 \bmod 12$, this function can uniquely be written as a polynomial $\tilde{f}_{k}$ in the modular $j$-invariant. Without this assumption one first multiplies $f_{k}$ by $Q^{2 \delta} R^{\epsilon}$, where $12 \mid k+8 \delta+6 \epsilon$ and $\delta \in\{0,1,2\}$ and $\epsilon \in\{0,1\}$. After dividing the product by an integral power of $\Delta$ such that the result is of weight 0 , it is again a polynomial in the $j$-invariant and $\tilde{f}_{k}$ is defined analogously. (Equivalently, as in [KZ98], one could divide $f_{k}$ by $Q^{\delta} R^{\epsilon}$ and a suitable integral power of $\Delta$. Then, $\tilde{f}_{k}$ divided by the resulting polynomial in $j$ is a polynomial with only zeros at 0 and 1728.) A main result of [KZ98] is that for primes $p>3$ one has

$$
\operatorname{ss}_{p}(j) \equiv c \tilde{f}_{p-1}(j) \quad \bmod p
$$

for some constant $c$ (taken to be \pm 1 in [KZ98] by a suitable normalization of $f_{k}$ ).

Example 8.1.1. Let $p=37$. Recall Ramanujan's notation for the Eisenstein series, as in Example 1.2.5. Up to a constant, $f_{36}$ is then given by

$$
81 Q^{9}+1296 Q^{6} R^{2}+1440 Q^{3} R^{4}+128 R^{6}=\Delta^{3} \tilde{f}_{36}(j)
$$

with

$$
\tilde{f}_{36}(j)=2945 j^{3}+7879680 j^{2}+5446434816 j-660451885056 .
$$

As

$$
\tilde{f}_{36}(j) \equiv 22(j-8)\left(j^{2}-6 j-6\right) \bmod 37,
$$

the supersingular $j$-invariants for $p=37$ are given by $j=8$ and by the roots of $j^{2}-6 j-6$ in $\mathbb{F}_{37^{2}}$.

\section{A Kaneko-Zagier equation for Jacobi forms}

Let $\tau \in \mathfrak{h}, z \in \mathbb{C}, q=e^{2 \pi \mathrm{i} \tau}, p=e^{2 \pi \mathrm{i} z}$ and $D_{\tau}=\frac{1}{2 \pi \mathrm{i}} \frac{d}{d \tau}=q \frac{d}{d q}$. Recall that the normalised Jacobi theta function $\Theta$ is given by $\Theta(\tau, z)=\frac{\theta(\tau, z)}{\theta^{\prime}(\tau, 0)}$ with $\theta$ given by (1.14). Consider the ratio

$$
F(z):=\frac{D_{\tau}^{2} \Theta(z)}{\Theta(z)}=-\sum_{m, r \geq 1} r^{3}\left(p^{m / 2}-p^{-m / 2}\right)^{2} q^{m r},
$$


where, as we often do, we have dropped $\tau$ from the argument.

We define formal series $\varphi_{m} \in \mathbb{Q}\left[p^{ \pm \frac{1}{2}}\right][[q]]$ for all $m \in \mathbb{Z}$ by the differential equation

$$
D_{\tau}^{2} \varphi_{m}=m^{2} F \varphi_{m},
$$

together with the constant term

$$
\varphi_{m}=\left(p^{m / 2}-p^{-m / 2}\right)+O(q) .
$$

Since the constant term of $F$ in $q$ vanishes, the differential equation (8.2) determines the functions $\varphi_{m}$ uniquely from the initial data. By definition, we have $\varphi_{-m}=-\varphi_{m}$.

Our first main result, which is the first part of Theorem $\mathrm{H}$, is the following characterisation of the functions $\varphi_{m}$.

Theorem 8.1.2. Let $m \geq 0$. Then,

$$
\varphi_{m}(z)=\operatorname{Res}_{x=0}\left(\frac{\Theta(x+z)}{\Theta(x)}\right)^{m} .
$$

In particular, $\varphi_{m}$ is a holomorphic quasi-Jacobi form of weight -1 and index $|m| / 2$.

Consider the ratio of theta functions

$$
f(x)=\frac{\Theta(x+z)}{\Theta(x)}
$$

whose appearance in mathematics goes back to work of Eisenstein [Wei99]. Since its inverse has Taylor expansion $1 / f(x)=\Theta(z)^{-1} x+O\left(x^{2}\right)$, the function $1 / f(x)$ can be formally inverted. By Lagrange inversion, Theorem 8.1.2 then precisely says that the inverse series is the generating series of the $\varphi_{m}$ :

$$
y=\frac{1}{f(x)} \quad \Longleftrightarrow \quad x=\sum_{m=1}^{\infty} \frac{\varphi_{m}}{m} y^{m} .
$$

Let us explain the connection of the differential equation (8.2) to the KanekoZagier equation given by (8.1). A direct calculation shows that a function $f_{k}$ is a solution to the Kaneko-Zagier equation if and only if $g_{k+1}=f_{k} / \eta^{2 k+2}$ is a solution of

$$
D_{\tau}^{2} g_{m}=\frac{5}{3} m^{2} G_{4} g_{m} \quad(m=k+1)
$$

We observe that the differential equation (8.2) is a Jacobi-form analogue of the Kaneko-Zagier equation. Even stronger, since (8.2) does not involve derivatives in the elliptic variable we can specialise it to $z=a$ for any $a \in \mathbb{Q}$ and in this way 
obtain an infinite family of Kaneko-Zagier type differential equations with modular solutions for a congruence subgroup (the proof is immediate if one notes that for any $a \in \mathbb{Q}$ the ratio $\frac{\Theta(x+a)}{\Theta(x)}$ is of index 0 as a meromorphic Jacobi form of higher level, hence all Taylor coefficients are modular forms rather than quasimodular forms).

Corollary 8.1.3. For all $a \in \mathbb{Q}$ and $m \in \mathbb{Z}$, the modular form $\varphi_{m}(a)$ is a solution $g_{m}$ of the Kaneko-Zagier type equation

$$
D_{\tau}^{2} g_{m}=m^{2} F(a) g_{m},
$$

where $F(a)$ denotes the Eisenstein series

$$
F(a)=-\sum_{m, r \geq 1} r^{3}\left(\boldsymbol{e}(a)^{m}+\boldsymbol{e}(a)^{-m}-2\right) q^{m r} \quad\left(\boldsymbol{e}(a)=e^{2 \pi \mathrm{i} a}\right) .
$$

The inversion formula (8.5) has the classical analogue [KZ98, Thm. 5(iv)]

$$
x=\sum_{k \geq 1} \frac{f_{k-1}}{k} y^{k} \Longleftrightarrow y=\wp^{\prime}(x)^{-1 / 3},
$$

where the role of $f(x)$ is played by the formal cube root of the derivative $\wp^{\prime}(x)=$ $\frac{d}{d x} \wp(x)$ of the Weierstrass elliptic function $\wp$, and the solutions $f_{k}$ are normalised accordingly.

We refer to Section 8.7 for a general construction of differential equations of Kaneko-Zagier type.

\section{Holomorphic anomaly equations and polynomiality}

Holomorphic anomaly equations for a quasi-Jacobi form $\psi$ are by definition an expression for $\mathfrak{d}_{\tau}^{i} \psi$ and $\mathfrak{d}_{z}^{i} \psi$ for all $i$. As discussed in Chapter $5, \mathfrak{d}_{\tau} \psi$ and $\mathfrak{d}_{z} \psi$ measure the defect of $\psi$ to be honest Jacobi forms. Said differently, holomorphic anomaly equations determine the precise transformation behaviour of $\psi$ under the Jacobi group. In this chapter, all quasi-Jacobi forms are polynomials in $A, \wp, \wp^{\prime}, G_{2}, G_{4}$ and $\Theta$ and we often use $\frac{\partial}{\partial G_{2}}$ and $\frac{\partial}{\partial A}$ instead of $\mathfrak{d}_{\tau}$ and $\mathfrak{d}_{z}$ (see Remark 5.6.3).

Proposition 8.1.4. For all $m \geq 1$ one has

$$
\frac{\partial}{\partial G_{2}} \varphi_{m}=0 \quad \text { and } \quad \frac{\partial}{\partial A} \varphi_{m}=\frac{1}{2} \sum_{\substack{i+j=m \\ i, j \geq 1}} \frac{m^{2}}{i j} \varphi_{i} \varphi_{j}
$$


Whereas the holomorphic anomaly equations determine the transformation of the individual $\varphi_{m}$ 's, we prove that the dependence of $\varphi_{m}$ on $m$ is polynomially. More precisely, we see in Proposition 8.3.1 that

$$
\varphi_{m}=\sum_{k \geq 1} P_{k}(m) z^{k}
$$

where each $P_{k}$ is a polynomial in $m$ of degree at most $k$ with quasimodular forms as coefficients.

\section{A second differential equation}

We are also interested in a second family of functions, defined in terms of the $\varphi_{m}$. Define formal series $\varphi_{m, n} \in \mathbb{Q}\left[p^{ \pm 1 / 2}\right][[q]]$ for all $m, n \in \mathbb{Z}$ by the differential equation

$$
D_{\tau} \varphi_{m, n}=m n \varphi_{m} \varphi_{n} F+\left(D_{\tau} \varphi_{m}\right)\left(D_{\tau} \varphi_{n}\right)
$$

together with the condition that the constant term vanishes: $\varphi_{m, n}=O(q)$. Since $\varphi_{m}$ is odd in $m$, the definition implies the symmetries

$$
\forall m, n: \varphi_{m, n}=\varphi_{n, m}=\varphi_{-m,-n} .
$$

Moreover, $\varphi_{m, 0}=0$ as $\varphi_{0}=0$. Our second main result describes the modular properties of $\varphi_{m, n}$.

Theorem 8.1.5. For all $m, n \in \mathbb{Z}$ the difference

$$
\varphi_{m, n}-|n| \delta_{m+n, 0}
$$

is a quasi-Jacobi form of weight 0 and index $\frac{1}{2}(|m|+|n|)$.

In particular, if we would have normalised $\varphi_{m, n}$ by $\varphi_{m, n}=|n| \delta_{m+n, 0}+O(q)$, the functions $\varphi_{m, n}$ would have been quasi-Jacobi forms for all $m, n$. Hence, this result together with Theorem 8.1.2 is the content of Theorem $\mathrm{H}$. In the rest of this chapter we assume $\varphi_{m, n}=O(q)$ in order not to break the polynomial dependence on $m$ and $n$.

If $m \neq-n$ the proof of Theorem 8.1.5 is easy. Indeed, in this case we have

$$
\varphi_{m, n}=\frac{m}{m+n} \varphi_{m} D_{\tau}\left(\varphi_{n}\right)+\frac{n}{m+n} D_{\tau}\left(\varphi_{m}\right) \varphi_{n}
$$

and since the algebra of quasi-Jacobi forms is closed under differentiation with respect to both $z$ and $\tau$ the result follows from Theorem 8.1.2. It hence remains to 
consider the case $m=-n$. However, since the algebra of quasi-Jacobi forms is not closed under integration, this case is not obvious.

The polynomial dependence of $\varphi_{m}$ on $m$ implies that $\varphi_{m, n}$ depends polynomially on $m, n$ as well. Hence we are allowed to take the limit of the formula (8.7). The result is

$$
\varphi_{n,-n}=D_{\tau}\left(\varphi_{n}\right) \varphi_{-n}+n\left(D_{\tau}\left(\varphi_{-n}^{\prime}\right) \varphi_{n}-\varphi_{-n}^{\prime} D_{\tau} \varphi_{n}\right),
$$

where $\varphi_{u}^{\prime}$ is the formal derivative of $\varphi_{u}$ with respect to $u$. But, by inspection the function $\varphi_{n}^{\prime}$ is usually not a quasi-Jacobi form and hence from this point it is still unclear why $\varphi_{n,-n}$ should be quasi-Jacobi. Instead our proof of Theorem 8.1.5 relies on a subtle interplay between holomorphic anomaly equations and the aforementioned polynomiality.

As another indirect consequence of the proof of Theorem 8.1.5 we obtain a third, recursive characterisation of the function $\varphi_{m}$.

Proposition 8.1.6. For all $m, n \geq 1$ we have

$$
\varphi_{m+n}=\frac{1}{m} D_{z}\left(\varphi_{m}\right) \varphi_{n}+\frac{1}{n} \varphi_{m} D_{z}\left(\varphi_{n}\right)+\sum_{i+j=m} \frac{1}{i} \varphi_{i, n} \varphi_{j}+\sum_{i+j=n} \frac{1}{i} \varphi_{i, m} \varphi_{j} .
$$

Finally, we mention a conjectural relationship between the geometry of K3 surfaces and the functions $\varphi_{m}$ and $\varphi_{m, n}$.

Conjecture 8.1.7. The functions $\varphi_{m}$ and $\varphi_{m, n}$ as defined above are the functions appearing in the conjectures in [Obe18, OP16].

\subsection{The solutions of the differential equation}

In this section we study the function $\varphi_{m}$ defined by the differential equation (8.2) and the constant term (8.3). We first prove the evaluation (8.4) which immediately implies that $\varphi_{m}$ is a quasi-Jacobi form. We then study the Fourier expansion of $\varphi_{m}$, discuss the dependence of $\varphi_{m}$ on the parameter $m$, and derive a holomorphic anomaly equation. 


\section{Proof of Theorem 8.1.2}

To prove the theorem, we define the functions $\varphi_{m}$ for $m \geq 0$ by (8.4). Then, we need to check that these function satisfy the differential equations (8.2) and have the right constant term (8.3), in other words, that these functions coincide with the uniquely defined functions at the beginning of this chapter. Checking the constant term is straightforward, see for example (8.9) below. To check the differential equation we form the generating series $g(y)=\sum_{m \geq 1} y^{m} \varphi_{m} / m$. Let also $D_{y}=y \frac{d}{d y}$. The differential equation (8.2) is then equivalent to

$$
D_{\tau}^{2} g(y)=F(z) D_{y}^{2} g(y) .
$$

Consider the function $f(x)=\frac{\Theta(x+z)}{\Theta(x)}$ and apply the variable change

$$
y=\frac{1}{f(x)} \Longleftrightarrow x=g(y),
$$

where we have used Lagrange inversion to identify the inverse of $1 / f$ with the generating series $g(y)$. Let $f^{\prime}(x):=D_{x} f:=\frac{d}{d x} f(x)$. By differentiating $f(g(y))=y$ and applying the chain rule we find the transformations

$$
\begin{gathered}
D_{y} g(y)=-\frac{f}{f^{\prime}}, \quad D_{\tau} g(y)=-\frac{D_{\tau} f}{f^{\prime}}, \quad D_{y}^{2} g(y)=-\frac{f}{f^{\prime}} \cdot \frac{f^{\prime \prime} f-\left(f^{\prime}\right)^{2}}{\left(f^{\prime}\right)^{2}}, \\
D_{\tau}^{2} g(y)=-\frac{1}{\left(f^{\prime}\right)^{3}}\left(D_{\tau}^{2}(f)\left(f^{\prime}\right)^{2}-2 f^{\prime} D_{\tau}(f) D_{\tau}\left(f^{\prime}\right)+f^{\prime \prime} D_{\tau}(f)^{2}\right) .
\end{gathered}
$$

Applying these and changing variables, the differential equation (8.8) becomes

$$
\begin{gathered}
D_{x}(f)^{2} D_{\tau}^{2}(f)-2 D_{x}(f) D_{x} D_{\tau}(f) D_{\tau}(f)+D_{x}^{2}(f) D_{\tau}(f)^{2}= \\
F(z) D_{x}^{2} \log (f) f^{3} .
\end{gathered}
$$

The functions $\Theta(x+z)$ and $\Theta(x)$ are Jacobi forms of rank 2 in the elliptic variables $(x, z)$ of index $Q(x, z)=\frac{1}{2}(x+z)^{2}$ and $Q(x, z)=\frac{1}{2} x^{2}$ respectively. (Or, in matrix notation, of index $\frac{1}{2}\left(\begin{array}{ll}1 & 1 \\ 1 & 1\end{array}\right)$ and $\frac{1}{2}\left(\begin{array}{ll}1 & 0 \\ 0 & 0\end{array}\right)$ respectively.) Hence, $f(x)$ is a meromorphic Jacobi form of weight 0 and index $Q(x, z)=\frac{1}{2}(2 x+z) z$. We need to show that the following function vanishes:

$$
\begin{aligned}
\mathcal{F}(x, z)=D_{x}(f)^{2} D_{\tau}^{2}(f)-2 D_{x}(f) D_{x} D_{\tau}(f) D_{\tau}(f) & +D_{x}^{2}(f) D_{\tau}(f)^{2}+ \\
& -F(z) D_{x}^{2} \log (f) f^{3} .
\end{aligned}
$$

As a polynomial in the derivatives of $f$, the function $\mathcal{F}$ is a rank 2 quasi-Jacobi form of weight 6 and index $Q(x, z)=\frac{3}{2}(2 x+z) z$. Using the commutation relations in Remark 5.7.3 a direct check shows

$$
\mathfrak{d}_{\tau} \mathcal{F}=\mathfrak{d}_{x} \mathcal{F}=0 .
$$


In particular, $\mathcal{F} / f^{3}$ has index $Q(x, z)=0$, i.e., $\mathcal{F} / f^{3}$ is doubly periodic. Moreover, by considering the Taylor expansion one checks (e.g., using a computer ${ }^{1}$ ) that $\mathcal{F}$ is holomorphic at $x=0$ and vanishes to order 3 at $x=-z$ (use the variable change $\tilde{x}=$ $x+z$ ). We conclude that the ratio $\mathcal{F} / f^{3}$ is doubly periodic and holomorphic in $x$, so constant in $x$. The constant is a quasi-Jacobi form in $z$ and is easily checked to vanish. This shows that the differential equation is satisfied. The claim that the $\varphi_{m}$ are quasi-Jacobi forms of the specified weight follows from Lemma 8.2.1 below.

Define the operator on the algebra of quasi-Jacobi forms by

$$
\hat{D}_{z}=D_{z}+2 G_{2} \frac{\partial}{\partial A} .
$$

We conclude the following structure result.

Lemma 8.2.1. For every $m \geq 0$ there exist modular forms $h_{k} \in M_{m-k-1}$ such that

$$
\varphi_{m}(z)=\sum_{k=0}^{m-1} h_{k} \hat{D}_{z}^{k}\left(\Theta(z)^{m}\right) .
$$

Hence every $\varphi_{m}$ is a quasi-Jacobi form of weight -1 and index $\frac{|m|}{2}$, and $\frac{\partial}{\partial G_{2}} \varphi_{m}=0$.

Proof. For any power series $f(z)$ we have

$$
e^{D_{z} x} f(z)=f(x+z) .
$$

Moreover, the Baker-Campbell-Hausdorff formula and the relations in Remark 5.7.3 yield

$$
e^{D_{z} x} e^{2 G_{2} \frac{\partial}{\partial A} x}=e^{x \hat{D}_{z}-2 x^{2} G_{2} I}=e^{-2 x^{2} G_{2} I} e^{x \hat{D}_{z}},
$$

where $I$ denotes the operator acting diagonally on Jacobi forms of rank 1 by multiplication by the index. We find that

$$
\begin{aligned}
\frac{\Theta(x+z)^{m}}{\Theta(x)^{m}} & =\Theta(x)^{-m} e^{D_{z} x}\left(\Theta(z)^{m}\right) \\
& =\Theta(x)^{-m} e^{D_{z} x} e^{2 G_{2} \frac{\partial}{\partial A} x}\left(\Theta(z)^{m}\right) \\
& =\Theta(x)^{-m} e^{-m x^{2} G_{2}} e^{x \hat{D}_{z}}\left(\Theta(z)^{m}\right) \\
& =x^{-m} \exp \left(2 m \sum_{k \geq 4} G_{k} \frac{x^{k}}{k !}\right) e^{x \hat{D}_{z}}\left(\Theta(z)^{m}\right),
\end{aligned}
$$

\footnotetext{
${ }^{1}$ The code for this computation as well as a parallel computation in the proof of Proposition 8.5.5 can be found on the web page of Georg Oberdieck (https://www .math.uni-bonn.de/ georgo/ note/KZ_code.py). It also contains functions which express the $\varphi_{m}, \varphi_{m n}$ in terms of $A, \wp, \wp^{\prime}$, $G_{2}, G_{4}$ and $\Theta$.
} 
where we used (5.2) in the last step. Taking the coefficient of $x^{-1}$ yields the first claim. The second claim follows from the commutation relation $\left[\frac{\partial}{\partial G_{2}}, \hat{D}_{z}\right]=0$.

Remark 8.2.2. For all $m \geq 0$ we have

$$
\varphi_{-m}(z)=\operatorname{Res}_{x=-z}\left(\frac{\Theta(x+z)}{\Theta(x)}\right)^{-m} .
$$

Indeed, after the variable change $x^{\prime}=-(x+z)$ the right-hand side becomes

$$
-\operatorname{Res}_{x^{\prime}=0}\left(\frac{\Theta\left(-x^{\prime}-z\right)}{\Theta\left(-x^{\prime}\right)}\right)^{m}=-\varphi_{m}(z) .
$$

\subsection{Three expansions of the solutions}

We give three different formulas for $\varphi_{m}$, that is we compute the Fourier expansion, and two types of Taylor expansions in the parameter $m$.

\section{Fourier expansion}

Write

$$
f_{m}(x, z)=\left(\frac{\Theta(x+z)}{\Theta(x)}\right)^{m} .
$$

For a contour $\gamma$ around $z=0$ and not around any other lattice point $z \in \mathbb{Z} \tau+\mathbb{Z}$ we have

$$
\varphi_{m}(z)=\operatorname{Res}_{x=0} f_{m}(x, z)=\frac{1}{2 \pi \mathrm{i}} \oint_{\gamma} f_{m}(x, z) \mathrm{d} x .
$$

Let $a=-\frac{1}{2}-\frac{1}{2} \tau$. Choose the parallelogram with corners $a, a+\tau, a+\tau+1$ and $a+1$ for $\gamma$ (which is the boundary of a fundamental domain for the lattice). By the elliptic transformations $f_{m}(x+1, z)=f_{m}(x, z)$ and $f_{m}(x+\tau, z)=p^{-m} f_{m}(x, z)$ one gets

$$
\varphi_{m}(z)=\frac{1}{2 \pi \mathrm{i}}\left(1-p^{-m}\right) \int_{0}^{1} f_{m}(a+t, z) \mathrm{d} t=\frac{1}{2 \pi \mathrm{i}}\left(1-p^{-m}\right) \text { Coeff }_{\sigma^{0}} f_{m}(x, z),
$$

where $\sigma=e^{2 \pi \mathrm{i} x}$ is the Fourier variable associated to $x$ and the Fourier expansion is taken for $x$ in the strip $\operatorname{Im}(\tau)<\operatorname{Im}(x)<0$. (See also [OP18, App. A] for a similar argument.)

By the Jacobi triple product

$$
f_{1}(x, z)=s \prod_{n=1}^{\infty} \frac{\left(1-q^{n} \sigma p\right)\left(1-q^{n} \sigma^{-1} p^{-1}\right)}{\left(1-q^{n} \sigma\right)\left(1-q^{n} \sigma^{-1}\right)},
$$


where we have written $s=e^{\pi \mathrm{i} z}$, so $p=s^{2}$. Hence,

$$
\log f_{m}(x, z)=\frac{1}{2} z-\sum_{n=1}^{\infty} \sum_{r \neq 0} \frac{m}{r} \sigma^{r}\left(p^{r}-1\right) q^{m r} .
$$

Exponentiating again, we find

$$
\begin{aligned}
\varphi_{m} & =\left(p^{m / 2}-p^{-m / 2}\right) \operatorname{Coeff}_{\sigma^{0}} \exp \left(\sum_{r \neq 0} \frac{m}{r} \sigma^{r} \frac{1-p^{k}}{1-q^{r}}\right) \\
& =\left(p^{m / 2}-p^{-m / 2}\right) \sum_{|a|=0}\left(\prod_{i} \frac{1-p^{a_{i}}}{1-q^{a_{i}}}\right) \frac{m^{l(a)}}{\mathfrak{z}(a)},
\end{aligned}
$$

where the sum in the second equation is over all generalised partitions (allowing both positive and negative parts) with non-zero parts summing up to 0 . Moreover, if we write $a$ in exponential notation $a=\left(i^{a_{i}}\right)_{i \in \mathbb{Z} \backslash\{0\}}$ then $\mathfrak{z}(a)=\prod_{i} i^{a_{i}} a_{i}$ ! is the standard automorphism factor (4.44). The first Fourier coefficients of $\varphi_{m}$ are

$$
\varphi_{m}=\left(s^{m}-s^{-m}\right)\left(1-m^{2}\left(s-s^{-1}\right)^{2} q\right)+O\left(q^{2}\right) .
$$

\section{The solution $\varphi_{m}$ as a function of $m$}

Consider $\varphi_{m}$ as a function of $m$ viewed as a (formal) variable. To distinguish with the case $m \in \mathbb{Z}$ we will replace $m$ by a variable $u$.

Consider the expansion

$$
F(z)=\sum_{k \geq 1} F_{k}(z) q^{k}, \quad F_{k}(z)=-\sum_{d \mid k}\left(\frac{k}{d}\right)^{3}\left(s^{d}-s^{-d}\right)^{2},
$$

where as before we have used $s=e^{\pi \mathrm{i} z}$, so $p=s^{2}$. Then by an immediate check the differential equation (8.2) for $\varphi_{m}$ is equivalent to the following formula:

$$
\begin{aligned}
\varphi_{u}= & \left(p^{u / 2}-p^{-u / 2}\right) . \\
& \left(1+\sum_{r \geq 1} \sum_{k_{1}, \ldots, k_{r} \geq 1} \frac{F_{k_{1}}(z) F_{k_{2}}(z) \cdots F_{k_{r}}(z)}{k_{1}^{2}\left(k_{1}+k_{2}\right)^{2} \cdots\left(k_{1}+\ldots+k_{r}\right)^{2}} q^{k_{1}+\ldots+k_{r}} u^{2 r}\right) .
\end{aligned}
$$

Second we can use the Fourier expansion of the $\varphi_{m}$ given by (8.9). We see that Theorem 8.1.2 is equivalent to the following non-trivial identity:

$$
\begin{aligned}
& \sum_{|a|=0}\left(\prod_{i} \frac{1-p^{a_{i}}}{1-q^{a_{i}}}\right) \frac{u^{l(a)}}{\mathfrak{z}(a)}= \\
& 1+\sum_{\substack{r \geq 1 \\
k_{1}, \ldots, k_{r} \geq 1}} \frac{F_{k_{1}}(z) F_{k_{2}}(z) \cdots F_{k_{r}}(z)}{k_{1}^{2}\left(k_{1}+k_{2}\right)^{2} \cdots\left(k_{1}+\ldots+k_{r}\right)^{2}} q^{k_{1}+\ldots+k_{r}} u^{2 r} .
\end{aligned}
$$


For the third formula, we use a Taylor expansion in $u$. For positive integers $u$ one can write the solution

$$
\operatorname{Coeff}_{x^{-1}}\left(\frac{\Theta(x+z)}{\Theta(x)}\right)^{u}
$$

as

$$
\begin{aligned}
\varphi_{u}(z) & =\operatorname{Coeff}_{x^{-1}} \frac{(x+z)^{u}}{x^{u}} \exp \left(2 u \sum_{k \geq 2} G_{k} \frac{x^{k}-(x+z)^{k}}{k !}\right) \\
& =\operatorname{Coeff}_{x^{-1}} \sum_{\ell=1}^{\infty}\left(\begin{array}{l}
u \\
\ell
\end{array}\right)\left(\frac{z}{x}\right)^{\ell} \exp \left(2 u \sum_{k \geq 2} G_{k} \frac{x^{k}-(x+z)^{k}}{k !}\right) .
\end{aligned}
$$

The latter expression makes sense as an element of $\mathbb{C}[[z]]$ for all $u \in \mathbb{C}$. For example, the first terms read

$$
\varphi_{u}(z)=u z-G_{2} u^{3} z^{3}+\left(\left(\frac{1}{3} G_{2}^{2}-\frac{1}{72} G_{4}\right) u^{5}+\left(\frac{1}{6} G_{2}^{2}-\frac{5}{72} G_{4}\right) u^{3}\right) z^{5}+O\left(z^{7}\right) .
$$

The expansion (8.10) yields the following important structure result.

Proposition 8.3.1. For every $k \geq 1$ there exist odd polynomials $P_{k}(u)$ of degree $\leq k$ with coefficients in $\widetilde{M}_{k-1}$ such that for all $m \in \mathbb{Z}$

$$
\varphi_{m}(z)=\sum_{\text {odd } k \geq 1} P_{k}(m) z^{k}
$$

Moreover, $P_{1}(u)=u$ and if $k \geq 2$, then $u^{3} \mid P_{k}(u)$.

We find the following language convenient: we say that a set of power series $f_{m}(z) \in R[[z]], m \in \mathbb{Z}$ for some coefficient ring $R$ is polynomial in $m$ if there exist polynomials $P_{k}(u) \in R[u]$ such that

$$
\forall m \in \mathbb{Z}: f_{m}(z)=\sum_{k \geq 0} P_{k}(m) z^{k} .
$$

In the above result $R$ is ring of quasi-modular forms $\widetilde{M}$ and $\varphi_{m}$ is polynomial in $m$.

\subsection{Holomorphic anomaly equations}

The holomorphic anomaly equation $\frac{\partial}{\partial G_{2}} \varphi_{m}=0$ was proven in Lemma 8.2.1. The following result proves Proposition 8.1.4, by determining the holomorphic anomaly of $\varphi_{m}$ with respect to the elliptic variable. 
Proposition 8.4.1. For all $m \geq 1$ one has

$$
\frac{\partial}{\partial A} \varphi_{m}=\frac{1}{2} \sum_{\substack{i+j=m \\ i, j \geq 1}} \frac{m^{2}}{i j} \varphi_{i} \varphi_{j} .
$$

Before we prove this proposition, note that it follows that every $z^{k}$ coefficient of $\frac{\partial}{\partial A} \varphi_{m}$ is polynomial in $m$ in the range $m \geq 0$. Even so, this coefficient is polynomial in $m$ in the range $m \leq 0$. However, these two polynomials do not always agree, which is made precise in the following result:

Corollary 8.4.2. The difference

$$
\varphi_{m}^{A}=\frac{\partial}{\partial A} \varphi_{m}-m z \varphi_{m} \delta_{m<0}
$$

depends polynomially on $m$, i.e., there exist polynomials $Q_{k}(u)$ of degree $\leq k+1$ with coefficients in $\widetilde{M}_{k-2}$ such that $\varphi_{m}^{A}=\sum_{k \geq 2} z^{k} Q_{k}(m)$. Moreover, $u^{2} \mid Q_{k}$ for all $k$.

Proof of Corollary 8.4.2. We first rewrite (8.11) as

$$
\frac{\partial}{\partial A} \varphi_{m}=m \sum_{j=1}^{m-1} \varphi_{j} \frac{\varphi_{m-j}}{m-j} .
$$

Hence for all $m \geq 0$ we have $\frac{\partial}{\partial A} \varphi_{m}=\sum_{n} Q_{n}(m) z^{n}$ where the polynomials $Q_{n}$ are determined by

$$
Q_{n}(m)=m \sum_{\substack{k+\ell=n \\ k, \ell \geq 1}} \sum_{j=1}^{m-1} P_{k}(j) \frac{P_{\ell}(m-j)}{m-j}
$$

for all $m \geq 0$. Here the $P_{i}$ are the polynomials of Proposition 8.3.1.

For all $m>0$ by Lemma 8.5.2 we have

$$
\begin{aligned}
Q_{n}(-m) & =-\left.(-m) \sum_{\substack{k+\ell=n \\
k, \ell \geq 1}} \sum_{j=1}^{m} P_{k}(-j)\left(\frac{P_{\ell}(u)}{u}\right)\right|_{u=-m+j} \\
& =-m \sum_{\substack{k+\ell=n \\
k, \ell \geq 1}} \sum_{j=1}^{m-1} P_{k}(j) \frac{P_{\ell}(-m+j)}{-m+j}-m P_{n-1}(m),
\end{aligned}
$$


where we used the second part of Proposition 8.3.1 for the last equality. Summing up we obtain as desired

$$
\varphi_{-m}^{A}=\sum_{n} z^{n} Q_{n}(-m)=-\frac{\partial}{\partial A} \varphi_{m}-m z \varphi_{m} .
$$

First proof of Proposition 8.1.4. As in the proof of Theorem 8.1.2 consider the generating series

$$
g(y)=\sum_{m \geq 1} \frac{\varphi_{m}}{m} y^{m}
$$

and let $D_{y}=y \frac{d}{d y}$. We need to prove the equality

$$
\frac{\partial}{\partial A(z)} g(y)=g(y) D_{y} g(y)
$$

Let $f(x)=\frac{\Theta(x+z)}{\Theta(x)}$ so that $f(g(y))=\frac{1}{y}$. Then by $\left[\frac{\partial}{\partial A}, D_{z}\right]=2 I$ we have

$$
\frac{\partial}{\partial A(z)} f(x)=\frac{\partial}{\partial A(z)} \frac{e^{D_{z} x} \Theta(z)}{\Theta(x)}=\frac{\left[\frac{\partial}{\partial A(z)}, D_{z}\right] x e^{D_{z} x} \Theta(z)}{\Theta(x)}=x f(x) .
$$

Applying $\frac{\partial}{\partial A}$ to $f(g(y))=1 / y$ we get

$$
\frac{\partial}{\partial A} f(g(y))+D_{x} f(g(y)) \frac{\partial}{\partial A} g(y)=0
$$

and hence

$$
\frac{\partial}{\partial A(z)} g(y)=-\frac{g(y)}{y \cdot\left(D_{x} f\right)}
$$

Since we also have

$$
D_{y}(f(g(y)))=D_{x} f(g(y)) D_{y} g(y)=-\frac{1}{y},
$$

and hence

$$
\frac{1}{D_{x} f}=-y D_{y} g(y)
$$

the claim follows.

We give a more direct proof of Proposition 8.1.4 using the following combinatorial lemma whose proof follows directly from Lagrange inversion. 
Lemma 8.4.3. Let $f(x)$ be a power series and $k \in \mathbb{N}$. Then for all $m \geq 1$ we have

$$
\frac{1}{m}\left[f(x)^{m}\right]_{x^{m-k}}=\frac{1}{k} \sum_{n_{1}+\ldots+n_{k}=m} \prod_{i=1}^{k} \frac{1}{n_{i}}\left[f(x)^{n_{i}}\right]_{x^{n_{i}-1}},
$$

where we write $[-]_{x^{m}}$ for taking the coefficient of $x^{m}$.

Second proof of Proposition 8.1.4. Observe that by (8.12) we have

$$
\frac{\partial}{\partial A}\left[\left(\frac{\Theta(x+z)}{\Theta(x)}\right)^{m}\right]_{x^{-1}}=m\left[\left(\frac{\Theta(x+z)}{\Theta(x)}\right)^{m}\right]_{x^{-2}} .
$$

Applying Lemma 8.4.3 with $k=2$ and $f=x \frac{\Theta(x+z)}{\Theta(x)}$ yields the desired result.

\subsection{A second differential equation}

Recall the two defining properties of the series $\varphi_{m, n}$ :

- the differential equation: $D_{\tau} \varphi_{m, n}=m n \varphi_{m} \varphi_{n} F+\left(D_{\tau} \varphi_{m}\right)\left(D_{\tau} \varphi_{n}\right)$;

- the vanishing of the constant term: $\varphi_{m, n}=O(q)$.

The goal of this section is to first prove that $\varphi_{m, n}$ are quasi-Jacobi forms (Theorem 8.1.5).

\section{Polynomiality}

We first recall the following.

Proposition 8.5.1. If $m \neq-n$ then we have

$$
\varphi_{m, n}=\frac{m}{m+n} \varphi_{m} D_{\tau}\left(\varphi_{n}\right)+\frac{n}{m+n} D_{\tau}\left(\varphi_{m}\right) \varphi_{n} .
$$

Proof. The differential equation follows from the defining differential equation (8.2) satisfied by $\varphi_{m}$. The vanishing of the constant term is observed directly.

By definition and the polynomiality of $\varphi_{m}$ the series $\varphi_{m, n}$ is a power series in $z$ and $q$ with coefficients which are polynomials in $m$ and $n$. In order to strengthen this statement, the following lemma is useful. 
Lemma 8.5.2. Let $f(u, v)$ be a polynomial in variables $u, v$ and let $F(u)$ be the unique polynomial such that $\forall n \geq 1: F(n)=\sum_{j=0}^{n-1} f(j, n-j)$. Then

$$
F(-n)=-\sum_{j=1}^{n} f(-j,-n+j) .
$$

Proof. For all $m \in \mathbb{Z}, n>0$ define

$$
G(m, n)=\sum_{j=0}^{n-1} f(j, m-j) .
$$

This agrees with a unique polynomial $P(m, n)$. Now extend $G$ to all $m, n \in \mathbb{Z}$ by setting $G(m, 0)=0$ and

$$
G(m, n)=-\sum_{j=1}^{-n} G(-j, m+j)
$$

for all $m \in \mathbb{Z}, n<0$. We then have that $G(m, n+1)-G(m, n)=f(n, m-n)$ is a polynomial for all $m, n$. But $P(m, n+1)-P(m, n)$ is also a polynomial. The two polynomials agree for $n>0$, so they agree for all $n$; since $G$ and $P$ also agree for $n>0$, this means that they must also agree for all $n$.

The lemma now follows, since it is just saying that

$$
F(-n)=P(-n,-n)=G(-n,-n) .
$$

We now use Proposition 8.5.1 to prove a stronger statement.

Proposition 8.5.3. There exist polynomials $P_{r}(u, v)$ of degree at most $r$ in the variables $u, v$ with as coefficients quasi-modular forms of weight $r$, such that for all $m, n \in \mathbb{Z}$

$$
\varphi_{m, n}=\sum_{r>0} z^{r} P_{r}(m, n)
$$

Moreover, the polynomials $P_{r}(u, v)$ are divisible by both $u^{2}$ and $v^{2}$.

Proof. By the defining differential equation (8.6) and the polynomiality of $\varphi_{m}$ there exist polynomials $P_{a, r}(u, v)$ of degree $r+2$ with rational coefficients such that

$$
\varphi_{m, n}(z)=\sum_{r>0} z^{r} \sum_{a \geq 1} q^{a} P_{a, r}(m, n)
$$

for all $m, n \in \mathbb{Z}$. Here we have $r>0$ since $\varphi_{m}(0)=0$ for all $m$. 
On the other hand by Proposition 8.5.1 for all $m, n \in \mathbb{Z}$ with $m \neq-n$ we have

$$
\varphi_{m, n}(z)=\sum_{r>0} z^{r} \frac{1}{m+n} \sum_{k+\ell=r}\left(n D_{\tau}\left(P_{k}(m)\right) P_{\ell}(n)+m P_{k}(m) D_{\tau}\left(P_{\ell}(n)\right)\right)
$$

where $P_{k}(u)$ are the polynomials of Proposition 8.3.1. Since the inner sum vanishes when setting $m=-n$ and it is polynomial of degree at most $r+1$ in $m, n$, there exists a polynomial $P_{r}(u, v)$ of degree at most $r$ with coefficients in $\widetilde{M}_{r}$ such that

$$
\varphi_{m, n}(z)=\sum_{r>0} z^{r} P_{r}(m, n)
$$

whenever $m \neq-n$.

The equality of polynomials

$$
\sum_{a \geq 1} q^{a} P_{a, r}(u, v)=P_{r}(u, v)
$$

holds after evaluating $(u, v)$ at $(m, n)$ for all integers $m \neq-n$. Hence the equality holds as an equality of polynomials.

The last statement follows since $n D_{\tau}\left(P_{k}(m)\right) P_{\ell}(n)+m P_{k}(m) D_{\tau}\left(P_{\ell}(n)\right)$ is divisible by both $m^{2}$ and $n^{2}$, hence the same holds for the term obtained by dividing by $m+n$.

Example 8.5.4. The first terms in the Fourier and Taylor expansions of $\varphi_{m, n}$ are

$$
\varphi_{m, n}=-m n\left(s^{m}-s^{-m}\right)\left(s^{n}-s^{-n}\right)\left(s-s^{-1}\right)^{2} q+O\left(q^{2}\right)
$$

where $s=e^{\pi \mathrm{i} z}$, and

$$
\begin{aligned}
\varphi_{u, v}(z)=( & \left.\left(2 G_{2}^{2}-\frac{5}{6} G_{4}\right) u^{2} v^{2}\right) z^{4}+ \\
+ & \left(-\frac{4}{3} G_{2}^{3}+\frac{2}{3} G_{2} G_{4}-\frac{7}{720} G_{6}\right)\left(u^{4} v^{2}+u^{2} v^{4}\right)+ \\
& +\left(-\frac{2}{3} G_{2}^{3}+\frac{1}{6} G_{2} G_{4}+\frac{7}{720} G_{6}\right) u^{3} v^{3}+ \\
& \left.+\left(-\frac{2}{3} G_{2}^{3}+\frac{5}{6} G_{2} G_{4}-\frac{7}{144} G_{6}\right) u^{2} v^{2}\right) z^{6}+O\left(z^{7}\right) .
\end{aligned}
$$

\section{Holomorphic anomaly equations}

From Proposition 8.5.1 we can deduce for all $m \neq-n$ the following anomaly equation:

$$
\begin{aligned}
\frac{\partial}{\partial A} \varphi_{m, n}= & \frac{n}{m+n}\left(D_{z}\left(\varphi_{m}\right) \varphi_{n}+D_{\tau}\left(\frac{\partial}{\partial A} \varphi_{m}\right) \varphi_{n}+D_{\tau}\left(\varphi_{m}\right) \frac{\partial}{\partial A} \varphi_{n}\right)+ \\
& \frac{m}{m+n}\left(\varphi_{m} D_{z}\left(\varphi_{n}\right)+\left(\frac{\partial}{\partial A} \varphi_{m}\right) D_{\tau}\left(\varphi_{n}\right)+\varphi_{m} D_{\tau} \frac{\partial}{\partial A} \varphi_{n}\right) .
\end{aligned}
$$


By the anomaly equation for $\varphi_{m}$ this gives an expression for $\frac{\partial}{\partial A} \varphi_{m, n}$ whenever $m \neq-n$.

In case $m, n>0$ we can find a more efficient equation:

Proposition 8.5.5. For all $m, n>0$,

$$
\frac{\partial}{\partial A} \varphi_{m, n}=\frac{m n}{m+n} \varphi_{m+n}+\sum_{j=1}^{m-1} \frac{m}{j} \varphi_{m-j, n} \varphi_{j}+\sum_{j=1}^{n-1} \frac{n}{j} \varphi_{m, n-j} \varphi_{j}
$$

Proof of Proposition 8.5.5 and Proposition 8.1.6. We prove that the right-hand side in (8.14) is equal to the right-hand side in (8.13). By the anomaly equation for $\varphi_{m}$ and comparing terms it is equivalent to prove the following equation for all $m, n \geq 1$ (which is the statement of Proposition 8.1.6):

$\varphi_{m+n}=\frac{1}{m} D_{z}\left(\varphi_{m}\right) \varphi_{n}+\frac{1}{n} \varphi_{m} D_{z}\left(\varphi_{n}\right)+\sum_{i+j=m} \frac{1}{i} \varphi_{i, n} \varphi_{j}+\sum_{i+j=n} \frac{1}{i} \varphi_{i, m} \varphi_{j}$.

We multiply both sides with $x^{m} y^{n}$ and sum over all $m, n \geq 1$. Write $g(x)=$ $\sum_{m \geq 1} x^{m} \varphi_{m} / m$ and

$$
h(x, y)=D_{x} g(x) \cdot D_{\tau} g(y)+D_{\tau} g(x) \cdot D_{y} g(y) .
$$

Then, the equation becomes

$$
\begin{aligned}
\frac{y D_{x} g(x)-x D_{y} g(y)}{x-y}= & D_{z} g(x) \cdot D_{y} g(y)+D_{x} g(x) \cdot D_{z} g(y)+ \\
& +\left(\left(D_{x}+D_{y}\right)^{-1} D_{y} h(x, y)\right) D_{x} g(x)+ \\
& +\left(\left(D_{x}+D_{y}\right)^{-1} D_{x} h(x, y)\right) D_{y} g(y)
\end{aligned}
$$

where $\left(D_{x}+D_{y}\right)^{-1}$ acts term-wise by multiplying the coefficient of $x^{m} y^{n}$ by the factor $(m+n)^{-1}$ (this is well defined since both $m, n$ are positive for all non-zero coefficients) and we have used

$$
\left(D_{x}+D_{y}\right) \sum_{m, n \geq 1} \frac{\varphi_{m, n}}{m} x^{m} y^{n}=D_{y} h(x, y) .
$$

Rewriting $D_{y}=\left(D_{x}+D_{y}\right)-D_{x}$ we have

$$
\left(D_{x}+D_{y}\right)^{-1} D_{y} h=h-\left(D_{x}+D_{y}\right)^{-1} D_{x} h .
$$


Inserting this the $\left(D_{x}+D_{y}\right)^{-1}$ term factors out and we obtain that (8.16) is equivalent to

$$
\begin{aligned}
D_{x} h=\left(D_{x}+D_{y}\right)\left(\frac{1}{D_{y} g(y)-D_{x} g(x)} .\right. \\
\left.\quad\left(\frac{y D_{x} g(x)-x D_{y} g(y)}{x-y}-D_{z} g(x) \cdot D_{y} g(y)+D_{x} g(x) \cdot D_{z} g(y)\right)\right) .
\end{aligned}
$$

Expanding and using that $\left(D_{x}+D_{y}\right)(y /(x-y))=0$ this is equivalent to the vanishing of

$$
\begin{aligned}
& \left(D_{x}^{2} g(x) D_{y} g(y)-D_{x} g(x) D_{y}^{2} g(y)\right)\left(1+D_{z} g(x)+D_{z} g(y)+h\right)+ \\
& \left(D_{y} g(y)-D_{x} g(x)\right)\left(D_{z}\left(D_{x} g(x) D_{y} g(y)\right)+D_{x} g(x) D_{y}(h)+D_{y} g(y) D_{x} h\right) .
\end{aligned}
$$

We consider again the function $f(x)=\frac{\Theta(x+z)}{\Theta(x)}$ and apply the variable change

$$
x=\frac{1}{f(\tilde{x})}, y=\frac{1}{f(\tilde{y})} \quad \Longleftrightarrow \quad \tilde{x}=g(x), \tilde{y}=g(y) .
$$

Let us denote $f^{\prime}(x)=\frac{d}{d x} f(x)$. We then have the transformations

$$
\begin{aligned}
D_{x} g(x) & =-\frac{f}{f^{\prime}}, & D_{x}^{2} g(x) & =-\frac{f}{f^{\prime}} \cdot \frac{f^{\prime \prime} f-\left(f^{\prime}\right)^{2}}{\left(f^{\prime}\right)^{2}}, \\
D_{z} g(x) & =-\frac{D_{z} f}{f}, & D_{x} D_{z} g(x) & =-\frac{f}{f^{\prime}} \cdot \frac{f^{\prime \prime} D_{z}(f)-f^{\prime} D_{z}\left(f^{\prime}\right)}{\left(f^{\prime}\right)^{2}}, \\
D_{\tau} g(x) & =-\frac{D_{\tau} f}{f^{\prime}}, & D_{x} D_{\tau} g(x) & =-\frac{f}{f^{\prime}} \cdot \frac{f^{\prime \prime} D_{\tau}(f)-f^{\prime} D_{\tau}\left(f^{\prime}\right)}{\left(f^{\prime}\right)^{2}},
\end{aligned}
$$

where on the right-hand side we have omitted the argument $\tilde{x}$ in $f$ and its derivatives.

After changing variables and clearing denominators we find that the vanishing of (8.17) is equivalent to

$$
B(x, y) C(x, y)+\left(f(x) f^{\prime}(y)-f^{\prime}(x) f(y)\right) \cdot D(x, y)=0,
$$

where we have written $x, y$ for $\tilde{x}, \tilde{y}$ and

$$
\begin{aligned}
B(x, y)= & f^{\prime \prime}(x) f(x) f^{\prime}(y)^{2}-f^{\prime \prime}(y) f(y) f^{\prime}(x)^{2}, \\
C(x, y)= & f^{\prime}(x) f^{\prime}(y)-D_{z} f(x) f^{\prime}(y)-f^{\prime}(x) D_{z} f(y)+ \\
& +f(x) D_{\tau} f(y)+D_{\tau} f(x) f(y), \\
D(x, y)= & \left(f^{\prime \prime}(x) D_{z} f(x)-f^{\prime}(x) D_{z} f^{\prime}(x)\right) f^{\prime}(y)^{2}+
\end{aligned}
$$




$$
\begin{aligned}
& +\left(f^{\prime \prime}(y) D_{z} f(y)-f^{\prime}(y) D_{z} f^{\prime}(y)\right) f^{\prime}(x)^{2}+ \\
& -\left(f^{\prime \prime}(x) f(x)-f^{\prime}(x)^{2}\right) f^{\prime}(y) D_{\tau} f(y)+ \\
& -\left(f^{\prime \prime}(x) D_{\tau} f(x)-f^{\prime}(x) D_{\tau} f^{\prime}(x)\right) f(y) f^{\prime}(y)+ \\
& -\left(f^{\prime \prime}(y) f(y)-f^{\prime}(y)^{2}\right) f^{\prime}(x) D_{\tau} f(x)+ \\
& -\left(f^{\prime \prime}(y) D_{\tau} f(y)-f^{\prime}(y) D_{\tau} f^{\prime}(y)\right) f(x) f^{\prime}(x) .
\end{aligned}
$$

Let $\mathcal{F}(x, y, z, \tau)$ be the left-hand side of (8.18). We need to show that $\mathcal{F}=0$. We will argue as in the proof of Theorem 8.1.2. Since it is a polynomial in derivatives of Jacobi forms the function $\mathcal{F}$ is a quasi-Jacobi form of the three elliptic variables $x, y, z$. It is of weight 6 and index $Q(x, y, z)=\frac{3}{2}(2 x+2 y+z) z$. A quick check using the commutation relations in Remark 5.7.3 shows that in the algebra of such quasi-Jacobi forms we have

$$
\frac{\partial}{\partial G_{2}} \mathcal{F}=\frac{\partial}{\partial A(x)} \mathcal{F}=\frac{\partial}{\partial A(y)} \mathcal{F}=0 .
$$

By a direct check (e.g., using a computer) $\mathcal{F}$ has no poles at $y=0$ and vanishes to order 3 at $y=-z$. Hence the ratio

$$
\frac{\mathcal{F}(x, y)}{f(x)^{3} f(y)^{3}}
$$

is holomorphic in $y$ and of index $Q(x, y, z)=-\frac{3}{2} z^{2}$. Hence, it is also 2-periodic (in $x$ and $y$ ). There, it is constant in $y$. But $\mathcal{F}$ is symmetric in $x$ and $y$, so it is also constant in $x$. By checking that the constant term vanishes we are done.

Remark 8.5.6. By Proposition 8.5.1 for all $m, n>0$ the function $\varphi_{m, n}$ is determined by $\varphi_{m}$ and $\varphi_{n}$. Hence (8.15) yields recursive formulas for $\varphi_{m}$, and hence provides an alternative definition of the set of functions $\varphi_{m}$ starting from the initial condition $\varphi_{1}=\Theta(z)$. For example, the case $(n, 1)$ yields

$$
\varphi_{n+1}=D_{z}\left(\varphi_{1}\right) \varphi_{n}+\frac{1}{n} \varphi_{1} D_{z}\left(\varphi_{n}\right)+\sum_{i=1}^{n-1} \frac{1}{i} \varphi_{i, 1} \varphi_{n-i}
$$

\section{Proof of Theorem 8.1.5}

Write $\widetilde{J}_{k, m}$ for the space of holomorphic quasi-Jacobi forms of weight $k$ and index $m$, which is the subspace of holomorphic functions in $\mathbb{C}\left[A, \wp, \wp^{\prime}, G_{2}, G_{4}, \Theta\right]$, see (5.16). We need to show that for all $n \geq 1$ we have

$$
\varphi_{n,-n}-n \in \widetilde{J}_{0, n} .
$$


The idea of the proof is to consider the two expressions for $\frac{\partial}{\partial A} \varphi_{m, n}$ for positive $m, n$ given by (8.13) and (8.14). These terms are equal for $m>0$, and (with minor modifications) they have natural extensions to $m \leq 0$. We will observe that these extensions are both polynomial in $m$ (when fixing $n$ ) up to the same non-polynomial correction term. Hence they are equal for all $m$.

Concretely, let $n>0$ be fixed and let $R(m, n)$ be the right-hand side of (8.13). Then by Corollary 8.4.2 the sum of $R(m, n)$ and

$$
-m z \delta_{m<0}\left(\frac{n}{m+n} D_{\tau}\left(\varphi_{m}\right) \varphi_{n}+\frac{m}{m+n} \varphi_{m} D_{\tau}\left(\varphi_{n}\right)\right)=-m z \delta_{m<0} \varphi_{m, n}
$$

is polynomial in $m$. We write

$$
\widetilde{R}(m, n)=R(m, n)-m z \varphi_{m, n} \delta_{m<0}
$$

to denote this polynomial function.

We now consider the right-hand side of (8.14) and we want to make sense of it for negative $m$. For all $m \geq 0$, with $m \neq n$ in the second line, define

$$
\begin{aligned}
S(m, n) & :=\frac{m n}{m+n} \varphi_{m+n}+\sum_{j=1}^{m-1} \frac{m}{j} \varphi_{m-j, n} \varphi_{j}+\sum_{j=1}^{n-1} \frac{n}{j} \varphi_{m, n-j} \varphi_{j} \\
S(-m, n) & :=\frac{-m n}{-m+n} \varphi_{-m+n}+\sum_{j=1}^{m-1} \frac{m}{j} \varphi_{-m+j, n} \varphi_{j}+\sum_{j=1}^{n-1} \frac{n}{j} \varphi_{-m, n-j} \varphi_{j} .
\end{aligned}
$$

By a direct application of Lemma 8.5.2 the sum

$$
\widetilde{S}(m, n)=S(m, n)-m z \varphi_{m, n} \delta_{m<0}
$$

is polynomial in $m$.

By Proposition 8.5.5 we have $R(m, n)=S(m, n)$, hence $\widetilde{R}(m, n)=\widetilde{S}(m, n)$ for all $m>0$. By polynomiality in $m$ we get $\widetilde{R}(m, n)=\widetilde{S}(m, n)$ for all $m \neq-n$. Thus

$$
\forall m \neq-n: R(m, n)=S(m, n) .
$$

We specialise (8.19) to $m=-n-1$. Since

$$
\begin{aligned}
S(-n-1, n)=- & (n+1) n \varphi_{1}+(n+1) \varphi_{-n, n} \varphi_{1}+ \\
& +\sum_{j=2}^{n} \frac{n+1}{j} \varphi_{-(n+1)+j, n} \varphi_{j}+\sum_{j=1}^{n-1} \frac{n}{j} \varphi_{-(n+1), n-j} \varphi_{j}
\end{aligned}
$$


and $\varphi_{1}=\Theta$, Equation 8.19 yields

$$
\begin{aligned}
\varphi_{-n, n}-n=\frac{1}{(n+1) \Theta}(R(-n-1, n)- \\
\left.\qquad \sum_{j=2}^{n} \frac{n+1}{j} \varphi_{-(n+1)+j, n} \varphi_{j}-\sum_{j=1}^{n-1} \frac{n}{j} \varphi_{-(n+1), n-j} \varphi_{j}\right) .
\end{aligned}
$$

The term in the bracket on the right lies in $\widetilde{J}_{-1, n+1 / 2}$ by inspection. Moreover, again by inspection it vanishes at $z=0$. Hence it must be divisible by $\Theta(z)$ in the algebra of quasi-Jacobi forms. This gives $\varphi_{-n, n}-n \in \widetilde{J}_{0, n}$.

Remark 8.5.7. The proof yields more information. For $m \neq-n$ we have $\frac{\partial}{\partial A} \varphi_{m, n}=$ $R(m, n)$ by (8.13). Using that $R(m, n)=S(m, n)$ we find the anomaly equation

$$
\frac{\partial}{\partial A} \varphi_{-m, n}=\frac{-m n}{-m+n} \varphi_{-m+n}+\sum_{j=1}^{m-1} \frac{m}{j} \varphi_{-m+j, n} \varphi_{j}+\sum_{j=1}^{n-1} \frac{n}{j} \varphi_{-m, n-j} \varphi_{j}
$$

for all $m \neq-n$.

\subsection{Holomorphic anomaly equations II}

We finally derive the precise quasi-Jacobi properties of the functions $\varphi_{m, n}$ in terms of holomorphic anomaly equations.

Proposition 8.6.1. For all $m, n \in \mathbb{Z}$ we have

(a) $\frac{\partial}{\partial G_{2}} \varphi_{m, n}=2 \varphi_{m} \varphi_{n}$.

(b) $\frac{\partial}{\partial A} \varphi_{m, n}=\frac{m n}{m+n} \varphi_{m+n}+\sum_{i+j=m} \frac{|m|}{j} \varphi_{i, n} \varphi_{j}+\sum_{i+j=n} \frac{|n|}{j} \varphi_{m, i} \varphi_{j}$.

In (b) we have the convention that the first term vanishes if $m+n=0$ and that in a sum with condition $i+j=\ell$ (for $\ell=m$ or $\ell=n$ ) we sum over all positive $i, j$ if $\ell$ is positive, and over all negative $i, j$ if $\ell$ is negative.

Proof. Part (a) follows from the defining differential equation (8.6) by applying $\frac{\partial}{\partial G_{2}}$. In part (b) by Proposition 8.5.5 and Remark 8.5.7 we only need to prove the case $m=-n$. For that we restrict ourself to the region $m<0$ and $n>0$. Applying $\frac{\partial}{\partial A}$ to (8.6) yields

$$
D_{z} \varphi_{m, n}+D_{\tau} \frac{\partial}{\partial A} \varphi_{m, n}=\frac{\partial}{\partial A}\left(m n \varphi_{m} \varphi_{n} F+\left(D_{\tau} \varphi_{m}\right)\left(D_{\tau} \varphi_{n}\right)\right) .
$$


The right-hand side and the first term on the left-hand side are polynomial in $m$ and $n$ (in the considered region). Hence $\frac{\partial}{\partial A} \varphi_{m, n}$ is polynomial in $m, n$ up to a constant in $q .^{2}$ Let

$$
T(m, n)=\frac{m \cdot n}{m+n} \varphi_{m+n}+\sum_{i+j=m} \frac{|m|}{j} \varphi_{i, n} \varphi_{j}+\sum_{i+j=n} \frac{|n|}{j} \varphi_{m, i} \varphi_{j}
$$

for all $m \neq-n$ in the region. Using that $\varphi_{m}$ and $\varphi_{m, n}$ are polynomials in $m$ and $n$, the function $T(m, n)$ uniquely extends to $m=-n$. We already know $T(m, n)=$ $\frac{\partial}{\partial A} \varphi_{m, n}$ for all $m \neq-n$ so by the polynomiality of $\frac{\partial}{\partial A} \varphi_{m, n}$ we get for all $m, n$ in the region

$$
T(m, n)=\frac{\partial}{\partial A} \varphi_{m, n}+c_{m, n}(z)
$$

for some $c_{m, n}(z)$ which does not depend on $q$. Specializing to $m=-n$ we see

$$
\frac{\partial}{\partial A} \varphi_{-n, n}+c_{-n, n}(z)=T(-n, n)=-n^{2} z+\sum_{i+j=-n} \frac{n}{j} \varphi_{i, n} \varphi_{j}+\sum_{i+j=n} \frac{n}{j} \varphi_{-n, i} \varphi_{j} .
$$

But $\frac{\partial}{\partial A} \varphi_{-n, n}$ is homogeneous as a quasi-Jacobi form of weight -1 and index $n$. Hence the constant terms in $q$ on both sides must match up and so as desired

$$
\frac{\partial}{\partial A} \varphi_{-n, n}=\sum_{i+j=-n} \frac{n}{j} \varphi_{i, n} \varphi_{j}+\sum_{i+j=n} \frac{n}{j} \varphi_{-n, i} \varphi_{j} .
$$

Remark 8.6.2. Once we know that $\varphi_{n,-n}$ is quasi-Jacobi and know its $A$-derivative it is not difficult to derive a recursive formula for it (ignoring that we already obtained a formula in the proof of Theorem 8.1.5). Indeed, consider the defining differential equation

$$
D_{\tau} \varphi_{m, n}=m n \varphi_{m} \varphi_{n} F+\left(D_{\tau} \varphi_{m}\right)\left(D_{\tau} \varphi_{n}\right) .
$$

Applying $\frac{\partial}{\partial A}$ twice and using the commutation relations we get

$$
\begin{gathered}
(|m|+|n|) \varphi_{m, n}+2 D_{z} \frac{\partial}{\partial A} \varphi_{m, n}+D_{\tau}\left(\frac{\partial}{\partial A}\right)^{2} \varphi_{m, n}= \\
\left(\frac{\partial}{\partial A}\right)^{2}\left(m n \varphi_{m} \varphi_{n} F+\left(D_{\tau} \varphi_{m}\right)\left(D_{\tau} \varphi_{n}\right)\right) .
\end{gathered}
$$

Since $\left(\frac{\partial}{\partial A}\right)^{i} \varphi_{m, n}$ is determined recursively from functions indexed by $m^{\prime}, n^{\prime}$ with $m^{\prime}+n^{\prime}<m+n$, this yields one more formula for $\varphi_{m, n}$.

\footnotetext{
${ }^{2}$ There is a small subtlety here since at first it only follows that $\frac{\partial}{\partial A} \varphi_{m, n}$ is a power series in $z, q$ whose coefficients are polynomial in $m, n$. But then $\frac{\partial}{\partial A} \varphi_{m, n}$ is a quasi-Jacobi form for every $m, n$ so that it actually has to be a power series in $z$ with coefficients which are polynomials with coefficients quasi-modular forms (of determined weight).
} 


\subsection{The classical Kaneko-Zagier equation}

The differential equation introduced by Kaneko and Zagier [KZ98] can be characterised among quadratic differential equations as those for which the solution space is invariant under the modular transformation for the full modular group, so that it is essentially unique [KK03]. If one however considers congruence subgroups, further differential equations of the same type have been found by Kaneko and Koike [KK04]. In this section we give a general construction which takes as input a meromorphic Jacobi form of weight -1 and gives as output a differential equation of Kaneko-Zagier type. The two Kaneko-Zagier equations above and our case studied in this chapter are all given by this construction. (A certain differential equation for index 1 Jacobi forms was studied by Kiyuna [Kiy16] and was called a Kaneko-Zagier type equation. However, since it is of fourth order it does not fit our framework.)

\section{A general construction}

A general recipe to construct Kaneko-Zagier type differential equations is as follows. Let $g$ be a meromorphic Jacobi form of weight -1 (e.g., $g$ is a meromorphic modular form of weight -1 ). Define

$$
E=\frac{D_{\tau} g}{g} \quad \text { and } \quad H=\frac{D_{\tau}^{2} g}{g} .
$$

By construction $E$ and $H$ are meromorphic quasi-Jacobi forms. Observe that as $g$ is of weight -1 , it follows that $\frac{\partial}{\partial G_{2}} H=0$. It is not necessarily true that $\frac{\partial}{\partial A} H$ vanishes.

For all $m \geq 1$ we consider the differential equation

$$
D_{\tau}^{2} g_{m}=m^{2} H(\tau) g_{m} .
$$

To obtain the connection to the classical presentation, we set $m=k+1$, and consider

$$
f_{k}=g_{k+1} / g^{k+1},
$$

which is of weight $k$. The corresponding differential equation for $f_{k}$ reads

$$
D_{\tau}^{2} f_{k}+2(k+1) E D_{\tau} f_{k}+k(k+1)\left(E^{2}-H\right) f_{k}=0 .
$$

For this choice of $g$ (and hence of $E$ ), we define a modified Serre derivative $\theta_{g}$ on quasi-Jacobi forms by

$$
\theta_{g}=D_{\tau}+E W
$$

where the operator $W$ multiplies a quasi-Jacobi form by its weight. The operator $\theta_{g}$ is a derivation vanishing on $g$. Moreover, the above differential equation can be rewritten as

$$
\theta_{g}^{2} f_{k}=H W(W+2) f_{k}
$$


We give several examples:

(0) In this chapter we considered the case $g(z)=\Theta(z)$ (which contains the cases $g=\Theta(a)$ for any $a \in \mathbb{Q})$

(1) For the classical Kaneko-Zagier equation we let

$$
g(\tau)=\frac{1}{\eta(\tau)^{2}}
$$

and get $H(\tau)=E_{4}(\tau) / 144$. The operator $\theta_{g}$ is the Serre derivative.

(2) For the differential equation studied in [KK04] we take

$$
g(\tau)=\frac{1}{\eta(\tau) \eta(2 \tau)}
$$

and get

$$
E(\tau)=\frac{1}{24}\left(E_{2}(\tau)+2 E_{2}(2 \tau)\right), \quad 2^{6} H(\tau)=\frac{1}{5}\left(E_{4}(\tau)+4 E_{4}(2 \tau)\right) .
$$

The operator $\theta_{g}$ matches the derivative operator of [KK04, Sec. 2]. Unpublished work by Tomoaki Nakaya [Nak19, Section 3.5] shows that in this case

$$
f_{k}=\operatorname{Res}_{z=0}\left(D_{z} \frac{\Theta(2 \tau, 2 z)}{\Theta(\tau, z)^{2}}\right)^{-(k+1) / 2}
$$

is a solution of the differential equation (8.21) for all $k \geq 1$.

Provided with these examples (and more in [Nak19]), a natural question we do not answer in this thesis is the following.

Question 8.7.1. Does there exists a (natural) lift $\psi_{g}$ of meromorphic modular forms $g$ of weight -1 such that

(i) $g_{m}=\operatorname{Res}_{x=0} \psi_{g}(x)^{m}$ solves the differential equation (8.20) given by $g$;

(ii) for some $n \in \mathbb{Z}$ the function $\psi_{g}^{n}$ is a meromorphic Jacobi form?

\section{Recursive construction of the solutions}

Let $f_{k}$ and $f_{l}$ be two solutions of (8.21) of weight $k$ and $l$ respectively. We write

$$
[f, h]:=k \theta_{g}(f) h-l f \theta_{g}(h)=k D_{\tau}(f) h-l f D_{\tau}(h),
$$

which specialises to the first Rankin-Cohen bracket on modular forms. 
Proposition 8.7.2. We have

$$
\begin{aligned}
& \theta_{g}\left[f_{k}, f_{l}\right]=\frac{k-l}{l+2}\left[f_{k}, \theta_{g}\left(f_{l}\right)\right], \\
& \theta_{g}^{2}\left[f_{k}, f_{l}\right]=(k-l)(k-l-2) H\left[f_{k}, f_{l}\right]+k(k-l) f_{k}\left[f_{l}, H\right] .
\end{aligned}
$$

Proof. This follows from a direct computation.

Corollary 8.7.3. Suppose that $\left[f_{l}, H\right]=0$. Then

$$
\left[f_{k}, f_{l}\right] g^{2 l+4} \text { and }\left[f_{k} g^{2 k+2}, f_{l}\right] g^{-2 k-2}
$$

are solutions of (8.21) of weight $k-l-2$ and $k+l+2$ respectively.

Hence if a function $f_{l}$ as in the corollary exists, then from any given solution we can recursively write down solutions of (8.21) with weight in the same residue class modulo $l$.

Example 8.7.4. For the classical Kaneko-Zagier equation we can take $f_{l}=E_{4}$. Then indeed $\left[f_{l}, H\right]=0$, so that if $f_{k}$ is a solution we have that $\left[f_{k}, E_{4}\right] / \Delta$ is a solution of weight $k-6$, and $\left[f_{k} \eta^{-4 k-4}, E_{4}\right] \eta^{4 k+4}$ is a solution of weight $k+6$. The first of these equations can also be found in [KK03, Proposition 1(i)].

Example 8.7.5. For the Kaneko-Zagier equation in Example (2) we can take $f_{l}=$ $2 E_{2}(2 \tau)-E_{2}(\tau)$. Then, indeed $\left[f_{l}, H\right]=0$, so solutions can be constructed 4periodically, compare also with [KK04].

Remark 8.7.6. For the differential equation of Example (0), considered in this chapter, it turns out that the recursive structure described in Corollary 8.7.3 does not exist. To see this, suppose (for our general family of Kaneko-Zagier equations) that there exists a solution $f_{l}$ and that moreover we have $\left[f_{l}, H\right]=0$. Then the condition $\left[f_{l}, H\right]=0$ is equivalent to

$$
\theta_{g} f_{l}=\frac{l}{4}\left(\frac{D_{\tau}^{3} g}{D_{\tau}^{2} g}+3 \frac{D_{\tau} g}{g}\right) f_{l} .
$$

Applying $\theta_{g}$ to this equation and using the differential equation for the left-hand side, we obtain

$$
\begin{aligned}
16(l+2) \frac{D_{\tau}^{2} g}{g}=4 \frac{D_{\tau}^{4} g}{D_{\tau}^{2} g}+12 & \frac{D_{\tau}^{2} g}{g}+(l-4)\left(\frac{D_{\tau}^{3} g}{D_{\tau}^{2} g}\right)^{2}+ \\
& +2(3 l+4) \frac{D_{\tau}^{3} g}{D_{\tau}^{2} g} \frac{D_{\tau} g}{g}+3(3 l+4)\left(\frac{D_{\tau} g}{g}\right)^{2} .
\end{aligned}
$$


For $g=\eta^{-2}$ this equation is only satisfied if $l=4$, and for $g=(\eta(\tau) \eta(2 \tau))^{-1}$ only if $l=2$. However, for $g=\Theta(z)$ this equation is never satisfied, so Corollary 8.7.3 cannot be applied. This points to a structural difference between the Kaneko-Zagier equation in this chapter and the already existing Kaneko-Zagier equations, which, like Question 8.7.1, is a possibly interesting topic. 
Appendix $A$

\section{Tables of functions on partitions and quasimodular forms}

\section{A.1 Shifted symmetric harmonic polynomials up to weight 10}

We list all harmonic polynomials $h_{\lambda}$ of even weight at most 10 . The corresponding $q$ brackets $\left\langle h_{\lambda}\right\rangle_{q}$ are computed by the algorithm prescriped by Zagier [Zag16] using SageMath [Sage17].

\begin{tabular}{|c|c|c|}
\hline$\lambda$ & $h_{\lambda}$ & $\left\langle h_{\lambda}\right\rangle_{q}$ \\
\hline () & 1 & 1 \\
\hline$(4)$ & $\frac{27}{4}\left(Q_{2}^{2}+2 Q_{4}\right)$ & $\frac{9}{320} Q$ \\
\hline$(6)$ & $\frac{225}{4}\left(63 Q_{6}+9 Q_{2} Q_{4}+Q_{2}^{3}\right)$ & $-\frac{55}{384} R$ \\
\hline$(3,3)$ & $\frac{225}{4}\left(63 Q_{3}^{2}-108 Q_{2} Q_{4}+2 Q_{2}^{3}\right)$ & $\frac{115}{384} R$ \\
\hline$(8)$ & $\frac{19845}{16}\left(3960 Q_{8}+360 Q_{2} Q_{6}+20 Q_{2}^{2} Q_{4}+Q_{2}^{4}\right)$ & $\frac{19173}{4096} Q^{2}$ \\
\hline$(5,3)$ & $\frac{19845}{2}\left(495 Q_{3} Q_{5}+45 Q_{2} Q_{3}^{2}-1350 Q_{2} Q_{6}-50 Q_{2}^{2} Q_{4}+2 Q_{2}^{4}\right)$ & $-\frac{2415}{128} Q^{2}$ \\
\hline$(4,4)$ & $\frac{297675}{8}\left(132 Q_{4}^{2}+24 Q_{2} Q_{3}^{2}-440 Q_{2} Q_{6}-28 Q_{2}^{2} Q_{4}+Q_{2}^{4}\right)$ & $-\frac{38241}{2048} Q^{2}$ \\
\hline$(10)$ & $\frac{382725}{8}\left(450450 Q_{10}+30030 Q_{2} Q_{8}+1155 Q_{2}^{2} Q_{6}+35 Q_{2}^{3} Q_{4}+Q_{2}^{5}\right)$ & $-\frac{2053485}{4096} Q R$ \\
\hline \multirow[t]{2}{*}{$(7,3)$} & $\frac{1913625}{8}\left(90090 Q_{3} Q_{7}+6006 Q_{2} Q_{3} Q_{5}-336336 Q_{2} Q_{8}+\right.$ & \\
\hline & $\left.+231 Q_{2} Q_{3}^{2}-12936 Q_{2}^{2} Q_{6}-112 Q_{2}^{3} Q_{4}+10 Q_{2}^{5}\right)$ & $\frac{11975985}{4096} Q R$ \\
\hline \multirow[t]{2}{*}{$(6,4)$} & $\frac{13395375}{8}\left(12870 Q_{4} Q_{6}+1716 Q_{2} Q_{3} Q_{5}+858 Q_{2} Q_{4}^{2}-96096 Q_{2} Q_{8}+\right.$ & \\
\hline & $\left.+132 Q_{2}^{2} Q_{3}^{2}-6501 Q_{2}^{2} Q_{6}-89 Q_{2}^{3} Q_{4}+5 Q_{2}^{4}\right)$ & $\frac{21255885}{4096} Q R$ \\
\hline \multirow[t]{2}{*}{$(5,5)$} & $\frac{8037225}{4}\left(10725 Q_{5}^{2}+1430 Q_{2} Q_{3} Q_{5}+1430 Q_{2} Q_{4}^{2}-10010 Q_{2} Q_{8}+\right.$ & \\
\hline & $\left.+165 Q_{2}^{2} Q_{3}^{2}-7700 Q_{2}^{2} Q_{6}-120 Q_{2}^{3} Q_{4}+6 Q_{2}^{5}\right)$ & $\frac{7759395}{1024} Q R$ \\
\hline \multirow[t]{2}{*}{$(4,3,3)$} & $\frac{13395375}{8}\left(12870 Q_{3}^{2} Q_{4}-34320 Q_{2} Q_{3} Q_{5}+10296 Q_{2} Q_{4}^{2}+\right.$ & \\
\hline & $\left.+363 Q_{2}^{2} Q_{3}^{2}++55440 Q_{2}^{2} Q_{6}-376 Q_{2}^{3} Q_{4}+10 Q_{2}^{5}\right)$ & $-\frac{16583805}{4096} Q R$ \\
\hline
\end{tabular}


In case $|\lambda|$ is odd the harmonic polynomials $h_{\lambda}$ up to weight 9 are given in the following table. The $q$-bracket of odd degree (harmonic) polynomials is zero, hence trivially modular.

\begin{tabular}{ll}
$\lambda$ & $h_{\lambda}$ \\
\hline \hline$(3)$ & $-\frac{9}{4} Q_{3}$ \\
$(5)$ & $-\frac{135}{4}\left(5 Q_{5}+Q_{2} Q_{3}\right)$ \\
$(7)$ & $-\frac{14175}{16}\left(126 Q_{7}+14 Q_{2} Q_{5}+Q_{2}^{2} Q_{3}\right)$ \\
$(4,3)$ & $-\frac{99225}{16}\left(18 Q_{3} Q_{4}-40 Q_{2} Q_{5}+Q_{2}^{2} Q_{3}\right)$ \\
$(9)$ & $-\frac{297675}{8}\left(7722 Q_{9}+594 Q_{2} Q_{7}+27 Q_{2}^{2} Q_{5}+Q_{2}^{3} Q_{3}\right)$ \\
$(6,3)$ & $-\frac{893025}{4}\left(1287 Q_{3} Q_{6}+99 Q_{2} Q_{3} Q_{4}-4158 Q_{2} Q_{7}-162 Q_{2}^{2} Q_{5}+5 Q_{2}^{3} Q_{3}\right)$ \\
$(5,4)$ & $-\frac{8037225}{8}\left(286 Q_{4} Q_{5}+66 Q_{2} Q_{3} Q_{4}-1540 Q_{2} Q_{7}-117 Q_{2}^{2} Q_{5}+3 Q_{2}^{3} Q_{3}\right)$ \\
$(3,3,3)$ & $-\frac{893025}{4}\left(1287 Q_{3}^{3}-3564 Q_{2} Q_{3} Q_{4}+3240 Q_{2}^{2} Q_{5}+10 Q_{2}^{3} Q_{3}\right)$ \\
\hline
\end{tabular}

\section{A.2 Examples of quasimodular generating series of Hurwitz numbers}

Next, we provide some examples of the quasimodular forms $\sum_{d} h_{k, l, m}^{g, d}(\mu) q^{d}$, computed using SageMath [Sage17]. See [RY10, Section 10] for an extensive list of quasimodular forms corresponding to simple Hurwitz numbers $(l=m=0, \mu=())$.

\section{Genus $g=2$}

$$
\begin{aligned}
\sum_{d} h_{2,0,0}^{2, d}() q^{d} & =\frac{1}{2^{6} 3^{4} 5}\left(5 P^{3}-3 P Q-2 R\right) \\
& =2 q^{2}+16 q^{3}+60 q^{4}+160 q^{5}+360 q^{6}+672 q^{7}+1240 q^{8}+1920 q^{9}+O\left(q^{10}\right) \\
\sum_{d} h_{0,2,0}^{2, d}() q^{d} & =\frac{1}{2^{7} 3^{4} 5}\left(5 P^{3}-3 P Q-2 R+45 P^{2}+18 Q+90 P-153\right) \\
& =2 q^{2}+13 q^{3}+44 q^{4}+109 q^{5}+235 q^{6}+422 q^{7}+760 q^{8}+1151 q^{9}+O\left(q^{10}\right) \\
\sum_{d} h_{0,0,2}^{2, d}() q^{d} & =\frac{1}{2^{7} 3^{4} 5}\left(5 P^{3}-3 P Q-2 R-45 P^{2}-18 Q-90 P+153\right) \\
& =3 q^{3}+16 q^{4}+51 q^{5}+125 q^{6}+250 q^{7}+480 q^{8}+769 q^{9}+O\left(q^{10}\right)
\end{aligned}
$$


Genus $g=3$

$$
\begin{aligned}
& \sum_{d} h_{2,0,0}^{3, d}(3) q^{d} \\
& =\frac{1}{2^{13} 3^{5} 5^{2}}\left(-875 P^{5}+1775 P^{3} Q-10 P^{2} R-894 P Q^{2}+4 Q R+\right. \\
& \left.\quad 750 P^{4}-1710 P^{2} Q+60 P R+900 Q^{2}+135 P^{3}-81 P Q-54 R\right) \\
& =36 q^{3}+540 q^{4}+3606 q^{5}+15726 q^{6}+53298 q^{7}+149142 q^{8}+367920 q^{9}+O\left(q^{10}\right) \\
& \sum_{d} h_{0,2,0}^{3, d}(3) q^{d} \\
& =\frac{1}{2^{14} 3^{5} 5^{2}}\left(-875 P^{5}+1775 P^{3} Q-10 P^{2} R-894 P Q^{2}+4 Q R+\right. \\
& \quad-2625 P^{4}-630 P^{2} Q+\frac{23460}{7} P R+\frac{648}{7} Q^{2} \\
& \left.\quad+2835 P^{3}+1359 P Q-5814 R+3150 P^{2}-4608 Q+7020 P-4131\right) \\
& =27 q^{3}+369 q^{4}+2337 q^{5}+9795 q^{6}+32307 q^{7}+88446 q^{8}+214536 q^{9}+O\left(q^{10}\right) \\
& \sum_{d} h_{0,0,2}^{3, d}(3) q^{d} \\
& =\frac{1}{2^{14} 3^{5} 5^{2}}\left(-875 P^{5}+1775 P^{3} Q-10 P^{2} R-894 P Q^{2}+4 Q R+\right. \\
& \quad+4125 P^{4}-2790 P^{2} Q+\frac{22620}{7} P R+\frac{11952}{7} Q^{2}+ \\
& \left.\quad-2565 P^{3}-1521 P Q+5706 R-3150 P^{2}+4608 Q-7020 P+4131\right) \\
& =9 q^{3}+171 q^{4}+1269 q^{5}+5931 q^{6}+20991 q^{7}+60696 q^{8}+153384 q^{9}+O\left(q^{10}\right)
\end{aligned}
$$

\section{A.3 Double moment functions up to weight 4}

For all basis elements $f \in \mathcal{T}_{\leq 4}$ in the basis provided by Theorem 4.8.9, we compute its representation in the basis consisting of double moment function and the quantities $\langle f\rangle_{\boldsymbol{u}},\langle f\rangle_{q}, D(f), \mathfrak{d}(f)$ and $\mathcal{M}(f)$.

\section{Weight at most 2}

\begin{tabular}{l||l|l|l}
$f$ & 1 & $T_{1,1}$ & $T_{0,2}$ \\
\hline$\langle f\rangle_{\boldsymbol{u}}$ & 1 & $-\frac{1}{24}+\sum_{m, r \geq 1} m u_{m}^{r}$ & $-\frac{1}{24}+\sum_{m, r \geq 1} r u_{m}^{r}$ \\
$\langle f\rangle_{q}$ & 1 & $G_{2}$ & $G_{2}$ \\
$D(f)$ & 0 & $T_{2,2}$ & $T_{1,3}$ \\
$\mathfrak{d}(f)$ & 0 & $-\frac{1}{2}$ & $-\frac{1}{2}$ \\
$\mathcal{M}(f)$ & 1 & $X_{1,1}-\frac{1}{24}$ & $X_{0,2}+X_{0,1}-\frac{1}{24}$
\end{tabular}




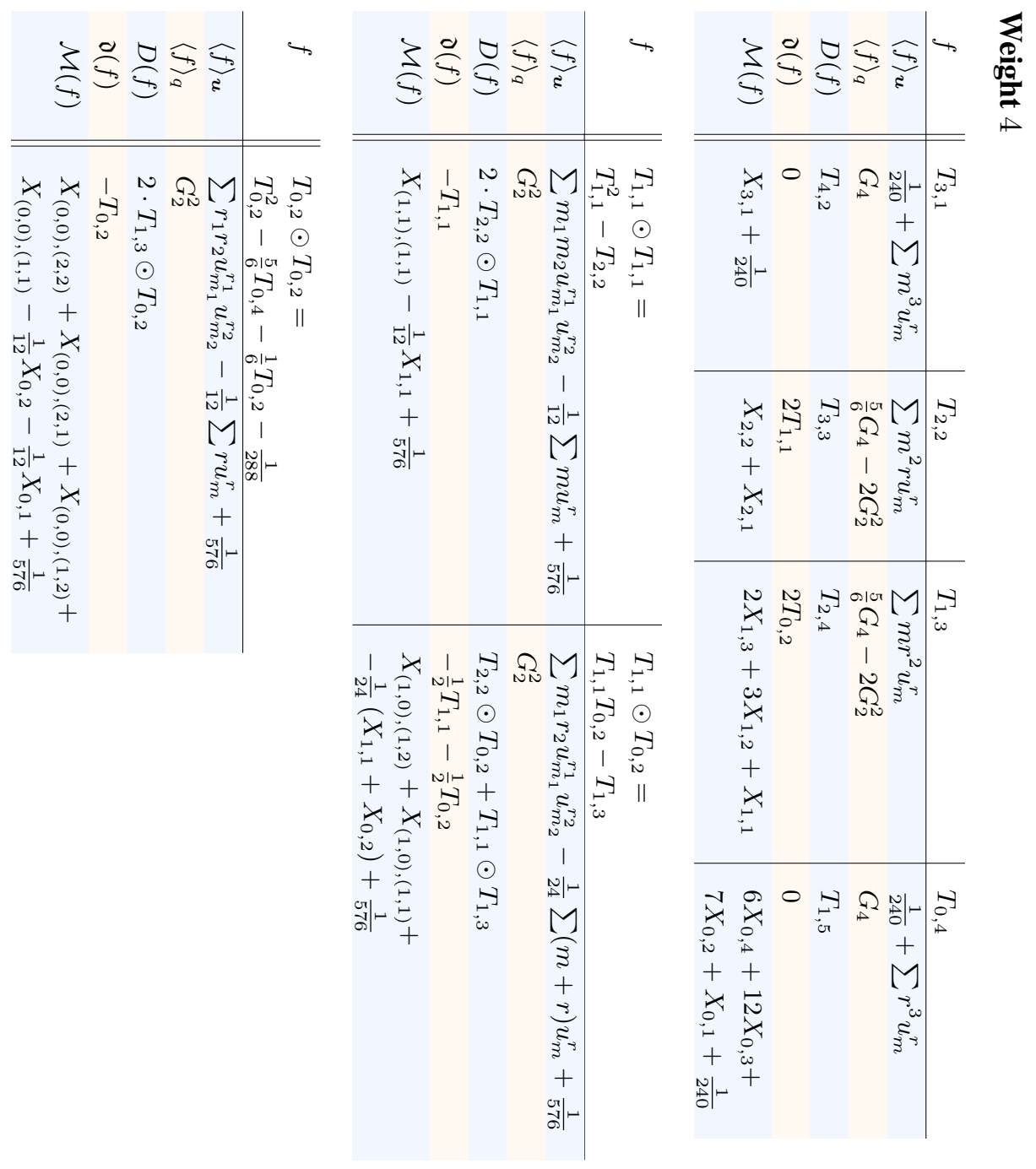




\section{Samenvatting}

Het is lunchtijd! Terwijl jij jouw broodtrommeltje tevoorschijn haalt, komt JanWillem aangelopen met een zak brood en een pot vol smeersel. Wat is dat voor smeersel? Is het chocopasta? Of heeft hij vandaag weer pindakaas? 'Je mag wel een beetje proeven, hoor', zegt hij wanneer hij je naar de pot ziet staren. 'Het zijn de laatste resultaten van mijn proefschrift.' Je neemt een hapje. Het smaakt een beetje als gekristalliseerde honing en ook als spekkoek; hij noemt het een quasimodulaire vorm. 'Zelfgemaakt,' zegt hij trots. Nog voordat je hem een verdere vraag kan stellen, begint hij al te vertellen: 'Je neemt een bepaalde hoeveelheid partities, een soort tetris-chocoladerepen. Even kloppen met een speciaal soort garde-de $q$-haak-en... tadaah: een quasimodulaire vorm! Vandaag is het $E_{2}$, want ik had van elke partitie evenveel gram als zijn eigen grootte genomen, maar ik had ook best een bepaalde macht van alle delen kunnen nemen.' 'Eh, ik volg het niet helemaal', onderbreekt iemand anders hem. 'Wat zijn dat voor ingrediënten, partities? En wat is nu precies het recept?' 'Lekker is het in ieder geval wel,' denk je. Hier wil je meer van (w)eten!

In dit proefschrift besturen we variaties op de Bloch-Okounkov stelling: een soort recept om quasimodulaire vormen te maken uit partities. Een recept begint altijd met een aantal ingrediënten, geeft vervolgens een bereidingswijze om tot een resultaat te komen en sluit af met enkele variatietips. Evenzo begint deze samenvatting met het introduceren van partities, vervolgens definiëren we de $q$-haak en de quasimodulaire vormen die we met dit recept bereiden. We sluiten af met enkele variatietips, dat wil zeggen: de eigenlijke resultaten van dit proefschrift. 


\section{Ingrediënten: partities}

Een partitie is een manier om een geheel getal te schrijven als de som van positieve gehele getallen. Zo is bijvoorbeeld $3+2$ een partitie van 5 . Alle partities van het getal 5 zijn als volgt:

$$
5,4+1,3+2,3+1+1,2+2+1,2+1+1+1, \quad 1+1+1+1+1 .
$$

Andere sommen zijn niet mogelijk, behalve dat we ook $2+3$ zouden kunnen schrijven in plaats van $3+2$. We spreken af dat we dat we in zo'n geval $3+2$ en $2+3$ als dezelfde partitie beschouwen, omdat de volgorde van de getallen in een som niet uitmaakt. De 'tetris-chocoladereep', beter gezegd het Young diagram, die hoort bij deze partitie is

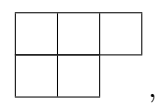

want de eerste rij in dit diagram telt 3 hokjes en de tweede rij telt 2 hokjes. In het algemeen construeer je het Young diagram van een partitie door de getallen in de bijbehorende som door een rij van evenveel hokjes te vervangen; in het voorbeeld van de partities van 5 hierboven krijg je dan:
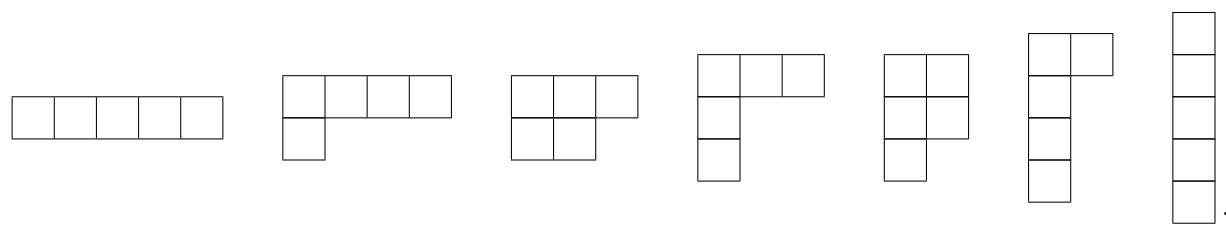

Merk op dat het aantal hokjes in alle bovenstaande diagrammen gelijk is aan 5, dit getal noemen we de grootte van een partitie.

De ingrediënten van ons recept zijn dus partities: niet alleen van 5 , maar partities van alle mogelijke gehele getallen. We moeten ook nog vertellen wat het gewicht is van elke partitie voor het recept: het maakt namelijk heel veel uit of je een brood bakt van $200 \mathrm{~g}$ bloem en $4 \mathrm{~g}$ zout of $4 \mathrm{~g}$ bloem en $200 \mathrm{~g}$ zout. Dat is precies waar dit proefschrift over gaat: welke combinatie van gewichten zorgt er voor dat het recept een (heerlijke) quasimodulaire vorm als uitkomst heeft? We geven twee voorbeelden van 'goede' gewichten.

Het recept van het smeersel in het begin nam 5 gram van elke bovenstaande partitie van het getal 5 . Dat was echter niet alles, ook van de partities van 6 (probeer die eens zelf te tekenen - het zijn er 11) werd van elk 6 gram genomen, etc. In andere woorden: van elke partitie van een geheel getal $n$ werd precies $n$ gram genomen. Oftewel, het gewicht van elke partitie is gelijk aan de grootte van die partitie, in wiskundige notatie: $g(\lambda)=\lambda_{1}+\lambda_{2}+\ldots \lambda_{r}$, waar $g$ het gewicht is van een partitie $\lambda=\lambda_{1}+\lambda_{2}+\ldots+\lambda_{r}$ bestaande uit $r$ delen. 
Een tweede, iets ingewikkelder recept is als volgt. Neem als gewichtsfunctie

$$
g(\lambda)=\sum_{i}\left(\left(\lambda_{i}-i+\frac{1}{2}\right)^{2}-\left(-i+\frac{1}{2}\right)^{2}\right) .
$$

Hoeveel gewicht nemen we dan van de partitie $\lambda=3+2$ ? Nou,

$$
g(3+2)=\left(3-1+\frac{1}{2}\right)^{2}-\left(-1+\frac{1}{2}\right)^{2}+\left(2-1+\frac{1}{2}\right)^{2}-\left(-2+\frac{1}{2}\right)^{2}=6
$$

gram. Het is precies deze gewichtsfunctie, alsmede combinaties waar het kwadraat is vervangen door een andere macht, die gebruikt worden in het recept van de BlochOkounkov stelling.

Opmerking. Bij het tellen van zogenaamde overdekkingen van twee-dimensionale meetkundige objecten (oppervlakken) stuiten wiskundigen als vanzelf op bepaalde hoeveelheden partities. Dit soort tellingen begon met het werk van de wiskundige Hurwitz aan het einde van de 19e eeuw en vormt een groot onderzoeksgebied in de huidige wiskunde en natuurkunde. Ook dit proefschrift bevat enkele resulten over zulke vragen, maar daar zal ik in deze korte samenvatting niet op ingaan. Mocht het dus moeilijk zijn om aan de ingrediënten te komen, vraag dan een aftellende meetkundige of hij nog wat partities heeft!

\section{Bereidingswijze: de $q$-haak}

Neem dus $g(\lambda)$ gram van elke partitie $\lambda$, waarbij $g$ een gewichtsfunctie is. Net als bij de bereiding van spekkoek maken we de quasimodulaire vorm laag voor laag. In principe wordt elke laag gevormd door partities van een vaste grootte: de vijfde laag wordt gemaakt door alle partities van grootte 5 samen te nemen. Echter, helemaal gescheiden zijn deze lagen niet: ook de eerste, tweede, derde en vierde laag laten hun sporen na op de vijfde laag. Wiskundigen noemen het resultaat van dit proces de $q$-haak van $g$ en schrijven dit als

$$
\frac{\sum_{n=1}^{\infty} \sum_{\lambda \vdash n} g(\lambda) q^{n}}{\sum_{n=1}^{\infty} \sum_{\lambda \vdash n} q^{n}} .
$$

Hier betekent $\sum_{\lambda \vdash n} g(\lambda)$ dat we alle partities van het getal $n$ samen nemen, en $q^{n}$ geeft aan dat we bezig zijn met de $n$-de laag (dat wil zeggen, de $n$-de Fourier coëffciënt). De noemer van deze uitdrukking zorgt voor het effect van het sporen nalaten van lagere lagen in de hogere. Deze noemer $\sum_{n=1}^{\infty} \sum_{\lambda \vdash n} q^{n}$ is de telfunctie van partities:

$$
\sum_{n=1}^{\infty} \sum_{\lambda \vdash n} q^{n}=1+q+2 q^{2}+3 q^{3}+5 q^{4}+7 q^{5}+\ldots
$$


Het getal 7 staat hierin voor de 7 partities van 5 , hierboven opgesomd, en hetzelfde geldt voor voor de andere coefficiënten. De wiskundige Euler heeft in de $18 \mathrm{e}$ eeuw al een gesloten formule gegeven voor deze reeks. Je zou dus kunnen zeggen dat de resultaten in dit proefschrift die gaan over de breuk (A.1) een verfijning gegeven van een welbekende formule van Euler.

Opmerking. De breuk (A.1) kunnen we ook interpreteren als de door de natuurkundigen Maxwell and Boltzmann in de 19e eeuw geïntroduceerde toestandsom van een meetbare grootheid $g$ in een systeem. In dat geval is $\lambda$ een van de vele microstaten, het getal $n$ de bijbehorende energie en het getal $q$ een functie van de temperatuur. Deze toestandsom geeft alle macroscopische informatie over het systeem, bijvoorbeeld het volume en de druk.

\section{Resultaat: een quasimodulaire vorm}
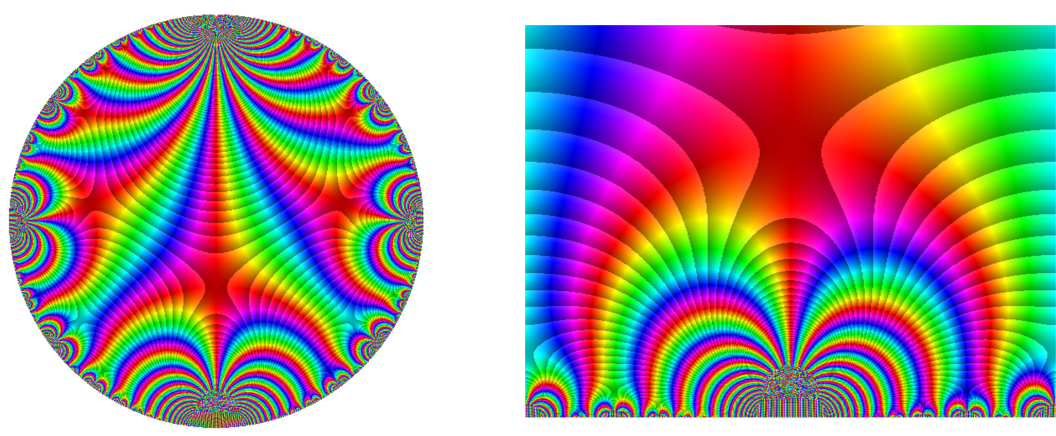

De quasimodulaire vorm die volgens bovenstaand recept is verkregen kan men zoals hierboven visualiseren ${ }^{1}$. Het is duidelijk dat quasimodulaire vormen een opmerkelijk symmetrie bezitten! Deze doet denken aan de kristalstructuur van honing, de regelmatige structuur van een bijenraat, en ook het werk Hemel en hel van M. C. Escher.

Daarnaast voelen quasimodulaire vormen glad aan, zonder enige randjes of oneffenheden (dat wil zeggen, ze zijn holomorf). Deze 'gladheid' en symmetrie zijn de belangrijkste zichtbare eigenschappen van quasimodulaire vormen als functie, waaruit nog twee andere belangrijke eigenschappen te herleiden zijn:

(i) Quasimodulaire vormen kan je bij elkaar optellen, aftrekken en met elkaar vermenigvuldigen. Het resultaat is dan een nieuwe quasimodulaire vorm.

\footnotetext{
${ }^{1}$ zie [LD20] voor hoe men deze afbeeldingen maakt en wat ze precies weergeven.
} 
Bovendien kan je quasimodulaire vormen differentiëren, ook dan is het resultaat een quasimodulaire vorm.

(ii) Er zijn betrekkelijk weinig quasimodulaire vormen. Alle quasimodulaire vormen zijn namelijk een combinatie van drie 'bouwstenen', die we $G_{2}, G_{4}$ en $G_{6}$ noemen. Het grote voordeel hiervan is dat we quasimodulaire vormen heel makkelijk kunnen herkennen en dat we hun eigenschappen heel goed begrijpen. Het nadeel hiervan is dat als je een kleine fout maakt in de gewichten van partities in het bereidingsproces het resultaat geen quasimodulaire vorm meer is.

\section{Variatietips, ofwel de resultaten in dit proefschrift}

De belangrijkste resultaten in dit proefschrift zijn de antwoorden op de vragen in Sectie 1.6. We zouden deze vragen als volgt kunnen parafraseren:

(1) Zoals gezegd kan je quasimodulaire vormen met elkaar vermenigvuldigen, maar het is zelfs zo dat je de recepten van Bloch en Okounkov met elkaar kan vermenigvuldigen (door de gewichten van elke partitie in de recepten te vermenigvuldigen). Bestaan er, naast de recepten van Bloch en Okounkov, nog meer recepten van quasimodulaire vormen met deze eigenschap?

(2) Er zijn verschillende 'niveaus' van symmetrie voor quasimodulaire vormen. Tot nu toe hebben we ons beperkt tot het geval met de meeste symmetrie. Kunnen we ook recepten vinden waarbij de uitkomst iets minder symmetrie heeft?

(3) Bestaan er ook recepten waarbij de uitkomst meer symmetrie oplevert? Immers, sommige quasimodulaire vormen zijn in feite modulaire vormen (de vormen die een combinatie zijn van $G_{4}$ en $G_{6}$, zonder $G_{2}$ ). Bestaat er een manier om te voorspellen welke recepten modulaire vormen opleveren?

In dit proefschrift leggen we uit hoe en waarom het antwoord op deze drie vragen 'ja' is. 



\section{Dankwoord}

Ik ben zeer gezegend met twee excellente wiskundigen als mijn promotoren, alsmede met hun begeleiding in het gehele proces van het schrijven van dit proefschrift. Gunther, jij hebt een uitstekende visie op wat er voor nodig is om een masterstudent een zelfstandig onderzoeker te laten worden. Ik waardeer onze wekelijkse (!) gesprekken en jouw inzet voor een gezonde sfeer en uitwisseling op het mathematisch instituut. Ik bewonder jouw vermogen om, onafhankelijk van de hoeveelheid kennis die je hebt van het onderzoeksgebied, de juiste vragen te stellen. Dit heeft een grote invloed gehad op mijn onderzoek, vanaf het moment dat ik mijn eerste berekingen uitvoerde aan de Mahler maat tot en met resultaten die pas na dit proefschrift zullen verschijnen. Don, ik geniet ervan om van jouw artikelen te leren, te luisteren naar jouw voordrachten, maar bovenal van de vele inzichten die voorvloeien uit onze gesprekken. De wiskundige inhoud van al deze gesprekken heb ik steeds samengevat en bewaard, opdat ik er later vele vruchten van kan plukken. Jouw opbouwende kritiek op mijn schrijfstijl heeft niet alleen geleid tot vele grote verbeteringen in mijn artikelen en in dit proefschrift, maar ook tot een beter zicht op hoe mijn resultaten passen in een groter kader van de wiskunde. Dank aan jullie beiden voor het plezierige vooruitzicht om vanaf nu verder te onderzoeken gebruikmakend van alles wat ik van jullie heb geleerd.

Frits Beukers, Masanobu Kaneko and Hiroyuki Ochiai, thank you for interesting comments and for pointing out minor mistakes in a previous version of this thesis. Many thanks also to the rest of the thesis committee, Kathrin Bringmann, Martin Möller and Sameer Murthy, for reading this thesis carefully.

Ik heb genoten van de vrijheid en verantwoordelijk om te promoveren vanuit een zelfgeschreven onderzoeksvoorstel. Dank aan het NWO die hier geld voor heeft vrijgemaakt; ik hoop dat dergelijke promotieplekken ook in de toekomst beschikbaar blijven!

Sergej, grazie per le tue risposte a tutte le mie domande sulla geometria enumerativa, grazie per il tuo ruolo di paranimf e grazie per la nostra amicizia. Harry, dank voor onze (niet-)wiskundige gesprekken en het goede voorbeeld dat je me geeft in 
het lesgeven. Joost en Nick, dank voor de vele tijd samen in ons kantoor. Roelof Bruggeman, Sergej, Berend and Mar, thank you for our conversations on modular forms and more. Thanks to the rest of the number theory group, Carlo, Damaris, Jens, Lasse, Lola, Marieke, Marta, Stefano, Shuntaro and Valentijn, for the interesting talks in our LENT seminar and/or for the corona walks. En tenslotte, Jean Arthur, Ria Bekkering-Bosboom, Cécile Lemmette, Sylvia Visser en Linda Werensteijn, bedankt voor jullie goede zorgen vanuit het secretariaat.

Sander Dahmen en Peter Bruin, de zeer inspirerende MasterMath colleges over modulaire vormen hebben er zeker aan bijgedragen dat ik bij het onderwerp van mijn proefschrift ben gekomen. Marvin, thank you for your company during two conferences and inviting me to Frankfurt. Henrik, although we haven't yet proven your main conjecture, I really enjoy(ed) the many interesting conversations and results on our shared interests. You did not only introduce me to the fascinating world of multiple zeta values, but also organized a fantastic trip to Japan. Georg, it was a lot of fun to guess the pattern in the data you provided me with, and I learned a lot from you about quasi-Jacobi forms, Lagrange inversion and the usage of piecewise polynomials. Adrien, Danilo, Elba, Felix, Gabriele, Jiacheng, Jonas, Hanneke, Markus, Nils, Nobuo, Reinier, Yuta and many others, thank you for the time together at several conferences and/or interesting conversations. Finally, thank to Svenja Beljaars for arranging everything for my visits to the MPIM, and to Marianna Mäkelä for easing the communication with Don.

Dank aan broeder John en de andere broeders van Sint Jan voor de mooie gemeenschap waar ik acht jaar in heb gewoond, en voor het ter beschikking stellen van het Leerhuis voor het 'coronakantoor' van Jelmer, mij en anderen. Niko, erg bedankt voor de vele interessante colleges en goede adviezen. Vielen Dank an Norbert und das Althaus in Bonn für den herzlichen Empfang bei meinen Besuchen im MPIM.

Guido e Paola, grazie per l'accoglienza sempre calorosa. Chi l'avrebbe mai detto che avrei imparato una nuova lingua per divertimento, preparando la mia tesi di laurea? Papa, mama, Maaike en Clara, dank voor alles dat bijdraagt aan het mooie gezin dat wij vormen!

Maria Teresa, het is een prachtig avontuur om met jou de vreugde van de liefde te leven. Jouw geduld en liefdevolle vermaningen wanneer ik (te) druk was betekenen veel voor mij.

Benedícite, omnia opera Dómini, Dómino; laudáte et superexaltáte eum in saécula. 


\section{Curriculum Vitae}

Jan-Willem van Ittersum was born on August 10, 1994 in Amstelveen, the Netherlands. He started his bachelor studies in both Mathematics and Physics at Utrecht University in 2012. Under the supervision of Gunther Cornelissen he completed his Bachelor thesis titled Mahler's Measure and Möbius Transformations. He continued with a master in Mathematics, writing his thesis Two Problems Related to the Circle Method under supervision of Damaris Schindler. These theses both resulted in a publication.

In September 2017 he started his $\mathrm{PhD}$ project Examples, reformulations and generalisations of the Bloch-Okounkov theorem under supervision of both Gunther Cornelissen and Don Zagier. The main questions raised in his research proposal are answered in this thesis and resulted in three single-authored articles. During these years, he attended several international schools and conferences. These visits led to collaboration resulting in two further research articles partially included in this thesis.

Starting September 2021, he will be a Postdoctoral Fellow at the Max Planck Institute for Mathematics in Bonn. 



\section{Bibliography}

[ALS16] Alexander Alexandrov, Danilo Lewanski, and Sergey Shadrin. Ramifications of Hurwitz theory, KP integrability and quantum curves. J. High Energy Phys., 2016(5):124, 2016.

[AIK14] Tsuneo Arakawa, Tomoyoshi Ibukiyama, and Masanobu Kaneko. Bernoulli numbers and zeta functions. Springer Monographs in Mathematics. Springer, Tokyo, 2014. With an appendix by Don Zagier.

[ABR01] Sheldon Axler, Paul Bourdon, and Wade Ramey. Harmonic function theory, volume 137 of Graduate Texts in Mathematics. Springer-Verlag, New York, second edition, 2001.

[BO00] Spencer Bloch and Andrei Okounkov. The character of the infinite wedge representation. Adv. Math., 149(1):1-60, 2000.

[Boy 15] Hatice Boylan. Jacobi forms, finite quadratic modules and Weil representations over number fields, volume 2130 of Lecture Notes in Mathematics. Springer, Cham, 2015.

[Bri18] Kathrin Bringmann. Taylor coefficients of non-holomorphic Jacobi forms and applications. Res. Math. Sci., 5(1), 15:1-16, 2018.

[BM15] Kathrin Bringmann and Antun Milas. On the Fourier expansion of BlochOkounkov n-point function. J. Combin. Theory Ser. A, 136:201-219, 2015.

[BOW20] Kathrin Bringmann, Ken Ono, and Ian Wagner. Eichler integrals of Eisenstein series as $q$-brackets of weighted $t$-hook functions on partitions. ArXiv e-prints: 2009.07236, 13 pp., 2020.

[BKY18] Jim Bryan, Martijn Kool, and Benjamin Young. Trace identities for the topological vertex. Selecta Math. (N.S.), 24(2):1527-1548, 2018.

[CM16] Renzo Cavalieri and Eric Miles. Riemann surfaces and algebraic curves: A first course in Hurwitz theory, volume 87 of London Mathematical Society Student Texts. Cambridge University Press, Cambridge, 2016.

[CMSZ20] Dawei Chen, Martin Möller, Adrien Sauvaget, and Don Zagier. Masur-Veech volumes and intersection theory on moduli spaces of Abelian differentials. Invent. Math., 222(1):283-373, 2020. 
[CMZ18] Dawei Chen, Martin Möller, and Don Zagier. Quasimodularity and large genus limits of Siegel-Veech constants. J. Amer. Math. Soc., 31(4):1059-1163, 2018.

[Coh75] Henri Cohen. Sums involving the values at negative integers of $L$-functions of quadratic characters. Math. Ann., 217(3):271-285, 1975.

[CJ12] Gunther Cornelissen and Jan Willem de Jong. The spectral length of a map between Riemannian manifolds. J. Noncommut. Geom., 6(4):721-748, 2012.

[DMZ14] Atish Dabholkar, Sameer Murthy, and Don Zagier. Quantum black holes, wall crossing, and mock modular forms. ArXiv e-prints: 1208.4074, to appear in Cambridge Monogr. Math. Phys., 153 pp., 2014.

[Dan75] Jyotsna S. Dani. Density properties of orbits under discrete groups. J. Indian Math. Soc. (N.S.), 39:189-217 (1976), 1975.

[Dij95] Robbert Dijkgraaf. Mirror symmetry and elliptic curves. In The moduli space of curves (Texel Island, 1994), R. Dijkgraaf, C. Faber, and G. van der Geer, eds., volume 129 of Progr. Math., pp. 149-163. Birkhäuser Boston, Boston, MA, 1995.

[EZ85] Martin Eichler and Don Zagier. The theory of Jacobi forms, volume 55 of Progress in Mathematics. Birkhäuser Boston, Inc., Boston, MA, 1985.

[Eng17] Philip Engel. Hurwitz Theory of Elliptic Orbifolds, I. ArXiv e-prints: 1706.06738, to appear in Geom. Topol., 36 pp., 2017.

[EO01] Alex Eskin and Andrei Okounkov. Asymptotics of numbers of branched coverings of a torus and volumes of moduli spaces of holomorphic differentials. Invent. Math., 145(1):59-103, 2001.

[EO06] Alex Eskin and Andrei Okounkov. Pillowcases and quasimodular forms. In Algebraic geometry and number theory, volume 253 of Progr. Math., pp. 1-25. Birkhäuser Boston, Boston, MA, 2006.

[EOP08] Alex Eskin, Andrei Okounkov, and Rahul Pandharipande. The theta characteristic of a branched covering. Adv. Math., 217(3):873-888, 2008.

[GKZ06] Herbert Gangl, Masanobu Kaneko, and Don Zagier. Double zeta values and modular forms. In Automorphic forms and zeta functions, pages 71-106. World Sci. Publ., Hackensack, NJ, 2006.

[GM20] Elise Goujard and Martin Möller. Counting Feynman-like graphs: Quasimodularity and Siegel-Veech weight. J. Eur. Math. Soc. (JEMS), 22(2):365-412, 2020 .

[GJT16] Michael J. Griffin, Marie Jameson, and Sarah Trebat-Leder. On $p$-adic modular forms and the Bloch-Okounkov theorem. Res. Math. Sci., 3:11, 14 pp., 2016.

[Gui10] Antonin Guilloux. A brief remark on orbits of $\operatorname{SL}(2, \mathbb{Z})$ in the Euclidean plane. Ergodic Theory Dynam. Systems, 30(4):1101-1109, 2010. 
[HIL19] Marvin A. Hahn, Jan-Willem M. van Ittersum, and Felix Leid. Triply mixed coverings of arbitrary base curves: Quasimodularity, quantum curves and recursions. ArXiv e-prints:1901.03598, to appear in Ann. Henri Poincaré D, 41 pp., 2019.

[Hur1891] Adolf Hurwitz. Über Riemann'sche Flächen mit gegebenen Verzweigungspunkten. Math. Ann., 39(1):1-60, 1891.

[Hur1901] Adolf Hurwitz. Ueber die Anzahl der Riemann'schen Flächen mit gegebenen Verzweigungspunkten. Mathematische Annalen, 55(1):53-66, 1901.

[I20] Jan-Willem M. van Ittersum. When is the Bloch-Okounkov $q$-bracket modular? Ramanujan J., 52(3):669-682, 2020.

[I21a] Jan-Willem M. van Ittersum. A symmetric Bloch-Okounkov theorem. Res. Math. Sci., 8(19), 42 pp., 2021.

[I21b] Jan-Willem M. van Ittersum. The Bloch-Okounkov theorem for congruence subgroups and Taylor coefficients of quasi-Jacobi forms. ArXiv e-prints: 2102.12964, 49 pp., 2021.

[IOP20] Jan-Willem van Ittersum, Georg Oberdieck, and Aaron Pixton. Gromov-Witten theory of K3 surfaces and a Kaneko-Zagier equation for Jacobi forms. ArXiv e-prints: 2007.03489, 30 pp., 2020.

[Juc74] Algimantas A.A. Jucys. Symmetric polynomials and the center of the symmetric group ring. Reports on Mathematical Physics, 5(1):107-112, 1974.

[KK03] Masanobu Kaneko and Masao Koike. On modular forms arising from a differential equation of hypergeometric type. Ramanujan J., 7:145-164, 2003.

[KK04] Masanobu Kaneko and Masao Koike. Quasimodular solutions of a differential equation of hypergeometric type. In Galois theory and modular forms, K. Hashimoto, K. Miyake, and H. Nakamura, eds., volume 11 of Dev. Math., pp. 329-336. Kluwer Acad. Publ., Boston, MA, 2004.

[KZ95] Masanobu Kaneko and Don Zagier. A generalized Jacobi theta function and quasimodular forms. In The moduli space of curves (Texel Island, 1994), R. Dijkgraaf, C. Faber, and G. van der Geer, eds., volume 129 of Progr. Math., pp. 149-163. Birkhäuser Boston, Boston, MA, 1995.

[KZ98] Masanobu Kaneko and Don Zagier. Supersingular $j$-invariants, hypergeometric series, and Atkin's orthogonal polynomials. In Computational perspectives on number theory (Chicago, IL, 1995), volume 7 of AMS/IP Stud. Adv. Math., pages 97-126. Amer. Math. Soc., Providence, RI, 1998.

[KO94] Serguei Kerov and Grigori Olshanski. Polynomial functions on the set of Young diagrams. C. R. Acad. Sci., Paris, Sér. I, 319(2):121-126, 1994.

[Kiy16] Tomoya Kiyuna. Kaneko-Zagier type equation for Jacobi forms of index 1. Ramanujan J., 39(2):347-362, 2016. 
[Kro1884] Leopold Kronecker. Näherungsweise ganzzahlige Auflösung linearer Gleichungen. Monatsber. Königl. Preuß. Akad. Wiss. Berlin, 1179-1193 and 1271-1299 (1884)

[KL81] Daniel S. Kubert and Serge Lang. Modular units, volume 244 of Grundlehren der Mathematischen Wissenschaften [Fundamental Principles of Mathematical Science]. Springer-Verlag, New York-Berlin, 1981.

[LN12] Michel Laurent and Arnaldo Nogueira. Approximation to points in the plane by SL(2, Z)-orbits. J. Lond. Math. Soc. (2), 85(2):409-429, 2012.

[Lib11] Anatoly Libgober. Elliptic genera, real algebraic varieties and quasi-Jacobi forms. In Topology of stratified spaces, volume 58 of Math. Sci. Res. Inst. Publ., pp. 95-120. Cambridge Univ. Press, Cambridge, 2011.

[LD20] David Lowry-Duda. Visualizing modular forms. ArXiv e-prints: 2002.05234, 20 pp., 2020.

[Mur81] G. Eugene Murphy. A new construction of Young's seminormal representation of the symmetric groups. Journal of Algebra, 69(2):287-297, 1981.

[Nak19] 中屋智瑛 (Tomoaki Nakaya). 古典的楕円関数と保型微分方程式につ いて [On classical elliptic functions and automorphic differential equations]. Unpublished conference proceedings of the 13th Fukuoka mathematical research conference, communicated by Masanobu Kaneko.

[NO06] Nikita A. Nekrasov and Andrei Okounkov. Seiberg-Witten theory and random partitions. In The unity of mathematics, P. Etingof, V. Retakh, I. M. Singer, eds., volume 244 of Progr. Math., pp. 525-596. Birkhäuser Boston, Boston, MA, 2006.

[Obe18] Georg Oberdieck. Gromov-Witten invariants of the Hilbert schemes of points of a K3 surface. Geom. Topol., 22(1):323-437, 2018.

[OP16] Georg Oberdieck and Rahul Pandharipande. Curve counting on $K 3 \times E$, the Igusa cusp form $\chi_{10}$, and descendent integration. In K3 surfaces and their moduli, volume 315 of Progr. Math., pp. 245-278. Birkhäuser/Springer, [Cham], 2016.

[OP18] Georg Oberdieck and Aaron Pixton. Holomorphic anomaly equations and the Igusa cusp form conjecture. Invent. Math., 213(2):507-587, 2018.

[OP19] Georg Oberdieck and Aaron Pixton. Gromov-Witten theory of elliptic fibrations: Jacobi forms and holomorphic anomaly equations. Geom. Topol., 23(3):1415-1489, 2019.

[Och01] 落合啓之 (Hiroyuki Ochiai). Counting functions for branched covers of elliptic curves and quasi-modular forms. In Representation theory of vertex operator algebras and related topics (Japanese) (Kyoto, 2000), pp. 153-167. 2001.

[O097] Andrei Okounkov and Grigori Olshanski. Shifted Schur functions. Algebra $i$ Analiz, 9(2):73-146, 1997. 
[OP06] Andrei Okounkov and Rahul Pandharipande. Gromov-Witten theory, Hurwitz theory, and completed cycles. Ann. of Math. (2), 163:517-560, 2006.

[Ran56] Robert A. Rankin. The construction of automorphic forms from the derivatives of a given form. J. Indian Math. Soc. (N.S.), 20:103-116, 1956.

[Rot64] Gian-Carlo Rota. On the foundations of combinatorial theory. I. Theory of Möbius functions. Z. Wahrscheinlichkeitstheorie und Verw. Gebiete, 2:340-368 (1964), 1964.

[RY10] Mike Roth and Noriko Yui. Mirror symmetry for elliptic curves: the A-model (fermionic) counting. In Motives, quantum field theory, and pseudodifferential operators, A. Carey, D. Ellwood, S. Paycha, S. Rosenberg, eds., volume 12 of Clay Math. Proc., pp. 245-283. Amer. Math. Soc., Providence, RI, 2010.

[Sage17] The Sage Developers. SageMath, the Sage Mathematics Software System (Version 8.0), 2017. http: //www . sagemath .org.

[Sch17] Robert Schneider. Arithmetic of partitions and the $q$-bracket operator. Proc. Amer. Math. Soc., 145(5):1953-1968, 2017.

[Sch39] Bruno Schoeneberg. Das Verhalten von mehrfachen Thetareihen bei Modulsubstitutionen. Math. Ann., 116(1):511-523, 1939.

[Wei99] André Weil. Elliptic functions according to Eisenstein and Kronecker. Classics in Mathematics. Springer-Verlag, Berlin, 1999. Reprint of the 1976 original.

[Zag77] Don Zagier. Modular forms whose Fourier coefficients involve zeta-functions of quadratic fields. In Modular functions of one variable, VI (Proc. Second Internat. Conf., Univ. Bonn, Bonn, 1976), J.-P. Serre, D. B. Zagier, eds., volume 627 of Lecture Notes in Math., pp. 105-169. Springer, Berlin, 1977.

[Zag91] Don Zagier. Periods of modular forms and Jacobi theta functions. Invent. Math., 104(3):449-465, 1991.

[Zag94] Don Zagier. Modular forms and differential operators. Proc. Indian Acad. Sci. Math. Sci., 104:57-75. 1994.

[Zag08] Don Zagier. Elliptic modular forms and their applications. In The 1-2-3 of modular forms, K. Ranestad, ed., Universitext, pp. 1-103. Springer, Berlin, 2008.

[Zag16] Don Zagier. Partitions, quasimodular forms, and the Bloch-Okounkov theorem. Ramanujan J., 41(1-3):345-368, 2016.

[Zwe02] Sander P. Zwegers. Mock Theta Functions. PhD thesis, Universiteit Utrecht, 87 pp., 2002, https://dspace. library . uu.nl/handle/1874/878. 


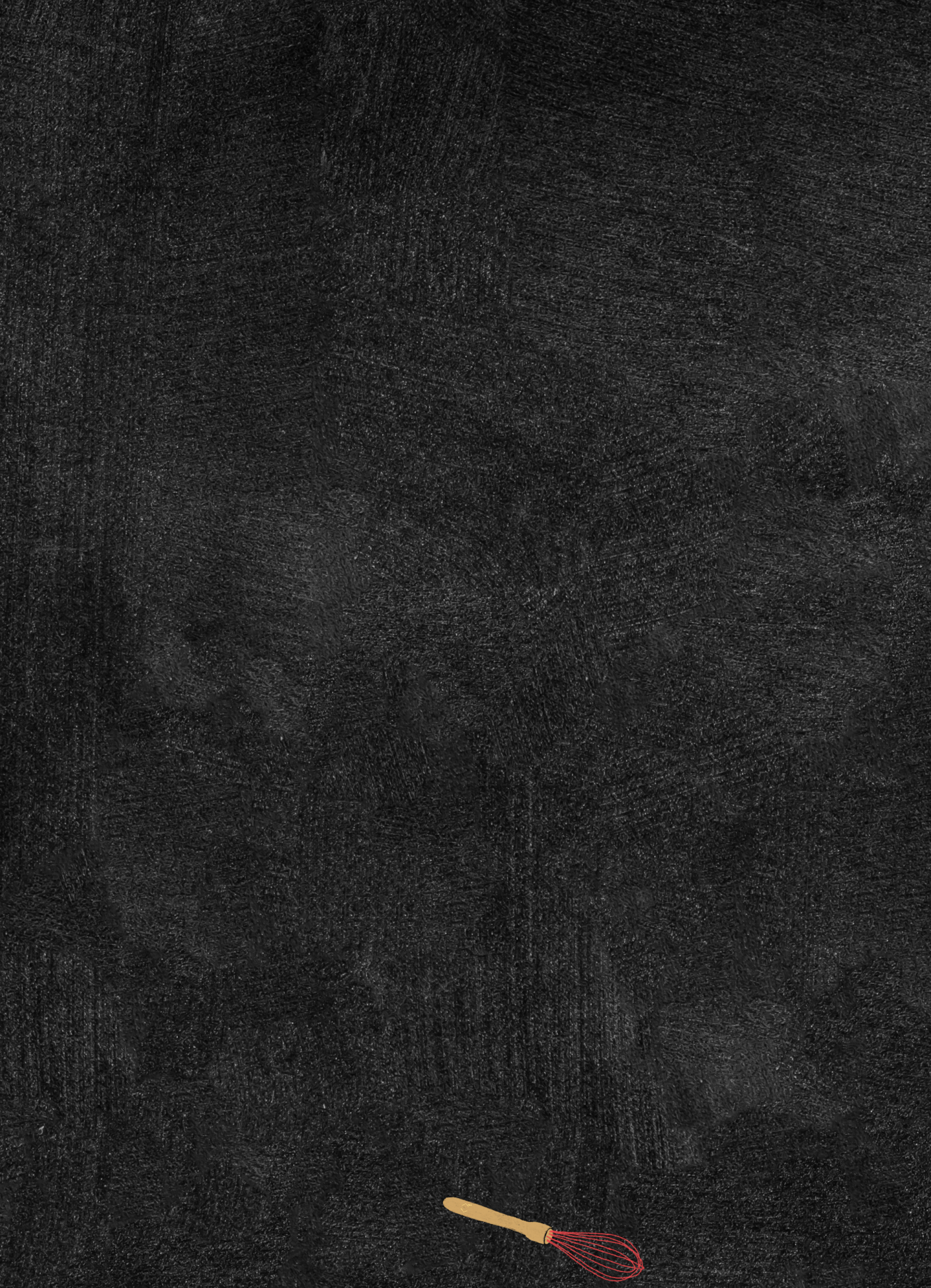

